\title{
X-ray waveguides and waveguide-based lensless imaging
}

\author{
Dissertation \\ zur Erlangung des Doktorgrades \\ der Mathematisch-Naturwissenschaftlichen Fakultäten \\ der Georg-August-Universität zu Göttingen
}

vorgelegt von

Christian Fuhse

aus Osterode am Harz

Göttingen 2006 
D7

Referent: Prof. Dr. Tim Salditt

Korreferent: Prof. Dr. Hans-Ulrich Krebs

Tag der mündlichen Prüfung: 9. Mai 2006 


\section{Contents}

\begin{tabular}{lll}
\hline & Introduction & 1
\end{tabular}

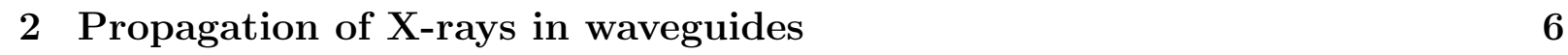

2.1 Refraction and total reflection of X-rays . . . . . . . . . . . . . . . 6

2.2 Propagation of X-rays in matter . . . . . . . . . . . . . . . . . . . . 7

2.3 One-dimensionally confining waveguides $\ldots \ldots \ldots$. . . . . . . . . . . 8

2.3 .1 The symmetric slab waveguide . . . . . . . . . . . . . . . . 9

2.3 .2 Direct coupling . . . . . . . . . . . . . . . . . . 11

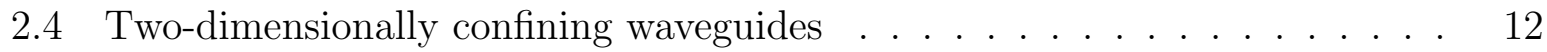

2.4 .1 The weakly-guiding fibre . . . . . . . . . . . . . . . . . 12

2.5 The Fraunhofer diffraction pattern . . . . . . . . . . . . . . . . . . . . . 14

2.6 The parabolic wave equation $\ldots \ldots \ldots \ldots \ldots$

2.7 The limits of confinement . . . . . . . . . . . . . . . . . . . . . . 15

2.7 .1 Comparison to the limits of other focusing optics . . . . . . . . 17

2.8 Dimensionless coordinates $\ldots \ldots \ldots \ldots$

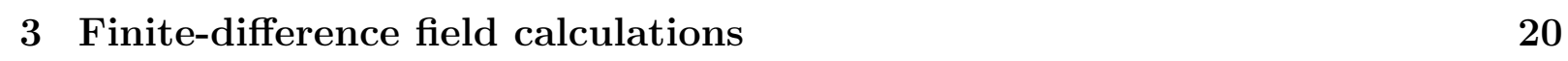

3.1 Discretisation and boundary conditions . . . . . . . . . . . . . . . . . . 20

3.2 One-dimensionally confining waveguides . . . . . . . . . . . . . . 22

3.2 .1 Comparison to analytical results . . . . . . . . . . . . . . . . . 23

3.3 Two-dimensionally confining waveguides $\ldots \ldots \ldots$. . . . . . . . . . 27

3.3 .1 Comparison to the weakly-guiding fibre . . . . . . . . . . . . . . 30

3.4 Conclusions . . . . . . . . . . . . . . . . . . . . . . . . . . . . . . . . . . . . 32

4 Propagation of X-rays in ultra-narrow apertures 33

4.1 Onset of waveguiding effects in small apertures . . . . . . . . . . . . . . . . 33

4.2 Transmission of single-mode waveguides $\ldots \ldots \ldots$. . . . . . . . . . . . . . 39

4.3 Conclusions . . . . . . . . . . . . . . . . . . . . . . . . . . . . . . . . . 43

$\begin{array}{lll}5 & \text { Waveguide-based imaging } & 44\end{array}$

5.1 Scalar diffraction theory . . . . . . . . . . . . . . . . . . . 45

5.1 .1 The optical transmission function . . . . . . . . . . . . . 47

5.1 .2 Contrast transfer function for weak objects . . . . . . . . . . . . . . 47

$5.1 .3 \quad$ Validity of the Fresnel approximation . . . . . . . . . . . . . . . 49

5.2 Holography . . . . . . . . . . . . . . . . . . . 50

$5.2 .1 \quad$ In-line holography with a plane wave . . . . . . . . . . . . . 51 
5.2 .2 In-line holography with a point source . . . . . . . . . . . 52

5.2 .3 Off-axis holography . . . . . . . . . . . . . . . . . . 53

5.2 .4 Waveguide-based holography . . . . . . . . . . . . . . . 54

5.3 Practical considerations . . . . . . . . . . . . . . . . 57

5.3 .1 Imaging regimes in waveguide-based imaging . . . . . . . . . . . . 57

5.3 .2 Noise . . . . . . . . . . . . . . . . . . . . 60

5.4 Conclusions . . . . . . . . . . . . . . . . . . . . . . . 62

6 Experiments I: Characterisation of X-ray waveguides 63

6.1 Waveguide design . . . . . . . . . . . . . . . . . 63

6.2 Fabrication of X-ray waveguides . . . . . . . . . . . . . . . . 64

6.3 Coupling of X-rays into waveguides . . . . . . . . . . . . . . . . 66

$6.4 \quad$ Direct coupling into a single-mode planar waveguide . . . . . . . . . . . . . 67

6.4 .1 Experiment . . . . . . . . . . . . . . . . . . 67

6.4 .2 Results . . . . . . . . . . . . . . . . . . . . . . . . . . . . 69

6.4 .3 Conclusions . . . . . . . . . . . . . . . . 70

6.5 Coherent propagation of white X-rays in a planar waveguide . . . . . . . 70

6.5 .1 Experiment . . . . . . . . . . . . . . . . . 72

6.5 .2 Results . . . . . . . . . . . . . . . . . . . 73

6.5 .3 Conclusions . . . . . . . . . . . . . . . . . . . 76

6.6 Direct coupling into two-dimensionally confining waveguides . . . . . . . . 76

6.6 .1 Experiment . . . . . . . . . . . . . . . . 76

6.6 .2 Results . . . . . . . . . . . . . . . . . . . 76

6.6 .3 Conclusions . . . . . . . . . . . . . . . . . . . . . . . . 79

6.7 Waveguide Gratings $\ldots \ldots \ldots$. . . . . . . . . . . . . . . . . . 79

6.7 .1 Experiment . . . . . . . . . . . . . . . . 80

6.7 .2 Results . . . . . . . . . . . . . . . . . . . 80

6.7 .3 Conclusions . . . . . . . . . . . . . . . . . . . . . . . . . . 81

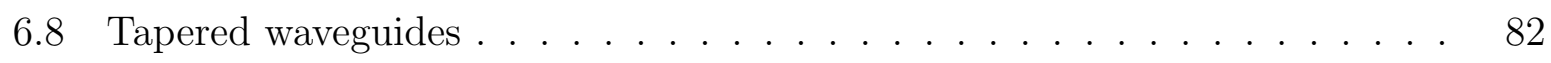

6.8 .1 Experiment . . . . . . . . . . . . . . . . 82

6.8 .2 Results . . . . . . . . . . . . . . . . . . . . . . 84

6.8 .3 Conclusions . . . . . . . . . . . . . . . . . . . . . . . 84

6.9 Curved waveguides and double waveguides . . . . . . . . . . . . . . 85

$6.9 .1 \quad$ Double waveguides . . . . . . . . . . . . . . . . 85

6.9 .2 Curved waveguides . . . . . . . . . . . . . . . . 88

6.9 .3 Y double waveguides . . . . . . . . . . . . . . . . . . . . . . . 90

6.9 .4 Conclusions . . . . . . . . . . . . . . . . . . . . . . 91

6.10 Conclusions . . . . . . . . . . . . . . . . . . . . . . . . 93 
\begin{tabular}{|lll}
7 & Experiments II: Waveguide-based imaging & 95
\end{tabular}

7.1 Brilliance . . . . . . . . . . . . . . . . . . . . . . . . . . . 95

7.2 Experiment setup $\ldots \ldots \ldots \ldots$

7.3 Preparation of test samples $\ldots \ldots \ldots \ldots \ldots$

7.4 Direct imaging $\ldots \ldots \ldots \ldots y$

$7.5 \quad$ Holographic imaging $\ldots \ldots \ldots \ldots$

7.6 Imaging of $\mathrm{Cu}$ islands $\ldots \ldots \ldots \ldots$

7.7 Off-axis holography $\ldots \ldots \ldots \ldots \ldots$

7.8 Conclusions . . . . . . . . . . . . . . . . . . . . . . . . . . . . . . . . . . 109

$\begin{array}{lll}8 & \text { Summary } & 111\end{array}$

\begin{tabular}{ll}
\hline Bibliography & 115
\end{tabular}

\begin{tabular}{ll}
\hline A Appendix & 125
\end{tabular}

A.1 The Fourier transform . . . . . . . . . . . . . . . . . . . . . . 125

A.2 Analogy between parallel-beam and point-source holography . . . . . . . 126

A.2.1 In-line holography . . . . . . . . . . . . . . . . . . 126

A.2.2 Off-axis holography . . . . . . . . . . . . . . . . . . . . . . . . . 128

A.3 Equivalence of the paraxial approximation of the Fresnel-Kirchhoff integral

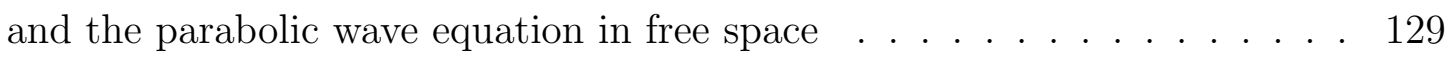

A.4 IDL source code . . . . . . . . . . . . . . . . . . . . . . . . . . . . 130

A.4.1 Finite-difference calculations in two dimensions . . . . . . . . 130

A.4.2 Finite-difference calculations in three dimensions. . . . . . . . . . . 133

A.4.3 A propagation algorithm for holographic reconstruction . . . . . . . 138

A.4.4 Off-axis holographic reconstruction . . . . . . . . . . . . . . . 139

\begin{tabular}{ll}
\hline B List of publications & 143
\end{tabular}

\begin{tabular}{ll}
\hline C Acknowledgements & 145
\end{tabular} 


\section{Introduction}

Microscopy is carried out with a variety of probes. Techniques like visible-light microscopy, electron microscopy, scanning tunnelling microscopy or scanning force microscopy are highly developed and routinely used. Each technique has its own merits and limitations, in particular regarding spatial resolution, and is sensitive to a specific physical quantity. Therefore, the choice of the best-suited technique depends on the specific specimen and on the physical quantity to be investigated.

With the availability of highly brilliant synchrotron radiation and with rapid advances in fabrication of X-ray optical devices, X-ray microscopy has developed remarkably over the last few decades [KJH95, SJP03, SS06]. Due to smaller wavelengths, X-ray microscopy has the potential for a significantly higher spatial resolution than visible-light microscopy. In contrast to electron microscopy, the sample does not need to be put into a vacuum. This allows for instance the studying of biological specimen in water or the studying of samples embedded in a solid. X-rays penetrate comparably thick samples and thus allow a deep look inside a sample rather than only at its surface. Moreover, X-ray microscopy is an inherently non-destructive technique and no invasive sample preparation is necessary.

Without claim of completeness, we divide the available X-ray microscopy techniques into different categories, as illustrated in Fig. 1.1. Basically, we distinguish between fullfield and scanning techniques. The latter are based on scanning a focused beam across the sample while measuring local X-ray absorption, fluorescence, diffraction or differential phase contrast.

Full-field techniques may be further divided into lens-based transmission microscopy and lensless techniques, namely image reconstruction from coherent diffraction data and lensless projection imaging. Lens-based transmission microscopes [GNR ${ }^{+} 03$ ] are constructed similarly to conventional visible-light microscopes. But a major problem in X-ray microscopy is comparably poor lenses. A simple and efficient refractive lens comparable to lenses for visible light is not available, since the refractive index $n=1-\delta-i b$ of all real materials is close to 1 and absorption is crucial. Fresnel-zone-plate lenses are used in particular for soft X-rays [ $\left.\mathrm{CHL}^{+} 05\right]$, and arrays of many individual refractive lenses ("compound refractive lenses") are used in the hard X-ray regime $(\lambda \lesssim 0.1 \mathrm{~nm}$ ) where absorption is lower $\left[\mathrm{SKP}^{+} 05\right]$. However, fabrication of these lenses is difficult and spatial resolution is still far from the wavelength limit.

Motivated by the difficulties of lens fabrication, various lensless full-field imaging techniques have been developed. Highest spatial resolution has been achieved by image reconstruction from coherent diffraction data [MCKS99, MIAH03]. The Fraunhofer (far-field) diffraction pattern of a coherently illuminated sample corresponds to the Fourier transform 


\section{X-ray microscopy}

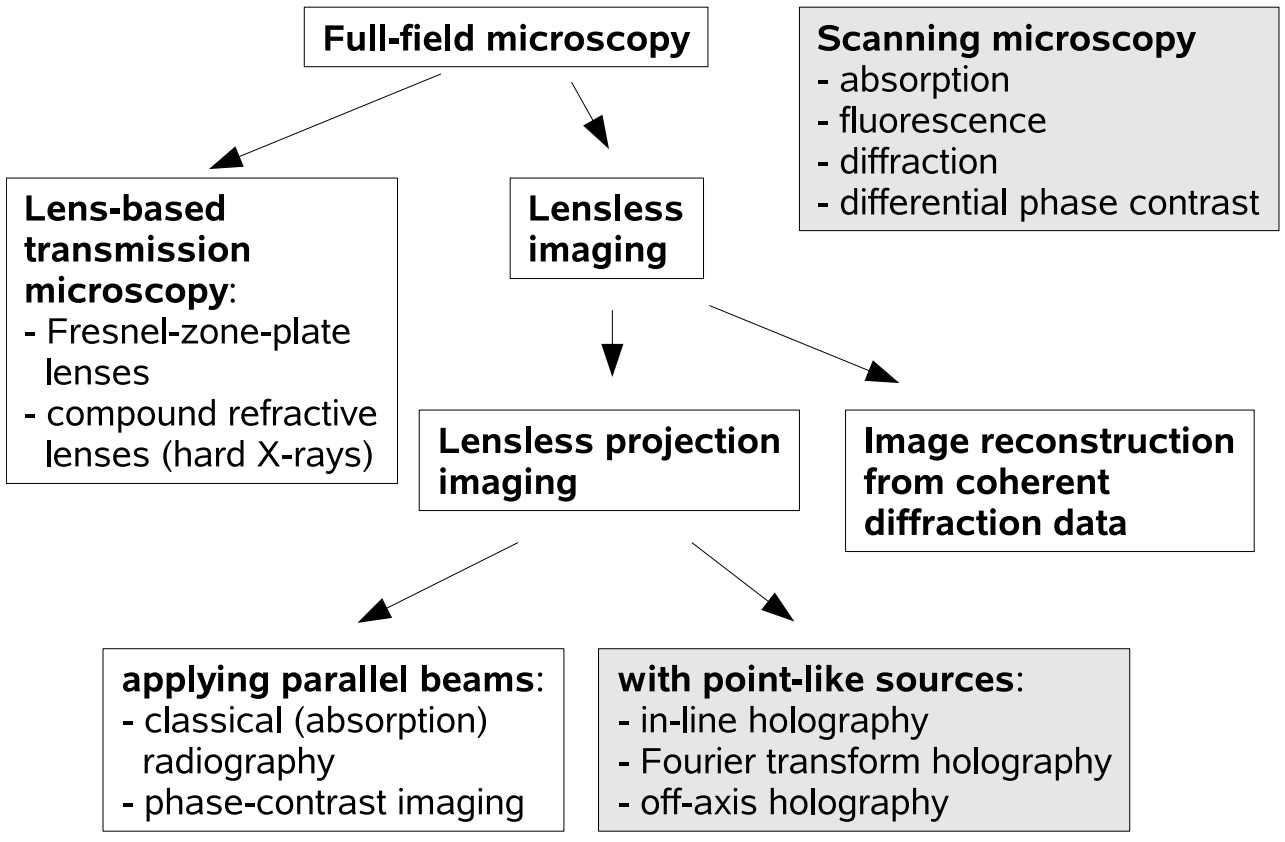

Figure 1.1: Overview of various X-ray microscopy techniques. Spatial resolution of the techniques indicated by grey-shaded boxes depends on the spatial extent of a small probe.

of the electron density. The latter may in principle be recovered from the diffraction pattern by Fourier back-transformation. However, phase information is lost when detecting the scattering signal and has to be recovered using iterative algorithms and oversampling [Fie78]. Besides technical difficulties, convergence and uniqueness of the reconstruction are severe problems.

In parallel, lensless projection microscopy has been developed [SSKS95]. High spatial resolution is obtained by either using a parallel beam and a high-resolution detector, or by applying a divergent beam and recording a magnified image. In the latter case, spatial resolution is determined by the spatial extent of the source. As shown in this work, the magnified image of a small object is not simply given by a magnified absorption image as known from classical X-ray radiography, but rather by a diffraction pattern. Furthermore, with increasing photon energy and decreasing sample size, absorption decreases and phase contrast becomes important. The phase shift originating from a sample is directly related to the local electron density and the latter may in principle be deduced from the detected diffraction pattern. Again, the aim is to reconstruct the X-ray wave from a detected intensity pattern. This scheme is well-known from visible light as holography, and in-line holography, off-axis holography or Fourier transform holography can be extended to the $\mathrm{X}$-ray regime [HJK $\left.{ }^{+} 87, \mathrm{MKJ}^{+} 92, \mathrm{KS}^{+} 04\right]$. 


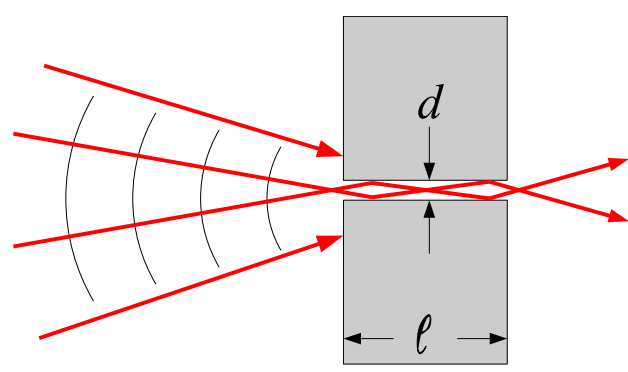

Figure 1.2: Simple ray-optical picture of a small aperture with diameter $d$ placed in the focal spot of an X-ray focusing device to further decrease the spot size. Due to the large penetration depth, the aperture has to be surrounded by material of a comparably large thickness $\ell$. The aperture therefore becomes a long narrow channel with a high aspect ratio $\ell / d$. Accordingly, $X$-rays transmitted through the channel propagate at very small grazing angles and reflection at the interfaces leads to waveguiding effects.

As indicated in Fig. 1.1, spatial resolution of many techniques depends on the size of a "point-like" source, usually the focal spot of a focusing device. However, focal spot sizes of the present X-ray focusing devices are still far away from the wavelength limit. As illustrated in Fig. 1.2, one may consider further decreasing the spot size with an extremely small aperture. Due to the large penetration depth in particular for hard X-rays, the aperture has to be surrounded by material with a thickness in the order of micrometres or even millimetres. An aperture with cross-section dimensions of $100 \mathrm{~nm}$ or below then corresponds to a narrow channel rather than to a hole in a flat opaque screen. X-rays are only transmitted through the channel when propagating at very small grazing angles. At these small angles, X-rays are reflected from the interfaces of the channel and the beam becomes "guided". In a more elaborate wave-optical consideration, the channel transpires to be a waveguide and the incident beam excites guided modes.

$\mathrm{X}$-ray waveguides indeed allow the confinement of X-rays in one or two dimensions in a guiding core with cross-sectional dimensions of some tens of nanometres. This is significantly below the focal spot sizes of hard-X-ray focusing devices in particular. The beam exiting a waveguide is highly coherent and has a typical divergence of a few milliradians.

One-dimensional X-ray beam confinement in a planar waveguide was demonstrated by Spiller and Segmüller in 1973 [SS73]. In 1995, Feng et al. measured the Fraunhofer diffraction pattern originating from the end face of a planar waveguide [ $\left.\mathrm{FSF}^{+} 95\right]$. They found a fully coherent beam and measured diffraction patterns related to individual guided modes. Proof-of-principle experiments for applications of waveguides as optical elements in X-ray microscopy have been carried out demonstrating the feasibility of lensless phase-contrast projection imaging [ $\left.\mathrm{LCC}^{+} 97\right]$ and scanning X-ray diffraction microscopy $\left[\mathrm{JLG}^{+} 00, \mathrm{CLS}^{+} 04\right]$. One-dimensional confinement in planar waveguides provides high spatial resolution in one direction, and the experiments allowed the studying of appropriate samples which do not require high resolution in two directions, like thin-film structures $\left[\mathrm{JLG}^{+} 00, \mathrm{CLS}^{+} 04\right]$ or fibres $\left.\mathrm{DBR}^{2}, \mathrm{LPB}^{+} 05\right]$. 
Two-dimensional X-ray beam compression was demonstrated using tapered glass capillaries with diameters of down to $\approx 100 \mathrm{~nm}$ [BHT94]. In 2002, Pfeiffer et al. presented a two-dimensionally confining $\mathrm{X}$-ray waveguide $\left[\mathrm{PDB}^{+} 02\right]$. The beam was coupled into the waveguide applying the so-called "resonant beam coupler" scheme. Guided modes are excited via an evanescent wave through a thin cladding. Although intensity of the beam was comparably low and the waveguide beam was accompanied by intense disturbing contributions of the primary and specularly reflected beams, the experiment has shown that two-dimensional confinement in X-ray waveguides is indeed feasible.

Based on these works, the aim of the present thesis is (i) to contribute to the understanding of wave propagation in X-ray waveguides, (ii) to design, fabricate, and characterise in particular two-dimensionally confining X-ray waveguides providing beams well-suited for microscopy applications, (iii) to develop a theoretical background for waveguide-based lensless full-field imaging, and (iv) to experimentally verify the feasibility of this technique.

The thesis is organised as follows: Chapter 2 describes the propagation of X-rays in matter, in particular in waveguides, by analytical theory. We consider analytical results for planar and cylindrical waveguides. Existing concepts are extended to account for absorption which is a critical parameter for X-ray waveguides. Since exact analytical solutions are rare, the parabolic wave equation is presented as a basis for numerical approximations. We then introduce dimensionless coordinates enabling more general conclusions.

Chapter 3 presents finite-difference algorithms solving the parabolic wave equation in two or three dimensions. We thus get a computational tool allowing us to calculate the electric field inside X-ray waveguides of practically arbitrary geometry. From the electric field we may deduce all relevant properties of the waveguide, like transmission, beam size, or coupling between waveguides. We may thus easily design appropriate waveguides for specific applications and experimental conditions.

In Chapter 4 we consider the propagation of X-rays in ultra-narrow slits and address the question of how small an aperture can get until it has to be considered a waveguide.

Chapter 5 gives the theoretical background for waveguide-based lensless imaging by means of scalar diffraction theory. We point out different imaging regimes and evaluate contrast and resolution. It transpires that high spatial resolution in the order of the crosssectional dimensions of the guiding core is achieved in the holographic regime. Therefore, we introduce waveguide-based in-line and off-axis holography.

Chapter 6 presents the fabrication and experimental characterisation of one-dimensionally and two-dimensionally confining X-ray waveguides. The experiments verify the theory of wave propagation in waveguides, and we demonstrate how sufficiently intense and undisturbed beams are obtained from direct coupling of a pre-focused undulator beam into the front face of the waveguide. We furthermore demonstrate how two coherent waveguide beams can be provided for off-axis holography. 
Chapter 7 presents the first imaging experiments based on two-dimensionally confining waveguides. Appropriate test samples are prepared for the different imaging regimes and we evaluate the respective image quality.

Chapter 8 finally summarises the results and presents the conclusions. 


\section{Propagation of X-rays in waveguides}

This chapter introduces the basic physical principles underlying the propagation of X-rays in waveguides. We consider the refractive index for electromagnetic radiation in the $X$-ray regime (Section 2.1) and describe the propagation of X-rays in matter by means of scalar wave theory (Section 2.2). We then discuss propagation of $X$-rays in one-dimensionally and two-dimensionally confining waveguides. Therefore, the guided modes of symmetric slab waveguides (Section 2.3) and cylinder waveguides (Section 2.4) are studied by analytical theory. Although the electric field inside an X-ray waveguide cannot be easily measured directly, it may be deduced from the far-field diffraction pattern (Section 2.5).

Since exact analytical solutions are rare, Section 2.6 introduces the parabolic wave equation as a basis for finite-difference field calculations, presented in Chapter 3. The parabolic wave equation furthermore points out a fundamental lower limit to which $X$ rays may be confined in waveguides (Section 2.7). Finally, we introduce dimensionless coordinates enabling more general conclusions (Section 2.8).

\subsection{Refraction and total reflection of X-rays}

The interaction of X-rays with matter is usually discussed at the atomic level where X-ray scattering and diffraction are well-known from physics textbooks Vog97. Since X-rays are electromagnetic waves, one may also expect refraction phenomena when X-rays penetrate a sharp interface between two different homogeneous media. The grazing angle $\theta_{i}$ of an incident beam propagating in vacuum and the grazing angle $\theta_{r}$ of the refracted beam are then related by Snell's law (see Fig. 2.1):

$$
\cos \theta_{i}=n \cos \theta_{r}
$$

The quantity $n$ is known as the refractive index of the medium. For X-rays, it transpires that $n$ is very close to 1 and accordingly it is usually written as

$$
n=1-\delta-i b
$$

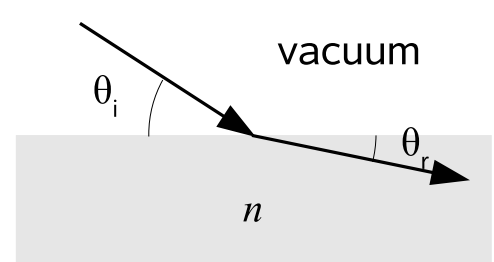

Figure 2.1: Refraction of an X-ray beam entering a homogeneous medium from vacuum. $\theta_{i}$ and $\theta_{r}$ denote the grazing angles of the incident and refracted beam, respectively. 
with $\delta$ and $b$ denoting the real and imaginary part of the decrement. Ignoring small dispersion corrections in the vicinity of absorption edges, the decrement $\delta$ is given by ANM01

$$
\delta=\frac{\lambda^{2} \varrho_{e} r_{e}}{2 \pi}
$$

where $r_{e} \approx 2.818 \times 10^{-15} \mathrm{~m}$ denotes the classical radius of the electron, $\varrho_{e}$ denotes the electron density, and $\lambda$ is the X-ray wavelength. Typically, $\delta$ is in the order of $10^{-6}$ for hard X-rays. The imaginary part $b$ is related to the linear attenuation coefficient $\mu$ by

$$
b=\frac{\mu \lambda}{4 \pi}
$$

and is typically smaller than $\delta$. Since the real-part of the refractive index is less than unity, $\mathrm{X}$-rays propagating in vacuum are totally reflected on the surface of any real material if the grazing angle is below a certain critical angle depending on the decrement $\delta$. An expansion of the cosine in Eq. (2.1) for $\theta_{i}=\theta_{c}$ and $\theta_{r}=0$ gives the critical angle of total external reflection:

$$
\theta_{c} \approx \sqrt{2 \delta}
$$

With $\delta$ in the order of $10^{-6}$ we find that the critical angle is in the order of milliradians. However, total reflection is the basis for guiding electromagnetic radiation in waveguides.

\subsection{Propagation of $\mathrm{X}$-rays in matter}

The propagation of electromagnetic waves in matter is described by the Maxwell equations [Vog97. In an uncharged, non-conducting and non-magnetic medium, the Maxwell equations read

$$
\begin{aligned}
\operatorname{curl} \boldsymbol{H} & =\frac{\partial \boldsymbol{D}}{\partial t}, \\
\operatorname{curl} \boldsymbol{E} & =-\frac{\partial \boldsymbol{B}}{\partial t}, \\
\operatorname{div} \boldsymbol{D} & =0, \\
\operatorname{div} \boldsymbol{B} & =0 .
\end{aligned}
$$

$\boldsymbol{E}$ denotes the electric field, $\boldsymbol{H}$ is the magnetic field intensity, $\boldsymbol{B}=\mu_{0} \boldsymbol{H}$ is the magnetic flux density, $\boldsymbol{D}=\epsilon \epsilon_{0} \boldsymbol{E}$ is the electric displacement field, and $\mu_{0}=4 \pi \times 10^{-7} \mathrm{VsA}^{-1} \mathrm{~m}^{-1}$ and $\epsilon_{0}=8.854 \times 10^{-12} \mathrm{AsV}^{-1} \mathrm{~m}^{-1}$ denote the permeability and permittivity of empty space. $\epsilon$ denotes the permittivity of the medium and is related to the refractive index by $\epsilon=n^{2}$. For monochromatic waves with time dependency given by a function $\exp (i \omega t)$, the Maxwell equations may be transformed into a wave equation given for the electric field by [LK03]:

$$
\nabla^{2} \boldsymbol{E}-\frac{1}{v^{2}} \frac{\partial^{2} \boldsymbol{E}}{\partial t^{2}}=0
$$




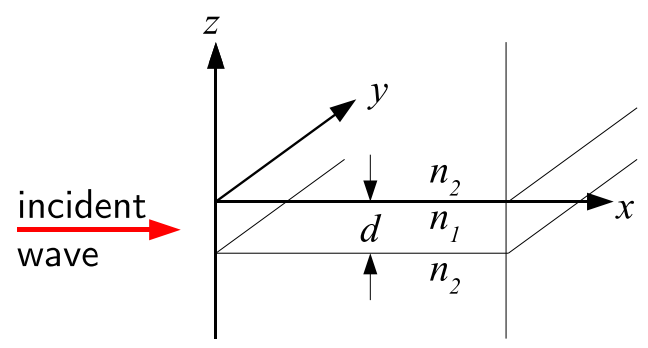

Figure 2.2: Schematic of a planar waveguide. The X-rays are confined in a layer with a refractive index $n_{1}$ which is higher than the refractive index $n_{2}$ in the cladding. The axes of coordinates are chosen in a way that the $x$ axis denotes the propagation direction and the $z$ axis is perpendicular to the guiding layer.

$v=c / n$ denotes the propagation speed with $c$, the speed of light in empty space. The experiments presented in this thesis are carried out using synchrotron radiation that is usually linearly polarised in a horizontal direction* Accordingly, we consider only the horizontal component of the electric field denoted by the symbol $\psi$. The propagation of monochromatic X-rays is then described by the Helmholtz equation BW87]

$$
\nabla^{2} \psi+k^{2} n^{2}(x, y, z) \psi=0
$$

where $k=\omega / c=2 \pi / \lambda$ is the wave number in free space and the refractive index is a function of the coordinates $(x, y, z)$.

\subsection{One-dimensionally confining waveguides}

Let us now consider the confinement of X-rays propagating in $x$ direction in a guiding core with diameter $d$, where the refractive index $n_{1}$ is larger than in the refractive index $n_{2}$ in the cladding (see Fig. 2.2). When an X-ray wave impinges on the front face of a waveguide, it is split into one component propagating mainly in the guiding core (guided modes [Mar74]) and one component propagating mainly in the cladding (radiative modes [Mar74]). According to Eq. (2.3), the smaller refractive index of the cladding corresponds to a higher electron density, i. e. typically to heavy elements with strong absorption. Thus, radiation propagating in the cladding is quickly damped out and the electric field at the end face of the waveguide is in practice generated by the contributions of guided modes.

This section is restricted to one-dimensional confinement, i. e. we assume that the electric field and the refractive index profile are independent of $y$. The scalar wave equation then reads

$$
\left(\frac{\partial^{2}}{\partial x^{2}}+\frac{\partial^{2}}{\partial z^{2}}\right) \psi+k^{2} n^{2}(x, z) \psi=0
$$

*The electrons in a synchrotron orbit in a horizontal plane. Accordingly, (at least for bending magnet beamlines) their acceleration is also in a horizontal direction and the emitted X-rays are linearly polarised in the horizontal plane. 


\subsubsection{The symmetric slab waveguide}

As the most simple one-dimensionally confining waveguide, we now consider propagation of X-rays in a slab waveguide consisting of a guiding layer with refractive index $n_{1}$ and thickness $d$ sandwiched between two semi-infinite cladding regions of refractive index $n_{2}<$ $n_{1}$. According to Fig. 2.2, we chose our system of coordinates in a way that $x$ denotes the direction of beam propagation while the refractive index $n$ is given as a function of $z$ :

$$
n(z)= \begin{cases}n_{1}, & \text { if }-d<z<0 \\ n_{2}, & \text { else. }\end{cases}
$$

Let us initially ignore absorption and assume the refractive index to be real. For the electric field inside the waveguide we use the ansatz

$$
\psi(x, z)=\psi(z) \exp (-i \beta x)
$$

where $\beta$ is called the propagation constant. Applying the two-dimensional Helmholtz equation 2.12 to this ansatz yields the one-dimensional reduced wave equation for $\psi(z)$ :

$$
\frac{\mathrm{d}^{2}}{\mathrm{~d} z^{2}} \psi(z)+\left(n^{2} k^{2}-\beta^{2}\right) \psi(z)=0 .
$$

Since $\psi$ represents the horizontal component of the electric field, we are considering the socalled transverse electric (TE) modes of the waveguide. This requires $\psi(z)$ to be continuous at the interfaces [Mar74]. Furthermore, for guided modes we demand that the electric field vanishes far inside the cladding, i. e. we demand that $\psi(z)$ approaches zero in the limit $|z| \rightarrow \infty$. The solutions of the wave equation satisfying these boundary conditions are given by

$$
\psi(z)= \begin{cases}B \exp (-\gamma z), & \text { if } z \geq 0, \\ B \cos \kappa z+C \sin \kappa z, & \text { if } 0>z>-d \\ (B \cos \kappa d+C \sin \kappa d) \times \exp [\gamma(z+d)], & \text { if } z \leq-d,\end{cases}
$$

where $B$ and $C$ are constants and

$$
\begin{gathered}
\kappa^{2}=k^{2} n_{1}{ }^{2}-\beta^{2}, \\
\gamma^{2}=\beta^{2}-n_{2}{ }^{2} k^{2}=\left(n_{1}{ }^{2}-n_{2}{ }^{2}\right) k^{2}-\kappa^{2} .
\end{gathered}
$$

For TE modes, $\mathrm{d} \psi / \mathrm{d} z$ also has to be continuous at the interfaces Mar74] and we obtain the following system of equations

$$
\begin{gathered}
\gamma B+\kappa C=0 \\
(\kappa \sin \kappa d-\gamma \cos \kappa d) B+(\gamma \sin \kappa d+\kappa \cos \kappa d) C=0 .
\end{gathered}
$$




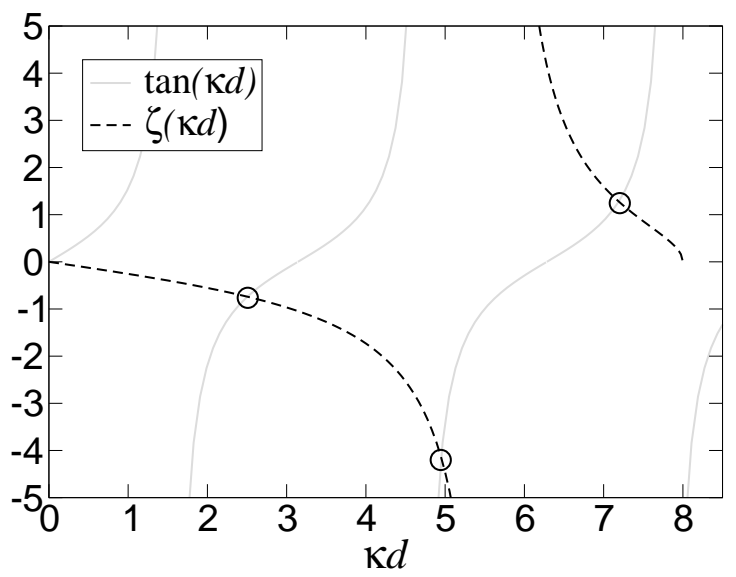

Figure 2.3: Graphical solution of the characteristic equation 2.22 for a waveguide parameter $V=8$. Guided mode solutions are found where the two curves cross. Here, we find 3 guided modes as indicated by the circles.

This system is solved when the system determinant vanishes. We thus find the following characteristic equation

$$
\gamma(\kappa \cos \kappa d+\gamma \sin \kappa d)-\kappa(\kappa \sin \kappa d-\gamma \cos \kappa d)=0
$$

The characteristic equation can also be written in the form

$$
\tan \kappa d=\underbrace{2 \kappa \gamma /\left(\kappa^{2}-\gamma^{2}\right)}_{=: \zeta(\kappa d)} .
$$

Guided modes are found by solving this transcendental equation numerically. Note that the function $\zeta(\kappa d)$ on the right-hand side ends at $\kappa d=\sqrt{n_{1}^{2}-n_{2}^{2}} k d$ since according to Eq. 2.18 $\gamma$ becomes imaginary for larger $\kappa d$. This value is known as the waveguide parameter

$$
V:=\sqrt{\left(n_{1}^{2}-n_{2}^{2}\right)} k d \approx \sqrt{2 \delta_{2}-2 \delta_{1}} k d .
$$

Fig. 2.3 shows a graphical solution of Eq. 2.22) for illustration. The parameter $V$ describes the characteristic behaviour of a waveguide. In particular, it uniquely determines the number of solutions to the characteristic equation (2.22), i. e. the number of guided modes Mar74:

$$
N=\left[\frac{V}{\pi}\right]_{\text {int }} .
$$

Here, [ ] int indicates that the term is rounded up to the next integer. Accordingly, a planar waveguide supports only one guided mode if $d$ is smaller than a critical thickness $W$ given by

$$
W=\frac{\lambda}{\sqrt{2 \delta_{2}-2 \delta_{1}}} .
$$

Apart from multiplicative constants, the solutions of the characteristic equation 2.22 uniquely determine the eigen functions denoted $\psi_{m}$. For convenience, we may choose these 


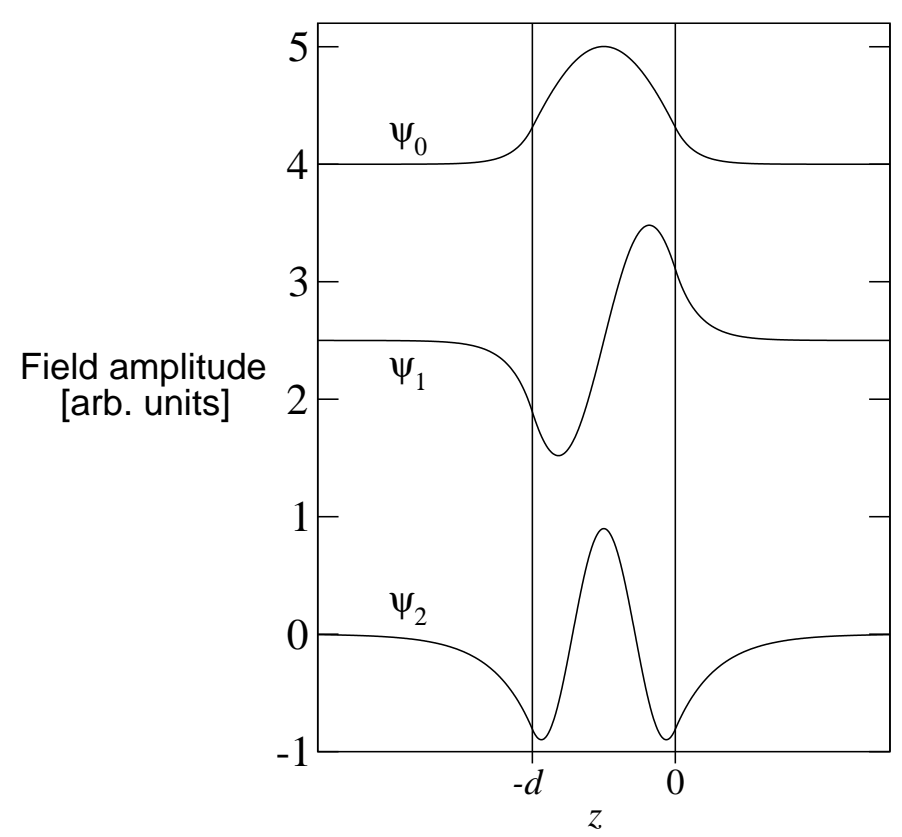

Figure 2.4: Field amplitude of the eigen functions $\psi_{0}, \psi_{1}$, and $\psi_{2}$ of a planar waveguide supporting 3 guided modes. The curves are calculated for $V=8$ and shifted for clarity.

constants to satisfy that the eigen functions are normalised:

$$
\left\|\psi_{m}\right\|=\int\left|\psi_{m}(z)\right|^{2} \mathrm{~d} z=1
$$

For illustration, the eigen functions of a waveguide with $V=8$ supporting 3 guided modes are plotted in Fig. 2.4 .

\subsubsection{Direct coupling}

When an X-ray wave hits the front face of a waveguide, one or more guided modes are excited. Typically, attenuation in the cladding is much stronger than in the guiding core and radiation not contributing to guided modes is quickly damped out. Hence, sufficiently far away from the entrance the scalar wave field is given by a superposition of guided modes $\psi_{m}$ :

$$
\psi(x, z)=\sum_{m=0}^{N-1} c_{m} \psi_{m}(z) \exp \left(-i \beta_{m} x\right) .
$$

The $\beta_{m}$ denote the propagation constants of the individual guided modes and the coefficients $c_{m}$ are given by the projection of the incident field $\psi_{\text {in }}(z)$ onto the respective eigen functions $\left[\mathrm{BDD}^{+}\right.$02, $\left.\mathrm{ST} 91\right]$ :

$$
c_{m}=\int \psi_{\text {in }}(z) \psi_{m}(z) \mathrm{d} z .
$$

We may account for attenuation of the guided modes by introducing an effective linear attenuation coefficient $\mu_{m}$ weighted with respect to field intensity [FS05]:

$$
\mu_{m}=\int \mu(z)|\psi(z)|^{2} \mathrm{~d} z
$$


$\mu(z)$ is given by

$$
\mu(z)= \begin{cases}\mu_{\mathrm{cl}}, & \text { in the cladding, } \\ \mu_{\mathrm{gc}}, & \text { in the guiding core, }\end{cases}
$$

where $\mu_{\mathrm{cl}}$ and $\mu_{\mathrm{gc}}$ denote the linear attenuation coefficients of the cladding and the guiding core, respectively. The resulting wave field is given by

$$
\psi(x, z)=\sum_{m=0}^{N-1} c_{m} \psi_{m}(z) \exp \left[\left(-i \beta_{m}-\mu_{m} / 2\right) x\right] .
$$

In contrary to other common coupling techniques like resonant beam coupling $\left[\mathrm{FSD}^{+} 93\right.$,

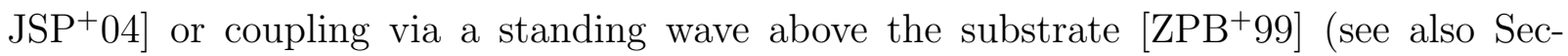
tion 6.3), this technique does not allow for a selective excitation of individual modes of a multi-mode waveguide.

\subsection{Two-dimensionally confining waveguides}

This section introduces cylinder waveguides as a simple model for two-dimensionally confining waveguides. Exact solutions for the eigen functions of optical fibres are well-known Sni61], but since they are quite complicated we will restrict ourselves to the approximation of the weakly-guiding fibre [Glo71, Glo79]. This approximation can be applied when the difference between the refractive indices in the guiding core and in the cladding is small compared to 1 , which is naturally fulfilled for X-rays.

The waveguides used in the experiments presented in the following chapters are approximately of rectangular shape. However, exact analytical solutions for rectangular waveguides have not been given yet. The available approximations [Mar71, $\mathrm{Pfe} 02$ ] ignore some contributions of radiation in the cladding and may thus underestimate absorption in particular. Therefore, rectangular waveguides are analysed here by means of finite-difference field calculations presented in Chapter 3 .

\subsubsection{The weakly-guiding fibre}

We now consider the electric field in a cylinder waveguide with a guiding core radius $a$ (see Fig 2.5.) The refractive indices are $n_{1}$ in the guiding core and $n_{2}$ in the cladding. Like in the case of slab waveguides we once again consider linearly polarised (LP) modes. We introduce cylindrical coordinates $(r, \varphi)$ with

$$
y=r \cos \varphi, \quad z=r \sin \varphi
$$

and denote the eigen functions by $\psi_{\ell m}$ corresponding to the $\mathrm{LP}_{\ell m}$ modes introduced by Gloge in 1971 [Glo71]. The ansatz

$$
\psi(x, r, \varphi)=\psi_{\ell m}(r, \varphi) \exp (-i \beta x)
$$




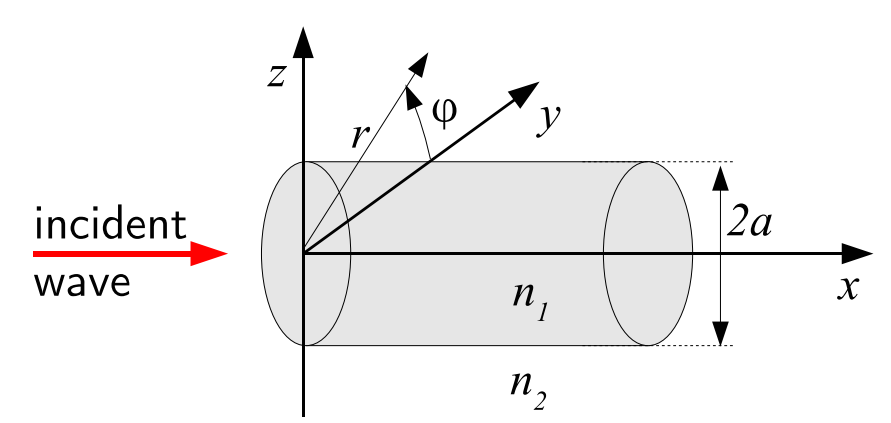

Figure 2.5: Schematic of a fibre waveguide.

in analogy to Eq. 2.14 then yields Glo71, Mar74, Yeh90]

$$
\psi_{\ell m}(r)=B_{\ell m} \begin{cases}\cos (\ell \varphi) \frac{J_{\ell}\left(u_{m} r / a\right)}{J_{\ell}\left(u_{m}\right)}, & \text { for } r<a, \\ \cos (\ell \varphi) \frac{K_{\ell}\left(w_{m} r / a\right)}{K_{\ell}\left(w_{m}\right)}, & \text { for } r \geq a .\end{cases}
$$

$J_{\ell}$ denotes a Bessel function of the first kind of order $\ell$ and $K_{\ell}$ denotes a modified Bessel function of the second kind of order $\ell$ [Wei03]. The $B_{\ell m}$ are constants chosen to satisfy normalisation. Note, that there are also modes where the $\cos (\ell \varphi)$ term in Eq. 2.34) is replaced by $\sin (\ell \varphi)$. However, we will always assume that the incident wave field is given by a plane wave. Without loss of generality, we may assume that the wave vector of the incident plane wave is in the $(x, y)$ plane. Then, for symmetry reasons, we find $\psi(x, z)=\psi(x,-z)$ or $\psi(r, \varphi)=\psi(r,-\varphi)$ and modes with the $\sin (\ell \varphi)$ term are not excited. The parameters $u_{m}$ and $w_{m}$ are obtained as solutions of the characteristic equation [Glo71]

$$
u_{m} \frac{J_{\ell-1}\left(u_{m}\right)}{J_{\ell}\left(u_{m}\right)}=-w_{m} \frac{K_{\ell-1}\left(w_{m}\right)}{K_{\ell}\left(w_{m}\right)}
$$

with $w_{m}=\sqrt{V^{2}-u_{m}^{2}}$. We define the dimensionless waveguide parameter of a fibre by [Mar74, Glo71]

$$
V:=k a \sqrt{n_{1}^{2}-n_{2}^{2}}
$$

For an incident plane wave at normal incidence, symmetry requires $\ell=0$ and the characteristic equation reads

$$
u_{m} \frac{J_{1}\left(u_{m}\right)}{J_{0}\left(u_{m}\right)}=w_{m} \frac{K_{1}\left(w_{m}\right)}{K_{0}\left(w_{m}\right)} .
$$

The total number of guided modes is again determined by $V$. If $V$ is less than the first zero of the Bessel function $J_{0}$, the waveguide supports only one linearly polarised guided mode for each polarisation direction. The maximum diameter of a single-mode fibre is thus given by

$$
2 a_{c}=\frac{2 V_{1}}{\pi} \frac{\lambda}{2 \theta_{c}} \approx 1.53 \mathrm{~W}
$$

where $V_{1} \approx 2.40$ is the first zero of the Bessel function $J_{0}$ and $W$ is the corresponding maximum thickness of a single-mode slab waveguide. Far away from the entrance, the 


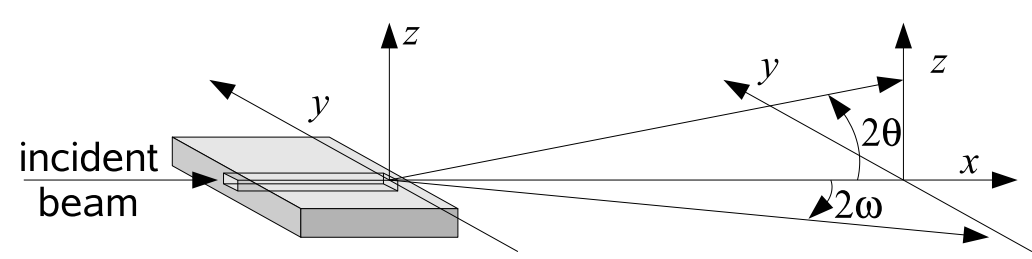

Figure 2.6: The Fraunhofer diffraction pattern is a functions of the angles $2 \theta$ and $2 \omega$ included between the exiting beam and the $x$ axis in the $(x, z)$ and $(x, y)$ plane, respectively.

electric field is given as a linear combination of guided modes:

$$
\psi(r, x)=\sum_{m} c_{m} \psi_{0 m}(r) \exp \left[\left(-i \beta_{m}-\mu_{m} / 2\right) x\right] .
$$

The coefficients $c_{m}$ are given by the overlap integral of the corresponding eigen function and the incident electric field $\psi_{\text {in }}$

$$
c_{m}=2 \pi \int_{0}^{\infty} \psi_{0 m}(r) \psi_{\text {in }}(r) r \mathrm{~d} r
$$

and $\beta_{m}=\sqrt{n_{1}^{2} k^{2}-u_{m}^{2} / a^{2}}$ are the propagation constants of the individual guided modes. $\mu_{m}$ represents the linear attenuation coefficient weighted with respect to intensity given by

$$
\mu_{m}=2 \pi \int_{0}^{\infty}\left|\psi_{0 m}(r)\right|^{2} \mu(r) r \mathrm{~d} r
$$

\subsection{The Fraunhofer diffraction pattern}

Although it seems very difficult to measure the electromagnetic field inside an X-ray waveguide directly, one may deduce this information from the diffraction pattern measured far downstream of the waveguide. In the small-angle approximation, intensity of the Fraunhofer (or far-field) diffraction pattern of a one-dimensionally confining waveguide is given by BW87]

$$
I(2 \theta) \propto\left|\int \psi(z) \exp (i k 2 \theta z) \mathrm{d} z\right|^{2}
$$

as a function of the exit angle $2 \theta$ (see Fig. 2.6). In the same way, the pattern measured behind a two-dimensionally confining waveguide is given as a function of the angles $2 \theta$ and $2 \omega$ (as defined in Fig. 2.6) by

$$
I(2 \theta, 2 \omega) \propto\left|\int \psi(y, z) \exp (i k 2 \theta z) \exp (-i k 2 \omega y) \mathrm{d} y \mathrm{~d} z\right|^{2} .
$$

The far-field intensity pattern thus represents the square modulus of the Fourier transform of the field at the end face of the waveguide. A decreasing thickness of the guiding core thus increases the divergence of the exiting beam. 


\subsection{The parabolic wave equation}

Consider a wave propagating along a direction close to the direction of the $x$ axis, i.e. with a wave vector $\boldsymbol{k}$ including an angle $\theta$ less than approximately the critical angle of total reflection with the $x$ axis. Evidently, $\psi$ then oscillates very quickly in $x$ direction, namely on the length scale of the X-ray wavelength. Thus, a straightforward numerical approach like the finite-difference calculations presented in Chapter 3 requires a very fine grid spacing and an accordingly large number of computational operations. To overcome this problem, $\psi$ is written as the product of a factor $\exp (-i k x)$, describing the propagation of a plane wave in free space in $x$ direction, and a function $u$, describing the modulation of the wave field by the waveguide:

$$
\psi(x, y, z)=u(x, y, z) \exp (-i k x) .
$$

Evidently, in $x$ direction $u$ oscillates much more slowly than $\psi$. Consider for instance a plane wave propagating in $x$ direction through a medium with refractive index $1-\delta$. In this case we find $u=u_{0} \exp (i k \delta x)$ and thus we expect that the grid can be made coarser by a factor of $\delta^{-1}$ which may correspond to more than $10^{5}$. Ignoring the second order partial derivative of $u$ in $x$-direction, after insertion of Eq. 2.44 in the Helmholtz equation 2.11) we get

$$
-2 i k \frac{\partial u}{\partial x}+\left(\frac{\partial^{2}}{\partial y^{2}}+\frac{\partial^{2}}{\partial z^{2}}\right) u+k^{2}\left(n^{2}-1\right) u=0 .
$$

This equation is known as the parabolic wave equation. It was introduced by Leontovich and Fock in the late 1940s for radio wave propagation [Foc65] and has has already been applied to the study of X-ray diffraction optics [KPV95] and X-ray waveguides [BKvdV03]. For convenience we define

$$
A:=-\frac{i}{2 k} \quad \text { and } \quad F(x, y, z):=-\frac{i k}{2}\left(n^{2}(x, y, z)-1\right)
$$

and the parabolic wave equation reads

$$
\frac{\partial u}{\partial x}=A\left(\frac{\partial^{2} u}{\partial y^{2}}+\frac{\partial^{2} u}{\partial z^{2}}\right)+F(x, y, z) u
$$

\subsection{The limits of confinement}

Although any symmetric waveguide with a finite guiding layer thickness supports at least one guided mode [Mar74], X-rays may not be confined arbitrarily by means of waveguides. For extremely small guiding core dimensions, the width of the guided modes becomes significantly larger than the guiding core dimensions due to the evanescent wave in the cladding BKvdV03, KK03. Indeed, it transpires that the minimum achievable beam size is limited by the electron density of the utilised materials rather than by the X-ray wavelength. 
Approximating $n^{2} \approx 1-2 \delta$, the parabolic wave equation for a planar waveguide may be written as

$$
-\frac{i}{k} \frac{\partial u}{\partial x}+\frac{1}{2 k^{2}} \frac{\partial^{2} u}{\partial z^{2}}+\delta u=0 .
$$

This equation has the same form like the one-dimensional time-dependent Schrödinger equation for the wave function $\Psi$ describing a particle of mass $m$ in a one-dimensional potential $U(x)$ :

$$
i \hbar \frac{\partial \Psi}{\partial t}=-\frac{\hbar^{2}}{2 m} \frac{\partial^{2} \Psi}{\partial x^{2}}+U(x) .
$$

Here, $\hbar=h / 2 \pi \approx 1.05 \times 10^{-34}$ Js denotes the reduced Planck constant. With $t=-x$, $\hbar=k^{-1}, m=1$, and $U=\delta$ the problem of finding the guided modes of a waveguide can be transformed to the well-known problem of finding the bound states of a particle inside a potential well $U(x)$ with maximum height $U_{0}=\delta$ [LL79]. For a bound state the particle momentum $p$ is limited by $p^{2} / 2 m \leq \delta$ and the corresponding maximum uncertainty is $\Delta_{p}=\sqrt{2 m U_{0}}$. According to the Heisenberg uncertainty principle

$$
\Delta_{x} \Delta_{p} \geq \hbar
$$

we find the lower limit of the position uncertainty $\Delta_{x}$ given by

$$
\Delta_{x} \geq \frac{\hbar}{\sqrt{2 m U_{0}}}=\frac{1}{k \sqrt{2 \delta}}
$$

Bergemann et al. have pointed out that this gives a universal lower limit of the beam size achievable by confinement in X-ray waveguides BKvdV03. It is related to the critical width $W$ of a single-mode planar waveguide by

$$
\Delta_{x} \geq \frac{W}{\pi}
$$

Away from the absorption edges, we may apply Eq. 2.3 and express $W$ as a function of the electron densities $\varrho_{e, 1}$ and $\varrho_{e, 2}$ in the guiding layer and in the cladding, respectively:

$$
W=\sqrt{\frac{\pi}{\left(\varrho_{e, 2}-\varrho_{e, 1}\right) r_{e}}} .
$$

Evidently, highest confinement is achieved using heavy elements in the cladding and light elements (or vacuum) in the guiding core. For example, we find $W \approx 30 \mathrm{~nm}$ for a PMMA guiding layer in silicon (see Section 6.2 ) or $W \approx 9 \mathrm{~nm}$ for a vacuum guiding layer in gold. A further decrease of the guiding core dimensions strongly enhances the evanescent wave field in the cladding without significantly decreasing the full width at half maximum of the electric field intensity. Consequently, there is a strong increase of the portion of mode power propagating in the cladding [Glo71] and hence strong absorption.

Note that close to the absorption edges, $\delta$ is smaller than the value given in Eq. (2.3) and the critical width is even larger than the value given here. 


\subsubsection{Comparison to the limits of other focusing optics}

It is currently under debate whether the universal limit of $W$ may apply to all X-ray focusing optics. Evidently, the limit cannot be overcome by tapered waveguides $\left[\mathrm{ZBP}^{+} 00\right]$ or glass capillaries [BHT94]. The critical width $W$ may also be written as a function of the critical angle of total external reflection $\theta_{c}$ :

$$
W=\frac{\lambda}{2 \theta_{c}} \text {. }
$$

The maximum spatial resolution $s$ of an optical device depends on the numerical aperture NA [Vog97:

$$
s \approx \frac{\lambda}{2 \mathrm{NA}}
$$

Since the numerical aperture of a waveguide is simply the critical angle $\theta_{c}$, we find that the maximum resolution achievable in waveguide-based imaging experiments is given by the critical width $W$.

A comparable situation is found for refractive lenses [SKSL96, LSK ${ }^{+}$05]. The numerical aperture of a refractive X-ray lens is limited to $\sqrt{2 \delta}=\theta_{c}$ as well [PV98, Suz04]. However, Schroer et al. have proposed an arrangement of multiple lenses with decreasing diameters, called adiabatic focusing lenses. In the approximation of planar diffraction masks, they have calculated a larger numerical aperture and correspondingly higher spatial resolution SL05].

The numerical aperture of reflective optics is in practice also limited by the critical angle, since sufficient reflectivity is usually only found at lower grazing angles. However, this limit could possibly be overcome using Bragg reflection of multilayer mirrors providing sufficient reflectivity also at grazing angles above $\theta_{c}\left[\mathrm{HCR}^{+} 05\right]$.

So far, smallest beam dimensions have been obtained by planar X-ray waveguides [Pfe02] and Fresnel zone plates [CHL $\left.{ }^{+} 05\right]$. However, the focal spot sizes of state-of-the-art $\mathrm{X}$-ray optics, in particular in the hard X-ray regime, have not reached $W$ yet. Recent experiments have demonstrated focal spot sizes of $47 \times 55 \mathrm{~nm}^{2}$ using compound refractive lenses [SKP $\left.{ }^{+} 05\right], 36 \times 48 \mathrm{~nm}^{2}$ using mirror optics [MMY $\mathrm{MM}^{+}$], and $15 \mathrm{~nm}$ using a Fresnel zone plate in the soft $\mathrm{X}$-ray regime $\left.\mathrm{CHL}^{+} 05\right]$.

A unique property of X-ray waveguides compared to focusing lenses or mirrors is that the size of the exiting beam is independent of the coherence properties of the incident beam, i. e. the size of an incoherent source (Fig. 2.7). Indeed, all lenses provide a demagnified image of the source. The minimum focal spot size is then limited by the source dimensions $\sigma$, the focal distance $f$, and the distance $L$ between the lens and the source (Fig. 2.7(a) $)$ :

$$
s \geq \frac{f}{L} \sigma
$$

Typical source sizes of undulator beamlines are $30 \mu \mathrm{m}$ in vertical and $120-800 \mu \mathrm{m}$ in horizontal direction. The focal spot of a lens with a focal distance of $f=0.1 \mathrm{~m}$ at a typical 


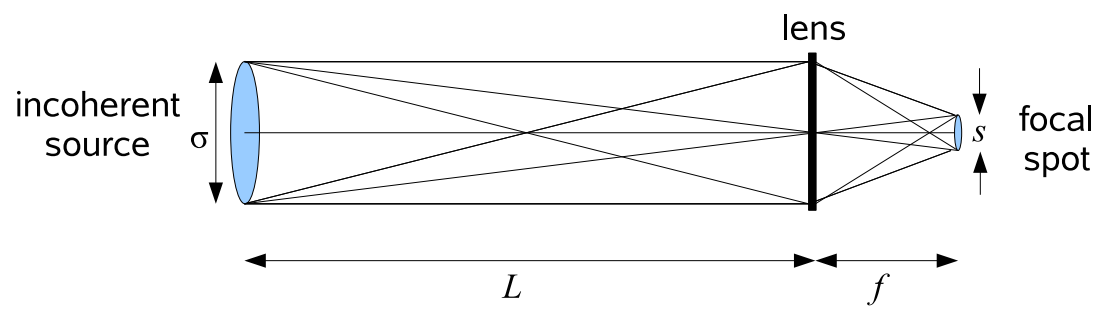

(a)

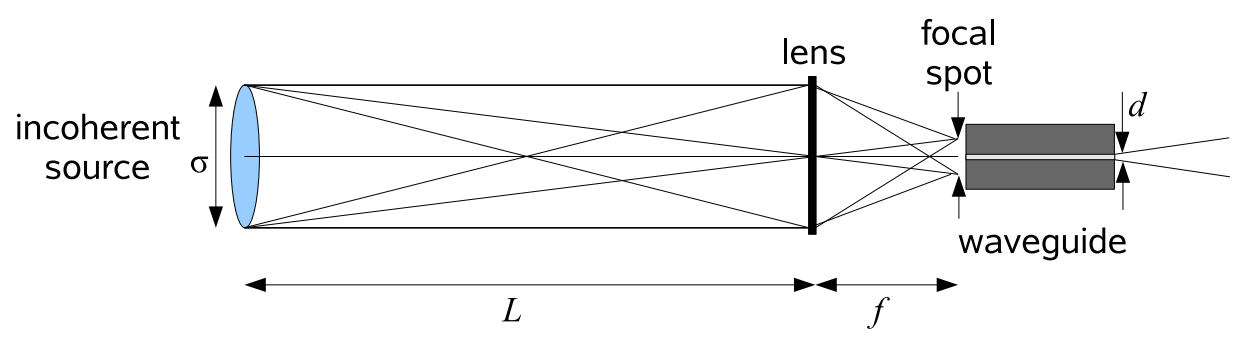

(b)

Figure 2.7: The focal spot of a lens is a demagnified image of the incoherent source. Accordingly, the minimum focal spot size is limited to $\sigma f / L$ (a). The dimensions of the beam exiting a waveguide are independent of the source size and depend only on the guiding core dimensions $d$. A waveguide placed in the focal spot of a lens (b) can thus significantly further reduce the beam size.

source-to-lens distance of $40 \mathrm{~m}$ may then not be smaller than $300 \mathrm{~nm}$ in horizontal direction. Accordingly, the smallest focal spot dimensions are measured at extremely long beamlines with distances of up to $1 \mathrm{~km}$ to the source [MMY $\left.{ }^{+} 05\right]$. There have also been attempts to re-focus a waveguide beam in order to overcome focal spot limitations due to the source size $\left[\mathrm{LBM}^{+} 06\right]$.

In the experiments presented in the later chapters, we have combined both a prefocusing device (in this instance Kirkpatrick-Baez mirrors [KB48, $\mathrm{HCL}^{+} 03$ ]) and an X-ray waveguide. The pre-focusing device provides a high density of flux while the waveguide significantly further reduces the beam size (Fig. 2.7(a)).

\subsection{Dimensionless coordinates}

In order to find results independent of a special choice of materials or photon energy, we consider a vacuum waveguide in a homogeneous material of refractive index $n$. With the critical width $W$ given in Eq. 2.25) and approximating $n^{2}=(1-\delta-i b)^{2} \approx 1-2(\delta+i b)$, the parabolic wave equation reads

$$
\frac{\partial u}{\partial(\delta k x)}= \begin{cases}-\frac{i}{\pi^{2}}\left(\frac{\partial^{2} u}{\partial(y / W)^{2}}+\frac{\partial^{2} u}{\partial(z / W)^{2}}\right)+\left(i-\frac{b}{\delta}\right) u, & \text { in the cladding } \\ -\frac{i}{\pi^{2}}\left(\frac{\partial^{2} u}{\partial(y / W)^{2}}+\frac{\partial^{2} u}{\partial(z / W)^{2}}\right), & \text { in vacuum }\end{cases}
$$


This suggests the introduction of the following dimensionless variables

$$
X:=\delta k x, \quad Y:=y / W, \quad \text {, and } \quad Z:=z / W .
$$

The parabolic wave equation then reads

$$
\frac{\partial u}{\partial X}= \begin{cases}-\frac{i}{\pi^{2}}\left(\frac{\partial^{2} u}{\partial Y^{2}}+\frac{\partial^{2} u}{\partial Z^{2}}\right)+\left(i-\frac{b}{\delta}\right) u, & \text { in the cladding } \\ -\frac{i}{\pi^{2}}\left(\frac{\partial^{2} u}{\partial Y^{2}}+\frac{\partial^{2} u}{\partial Z^{2}}\right), & \text { in vacuum }\end{cases}
$$

Written in these coordinates, the X-ray wavelength and the absolute values of the real and imaginary part of the refractive index do not explicitly appear in the parabolic wave equation. Accordingly, a solution of Eq. 2.59) gives a general result that applies to any aperture with a given core diameter $D$ measured in units of $W$ and a given $b / \delta$ ratio. Note that away from the absorption edges we have $\delta=\lambda^{2} \varrho_{e} r_{e} / 2 \pi$ (see Eq. 2.3p). The critical width $W$ is therefore practically independent of the X-ray wavelength [BKvdV03]. 


\section{Finite-difference field calculations}

To determine coherence and transmission properties of $X$-ray waveguides it is necessary to calculate the electric field inside and in particular at the end of the waveguide. As shown in Chapter 2, there are exact analytical solutions for cylinder and slab waveguides. But many structures [Jar05, See04] that may be used as two-dimensionally confining waveguides have cross-sections that do not allow for an exact analytical solution. Even for some quite simple geometries like a rectangular or triangular cross-section, exact analytical solutions have not yet been calculated*. Furthermore, the cross-section may change along the waveguide. For example, tapered waveguides $\left[\mathrm{ZBP}^{+} 00\right]$ may be used as focusing devices, or $Y$ branches may provide two coherent beams for off-axis holography experiments.

This chapter describes how the electric field inside one-dimensionally and two-dimensionally confining waveguides can be determined by means of finite-difference calculations. Due to the large ratio of the waveguide dimensions to the X-ray wavelength, a straightforward numerical approach based on the Helmholtz equation is a formidable task. Therefore, the calculations are based on the parabolic wave equation introduced in Section 2.6.

We start with a discretisation of the electric field and the introduction of appropriate boundary conditions (Section 3.1). First, we consider the simpler case of one-dimensionally confining waveguides (Section 3.2). A finite-difference algorithm based on the CrankNicolson scheme [Tho95] is presented and the results are verified by comparison to analytical results, namely to Fresnel reflectivity and to the planar slab waveguide. Furthermore, it is shown that the calculations may also yield the guided modes. Section 3.3 then presents a finite-difference algorithm based on the Peaceman-Rachford scheme [PR55, Tho95], enabling field calculations for two-dimensionally confining waveguides. Again, the results are verified by comparison to analytical theory, in this case to the weakly guiding optical fibre [Mar74, Glo71].

\subsection{Discretisation and boundary conditions}

For a numerical solution of the parabolic wave equation 2.47), we calculate $u(x, y, z)$ on a grid of $2 M_{x}+1, M_{y}+1$, and $M_{z}+1$ points equidistantly spaced by $\Delta x, \Delta y$, and $\Delta z$ in the respective directions (Fig. 3.1). We furthermore define

$$
u_{j k}^{n}:=u\left(x_{n}, y_{j}, z_{k}\right)
$$

*Analytical approximations for rectangular waveguides do exist [Mar74, Pfe02, but these approximations ignore the electric field in the corners of the cladding. Thus, they may fail, particularly in the case of single-mode waveguides. 


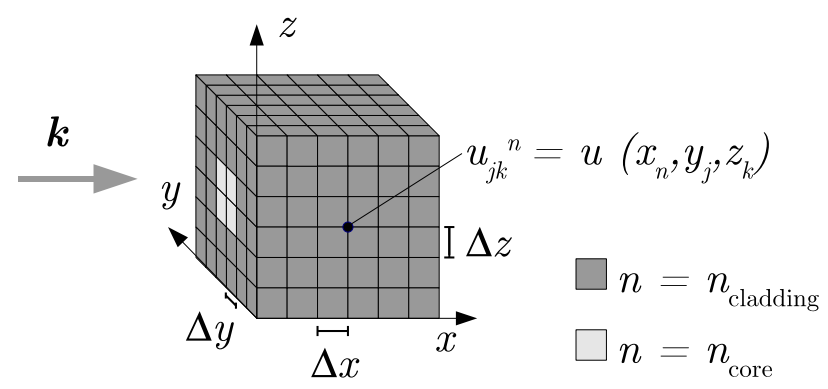

Figure 3.1: The electric field is calculated on a grid equidistantly spaced by $\Delta x, \Delta y$ and $\Delta z$ in the respective directions. The wave vector $\boldsymbol{k}$ of the incident plane wave is assumed to be approximately parallel to the $x$ axis. Note that the point of origin is located outside the guiding core to simplify notation.

with

$$
\begin{aligned}
x_{n} & :=n \Delta x, & n & =0,1 / 2,1,1 \frac{1}{2}, 2, \ldots, M_{x}, \\
y_{j} & :=j \Delta y, & j & =0,1,2, \ldots, M_{y}, \\
z_{k} & :=k \Delta z, & k & =0,1,2, \ldots, M_{z} .
\end{aligned}
$$

Evidently, $\Delta y$ and $\Delta z$ have to be small compared to the critical width $W$ given in Eq. 2.25). Since the guided modes oscillate according to $\exp (-i \beta x)$ in $\psi$ and thus according to $\exp [-i(\beta-k) x]$ in $u, \Delta x$ has to be small compared to $(\beta-k)^{-1}$. Typical values for hard X-rays are $\Delta y=\Delta z=1 \mathrm{~nm}$ and $\Delta x=100 \mathrm{~nm}$.

We now give the following boundary conditions: At the entrance of the waveguide, the fields $\psi$ and $u$ are given by an incident wave with amplitude $\psi_{\text {in }}(y, z)$ :

$$
u(x=0, y, z)=\psi_{\text {in }}(y, z) .
$$

Within this thesis, $\psi_{\text {in }}$ is always assumed to be given by a plane wave*. Far away from the guiding core, we expect that the field is not significantly disturbed by the waveguide and hence is given by a damped plane wave propagating with respect to the refractive index of the cladding. In the special case where the incident wave hits the waveguide at normal incidence, we find $\psi_{\text {in }}(y, z)=$ const. $=\psi_{\text {in }}$ and

$$
u\left(x_{n}, y_{j}, z_{k}\right)=\psi_{\text {in }} \exp \left[-i\left(n_{\mathrm{cl}}-1\right) k x_{n}\right], \quad \text { for } j \in\left\{0, M_{y}\right\} \text { or } k \in\left\{0, M_{z}\right\}
$$

where $n_{\mathrm{cl}}$ denotes the complex refractive index of the cladding. Thus, the parabolic wave equation (2.47) together with equations (3.5) and (3.6) give a well-posed initial-boundaryvalue problem. Eq. (3.5) determines all values for $u_{j k}^{n=0}$ and we show in the next sections how $u_{j k}^{n+1}$ can be calculated when $u_{j k}^{n}$ is known for all $j$ and $k$.

* Note that an exact solution would also take into account small disturbances of the incident wave upstream of the waveguide. However, these disturbances are very small, so we can ignore them. 


\subsection{One-dimensionally confining waveguides}

For one-dimensionally confining waveguides it is sufficient to solve the parabolic wave equation in two dimensions. Dropping any $y$ dependence, the parabolic wave equation reads

$$
\frac{\partial u}{\partial x}=A \frac{\partial^{2} u}{\partial z^{2}}+F(x, z) u
$$

In analogy to Eq. (3.5) and (3.6) we give the following boundary conditions

$$
\begin{gathered}
u(0, z)=u_{0}(0, z), \\
u\left(x, z_{0}\right)=u_{0}\left(x, z_{0}\right), \\
u\left(x, z_{M_{z}}\right)=u_{0}\left(x, z_{M_{z}}\right),
\end{gathered}
$$

where $u_{0}(x, z)$ describes an incident plane wave or a damped plane wave propagating in the cladding far away from the guiding core, respectively. This initial-boundary-value problem is solved numerically using a finite-difference scheme as described in [SO91]. The scheme is based on the Crank-Nicolson scheme, which is second order accurate in $\Delta x$ and $\Delta z$ [Tho95]. Away from the boundaries, we replace the partial derivatives in Eq. (3.7) by the following finite-difference expressions:

$$
\begin{aligned}
\frac{\partial u}{\partial x} & \rightarrow \frac{u_{k}^{n+1}-u_{k}^{n}}{\Delta x}, \\
A \frac{\partial^{2} u}{\partial z^{2}} & \rightarrow \frac{A}{2}\left(\frac{u_{k-1}^{n}-2 u_{k}^{n}+u_{k+1}^{n}}{\Delta z^{2}}+\frac{u_{k-1}^{n+1}-2 u_{k}^{n+1}+u_{k+1}^{n+1}}{\Delta z^{2}}\right), \\
F(x, z) u & \rightarrow \frac{F_{k}^{n+\frac{1}{2}}}{2}\left(u_{k}^{n}+u_{k}^{n+1}\right)
\end{aligned}
$$

with $u_{k}^{n}=u\left(x_{n}, z_{k}\right)$ and $F_{k}^{n+\frac{1}{2}}=F\left(x_{n+\frac{1}{2}}, z_{k}\right)$. Thus, we find the finite-difference equation

$$
\frac{u_{k}^{n+1}-u_{k}^{n}}{\Delta x}=\frac{A}{2}\left(\frac{u_{k-1}^{n}-2 u_{k}^{n}+u_{k+1}^{n}}{\Delta z^{2}}+\frac{u_{k-1}^{n+1}-2 u_{k}^{n+1}+u_{k+1}^{n+1}}{\Delta z^{2}}\right)+\frac{F_{k}^{n+\frac{1}{2}}}{2}\left(u_{k}^{n}+u_{k}^{n+1}\right)
$$

For convenience we define

$$
r_{z}:=A \frac{\Delta x}{\Delta z^{2}}, \quad C_{k}^{n+\frac{1}{2}}:=\frac{F_{k}^{n+\frac{1}{2}} \Delta x}{2} .
$$

Eq. (3.14) may now be written in the form

$$
B^{n} \boldsymbol{u}^{n+1}=\boldsymbol{d}^{n}
$$

with

$$
B^{n}=\left(\begin{array}{ccccc}
1+r_{z}-C_{1}^{n+\frac{1}{2}} & -\frac{r_{z}}{2} & 0 & \ldots & \\
-\frac{r_{z}}{2} & 1+r_{z}-C_{2}^{n+\frac{1}{2}} & -\frac{r_{z}}{2} & 0 & \ldots \\
\cdots & & \ddots & & \\
& 0 & -\frac{r_{z}}{2} & 1+r_{z}-C_{2}^{n+\frac{1}{2}} & -\frac{r_{z}}{2} \\
& \cdots & 0 & -\frac{r_{z}}{2} & 1+r_{z}-C_{M_{z}-1}^{n+\frac{1}{2}}
\end{array}\right),
$$




$$
\boldsymbol{u}^{n+1}=\left(\begin{array}{c}
u_{1}^{n+1} \\
u_{2}^{n+1} \\
\vdots \\
u_{M_{z}-2}^{n+1} \\
u_{M_{z}-1}^{n+1}
\end{array}\right)
$$

and

$$
\boldsymbol{d}^{n}=\left(\begin{array}{c}
\frac{r_{z}}{2} u_{0}^{n}+\left(1-r_{z}+C_{1}^{n+\frac{1}{2}}\right) u_{1}^{n}+\frac{r_{z}}{2} u_{2}^{n}+\frac{r_{z}}{2} u_{0}^{n+1} \\
\frac{r_{z}}{2} u_{1}^{n}+\left(1-r_{z}+C_{2}^{n+\frac{1}{2}}\right) u_{2}^{n}+\frac{r_{z}}{2} u_{3}^{n} \\
\vdots \\
\frac{r_{z}}{2} u_{M_{z}-3}^{n}+\left(1-r_{z}+C_{M_{z}-2}^{n+\frac{1}{2}}\right) u_{M_{z}-2}^{n}+\frac{r_{z}}{2} u_{M_{z}-1}^{n} \\
\frac{r_{z}}{2} u_{M_{z}-2}^{n}+\left(1-r_{z}+C_{M_{z}-1}^{n+\frac{1}{2}}\right) u_{M_{z}-1}^{n}+\frac{r_{z}}{2} u_{M_{z}}^{n}+\frac{r_{z}}{2} u_{M_{z}}^{n+1}
\end{array}\right)
$$

Note that the index $n+1$ does not appear for any entry of $B^{n}$. In $\boldsymbol{d}^{n}$, the index $n+1$ only appears for $u_{0}^{n+1}$ and $u_{M_{z}}^{n+1}$ which can be determined from the boundary conditions 3.9 ) and (3.10). Accordingly, Eq. (3.16) allows the determination of the field $\boldsymbol{u}^{n+1}$ from the field $\boldsymbol{u}^{n}$ by solving a system of $M_{z}-1$ linear equations. Since the matrix $B^{n}$ is tridiagonal, this can be done with $\mathcal{O}\left(M_{z}\right)$ operations [Tho95. Starting with $\boldsymbol{u}^{0}$ given by the incident wave, the whole field can be calculated successively within $M_{x}$ steps. The total computational time is thus in the order of $\mathcal{O}\left(M_{x} \times M_{z}\right)$.

The algorithm was implemented using the IDLf programming language and the essential procedure of the source code is given in the Appendix (Section A.4.1). A typical field calculation takes less than one minute on a Linux workstation with a $2.4 \mathrm{GHz}$ processor and 512 MB RAM.

\subsubsection{Comparison to analytical results}

Some typical results of finite-difference field calculations in two dimensions are now presented and compared to analytical theory.

\section{Fresnel Reflectivity}

As a basic test of the finite-difference algorithm, the reflectivity of X-rays on a plane surface is studied. Note that this is not only a test of the algorithm but a test of the applicability of the parabolic wave equation as well. Fig. 3.2 shows the electric field of a Gaussian shaped beam reflected by a Ni surface at a grazing angle of $\theta_{i}=0.2^{\circ}$ calculated for different photon energy $E$. The boundary conditions are a Gaussian beam at $x=0$ and a vanishing field for large $|z|$ values. For $10 \mathrm{keV}, \theta_{i}=0.2^{\circ}$ is within the regime of total external reflection, while it is not for $25 \mathrm{keV}$. This is indeed observed in the calculations: At $E=10 \mathrm{keV}$ we find an intense reflected beam and a standing wave is observed above the surface where

${ }^{*}$ IDL is a registered trademark of Research Systems Inc. 


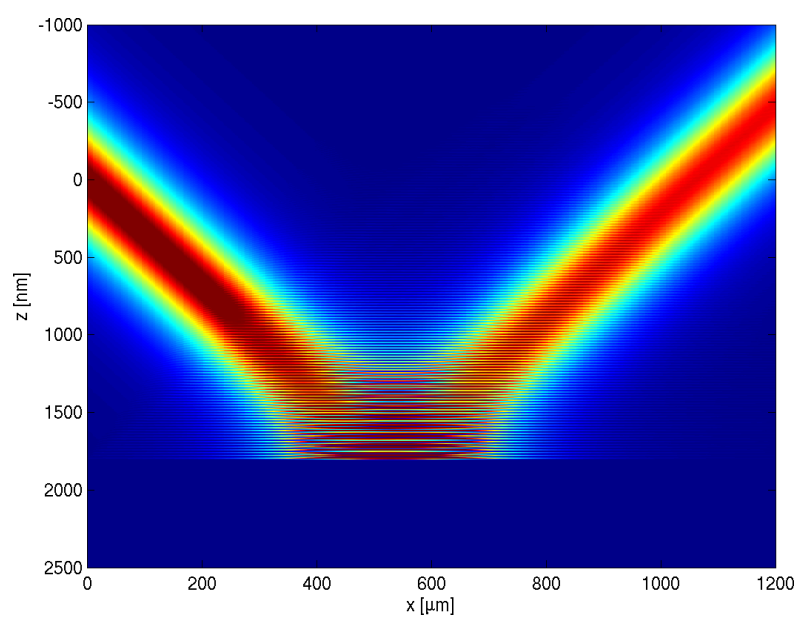

(a) $E=10 \mathrm{keV}$.

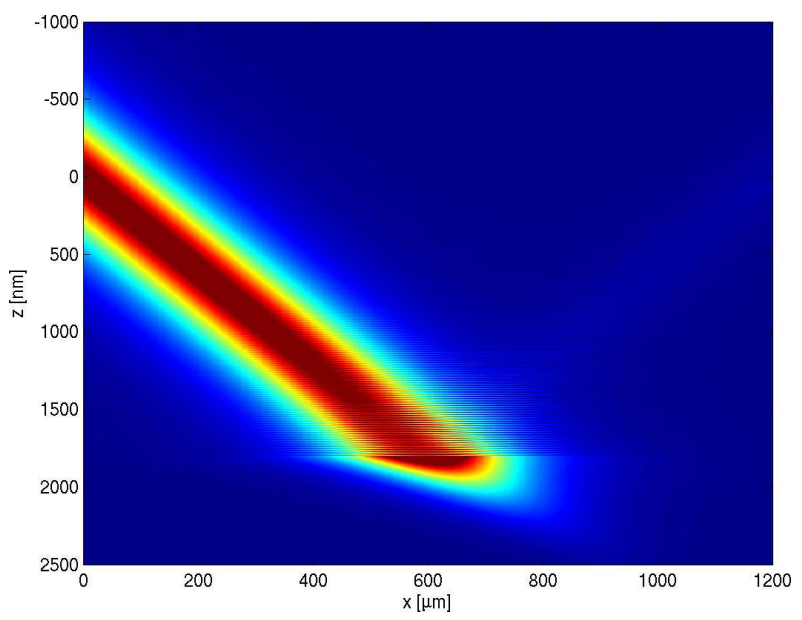

(b) $E=25 \mathrm{keV}$.

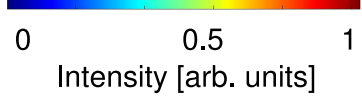

Figure 3.2: Finite-difference field calculation of a Gaussian beam reflected by a Ni surface. At a grazing angle of $0.2^{\circ}$ the calculation for a photon energy $E$ of $10 \mathrm{keV}$ (a) demonstrates the regime of total external reflection while the calculation for $25 \mathrm{keV}$ (b) demonstrates refraction and absorption of the transmitted beam in the substrate.

the incident and the reflected beam interfere. At $E=25 \mathrm{keV}$ the reflected beam is much less intense, but we find a transmitted beam that is quickly absorbed in the Ni substrate.

To quantify the results, intensity reflectivity is calculated and compared to analytical solutions given by the Fresnel equations [ANM01]. For amplitude reflectivity the Fresnel equations yield

$$
r_{F}=\frac{\theta_{i}-\theta_{r}}{\theta_{i}+\theta_{r}}
$$

where $\theta_{i}$ and $\theta_{r}$ denote the grazing angles of the incident and the reflected beam, respectively, related by Snell's law (see Section 2.1). Intensity reflectivity $R_{F}$ is then given by the squared modulus of amplitude reflectivity:

$$
R_{F}=\left|r_{F}\right|^{2}
$$

Fig. 3.3 shows the intensity reflectivity obtained from finite-difference calculations at photon energies in the range $10-25 \mathrm{keV}$ in comparison to the Fresnel solution. The latter was calculated with the IMD software Win98] using exactly the same optical constants. Both results are in excellent agreement in the regime of total external reflection. Note that reflectivity is smaller than 1 in both results, even in the regime of total external reflection. This is due to absorption of the evanescent wave and indicates that this effect is taken into account by the finite-difference calculations, since the complex index of refraction is used. At higher photon energy, the finite-difference calculations yield systematically higher reflectivity. This indicates that the parabolic wave equation may not be applicable any 


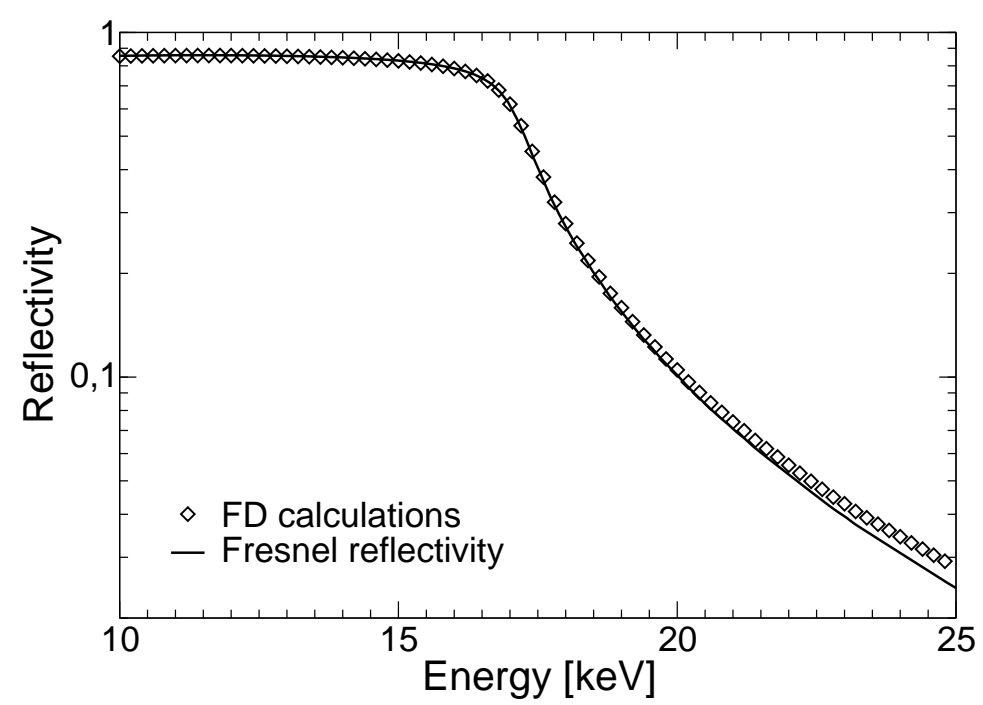

Figure 3.3: Reflectivity of a Gaussian beam on a Ni surface, calculated with the finite-difference (FD) approach and compared to the Fresnel solution (grazing angle $0.2^{\circ}$, no surface roughness). The curves are in excellent agreement in the regime of total external reflection. For higher photon energies, the FD approach yields higher reflectivity.

more. However, propagation in waveguides is naturally in the regime of total external reflection and one may expect reasonable results.

\section{Slab waveguides}

We now consider the propagation of X-rays in planar slab waveguides. According to Section 2.3.2, the electric field inside the waveguide is given as a superposition of guided modes

$$
\psi(x, z)=\sum_{m=0}^{N-1} c_{m} \psi_{m}(z) \exp \left[\left(-i \beta_{m}-\mu_{m} / 2\right) x\right]
$$

where $\psi_{m}$ denotes the eigen functions belonging to the guided modes, $\beta_{m}$ denotes the propagation constant, $\mu_{m}$ is the mode-dependent attenuation coefficient, and $c_{m}$ is given by the overlap integrals of the incident wave and the respective eigen function according to Eq. (2.28). For simplicity, we first consider an incident field given by a plane wave at normal incidence. For symmetry reasons, the overlap integral (2.28) then vanishes for all odd modes* and only even modes are excited.

Fig. 3.4 shows the field intensity determined by both the analytical approach and finite-difference calculations for planar waveguides with a polyimide $\left(\mathrm{C}_{22} \mathrm{H}_{10} \mathrm{~N}_{2} \mathrm{O}_{5}, \rho=\right.$ $1.42 \mathrm{~g} / \mathrm{cm}^{3}$ ) guiding layer in a silicon cladding. Apart from some quickly damped intensity contributions in the cladding visible near the entrance of the waveguid€ both approaches give practically identical results. For a single-mode waveguide $(d=30 \mathrm{~nm})$, we find that the field is basically determined by the damped $\psi_{0}$ mode. In multi-mode waveguides

* Note that even modes are symmetric while odd modes are antisymmetric.

$\dagger$ These contributions may also be interpreted as radiative modes Mar74. 

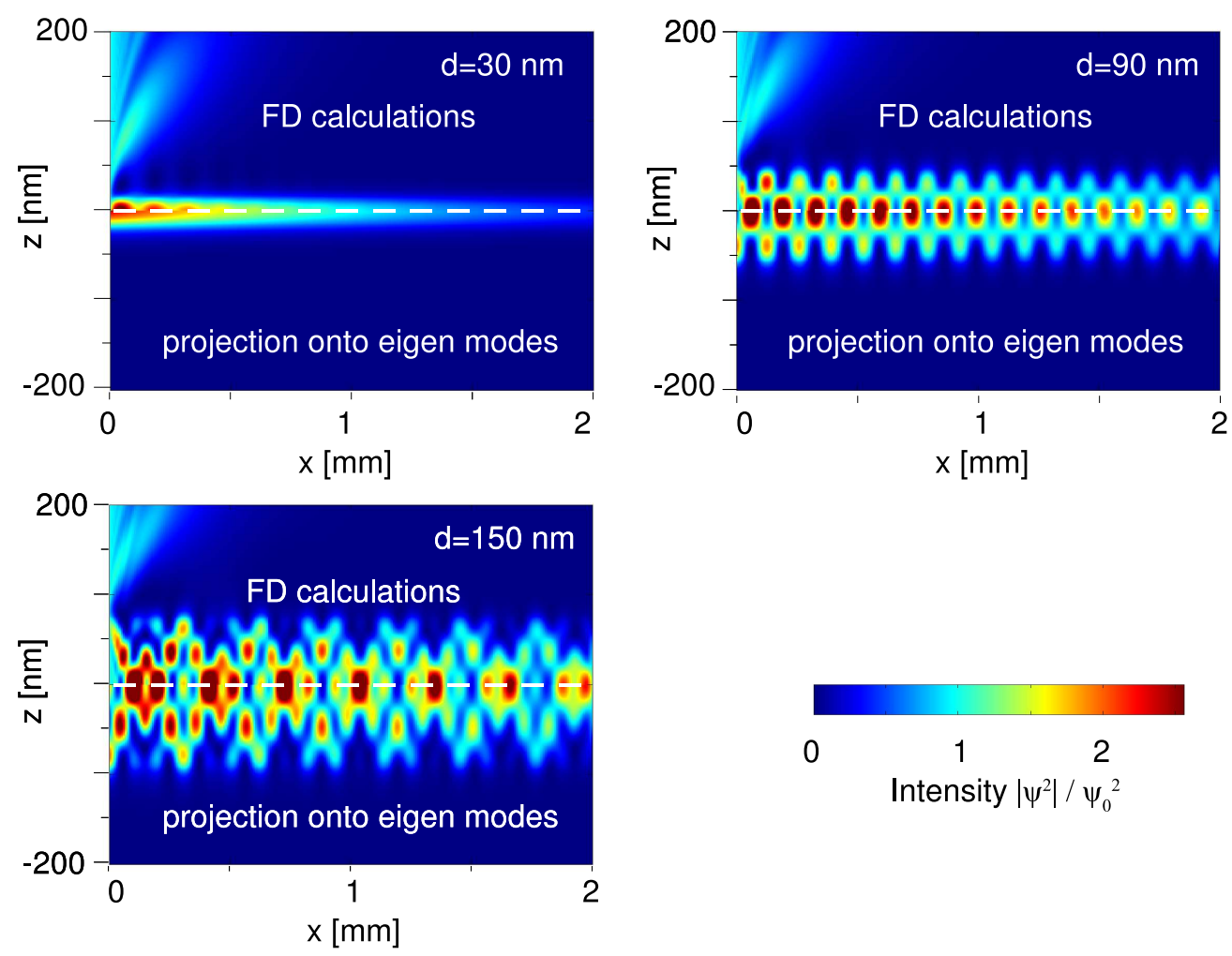

Figure 3.4: The electric field intensity inside planar waveguides calculated from both the finite difference (FD) approach (shown for $z>0$ ) and projecting the incident field onto the guided modes (shown for $z<0$ ). Far away from the entrance where the radiative modes visible in the FD calculations are damped out, the results are practically the same. The waveguides consist of a polyimide guiding layer $\left(\mathrm{C}_{22} \mathrm{H}_{10} \mathrm{~N}_{2} \mathrm{O}_{5}, \rho=1.42 \mathrm{~g} / \mathrm{cm}^{3}\right)$ in $\mathrm{Si}$, supporting one (d=30 $\mathrm{nm}$ ), three $(d=90 \mathrm{~nm})$, or five $(d=150 \mathrm{~nm})$ modes. Photon energy is $12.5 \mathrm{keV}$. 
( $d=90 \mathrm{~nm}$ and $d=150 \mathrm{~nm})$ multiple modes are excited, leading to a more complicated interference pattern due to the different propagation constants of the individual modes. From the field intensity at the end of the waveguide we conclude that transmission of the multi-mode waveguides is significantly higher than transmission of single-mode waveguides. This is due to the fact that in single-mode waveguides a large portion of power is propagating in the cladding, where absorption is much higher than in the guiding layer. This is studied in more detail in Section 4.2. However, a superposition of multiple modes will lower coherence of the exiting beam when the entrance of the waveguide is not illuminated coherently. A variation of the angle of incidence also strongly affects the field in the waveguide, as shown in the next section (Fig. 3.5) for an incidence angle of $0.03^{\circ}$. In this case, odd modes are also excited and the interference pattern is not symmetric any more.

\section{Determination of eigen modes}

Ignoring absorption, the electric field in a planar waveguide is given by

$$
\psi(x, z)=\sum_{m=0}^{N-1} c_{m} \psi_{m}(z) \exp \left(-i \beta_{m} x\right)
$$

Applying a Fourier transformation with respect to the propagation direction we find

$$
\left(\mathcal{F}_{x} \psi\right)\left(k_{x}, z\right)=\sum_{m=0}^{N-1} \sqrt{2 \pi} \delta_{D}\left(\beta_{m}-k_{x}\right) c_{m} \psi_{m}(z)
$$

where $\mathcal{F}_{x}$ denotes a Fourier transformation with respect to $x$ and $\delta_{D}$ denotes Dirac's delta function. Accordingly, this yields the eigen functions of the excited modes as well as the corresponding propagation constants. Since any real waveguide exhibits absorption at least for the evanescent wave in the cladding, the delta function is smeared out. However, a Fourier transform with respect to $x$ yields at least qualitatively the shape of the eigen functions as well as the propagation constants of the individual modes as shown in Fig. 3.5. Results of the calculations may thus be decomposed into contributions of individual guided modes.

\subsection{Two-dimensionally confining waveguides}

The numerical calculation of wave propagation in two-dimensionally confining waveguides requires the solution of the parabolic wave equation on a three-dimensional grid. Although the Crank-Nicolson scheme may in fact be extended to three dimensions, the resulting systems of linear equations are not tridiagonal any more and the algorithm becomes comparably slow [Tho95]. In three dimensions, two-step implicit alternating-direction schemes are much more efficient. Therefore, we develop an algorithm based on the PeacemanRachford scheme [PR55] that is second order accurate in $\Delta x, \Delta y$ and $\Delta z$ [Tho95]. 

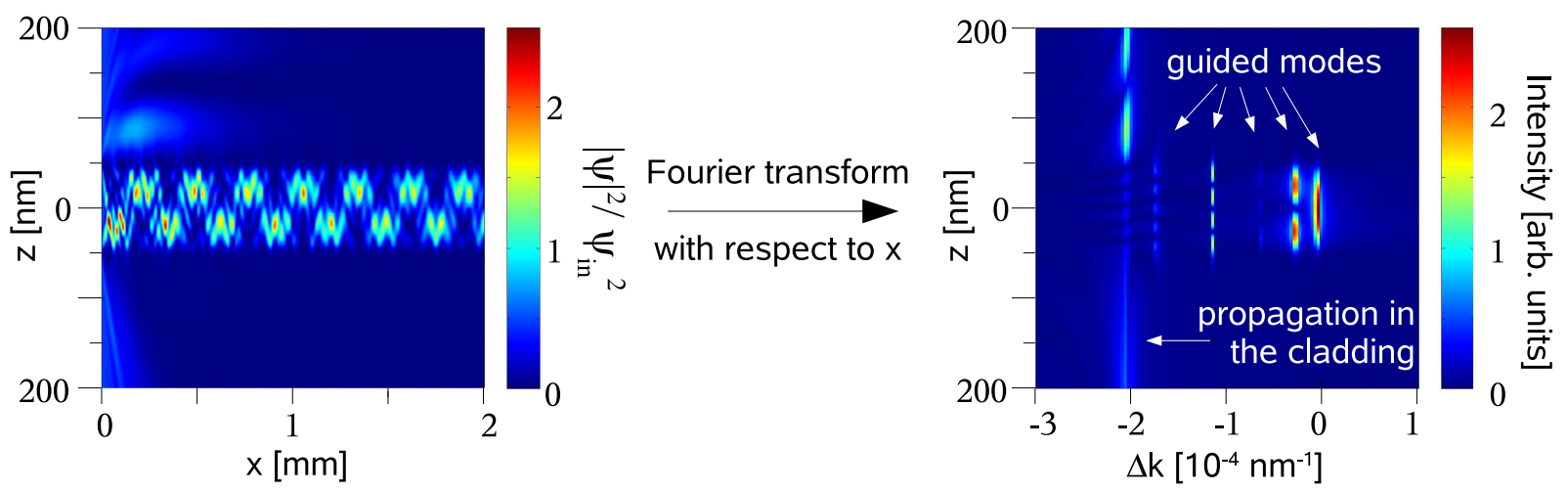

Figure 3.5: A Fourier transformation with respect to $x$ decomposes the electric field into the guided modes of the waveguide (90 nm vacuum gap in $S i$, angle of incidence $\alpha_{i}=0.03^{\circ}, E=$ $12.5 \mathrm{keV}) . \Delta k$ corresponds to the difference between the respective propagation constants $\beta_{m}$ and the wavenumber $k$ in free space. Note the standing wave above the guiding layer due to reflection of the beam from the guiding layer.

The algorithm consists of two different steps in which the partial derivatives with respect to $y$ and $z$ are alternately evaluated implicitly or explicitly. The increment in $x$ direction is $\Delta x / 2$ per step. In the first step, the parabolic wave equation is approximated by the finite-difference equation

$$
\frac{u_{j k}^{n+\frac{1}{2}}-u_{j k}^{n}}{\Delta x / 2}=\frac{A}{\Delta y^{2}} \delta_{y}^{2} u_{j k}^{n+\frac{1}{2}}+\frac{A}{\Delta z^{2}} \delta_{z}^{2} u_{j k}^{n}+\frac{1}{2}\left(F_{j k}^{n} u_{j k}^{n}+F_{j k}^{n+\frac{1}{2}} u_{j k}^{n+\frac{1}{2}}\right)
$$

with

$$
\begin{aligned}
\delta_{y}^{2} u_{j k}^{n} & :=u_{j-1 k}^{n}-2 u_{j k}^{n}+u_{j+1 k}^{n}, \\
\delta_{z}^{2} u_{j k}^{n} & :=u_{j k-1}^{n}-2 u_{j k}^{n}+u_{j k+1}^{n}, \\
F_{j k}^{n} & :=F\left(x_{n}, y_{j}, z_{k}\right) .
\end{aligned}
$$

Note that the partial derivative with respect to $y$ is evaluated implicitly, i. e. for $u_{j k}^{n+1 / 2}$, while the partial derivative with respect to $z$ is evaluated explicitly, i. e. for $u_{j k}^{n}$. With

$$
r_{y}:=\frac{A \Delta x}{\Delta y^{2}}, \quad r_{z}:=\frac{A \Delta x}{\Delta z^{2}}, \quad \text { and } \quad C_{j k}^{n}:=\frac{F_{j k}^{n} \Delta x}{4}
$$

equation (3.25) becomes

$$
\left(1-\frac{r_{y}}{2} \delta_{y}^{2}-C_{j k}^{n+\frac{1}{2}}\right) u_{j k}^{n+\frac{1}{2}}=\left(1+\frac{r_{z}}{2} \delta_{z}^{2}+C_{j k}^{n}\right) u_{j k}^{n}
$$


Taking into account the boundary conditions, we obtain the following system of linear equations

$$
\left(\begin{array}{ccccc}
B_{1}^{n} & \Theta & \ldots & & \\
\Theta & B_{2}^{n} & \Theta & \ldots & \\
& & \ddots & & \\
& \cdots & \Theta & B_{M_{z}-2}^{n} & \Theta \\
& & \cdots & \Theta & B_{M_{z}-1}^{n}
\end{array}\right)\left(\begin{array}{c}
\boldsymbol{u}_{1}^{n+\frac{1}{2}} \\
\boldsymbol{u}_{2}^{n+\frac{1}{2}} \\
\vdots \\
\boldsymbol{u}_{M_{z}-\frac{1}{2}}^{n+\frac{1}{2}} \\
\boldsymbol{u}_{M_{z}-1}^{n+\frac{1}{2}}
\end{array}\right)=\left(\begin{array}{c}
\boldsymbol{r}_{1} \\
\boldsymbol{r}_{2} \\
\vdots \\
\boldsymbol{r}_{M_{z}-2} \\
\boldsymbol{r}_{M_{z}-1}
\end{array}\right)
$$

with

$$
\begin{aligned}
& B_{k}^{n}=\left(\begin{array}{ccccc}
1+r_{y}-C_{1 k}^{n+\frac{1}{2}} & -\frac{r_{y}}{2} & 0 & \ldots & \\
-\frac{r_{y}}{2} & 1+r_{y}-C_{2 k}^{n+\frac{1}{2}} & -\frac{r_{y}}{2} & 0 & \ldots \\
\ldots & 0 & \ddots & & -\frac{r_{y}}{2} \\
& \cdots & -\frac{r_{y}}{2} & 1+r_{y}-C_{2 k}^{n+\frac{1}{2}} & 1+r_{y}-C_{M_{y}-1 k}^{n+\frac{1}{2}}
\end{array}\right), \\
& \boldsymbol{u}_{k}^{n+\frac{1}{2}}=\left(\begin{array}{c}
u_{1 k}^{n+\frac{1}{2}} \\
\vdots \\
u_{M_{y}-1, k}^{n+\frac{1}{2}}
\end{array}\right), \quad \text { and } \quad \boldsymbol{r}_{k}=\left(\begin{array}{c}
\left(1+\frac{r_{z}}{2} \delta_{z}^{2}+C_{1 k}^{n}\right) u_{1 k}^{n}+\frac{r_{y}}{2} u_{0 k}^{n+\frac{1}{2}} \\
\left(1+\frac{r_{z}}{2} \delta_{z}^{2}+C_{2 k}^{n}\right) u_{2 k}^{n} \\
\vdots \\
\left(1+\frac{r_{z}}{2} \delta_{z}^{2}+C_{M_{y}-2 k}^{n}\right) u_{M_{y}-2 k}^{n} \\
\left(1+\frac{r_{z}}{2} \delta_{z}^{2}+C_{M_{y}-1 k}^{n}\right) u_{M_{y}-1 k}^{n}+\frac{r_{y}}{2} u_{M_{y} k}^{n+\frac{1}{2}}
\end{array}\right)
\end{aligned}
$$

Here, $u_{0 k}^{n+\frac{1}{2}}$ and $u_{M_{y} k}^{n+\frac{1}{2}}$ are determined by the boundary condition given in equation 3.6 and $\Theta$ denotes the $\left(M_{y}-1\right) \times\left(M_{y}-1\right)$ zero matrix. Consequently, the first step consists of solving $M_{z}-1$ independent systems of linear equations:

$$
B_{k}^{n} \boldsymbol{u}_{k}^{n+\frac{1}{2}}=\boldsymbol{r}_{k}, \quad k=1, \ldots, M_{z}-1
$$

Since the $B_{k}^{n}$ are tridiagonal, each individual system can be solved within $\mathcal{O}\left(M_{y}\right)$ operations [Tho95] and the total time per step is in the order of $\mathcal{O}\left(M_{y} \times M_{z}\right)$. In the second step, we evaluate the partial derivatives with respect to $z$ implicitly and with respect to $y$ explicitly:

$$
\frac{u_{j k}^{n+1}-u_{j k}^{n+\frac{1}{2}}}{\Delta x / 2}=\frac{A}{\Delta y^{2}} \delta_{y}^{2} u_{j k}^{n+\frac{1}{2}}+\frac{A}{\Delta z^{2}} \delta_{z}^{2} u_{k}^{n+1}+\frac{1}{2}\left(F_{i j}^{n+\frac{1}{2}} u_{i j}^{n+\frac{1}{2}}+F_{i j}^{n+1} u_{i j}^{n+1}\right) .
$$

The solution to equation (3.35) is obtained analogously to that of equation 3.25). One has to solve $M_{y}-1$ independent systems of linear equations:

$$
B_{j}^{\prime n} \boldsymbol{u}_{j}^{\prime n+1}=\boldsymbol{r}_{j}^{\prime} \quad j=1, \ldots, M_{y}-1
$$


with

$$
\begin{aligned}
& B_{j}^{\prime n}=\left(\begin{array}{ccccc}
1+r_{z}-C_{j 1}^{n+1} & -\frac{r_{z}}{2} & 0 & \ldots & \\
-\frac{r_{z}}{2} & 1+r_{z}-C_{j 2}^{n+1} & -\frac{r_{z}}{2} & 0 & \ldots \\
& & \ddots & & \\
\cdots & 0 & -\frac{r_{z}}{2} & 1+r_{z}-C_{j 2}^{n+1} & -\frac{r_{z}}{2} \\
& \ldots & 0 & -\frac{r_{z}}{2} & 1+r_{z}-C_{j M_{z}-1}^{n+1}
\end{array}\right), \\
& \boldsymbol{u}_{j}^{\prime n+1}=\left(\begin{array}{c}
u_{j 1}^{n+1} \\
\vdots \\
u_{j M_{z}-1}^{n+1}
\end{array}\right), \quad \text { and } \quad \boldsymbol{r}_{j}^{\prime}=\left(\begin{array}{c}
\left(1+\frac{r_{y}}{2} \delta_{y}^{2}+C_{j 2}^{n+\frac{1}{2}}\right) u_{j 2}^{n+\frac{1}{2}} \\
\vdots \\
\left(1+\frac{r_{y}}{2} \delta_{y}^{2}+C_{j M_{z}-2}^{n+\frac{1}{2}}\right) u_{j M_{z}-2}^{n+\frac{1}{2}} \\
\left(1+\frac{r_{y}}{2} \delta_{y}^{2}+C_{j M_{z}-1}^{n+\frac{1}{2}}\right) u_{j M_{z}-1}^{n+\frac{1}{2}}+\frac{r_{z}}{2} u_{j M_{z}}^{n+1}
\end{array}\right) .
\end{aligned}
$$

Total time required to solve these systems of linear equations is in the order of $\mathcal{O}\left(M_{y} \times M_{z}\right)$. Accordingly, total time for each step is linear in both $M_{y}$ and $M_{z}$.

The algorithm was again implemented using the IDL programming language and the essential procedure of the source code is provided in the Appendix (Section A.4.2). Typical computational times on a Linux workstation with a $2.4 \mathrm{GHz}$ processor and $512 \mathrm{MB}$ RAM are several hours.

\subsubsection{Comparison to the weakly-guiding fibre}

To verify the finite-difference calculations in three dimensions, the field in cylindrical waveguides is calculated and compared to the analytical results given in Section 2.4.1 for weaklyguiding fibres. The incident wave is a plane wave at normal incidence. According to Eq. (2.39), the electric field in the fibre is given by a superposition of guided $\ell=0$ modes

$$
\psi(r, x)=\sum_{m} c_{m} \psi_{0 m}(r) \exp \left[\left(-i \beta_{m}-\mu_{m} / 2\right) x\right]
$$

with the eigen functions given by Eq. (2.34). The coefficients $c_{m}$ are given by the overlap integral

$$
c_{m}=2 \pi \int_{0}^{\infty} \psi_{0 m}(r) \psi_{\text {in }} r \mathrm{~d} r
$$

and $\beta_{m}=\sqrt{n_{1}^{2} k^{2}-u_{m}^{2} / a^{2}}$ are the propagation constants of the individual guided modes. $\mu_{m}$ is the linear attenuation coefficient weighted with respect to the intensity distribution. For the $m$-th mode it is given by

$$
\mu_{m}=2 \pi \int_{0}^{\infty}\left|\psi_{0 m}(r)\right|^{2} \mu(r) r \mathrm{~d} r .
$$




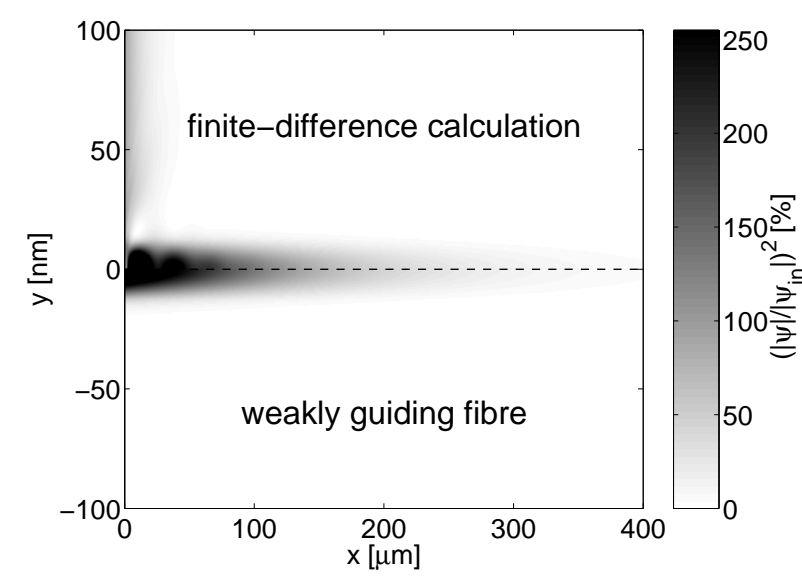

(a) $a=12 \mathrm{~nm}$.

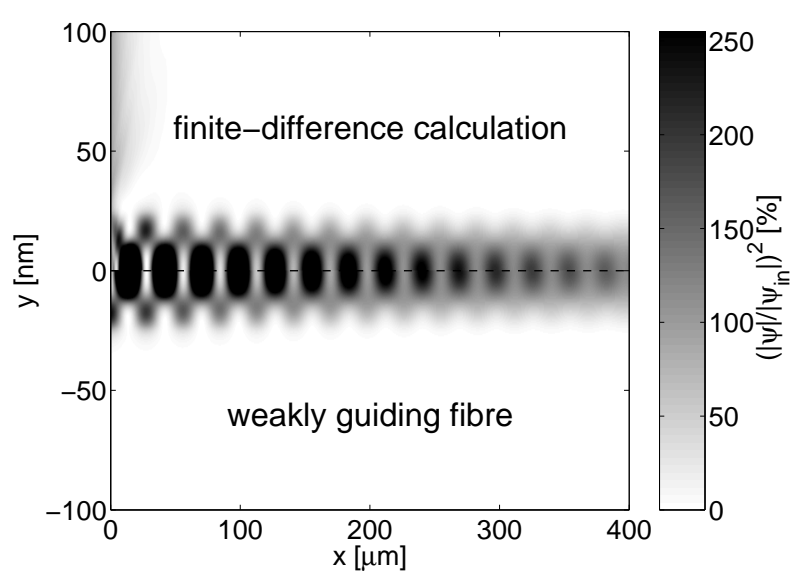

(c) $a=24 \mathrm{~nm}$.

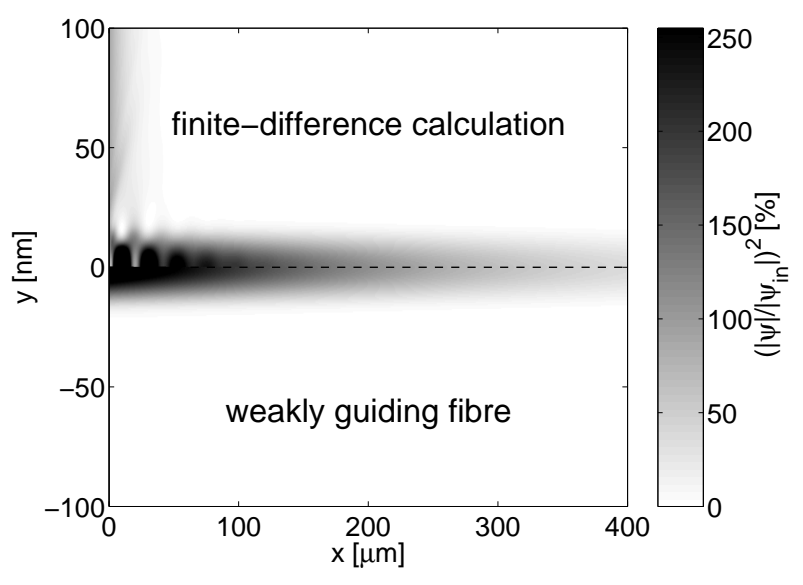

(b) $a=16 \mathrm{~nm}$.

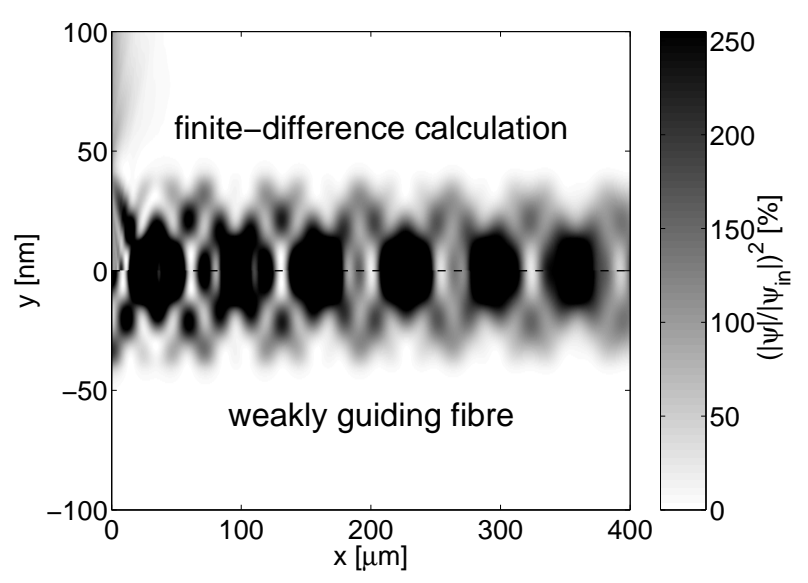

(d) $a=40 \mathrm{~nm}$.

Figure 3.6: Field intensities in cylindrical waveguides calculated with the finite-difference approach (shown for $y>0$ ) and the weakly-guiding-fibre approximation (shows for $y<0$ ) are practically the same. The fibres consist of a vacuum guiding core with a radius a between $12 \mathrm{~nm}$ (a) and $40 \mathrm{~nm}(\mathrm{~d})$ in Ge. Photon energy is $12.5 \mathrm{keV}$ and the number of excited modes increases from 1 to 3 .

The results of finite-difference calculations as well as analytical solutions obtained from the weakly-guiding fibre approximation are shown in Fig. 3.6. The fibre waveguides consist of a vacuum guiding core in Ge with radii ranging from $12 \mathrm{~nm}$ to $40 \mathrm{~nm}$. Far away from the entrance, both approaches yield practically identical results. We find the same modes propagating with the same propagation constants and subject to the same mode dependent absorption. Near the entrance, the finite-difference calculations also show how the over-illuminated radiation impinging on the cladding is absorbed. These contributions correspond to radiative modes ignored in the weakly-guiding fibre approximation. Therefore, these features cannot be observed in the field calculated by analytical theory. 


\subsection{Conclusions}

In conclusion, fast and adequate algorithms to solve the parabolic wave equation in two or three dimensions are presented. The results are verified by comparison to well-known analytical results. X-ray reflectivity calculated with these algorithms is in agreement with Fresnel reflectivity as long as the angle of incidence is in the regime of total external reflection. The results for planar and cylindrical waveguides are found in agreement with the respective analytical solutions for planar waveguides and weakly-guiding fibres.

One may thus expect the algorithms to yield reliable results even when analytical solutions are not available. This finally allows for comparably fast and easy field calculations applicable not only to a variety of one- and two-dimensionally confining waveguide structures, but to other X-ray optical devices as well [KPV95]. 


\section{Propagation of X-rays in ultra-narrow apertures}

Modern X-ray imaging, spectroscopy and lithography require the use of very small apertures with a cross-section far in the sub-micron range. The spatial resolution of these techniques is limited by the cross-sectional dimensions of the available apertures; see for example lens-less X-ray Fourier transform holography [ELS $\left.{ }^{+} 04\right]$ or proximity X-ray lithography $\left[K E L^{+} 04\right]$.

The fabrication of ultra-narrow apertures is a difficult task since, for hard $X$-rays in particular, large aspect ratios are required to ensure sufficient absorption in the cladding. Some possible realisations of ultra-small pinholes or slits are planar waveguides with a tunable air gap [ZvdVFN00], thin film structures [FJO+ 04], and pinholes fabricated using focused ion beams [ELS+ 04]. Apart from the practical problem of fabrication, there are also fundamental limitations for low diameters, namely the limit given by Bergemann et al. BKvdV03] (see Section 2.7).

In this chapter, the propagation of $X$-rays in very narrow slits or waveguides is studied. In particular, we address the question of how small an aperture can get until it has to be considered a waveguide. The onset of waveguiding effects is estimated analytically and quantified by the results of finite-difference field calculations (Section 4.1). A critical thickness is given below which waveguiding effects have to be taken into account. Then we consider the transmission of the smallest possible slits, i. e. planar single-mode waveguides, that may be significantly below one (Section 4.2).

For the sake of simplicity, we restrict ourselves to two-dimensional problems and study the transition from a slit into a slab waveguide. However, the treatment may straightforwardly be generalised to three dimensions and the same effects are expected for the transition from ultra-narrow pinholes to two-dimensionally confining waveguides.

\subsection{Onset of waveguiding effects in small apertures}

Large slits or pinholes can be described as apertures in an opaque screen. Apart from a phase shift due to the finite thickness, the electric field at the exit of the pinhole is practically the same as at the entrance, while nearly zero behind the cladding. Contrarily, for high aspect ratios we expect that the aperture cannot be taken into account by simple multiplication of a stepwise constant transmission function (diffraction aperture function), but that the transport of radiation is influenced by the mode characteristics known from the slab waveguide. 


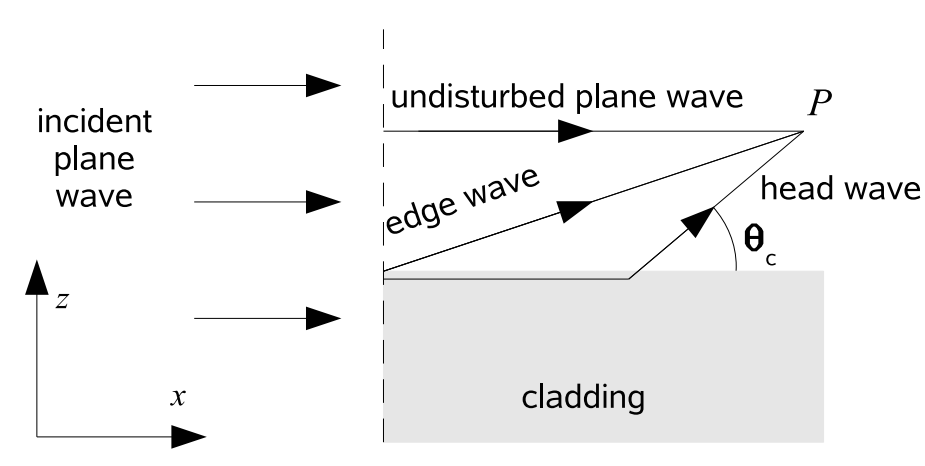

(a)

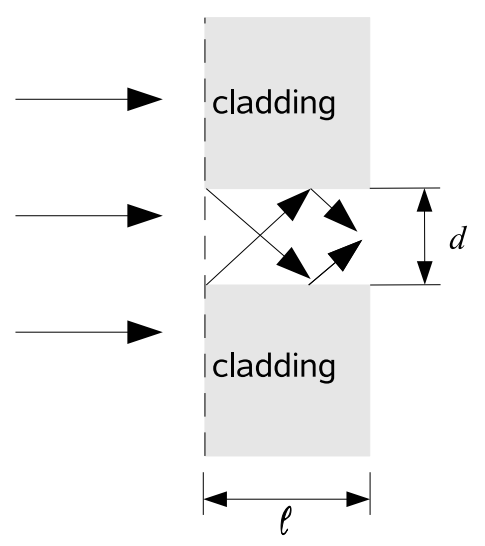

(b)

Figure 4.1: Diffraction at a dielectric wedge (a) and reflection of head waves in a waveguide (b).

Since the cladding around an aperture needs to be thick enough to provide sufficient absorption, high aspect ratios are necessary and one may expect waveguiding effects in very narrow apertures in a thick cladding. Hence, we expect significant differences from the approximation of a stepwise constant transmission function when $d$ becomes small.

In order to study the electric field in the aperture, we first consider diffraction of X-rays at a dielectric wedge, i. e. at the interfaces between the vacuum core and the cladding. This problem was studied in detail by Kopylov et al. [KPV96] within the approximation of the parabolic wave equation. An analytical solution of the parabolic wave equation was found showing that the electromagnetic field at an observation point $P$ in free space above the cladding may be understood as a superposition of three waves: (i) the undisturbed plane wave, (ii) an edge wave with the eikonal corresponding to the distance between $P$ and the edge, and (iii) a so-called head-wave propagating along the interface and exiting the cladding at the critical angle of total external reflection (see Fig. 4.1(a)). In an aperture, this simple picture is valid as long as the waves generated by the two interfaces towards the cladding do not interfere. In particular, if one of these waves hits the opposite interface at an angle less than $\theta_{c}$, reflection of these waves leads to the excitations of guided waves. One may then expect significant changes in the field at the exit of the aperture and in the far-field region compared to what one would expect behind an aperture in an opaque screen. Consequently, we expect the aperture approximation to fail when the head waves reach the opposite interfaces (Fig. 4.1(b)), i.e. when the diameter is below the critical width

$$
d_{c}=\ell \tan \theta_{c} \approx \ell \theta_{c}
$$

where $\ell$ denotes the length of the aperture. To illustrate this, the field inside slits surrounded by a material with $b / \delta=0.1$ was calculated within the dimensionless coordinates introduced in Section 2.8. This corresponds for instance to the optical properties of $\mathrm{Pt}$ at $E \approx 7.95 \mathrm{keV}$ with a critical width $W \approx 7.6 \mathrm{~nm}$. To ensure sufficient absorption, we 

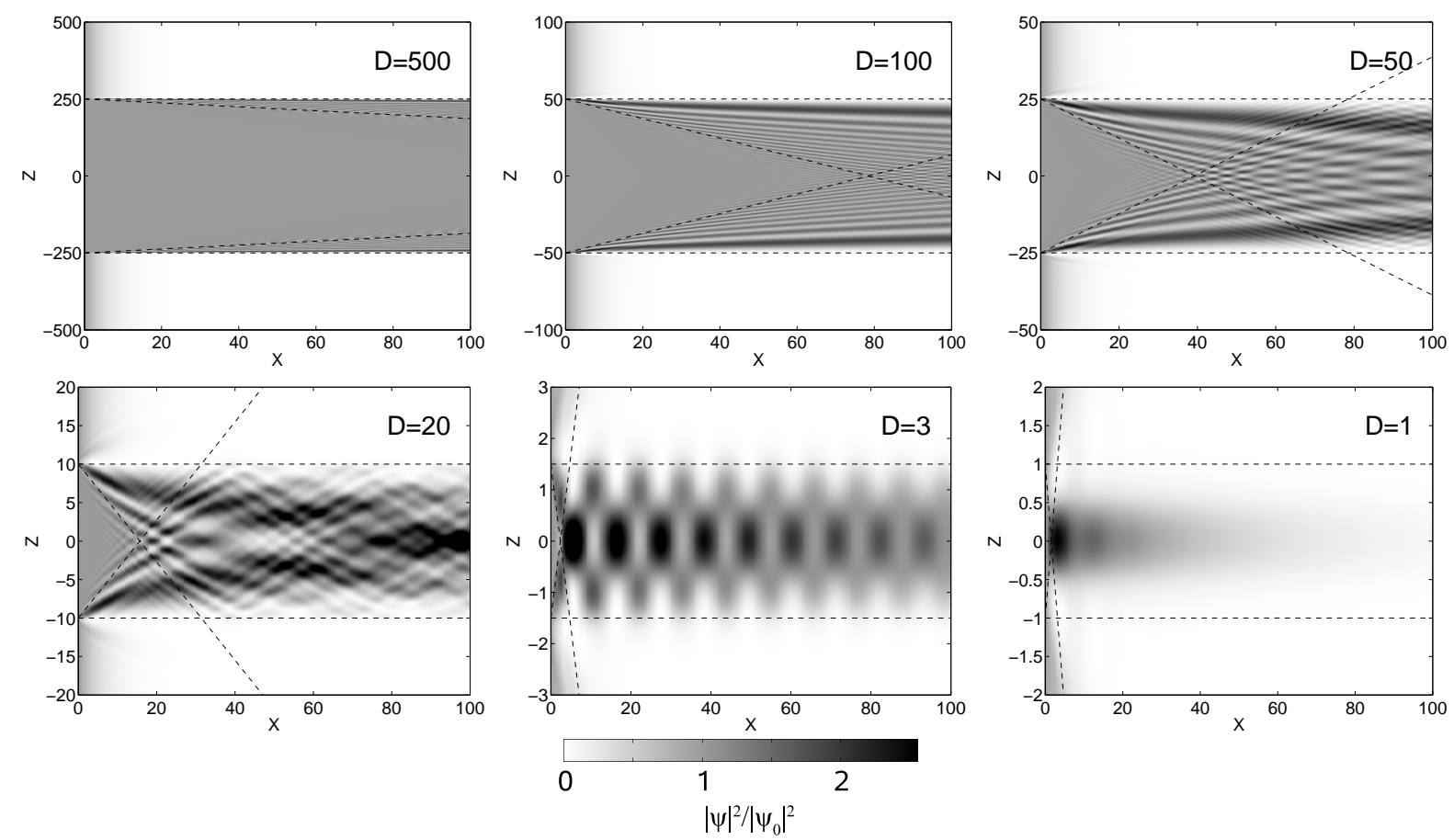

Figure 4.2: Electromagnetic field intensity in slits of different diameters $D=d / W$ for $b / \delta=0.1$. The dashed lines indicate the interfaces of the aperture and the critical angle of total external reflection, respectively. Dimensionless coordinates are used.

choose a length $\ell=100(k \delta)^{-1}$ corresponding to a transmission of $\exp (-20) \approx 2 \times 10^{-9}$ in the cladding. For Pt at $7.95 \mathrm{keV}$ this corresponds to a length of approximately $47 \mu \mathrm{m}$.

Fig. 4.2 shows the calculated field intensity inside slits of different dimensionless diameters $D:=d / W$. In the case of the largest diameter $(D=500)$, differences from a propagating plane wave are only visible near the interfaces and the head waves do not reach the opposite interfaces. With decreasing diameter, the head waves approach the opposite interfaces. When $d / \ell$ falls below the critical angle of total external reflection, these waves are reflected back into the slit and thus we have contributions of guided waves (Fig. 4.2, $D=50$ ). For further decreasing diameters, the field is more and more dominated by guided waves and we find the typical interference pattern of multi-mode waveguides. Finally, with $d$ approaching the critical width $W$, we have a single-mode waveguide and the field is given by the propagating fundamental mode (Fig. 4.2, $D=1$ ).

Fig. 4.3 shows the far-field diffraction patterns of slits with different diameters. The patterns are obtained as the squared modulus of the Fourier transforms of the calculated electromagnetic field at the exit of the slit. For better comparison, the spatial frequency $\nu$ is multiplied by the dimensionless slit diameter $D$ and the corresponding intensities $I(\nu)$ are divided by $D^{2}$. For $D=500$, the pattern is very close to a squared sinc function, i. e. the diffraction pattern of a slit in a flat opaque screen. For smaller diameters, waveguiding effects induce changes in the pattern and the exact shape becomes dependent on the length of the waveguide. For $D=2$ and $D=1$, we find the far-field diffraction pattern of a single- 


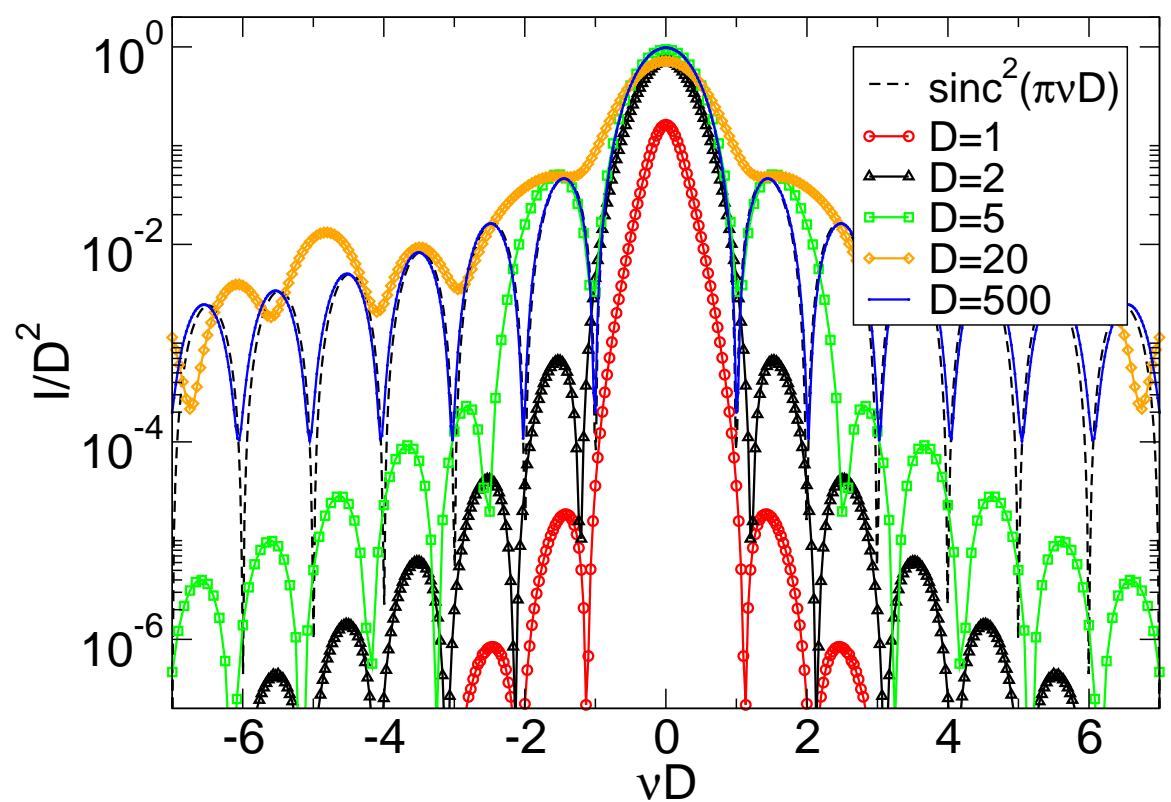

Figure 4.3: Transition to a waveguide observed in the far-field diffraction pattern which changes from a squared sinc function expected for an aperture in a flat opaque screen to that of a singlemode waveguide.

mode waveguide* Although we find nearly the same shape for $D=1$ and $D=2$, the intensity for $D=1$ is significantly lower, indicating strong absorption in the single-mode waveguide.

To investigate this in more detail, we calculated transmission of waveguides with diameters below $5 \mathrm{~W}$. We define the transmission of a waveguide as the ratio of incident intensity impinging on the guiding core to the total intensity exiting the waveguide. For small diameters, a significant amount of power propagates in the cladding and is therefore subject to strong absorption. This is clearly seen in Fig. 4.4, showing the calculated transmission of model waveguides with the parameters given above. The transmission of the single-mode waveguide with $d=W$ is only roughly $10 \%$ and for a diameter below half the critical width there is practically no transmission at all. However, these values depend critically on the length of the waveguide.

For some applications like proximity X-ray lithography [KEL ${ }^{+}$]], field intensity is of interest directly downstream of the aperture rather than in the far-field pattern. Fig. 4.5 shows the field intensity behind apertures of different size, and the evolution to the respective far-field diffraction pattern. Fig. 4.5(a) shows the field intensity downstream of a relatively large aperture $(D=100)$ turning into the Fraunhofer pattern given by a squared sinc function. The second calculation shows the field behind a multi-mode waveguide ( $D=10$, Fig. 4.5(b) turning into the corresponding Fraunhofer pattern of a multi-mode waveguide. In this regime, the exact shape of the pattern also depends on the length of the waveguide. If only one guided mode is excited ( $D=2$, Fig. 4.5(c) $)$, the shape of the exiting

${ }^{*}$ Note that for reasons of symmetry a plane wave at normal incidence only excites even modes. 


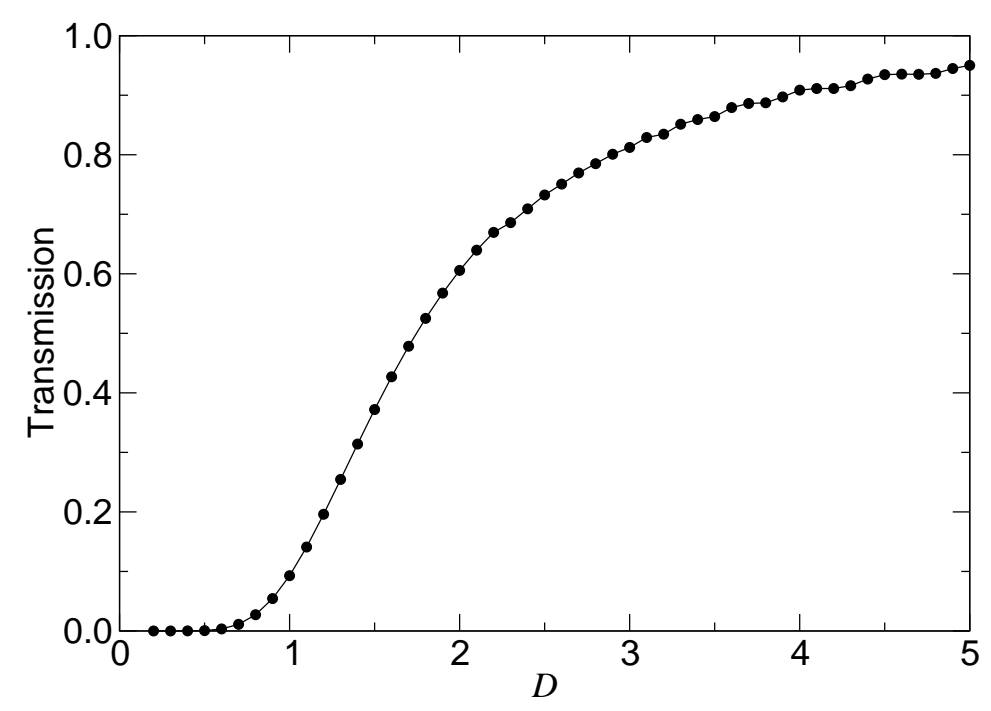

Figure 4.4: Transmission of very narrow waveguides decreases significantly for small diameters $D$ measured in units of the critical width $W$. Dimensionless coordinates are used and the parameters of the waveguide are $b / \delta=0.1$ and $\ell=100(k \delta)^{-1}$.

beam does not critically depend on the length of the waveguide. On a linear scale, the shape of the propagating fundamental mode is very similar to the corresponding far-field diffraction pattern, and the intensity pattern mainly broadens downstream of the waveguide. Note that for both the large aperture and the multi-mode waveguide, interferences lead to the formation of relatively small spots with an intensity exceeding the intensity of the incident plane wave. The full width at half maximum of these spots is significantly smaller than the aperture diameter. Similar effects have already been observed by Bergemann et al. [BKvdV03] close to the exit of tapered X-ray waveguides, but in the example shown here the small spot is located relatively far downstream of the waveguide. This effect could be very useful for scanning-probe applications or for bringing the resolution of proximity lithography significantly below the diameter of the aperture.

Far away from absorption edges, the dispersive part of the refractive index can be written as $\delta=\lambda^{2} r_{e} \rho_{e} / 2 \pi$. With $\theta_{c}=\sqrt{2 \delta}$ we find that the critical angle is inversely proportional to photon energy. Consequently, the critical diameter $d_{c}$ of a slit with a given length decreases with increasing photon energy. However, for reasonable experiments, the length of the aperture has to be long enough to ensure sufficient absorption in the cladding. Ignoring any absorption edges, the minimum length approximately scales with energy as $\ell \propto E^{3}$ for a given transmission of the cladding. In a rough approximation, the critical diameter $d_{c}$ for fixed cladding absorption thus scales with energy according to

$$
d_{c} \approx \theta_{c} \ell \propto E^{2}
$$

Accordingly, when the length is adapted to a given transmission of the cladding, the critical diameter below which waveguiding effects have to be taken into account increases with increasing photon energy. 


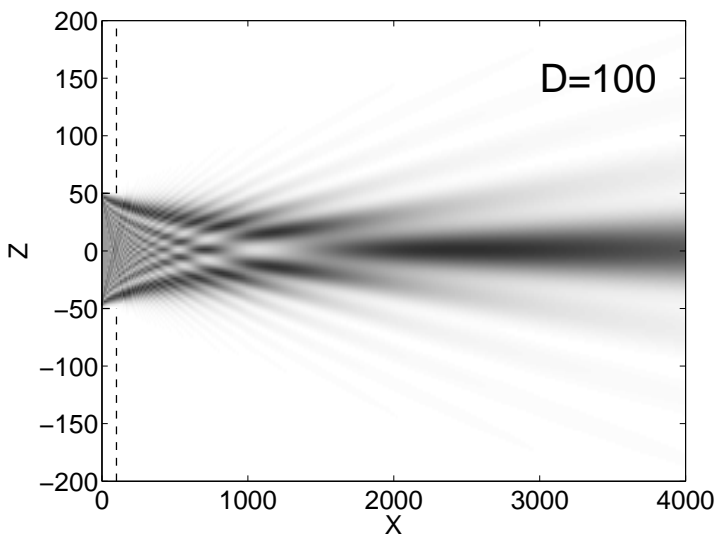

(a)

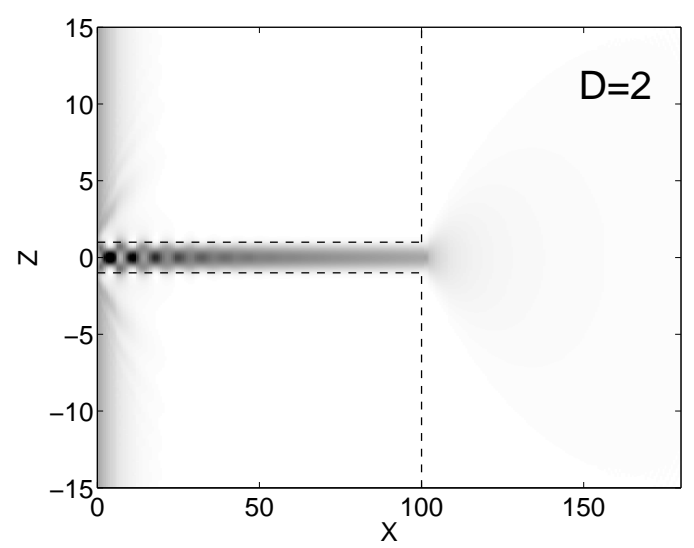

(c)

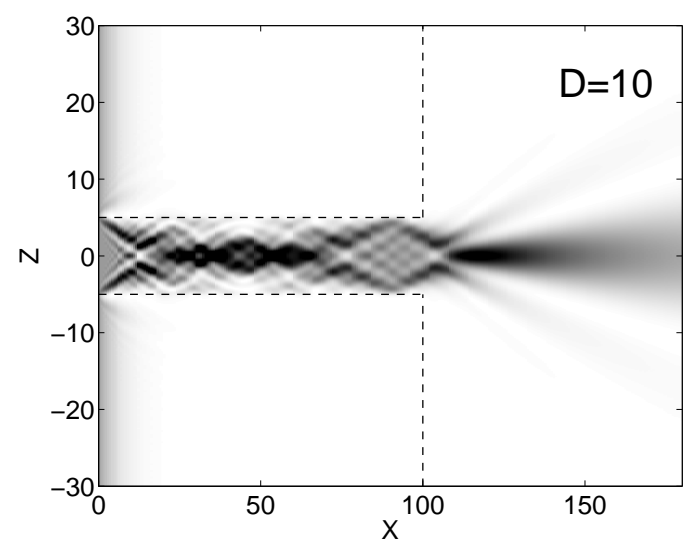

(b)

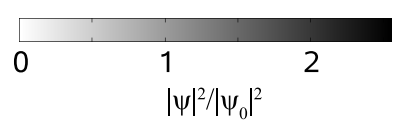

Figure 4.5: Finite-difference calculations illustrating how the field behind apertures of different diameters $D=d / W$ evolves into the respective far-field pattern. For large diameters $(D=100)$, the latter is given by a squared sinc function (a). Behind a small aperture with $D=10$, the field turns into the far-field diffraction pattern of a multi-mode waveguide (b) and the shape of the pattern depends also on the length of the waveguide. In contrast, the field behind a waveguide with $D=2 W$ becomes the typical length-independent far-field pattern of the fundamental mode (c). The dashed lines indicate the apertures. Dimensionless coordinates are used. 


\subsection{Transmission of single-mode waveguides}

Let us now consider the transmission of single-mode waveguides as a function of the waveguide length by analytical theory. More precisely, we consider the transmission of a waveguide with guiding core dimensions just at the limit between one and two guided modes. For a slab waveguide, according to equation (2.24), this limit corresponds to a waveguide parameter $V=\pi$. Sufficiently far away from the entrance, the electromagnetic field inside the single-mode waveguide is given by the propagating fundamental mode $\psi_{o}$ (see Section 2.3.2):

$$
\psi(x, z)=c_{0} \psi_{0}(z) \exp \left[\left(-i \beta_{0}-\mu_{0} / 2\right) x\right]
$$

with

$$
\psi_{0}(z)= \begin{cases}B_{0} \exp \left(-\gamma_{0} z\right), & \text { if } z \geq 0, \\ B_{0} \cos \kappa_{0} z+C_{0} \sin \kappa_{0} z, & \text { if } 0>z>-d, \\ \left(B_{0} \cos \kappa_{0} d+C_{0} \sin \kappa_{0} d\right) \times \exp \left[\gamma_{0}(z+d)\right], & \text { if } z \leq-d .\end{cases}
$$

For plane wave illumination $\psi_{\text {in }}=1$, the transmission of a waveguide with length $\ell$ is then given by ${ }^{*}$

$$
T(\ell)=\frac{\int\left|c_{0} \psi_{0}(z) \exp \left[\left(-i \beta_{0}-\mu_{0} / 2\right) \ell\right]\right|^{2} \mathrm{~d} z}{\int_{-d}^{0}\left|\psi_{\mathrm{in}}\right|^{2} \mathrm{~d} z}=\frac{\left|c_{0}\right|^{2} \exp \left(-\mu_{0} \ell\right)}{d} .
$$

The propagation constant $\beta_{0}$ as well as the constants $\kappa_{0}$ and $\gamma_{0}$ have to be determined numerically by solving the transcendental eigenvalue equation 2.22 . After extending the numerator and the denominator on the right-hand side of 2.22 with $d^{2}$, this equation reads

$$
\tan \left(\kappa_{0} d\right)=\frac{\left(2 \kappa_{0} d\right)\left(\gamma_{0} d\right)}{\left(\kappa_{0} d\right)^{2}-\left(\gamma_{0} d\right)^{2}}
$$

and has to be solved with the boundary condition (2.18) that may be written in the form

$$
\left(\gamma_{0} d\right)^{2}=V^{2}-\left(\kappa_{0} d\right)^{2}
$$

Evidently, the values of $\kappa_{0} d$ and $\gamma_{0} d$ are uniquely determined by $V$. For $V=\pi$ we find $\kappa_{0} d \approx 1.868$ and $\gamma_{0} d \approx 2.526$. Finally, we find $\left|c_{0}\right|^{2} \approx 1.979 d$ and the transmission is

$$
T \approx 1.979 \exp \left(-\mu_{0} \ell\right)
$$

where $\mu_{0}$ denotes the effective linear attenuation coefficient of the fundamental mode. In many experiments, the cross-sectional area of the incident beam is much larger than

*Within a strict mathematical consideration, this definition has the disadvantage that the numerator diverges in the case of an incident plane wave due to finite transmission of the cladding. However, in any experiment or finite-difference calculation the incident beam is confined to finite cross-section dimensions and the numerator is just the total flux downstream of the waveguide. In our analytical approach, we consider only the guided modes and the integral is finite as well. 
the entrance of the waveguide. Depending on the length of the waveguide and the overillumination at the front side of the waveguide, a significant amount of radiation may also be transmitted through the cladding, leading to unwanted background behind the aperture. Of course, this contribution also affects the far-field pattern. Consequently, the waveguide has to be long enough to ensure that the ratio $\sigma:=T / T_{\mathrm{cl}}$ of transmission $T$ in the waveguide and transmission $T_{\mathrm{cl}}$ in the cladding is high enough to achieve sufficient background suppression. The lowest tolerable $\sigma$ ratio depends on the cross-sectional dimensions of the incident beam and the waveguide as well as on the specific background requirements of the experiment. The corresponding length of the waveguide is then given as a function of $\sigma$ :

$$
\ell(\sigma)=\frac{\ln \sigma-\ln \left(\left|c_{0}\right|^{2} / d\right)}{\mu_{\mathrm{cl}}-\mu_{0}}
$$

where $\mu_{c l}$ is the linear attenuation coefficient of the cladding. The corresponding transmission is

$$
T(\sigma)=\left(\left|c_{0}\right|^{2} / d\right)^{1 /\left(1-\mu_{0} / \mu_{\mathrm{cl}}\right)} \sigma^{-\left(\mu_{0} / \mu_{\mathrm{cl}}\right) /\left(1-\mu_{0} / \mu_{\mathrm{cl}}\right)} .
$$

For a given $\sigma$, highest transmission is achieved when $\mu_{0} / \mu_{\mathrm{cl}}$ is minimal, i. e. in the case of a vacuum guiding layer. In this case we have $\mu_{0} / \mu_{\mathrm{cl}} \approx 0.156$ and

$$
T_{\mathrm{vac}}(\sigma) \approx 2.246 \sigma^{-0.185}
$$

The same treatment may be carried out for fibre waveguides. For a single-mode fibre with the critical radius $a_{c}$ given in Eq. 2.38), we find by an analogue treatment

$$
T_{\text {vac }}^{\mathrm{fibre}}(\sigma) \approx 3.436 \sigma^{-0.208} .
$$

The functions $T_{\text {vac }}(\sigma)$ and $T_{\text {vac }}^{\text {fibre }}(\sigma)$ are plotted in Fig. 4.6. For $\sigma$ less than $\approx 79$ transmission is greater than 1, i.e. the waveguide acts as a lens-like focusing device [Tie77]. Higher transmission of the fibre compared to the slab for low $\sigma$ is due to the fact that the fibre focuses the incident wave in two directions. On the other end, the stronger decay for high $\sigma$ is due to the fact that a larger portion of power is transmitted in the cladding, leading to stronger absorption of the propagating mode.

Let us close this section by discussing the maximum achievable transmission of waveguides with a cladding consisting of more than one material. In the following, it is shown that at least for some specific photon energies the transmission of single-mode waveguides may be enhanced by using two different materials in the cladding. As discussed above, absorption losses are mainly due to high intensity of the evanescent wave propagating in the cladding close to the guiding layer. A thin interlayer between the guiding core and the cladding, with optical constant $\delta$ close to the value in the cladding but with a significantly lower $b$, could decrease these losses without significantly changing the guided modes or transmission through the cladding.

Consider for example a single-mode planar vacuum waveguide in GaAs (Fig. 4.7(a)]. Let us suppose that GaAs is replaced by Ge within a range of $W / 2$ near the interfaces 


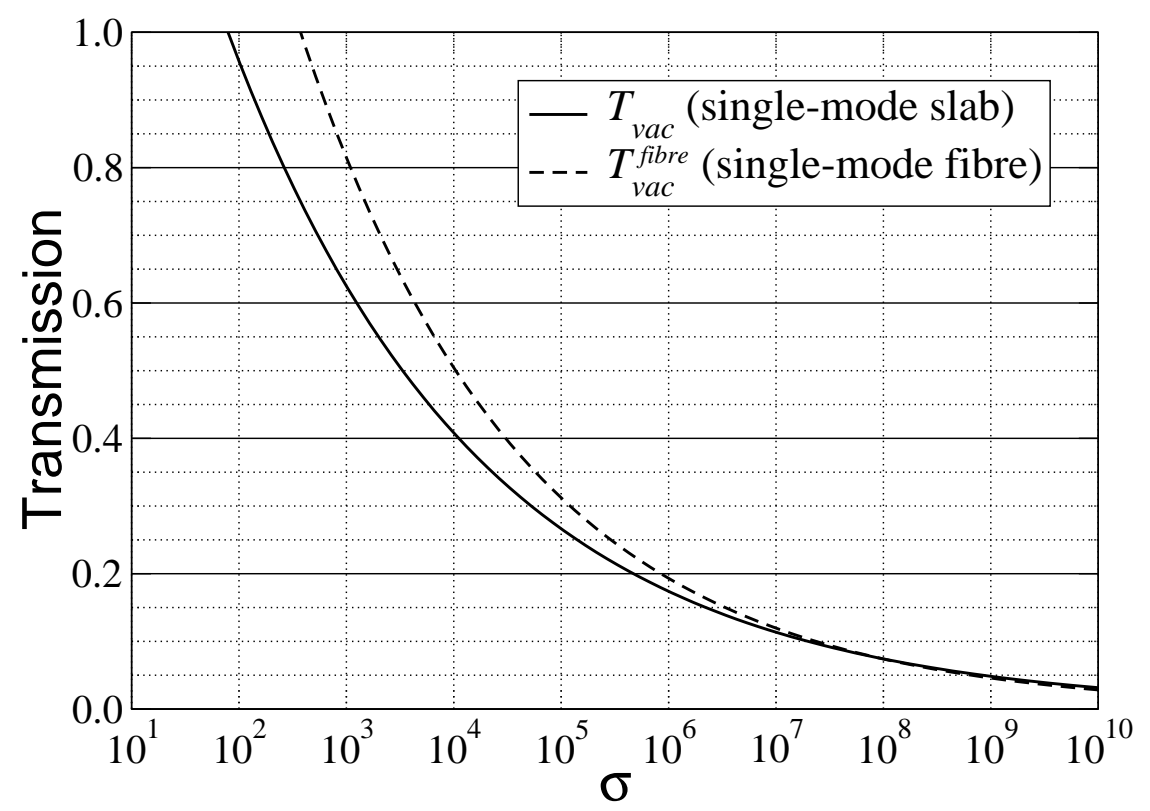

Figure 4.6: Transmission of single-mode waveguides with a vacuum guiding core calculated as a function of the ratio $\sigma:=T / T_{c l}$ to transmission $T_{c l}$ in the cladding. For high $\sigma$ values, transmission of the single-mode waveguides is significantly below 1 .

(Fig. 4.7(b) and photon energy is approximately $10.57 \mathrm{keV}$. At this energy (between the $\mathrm{K}$ absorption edges of $\mathrm{Ga}$ at $\approx 10.4 \mathrm{keV}$ and $\mathrm{Ge}$ at $\approx 11.1 \mathrm{keV}$ ) the real parts of the refractive indices of Ge and GaAs are practically the same [HGD93, but GaAs is much more absorbing. Thus, the thin Ge layer neither significantly changes the shape of the fundamental mode nor significantly decreases the absorption of over-illuminated radiation in the cladding. But a large portion of the evanescent wave propagating in the cladding is in this case located in Ge, where absorption is much weaker. Accordingly, the fundamental mode undergoes significantly reduced damping. The calculations shown in Fig. 4.7 are carried out for a length $\ell=150 W$. At $E=10.57 \mathrm{keV}$ we find $W=14.7 \mathrm{~nm}$. The $b / \delta$ ratios are 0.057 in the case of GaAs and 0.019 in the case of Ge, corresponding to a transmission of $\approx 1.8 \times 10^{-10}$ in GaAs. The presence of the Ge interlayer increases the transmission of the waveguide from less than $6 \%$ to more than $60 \%$. Obviously, for many other X-ray energies other favourable material combinations may be found. 


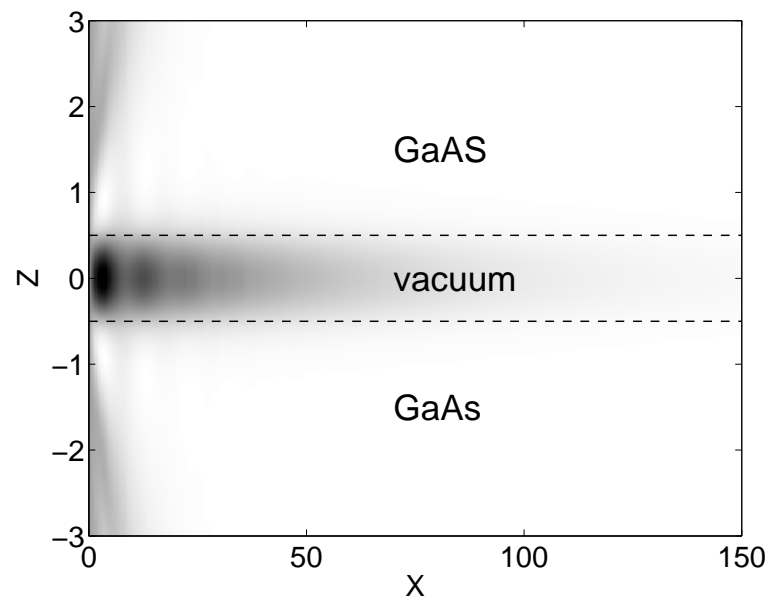

(a)

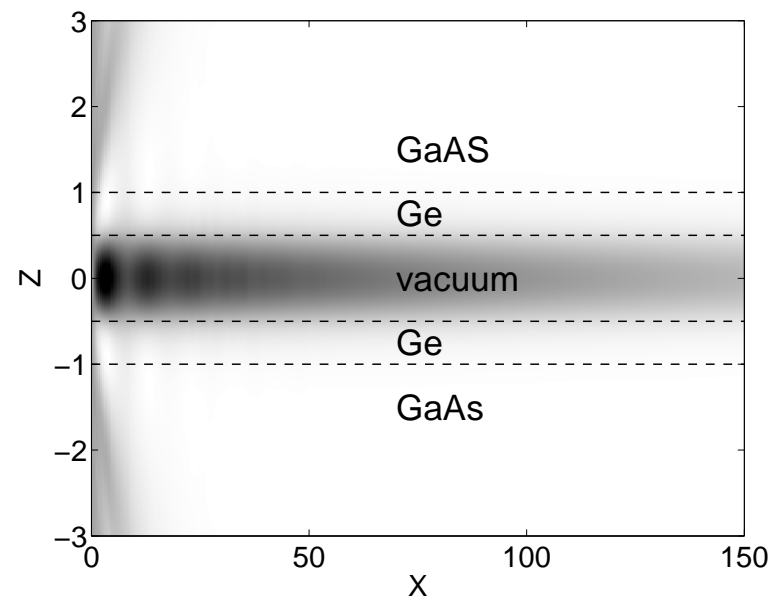

(b)

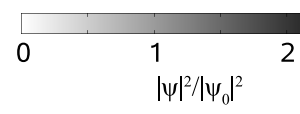

Figure 4.7: Transmission of a single-mode planar waveguide with a vacuum guiding core in GaAs at $E=10.57 \mathrm{keV}$ is strongly enhanced by adding a Ge interlayer of $0.5 \mathrm{~W}$ thickness. While the real part of the refractive index is practically the same for Ge and GaAs at this photon energy, lower absorption in the Ge interlayer leads to lower losses due to the evanescent wave propagating in the cladding. Dimensionless coordinates are used. 


\subsection{Conclusions}

We have studied the propagation of X-rays in ultra-narrow apertures and quantified a critical diameter $d_{c}$ below which waveguiding effects have to be taken into account. We have studied the changes in the field inside and directly behind an aperture as well as in the far-field region by finite-difference field calculations. It was shown how the shape of the transmitted wave and the total transmitted intensity changes with the aperture diameter. In conclusion, we may distinguish three different cases:

- As long as $d / \ell$ is large compared to the critical angle of total reflection, we may use the approximation of an aperture in a flat opaque screen. The electric field inside the slit is given by the incident plane wave except in a small region near the interfaces, where interferences with the head waves and edge waves are observed. The far-field diffraction pattern is well described by a squared sinc function.

- When $d / \ell$ falls below the critical angle, the head waves reach the opposite interfaces, where they are totally reflected. Thus, there are contributions of guided radiation. In this regime, the electric field in the slit is dominated by interference of the incident plane wave with the head waves and edge waves of both interfaces. The electric field at the exit of the waveguide as well as the far-field diffraction pattern depend critically on the length and diameter of the waveguide. With further decreasing diameter, the electric field approaches the field given by the superposition of guided modes as expected for a planar waveguide.

- For $d \approx W$, the electric field in the waveguide is dominated by the fundamental guided mode. Variations of the waveguide length change the intensity of the far-field diffraction pattern but not its shape. The transmission of the aperture decreases strongly with decreasing diameter.

In particular, transmission of single-mode waveguides may be significantly smaller than 1 , depending on the required absorption of over-illuminated radiation in the cladding. However, it may be strongly enhanced by relaxing the strict demand for only one guided mode or by fabrication of single-mode waveguides with an appropriate interlayer between the cladding and the guiding core. 


\section{Waveguide-based imaging}

This chapter introduces waveguide-based imaging as lensless X-ray projection microscopy. The waveguide is considered as a point-like source and a magnified image of the sample is projected onto the detector. For large objects this simply yields a magnified absorption image known from classical $X$-ray radiography. Contrarily, the image recorded for a small object is given rather by its Fresnel diffraction pattern, since the waveguide acts as a small coherent source.

The experiment setup corresponds to Gabor's "microscopic principle" invented in 1948 Gab48, Gab49] as lensless electron projection microscopy, today known as in-line holography. Motivated by the lack of X-ray lenses, Baez suggested in 1952 the application of Gabor's principle to X-rays [Bae52]. Others, like El-Sum and Kirkpatrick, used visible light to reconstruct in-line holograms recorded with point-focus $X$-ray tubes on photographic films [ESK52, ES52]. However, Baez found the obtainable resolution limited by either the resolving power of the film or the size of the available incoherent X-ray sources, and concluded that high resolution is easier achieved using visible light [Bae52].

With the availability of synchrotron radiation and new recording media, and with the possibility of computational reconstruction, interest in X-ray holography was revived in the 1980s [How84]. However, using a parallel beam, the spatial resolution was still limited to the spatial resolution of the detector. In the late 1980s, a resolution of 40-50 nm could be demonstrated using soft X-rays and a polymer photo-resist as detector [HJK ${ }^{+} 87$ ]. The in-line holograms were recorded in a parallel beam, read out using electron microscopy [JHKR90] or atomic-force microscopy [LHJK96], and finally reconstructed numerically.

To overcome the limitations implied by comparably low resolution detectors, Fourier transform holography [MKJ ${ }^{+}$22, ELE $E^{+} 04$, ELS $\left.{ }^{+} 04\right]$ was demonstrated using X-rays. In 1997, Watanabe et al. used a back-illuminated CCD detector and illuminated a $1 \mu \mathrm{m}$ pinhole with a zone plate. The pinhole thus acted as a small incoherent source and a magnified in-line hologram was recorded using a CCD detector [WSTA97]. Although resolution was only $1 \mu \mathrm{m}$ on $578 \times 385$ pixels and typical exposure time was 15-30 minutes, they already pointed out the potential of higher resolution and real-time observation with smaller sources providing more intense beams.

Waveguide-based holographic imaging offers the possibility to achieve spatial resolution down to the cross-sectional dimensions of the waveguide. In combination with a highly efficient CCD camera, a comparably low dose and nearly real-time reconstruction is possible. For hard X-rays, the interaction with the sample is mainly phase shift. Accordingly, only a low dose is absorbed and one may expect radiation damage to be low as well. 


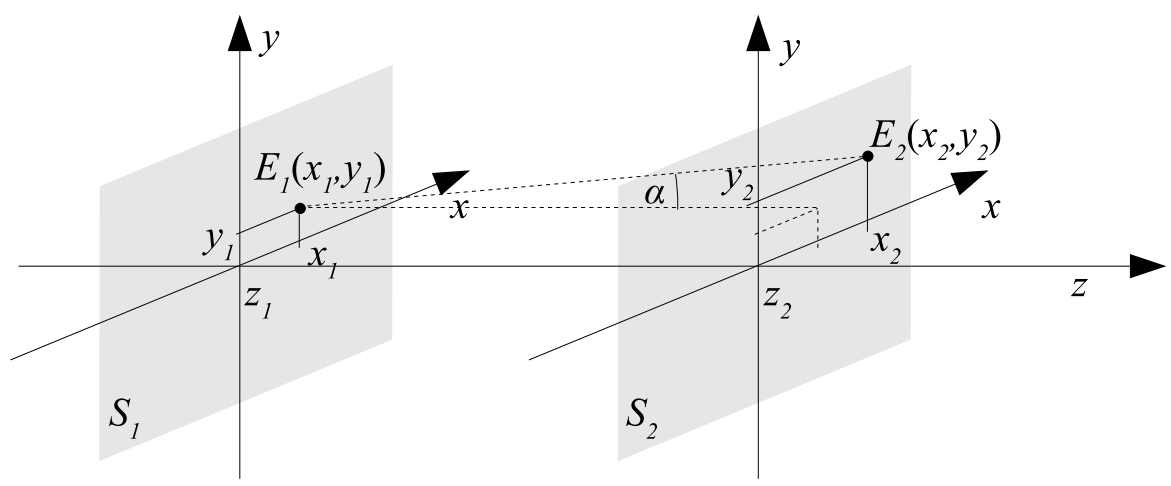

Figure 5.1: The wave field $E_{2}$ in plane $S_{2}$ can be determined from a known wave field $E_{1}$ in plane $S_{1}$ by applying the Huygens-Fresnel principle.

In this chapter, the imaging process is described based on scalar diffraction theory (Section 5.1). A sample is described by its optical transmission function and we consider image contrast in terms of contrast transfer functions. We point out different imaging regimes and give a brief introduction to holography (Section 5.2). It is shown that waveguide-based imaging may be interpreted as point-source in-line holography ("Gabor microscopy"). We quantify the corresponding spatial resolution and point out the limitations of in-line holography, namely the generation of an inseparable twin-image severely disturbing image reconstruction. Off-axis holography is introduced to overcome the twin-image problem, and it is show how a magnified off-axis hologram can be recorded with a two-beam Gabor microscope. Finally, we account for practical aspects like detector resolution, the field of view, and noise originating from a limited number of photons (Section 5.3).

Note that the orientation of the coordinate system is changed with respect to the previous chapters. In the previous chapters, the choice of the coordinate system is motivated by the axis denotation at synchrotron beamlines where $z$ usually denotes the vertical direction and the beam is propagating in a horizontal direction. However, in textbooks about optics [BW87, Hec89, LK03, LLT97], z denotes the optical axis. Therefore we now change the notation. To avoid confusion, the scalar electric field is now denoted by the symbol $E$ indicating the changed coordinate system.

\subsection{Scalar diffraction theory}

This section describes the propagation of X-rays downstream of the waveguide. We restrict ourselves to the case of coherent monochromatic radiation. Polychromatic radiation may then be treated as an incoherent superposition of monochromatic waves.

According to the Huygens-Fresnel principle, the wave field $E_{2}$ in a plane $S_{2}$ is given as a superposition of the amplitudes of spherical wavelets originating from each point in plane $S_{1}$ (see Fig. 5.1). The mathematical formulation is known as the Fresnel-Kirchhoff diffraction formula [BW87, LK03]: 


$$
E_{2}\left(x_{2}, y_{2}\right)=\frac{1}{i \lambda} \int E_{1}\left(x_{1}, y_{1}\right) \frac{e^{i k r}}{r} \cos \alpha \mathrm{d} x_{1} \mathrm{~d} y_{1}
$$

with

$$
r=\sqrt{\left(x_{2}-x_{1}\right)^{2}+\left(y_{2}-y_{1}\right)^{2}+\left(z_{2}-z_{1}\right)^{2}} .
$$

In the following, the directional factor $\cos \alpha$ is ignored since $\alpha$ is in the order of milliradians in hard X-ray imaging. Furthermore, $x$ and $y$ are usually small compared to the distance $z:=z_{2}-z_{1}$. We may approximate $1 / r$ in Eq. (5.1) by $1 / z$ and replace $r$ in the exponential term by

$$
r \approx z+\frac{\left(x_{2}-x_{1}\right)^{2}+\left(y_{2}-y_{1}\right)^{2}}{2 z} .
$$

This is known as the paraxial approximation (sometimes also called Fresnel approximation) and the Fresnel-Kirchhoff formula reads

$$
E_{2}\left(x_{2}, y_{2}\right)=\frac{\exp (i k z)}{i \lambda z} \int E_{1}\left(x_{1}, y_{1}\right) \exp \left(i k \frac{\left(x_{2}-x_{1}\right)^{2}+\left(y_{2}-y_{1}\right)^{2}}{2 z}\right) \mathrm{d} x_{1} \mathrm{~d} y_{1} .
$$

Introducing the propagator kernel [LK03, Wei02]

$$
h_{z}(x, y):=\frac{\exp (i k z)}{i \lambda z} \exp \left(\frac{i k}{2 z}\left(x^{2}+y^{2}\right)\right)
$$

we may write the Fresnel-Kirchhoff integral (5.4) as a convolution of the field $E_{1}\left(x_{1}, y_{1}\right)$ with the propagator kernel $h_{z}$ :

$$
E_{2}\left(x_{2}, y_{2}\right)=\int E_{1}\left(x_{1}, y_{1}\right) h_{z}\left(x_{2}-x_{1}, y_{2}-y_{1}\right) \mathrm{d} x_{1} \mathrm{~d} y_{1}
$$

This convolution in real space corresponds to a multiplication in Fourier space (see Eq. A.5)):

$$
\tilde{E}_{2}=\tilde{h}_{z} \times \tilde{E}_{1}
$$

The tilde denotes the Fourier transform. In the paraxial approximation we find

$$
\tilde{h}_{z}\left(\nu_{x}, \nu_{y}\right)=-\exp (i k z) \times \exp \left[-i \pi \lambda z\left(\nu_{x}^{2}+\nu_{y}^{2}\right)\right]
$$

where $\nu_{x}$ and $\nu_{y}$ denote the respective spatial frequencies. The first term is just a simple phase term while the exponential term $\exp \left[-i \pi \lambda z\left(\nu_{x}^{2}+\nu_{y}^{2}\right)\right]$ is also known as the optical transfer function for Fresnel diffraction [PGW97.

A straightforward numerical evaluation of the Fresnel-Kirchhoff integral in real space is very time-consuming. For $N \times N$ pixels in plane $S_{1}$, the calculation of the electric field at a point $\left(x_{2}, y_{2}\right)$ in plane $S_{2}$ requires an integration over $N \times N$ pixels and the corresponding time scales with $N^{2}$. Assuming $N \times N$ pixels in plane $S_{2}$ as well, the total time scales with $N^{4}$. In practice, this means that the propagation of a field with $1024 \times 1024$ pixels may take a few hours on a standard PC.

Therefore, propagation is carried out in Fourier space based on Eq. (5.7). The twodimensional Fourier transformation may be carried out within $\mathcal{O}\left(N^{2} \log _{2} N\right)$ operations by 
a "Fast Fourier transformation" [Pre92]. The multiplication of $\tilde{h}_{z}$ and $\tilde{E}_{1}$ takes only $N^{2}$ operations and the complete calculation may thus be carried out by $\mathcal{O}\left(N^{2} \log _{2} N+N^{2}\right)$ operations. For $N=1024$, this corresponds to a total time of about a second on a standard PC. The source code of a relevant IDL program is provided in the Appendix A.4.3).

Note that the paraxial approximation of the Fresnel-Kirchhoff integral is equivalent to the parabolic wave equation with $n=1$ (see Section A.3). Thus, the same results could in principle be obtained from the finite-difference calculations presented in Chapter 3 . However, the latter would be much more time-consuming.

\subsubsection{The optical transmission function}

When X-rays propagate through a uniform slab of thickness $t$, the resulting phase change compared to a beam travelling in vacuum is

$$
\phi=-k \delta t
$$

Ignoring generally small dispersion corrections, we may use Eq. (2.3) and find

$$
\phi=-\lambda \rho_{e} r_{e} t
$$

Accordingly, the phase shift measures the projected electron density. At the same time, intensity of the beam decreases by a factor $\exp \left(-\mu_{a} t\right)$ where $\mu_{a}$ is the linear attenuation coefficient. For a more general sample, $\phi$ and $\mu:=\mu_{a} t$ depend on the coordinates $(x, y)$. The object then changes complex amplitude of the wave field by

$$
f(x, y)=\exp (i k t) \times \underbrace{\exp [i \phi(x, y)-\mu(x, y) / 2]}_{=\tau(x, y)}
$$

where the second term is called the optical transmission function $\tau(x, y)$ of the object.

\subsubsection{Contrast transfer function for weak objects}

We now consider a thin object illuminated by a plane wave. In an appropriately chosen coordinate system, the wave field behind the object is given by its optical transmission function $\tau(x, y)$. In the case of weak objects where $\phi$ and $\mu$ are small compared to 1 , we may expand the optical transmission function given in Eq. (5.11):

$$
\tau(x, y)=\exp [i \phi(x, y)-\mu(x, y) / 2] \approx 1+i \phi(x, y)-\mu(x, y) / 2 .
$$

Applying Eq. (5.7) and Eq. (5.8), we find for the intensity in plane $S_{2}$ further downstream in Fourier space

$$
\begin{aligned}
\tilde{E}_{2}\left(\nu_{x}, \nu_{y}\right) & =\tilde{\tau} \times \tilde{h}_{z} \\
& \approx\left(\delta_{D}\left(\nu_{x}, \nu_{y}\right)+\tilde{\phi}\left(\nu_{x}, \nu_{y}\right)-\tilde{\mu}\left(\nu_{x}, \nu_{y}\right) / 2\right) \times \tilde{h}_{z} \\
& =-\left(\delta_{D}\left(\nu_{x}, \nu_{y}\right)+\tilde{\phi}\left(\nu_{x}, \nu_{y}\right)-\tilde{\mu}\left(\nu_{x}, \nu_{y}\right) / 2\right) \exp (i k z) \times \exp \left[-i \pi \lambda z\left(\nu_{x}{ }^{2}+\nu_{y}{ }^{2}\right)\right]
\end{aligned}
$$




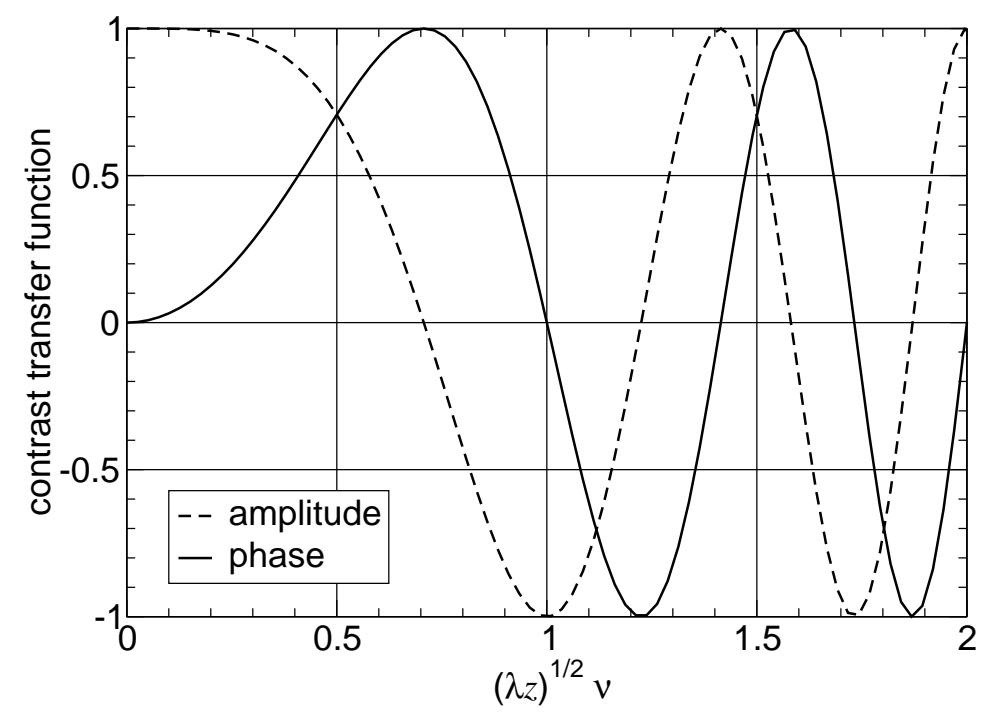

Figure 5.2: Contrast transfer functions for phase and amplitude objects as a function of the normalised spatial frequency $\sqrt{\lambda z} \nu$.

where $\delta_{D}$ denotes the Dirac delta function representing the directly transmitted beam. To first order in $\phi$ and $\mu$ the corresponding intensity $I=\left|E_{2}\right|^{2}$ is given in Fourier space by $\mathrm{MMW}^{+} 02$

$$
\tilde{I}\left(\nu_{x}, \nu_{y}\right) \approx \delta_{D}\left(\nu_{x}, \nu_{y}\right)+2 \tilde{\phi}\left(\nu_{x}, \nu_{y}\right) \sin \chi-\tilde{\mu}\left(\nu_{x}, \nu_{y}\right) \cos \chi
$$

with $\chi=\pi \lambda z\left(\nu_{x}^{2}+\nu_{y}^{2}\right)$. The terms $\sin \chi$ and $\cos \chi$ are called phase and amplitude contrast transfer functions (CTFs) of the Fresnel diffraction pattern. Fig. 5.2 shows the one-dimensional CTFs as a function of the normalised frequency $\sqrt{\lambda z} \nu$. Thus, the real and imaginary parts of the CTF may be interpreted in terms of image contrast of the amplitude and phase components of the object transmission function. We now distinguish the following imaging regimes:

Contact image: Directly behind the object $(z=0)$ the intensity distribution shows pure and maximum amplitude contrast and phase contrast is zero.

"Direct-contrast" regime: As long as $\chi$ is small compared to $\pi / 2$, amplitude contrast decreases with increasing distance and phase contrast increases. With $\sin \chi \approx \chi$ the intensity behind a pure phase object is $\mathrm{MMW}^{+} 02$

$$
\tilde{I}\left(\nu_{x}, \nu_{y}\right)=\delta_{D}(u, v)+2 \pi \lambda z\left(\nu_{x}^{2}+\nu_{y}^{2}\right) \tilde{\phi}\left(\nu_{x}, \nu_{y}\right) .
$$

Accordingly, intensity in real space is proportional to the two-dimensional Laplacian $\nabla_{\perp}^{2}$ of the phase shift and to the distance $z$ (see Eq. A.3p):

$$
I(x, y)=1-\frac{\lambda z}{2 \pi} \nabla_{\perp}^{2} \phi(x, y)
$$

* Note that this corresponds to a normalisation with respect to the radius of the first Fresnel zone Clo99. 


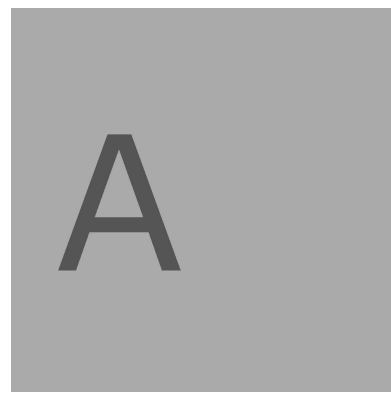

(a) $z=0$.

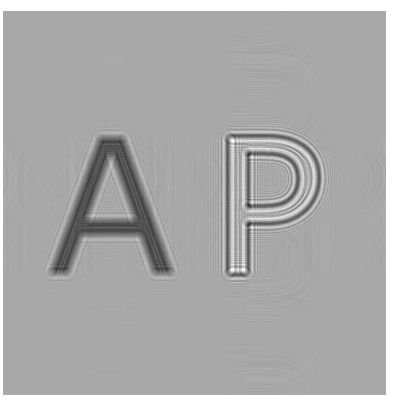

(b) $z=0.2 \mathrm{~mm}$.

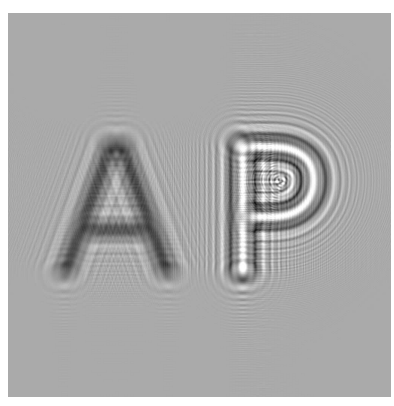

(c) $z=1 \mathrm{~mm}$.

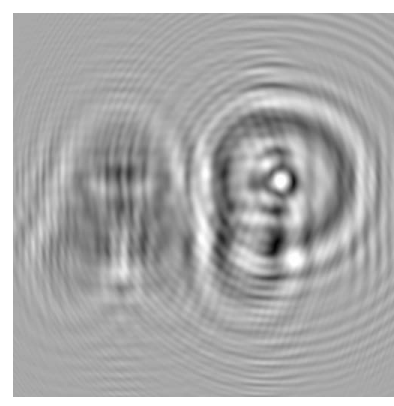

(d) $z=10 \mathrm{~mm}$.

Figure 5.3: Intensity in the different imaging regimes behind a pure amplitude object (" $A$ ", $50 \%$ transmission) and a pure phase object ("P", phase shift $\pi / 2$ ) illuminated by a plane wave. The wavelength is $0.1 \mathrm{~nm}$ and the size of the images is $10 \times 10 \mu \mathrm{m}^{2}$. In the contact image (a) only the amplitude object is visible. With increasing distance $z$ the edges of the phase object get visible (b). With further increasing distance the interference fringes become larger (c) and direct resemblance to the object gets more and more lost in the holographic regime (d).

Note that within this regime the X-ray wavelength only scales the image contrast. Accordingly, high monochromacity is not required and a broad bandwidth may be used.

Holographic regime: With further increasing distance $z$, the absolute value as well as the sign of the image contrast depend crucially on the spatial frequency and the Xray wavelength. Consequently, both amplitude and phase images loose direct resemblance to the object. Comparably high monochromacity is required and holographic reconstruction is necessary (see Section 5.2.

The different imaging regimes are illustrated in Fig. 5.3. At short distances, the decrease of the phase CTF limits the accessibility to low frequencies for phase objects. Accordingly, only the edges of the object are visible. However, since the image of an object contains a whole range of spatial frequencies, large features (corresponding to small spatial frequencies) may be in the direct-contrast regime, while small features (corresponding to high spatial frequencies) are already in the holographic regime. This is the typical situation of the experiments presented in Chapter 7 .

\subsubsection{Validity of the Fresnel approximation}

To verify the validity of the Fresnel approximation (5.3), we evaluate the distance $r$ in the phase term in a Taylor series for small $x, y \ll z$. Without loss of generality we may assume $y=0$ and find

$$
\sqrt{z^{2}+x^{2}}=z+\frac{x^{2}}{2 z}-\frac{x^{4}}{8 z^{3}}+\mathcal{O}\left(x^{6}\right) .
$$


Ignoring the term of sixth order and accepting a maximum phase error of $\pi / 2$, the Fresnel approximation is valid as long as $\sqrt{x^{2}+z^{2}}-\left(z+x^{2} /(2 z)\right) \ll \lambda / 4$, i. e. for [Ree87]

$$
\frac{x^{4}}{2 z^{3}}<\lambda
$$

With the angle $\alpha=x / z$, the angular range in which the Fresnel approximation is valid is

$$
\alpha<\left(\frac{2 \lambda}{z}\right)^{\frac{1}{4}} .
$$

This angular range is very narrow for large distances. For instance, we find $\alpha_{\max } \approx 5.3 \mathrm{mrad}$ for $z=1 \mathrm{~m}$ and $\lambda=0.1 \mathrm{~nm}$. However, this value is larger than the typical divergence of the waveguide beam.

\subsection{Holography}

In principle, the optical transmission function of an object may be obtained from the wave penetrating the object. This wave may be reconstructed from the electric field incident on the detector. However, the detector only records intensity of the electric field; phase information is lost.

In holography, information of both amplitude and phase of the light wave originating from an object are stored by recording the intensity of an interference pattern of the object wave $S$ and a reference wave $R$. Gabor found out that the object wave may then be reconstructed by illuminating the hologram with the same reference wave Gab48.

Holography requires a high degree of both transverse coherence and monochromacity [PGW97]. In waveguide-based imaging, we may take transverse coherence as granted. This is evident for single-mode waveguides, since the electric field at the exit is determined by the fundamental mode. But the cross-sectional dimensions of multi-mode waveguides are usually also significantly smaller than the transverse coherence length of the beam incident on the waveguide. Accordingly, the waveguide is illuminated coherently and the exiting beam is coherent as well. Sufficient monochromacity is ensured by the use of double-bounce crystal monochromators with $\Delta \lambda / \lambda \approx 10^{-4}$.

The electric field in the hologram plane is given as a coherent superposition of the object wave $S(x, y)$ and the reference wave $R(x, y)$. The recorded intensity is then given by

$$
\begin{aligned}
I(x, y) & =|S(x, y)+R(x, y)|^{2} \\
& =R R^{*}+S S^{*}+R^{*} S+S^{*} R .
\end{aligned}
$$

In classical holography, the intensity pattern is recorded on a photographic film and the image is reconstructed by illuminating the negative with the reference wave. However, in our case it is much more convenient to reconstruct the wave numerically. Therefore, the 
optical transmission function of the hologram is assumed to be proportional to the recorded intensity:

$$
\tau_{H}(x, y) \propto I(x, y)
$$

We may then illuminate the hologram by a plane wave and find the electric field given by

$$
E_{R}(x, y) \propto R R^{*}+S S^{*}+R^{*} S+S^{*} R .
$$

Within this thesis, the reference wave $R$ is always a plane wave or a plane wave enveloped by the waveguide far-field diffraction pattern. Accordingly, the four terms on the righthand-side of Eq. (5.22) may be interpreted as follows:

- The first term represents a plane wave propagating in $z$ direction enveloped by the intensity pattern of the reference wave. In off-axis holography (Section 5.2.3), this is the zeroth-order beam.

- The second term represents a modulation of this plane wave by the intensity pattern of the object wave. Usually, this term is very small and can be ignored.

- The third term represents the object wave enveloped by the complex conjugate of the reference wave. This term contains the desired information about the imaged sample.

- The fourth term represents the complex conjugate of the object wave enveloped by $R$. This contribution results in the generation of a twin-image which significantly disturbs reconstruction from an in-line hologram (Section 5.2.1).

\subsubsection{In-line holography with a plane wave}

The most simple geometry of holographic recording is in-line holography (or Gabor holography) with a plane wave, as sketched in Fig. 5.4. In the recording process, the beam illuminating the sample also serves as a reference wave. This makes experiments comparably easy, since only one coherent beam must be provided. In the reconstruction, the object wave yields a direct image focused at the position where the imaged object was situated. However, this image is superposed by the reference wave modulated by $|S|^{2}$ and the conjugate wave. The conjugate wave in particular significantly disturbs the reconstructed image. It corresponds to a defocused image of a sample placed on the opposite side of the hologram, which is known as the "twin image". This image has the same intensity as the direct image and may significantly disturb the reconstruction. But since it is defocused, we may expect that objects with sharp edges may nevertheless be recognised in the reconstruction. 


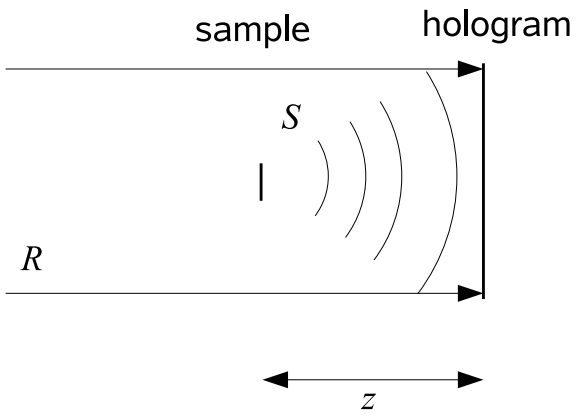

(a) Recording.

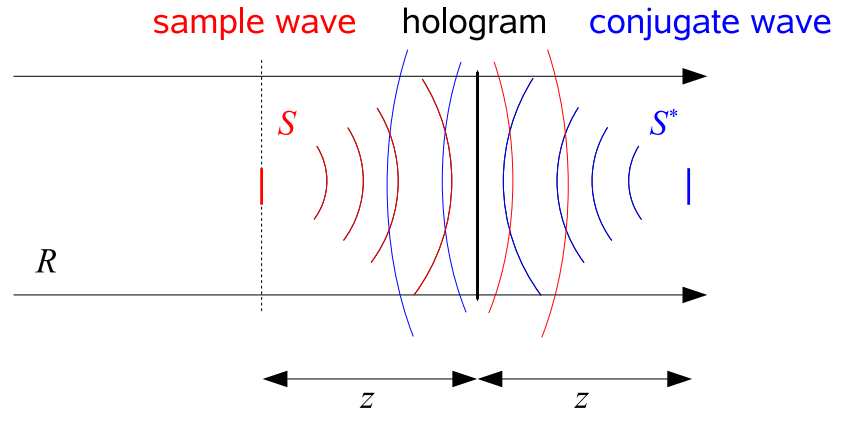

(b) Reconstruction.

Figure 5.4: Recording (a) and reconstruction (b) of a Gabor hologram. For reconstruction, the hologram is illuminated by the same reference wave $R$ as used for recording. The resulting field is (numerically) propagated to the plane where the object was situated (indicated by the dashed line). In this plane, the reconstructed object wave $S$ yields a focused image of the sample. However, this image is superposed by the slightly modulated reference wave and the conjugate wave $S^{*}$. The latter generates the twin image which significantly disturbs the reconstructed image. Note that the conjugate wave is focused on the opposite side.

\subsubsection{In-line holography with a point source}

Holography with a plane wave is not well-suited for high-resolution microscopy applications, since the recorded or reconstructed image is not magnified. The spatial resolution is thus limited to the detector pixel size. Therefore, we now consider holography with divergent beams provided by point-like sources. In the most simple case, namely Gabor microscopy, an in-line hologram is recording with a single point-like source.

Within the Fresnel approximation, it transpires that the hologram recorded with a point-source corresponds to a magnified hologram recorded with plane wave illumination Cow81] (see also Section A.2.1). Let $z_{1}$ denote the source-sample distance and $z_{2}$ the object-image distance (Fig. 5.5). Then, the recorded hologram is the same like a hologram recorded with a plane wave at an effective object-image distance of

$$
z_{\text {eff }}=\frac{z_{1} z_{2}}{z_{1}+z_{2}}
$$

magnified by a factor

$$
M=\frac{z_{1}+z_{2}}{z_{1}}
$$

Since the magnification factor is easily adapted by changing the corresponding distances, spatial resolution becomes independent of the detector pixel size. Note that a numerical reconstruction based on fast Fourier transformations is in practice only feasible after transformation to the case of plane-wave illumination. A direct propagation using fast Fourier transformations requires the same pixel size (or spatial resolution) on both the hologram and the object. The measured holograms typically cover $500 \times 500$ pixels on a detector with 


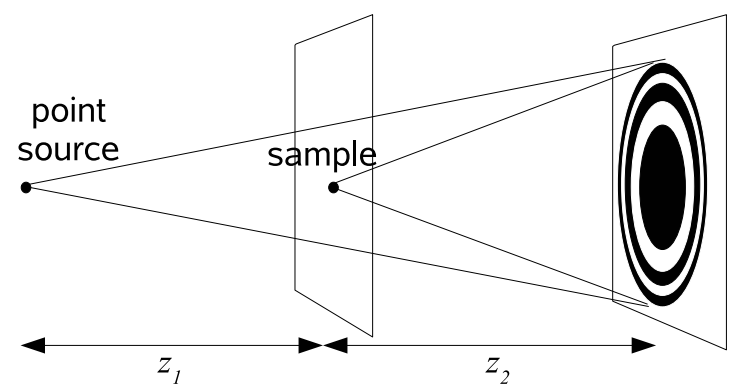

(a) Point-source holography.

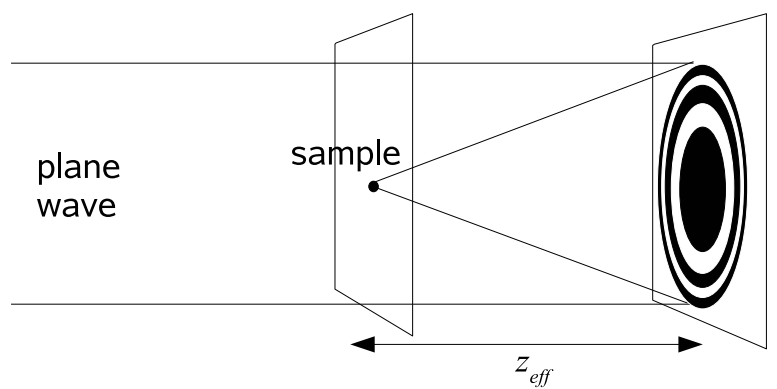

(b) Parallel-beam holography.

Figure 5.5: Within the Fresnel approximation, the hologram recorded with a point-source (a) transpires to be a magnified image of a hologram recorded with plane-wave illumination (b) at an effective focal distance $z_{\text {eff }}=z_{1} z_{2} /\left(z_{1}+z_{2}\right)$.

a pixel size of $20 \mu \mathrm{m}$. Demanding a spatial resolution of $100 \mathrm{~nm}$, a straightforward propagation using a spherical wave thus requires a calculation on $500 \times 20 \mu \mathrm{m} / 100 \mathrm{~nm}=10^{5}$ pixels in each direction, i. e. with a matrix of $10^{10}$ data points. This is practically infeasible on a standard PC.

\subsubsection{Off-axis holography}

In in-line holography, the reconstructed object wave is superposed by the twin image and reference wave contributions. While the reference wave may easily be eliminated in numerical reconstruction, the twin image forms an inseparable background. Since the conjugate wave has the same intensity as the object wave, the signal-to-noise ratio is equal to 1 and only sharp edges may be clearly distinguished from the defocused background.

In the 1960s, Leith and Upanieks presented a two-beam method that allowed for a spatial separation of the different waves in the reconstruction process [LU62, LU63]. The hologram is recorded with a second coherent reference beam, as shown in Fig. 5.6(a). If there is no sample in the beams, the recorded hologram is just a grating pattern resulting from interference of the two beams.

When illuminated with a plane wave, an off-axis hologram behaves like a diffraction grating, producing a pair of first-order diffracted beams (see Fig. 5.6(b)). One of these beams corresponds to the object wave and the other to the conjugate wave. In the plane where the object wave is focused, it is spatially separated from the reference beam as well as from the conjugate wave, i. e. the twin-image background is eliminated. This is illustrated in Fig. 5.7. Phase and intensity of the reconstructed first-order wave directly correspond to absorption and phase shift of the sample. Note that the zeroth-order beam corresponds to the result of an in-line hologram. Evidently, image quality is significantly improved in off-axis geometry.

In analogy to Gabor microscopy, off-axis holography can also be carried out using divergent beams from point-like sources [LUH65]. In a two-beam Gabor microscope (Fig. 5.8), 


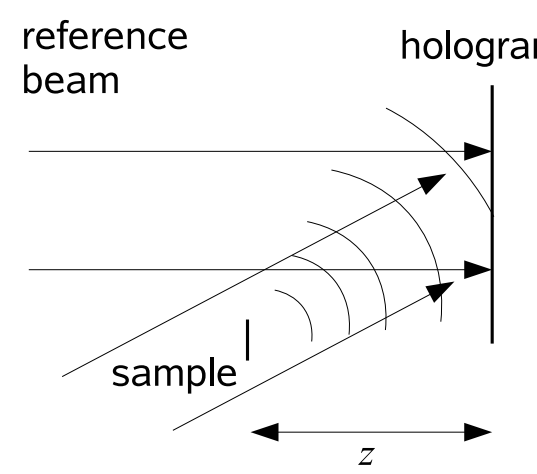

(a) Recording.

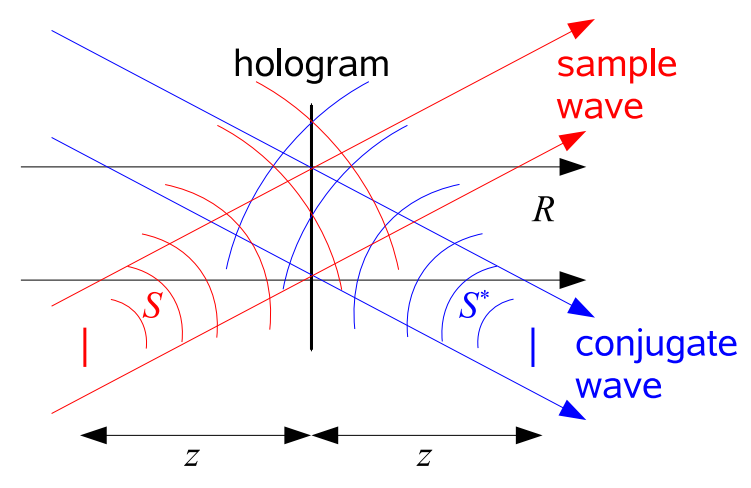

(b) Reconstruction.

Figure 5.6: Recording and reconstruction of an off-axis hologram. In the plane where the object wave is focused, it is spatially separated from the reference beam and the conjugate wave.

the beam originating from the sample interferes with the divergent beam of an additional point-like source displaced by a distance $d_{s}$. The recorded hologram then corresponds to a magnified version of a parallel-beam hologram recorded at an effective defocusing distance (see Section A.2.2

$$
z_{\text {eff }}=\frac{z_{1} z_{2}}{z_{1}+z_{2}}
$$

where $z_{1}$ and $z_{2}$ again denote the distances from the sample to the source and from the sample to the detector (Fig. 5.8). Magnification is again given by

$$
M=\frac{z_{1}+z_{2}}{z_{1}}
$$

and the reconstructed first-order beams include an angle

$$
\theta_{\text {eff }}=\frac{d_{S}}{z_{\text {eff }}}
$$

with the optical axis. Note that the field of view is limited to $d_{s}$ in the respective direction, since one has to ensure that the additional reference wave is not disturbed by the sample. Recording an off-axis hologram with the beams of two identical X-ray waveguides does not change the numerical aperture compared to in-line geometry, and so maximum spatial resolution is the same. However, off-axis geometry requires that the spatial resolution of the detector allows the resolving of the Young fringes (see Section 6.9.3).

Off-axis holography was applied to hard X-rays by Kohmura et al. using parallel beam illumination $\left[\mathrm{KS}^{+} 04\right]$. However, spatial resolution is thus limited by the pixel size and the field of view of the detector. Limited by the latter, a spatial resolution of $2.1 \times 1.3 \mu \mathrm{m}^{2}$ was achieved.

\subsubsection{Waveguide-based holography}

In this section, we analyse waveguide-based holography and highlight that the waveguide can indeed be considered a "point-like" source. Consider an X-ray waveguide with the 


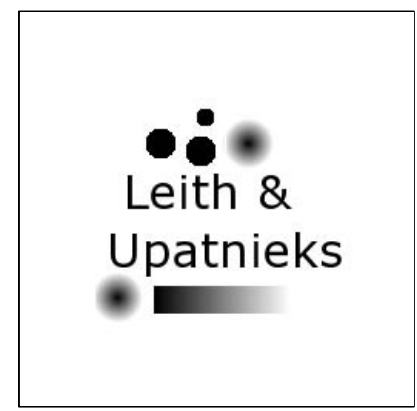

(a) Test object (dimensions $10 \times 10 \mu \mathrm{m}^{2}$, black corresponds to $\mathrm{Au}$ of $1 \mu \mathrm{m}$ thickness).

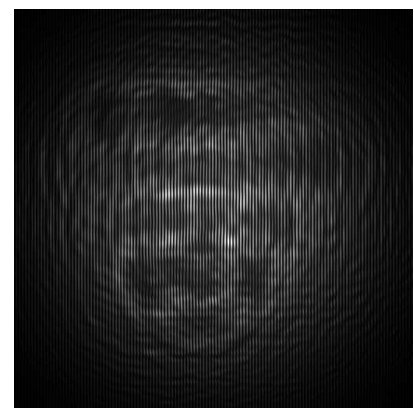

(b) Calculated off-axis hologram (Gaussian beams, $E=10.4 \mathrm{keV}, z=8 \mathrm{~mm}, \theta=$ $1.53 \mathrm{mrad})$.

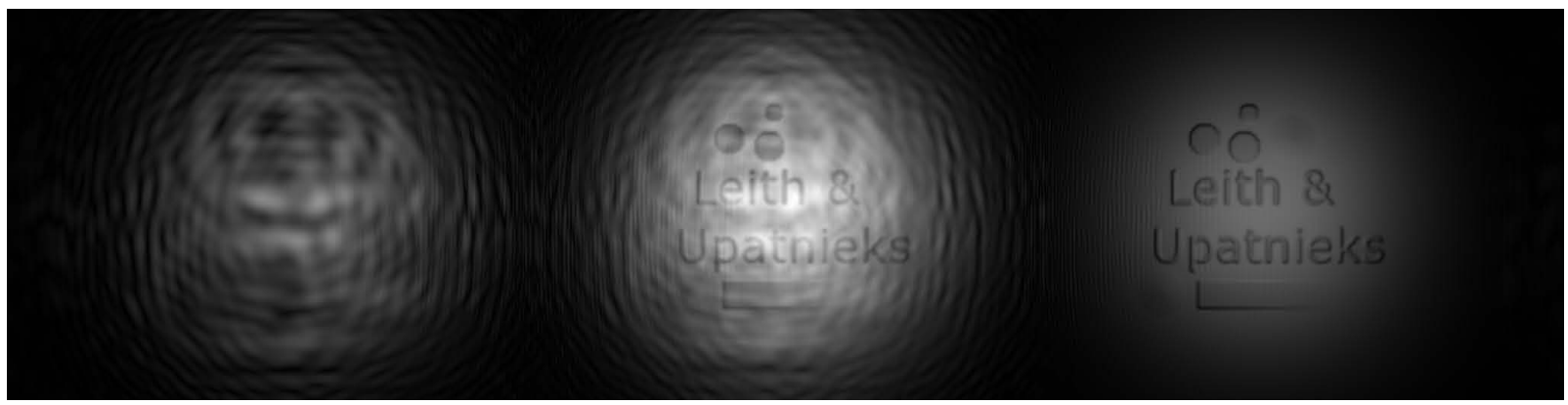

(c) Modulus of the reconstructed wave: The zeroth-order beam (middle) corresponds to a reconstructed Gabor in-line hologram. The first order beams show the reconstructed focused object wave (right) and a defocused image resulting from the conjugate wave (left).

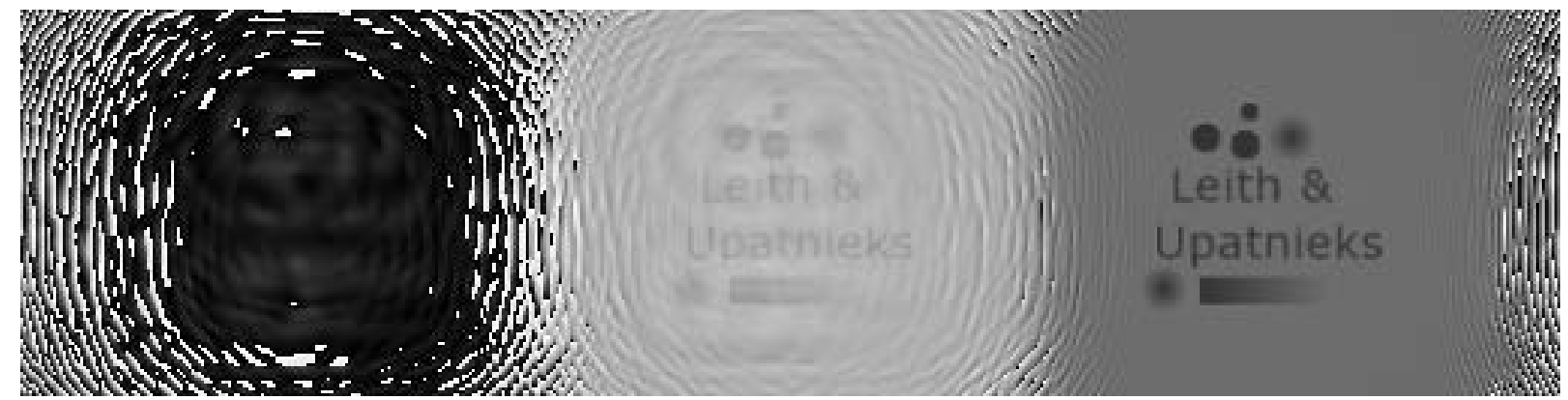

(d) Phase of the reconstructed wave: The reconstructed first order object wave (right) is evidently of much higher quality than the conventional Gabor hologram (zeroth-order beam, middle) and allows for a clearer identification of objects with fuzzy edges in particular. Due to the different propagation direction, the first-order beams are modulated in the horizontal direction by a term $\exp ( \pm i k \theta x)$. For better visibility, this term is eliminated by taking the averages over one period $2 \pi / k \theta$.

Figure 5.7: Calculated off-axis hologram and reconstruction of test structure. 


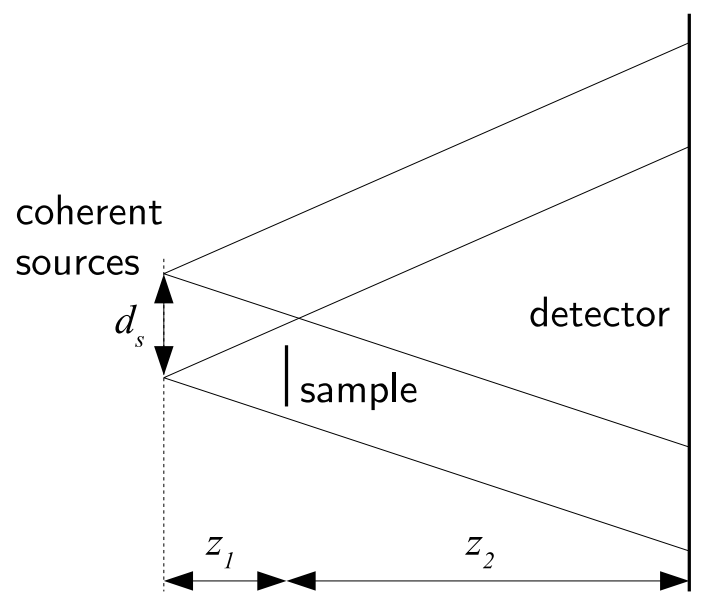

(a) Recording of a magnified off-axis hologram. The sample placed at distance $z_{1}$ from the respective source is illuminated by a divergent beam. A second source displaced by $d_{s}$ provides the reference beam. The hologram is recorded at distance $z_{2}$ behind the sample.

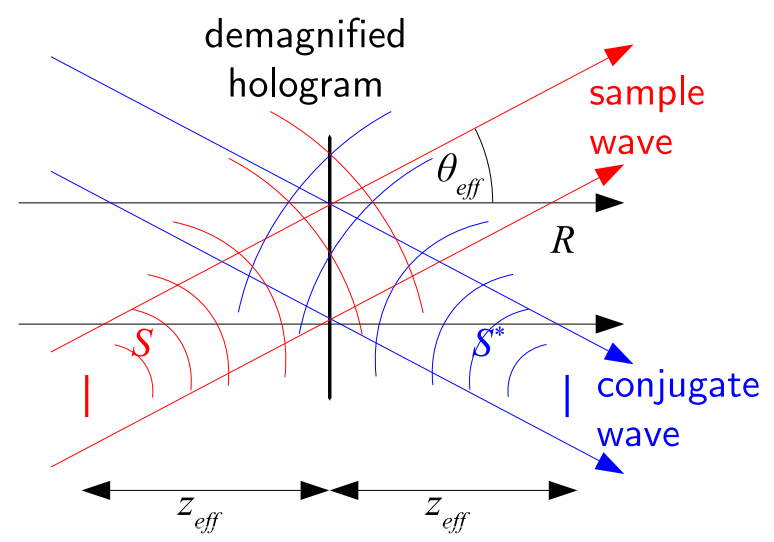

(b) For reconstruction, the hologram is demagnified by $M=\left(z_{1}+z_{2}\right) / z_{1}$. A parallel beam is used as the reference wave and the first-order beams propagate under an angle $\pm \theta_{\text {eff. }}$ The reconstructed waves are focused at $\pm z_{\text {eff }}=z_{1} z_{2} /\left(z_{1}+z_{2}\right)$, respectively.

Figure 5.8: The two-beam Gabor microscope enables the recording of a magnified off-axis hologram.

electric field at the end face given by $E_{w}\left(x_{w}, y_{w}\right)$. Within the Fresnel approximation, the electric field $E_{s}\left(x_{s}, y_{s}\right)$ incident on a sample at a distance $z_{1}$ is given by

$$
\begin{aligned}
E_{s}\left(x_{s}, y_{s}\right)= & \frac{\exp \left(i k z_{1}\right)}{i \lambda z_{1}} \int E_{w}\left(x_{w}, y_{w}\right) \exp \left(i k \frac{\left(x_{s}-x_{w}\right)^{2}+\left(y_{s}-y_{w}\right)^{2}}{2 z_{1}}\right) \mathrm{d} x_{w} \mathrm{~d} y_{w} \\
= & \frac{\exp \left(i k z_{1}\right)}{i \lambda z_{1}} \int E_{w}\left(x_{w}, y_{w}\right) \\
& \quad \times \exp \left(i k \frac{x_{s}{ }^{2}+y_{s}{ }^{2}+2\left(x_{s} x_{w}+y_{s} y_{w}\right)+x_{w}{ }^{2}+y_{w}{ }^{2}}{2 z_{1}}\right) \mathrm{d} x_{w} \mathrm{~d} y_{w} .
\end{aligned}
$$

Since the field at the end face of the waveguide is practically zero far away from the guiding core, we may only consider $E_{w}$ where $x_{w}$ and $y_{w}$ are not much greater than the guiding core dimensions. There, we typically find

$$
k \frac{x_{w}^{2}}{2 z_{1}} \ll 1, \quad k \frac{y_{w}^{2}}{2 z_{1}} \ll 1 .
$$

(Consider for instance $x_{w}=50 \mathrm{~nm}, z_{1}=1 \mathrm{~mm}, \lambda=0.1 \mathrm{~nm}$. In this case, the term on the left hand side is $\approx 0.08$, further decreasing for larger distances). Ignoring these terms, we find the approximation

$$
E_{s}\left(x_{s}, y_{s}\right) \approx \underbrace{\frac{\exp \left(i k z_{1}\right)}{i \lambda z_{1}} \exp \left(i k \frac{x_{s}^{2}+y_{s}^{2}}{2 z_{1}}\right)}_{\text {field of a point source }} \underbrace{\int E_{w}\left(x_{w}, y_{w}\right) \exp \left(i k \frac{\left(x_{s} x_{w}+y_{s} y_{w}\right)}{z_{1}}\right) d x_{w} d y_{w}}_{\text {waveguide far-field diffraction pattern }} .
$$


The electric field incident on the sample is thus the electric field of a point source multiplied by the complex far-field diffraction pattern of the waveguide. If we assume that the field at the end face of the waveguide is symmetric, i. e. $E_{w}\left(x_{w}, y_{w}\right)=E_{w}\left(-x_{w},-y_{w}\right)$, the imaginary part of the function integrated in the second term is antisymmetric:

$$
E_{w}\left(x_{w}, y_{w}\right) \sin \left(i k_{0} \frac{\left(x_{s} x_{w}+y_{s} y_{w}\right)}{z_{w s}}\right)=-E_{w}\left(-x_{w},-y_{w}\right) \sin \left(i k_{0} \frac{\left(-x_{s} x_{w}-y_{s} y_{w}\right)}{z_{w s}}\right) .
$$

Accordingly, the imaginary part of the integral vanishes and we can write

$$
E_{s}\left(x_{s}, y_{s}\right)=E_{s}^{\text {point source }}\left(x_{s}, y_{s}\right) \times \sqrt{I^{\mathrm{WG} \text { far-field }}\left(x_{s}, y_{s}\right)}
$$

where $E_{s}^{\text {point source }}\left(x_{s}, y_{s}\right)$ denotes the field of a point source and $I^{\mathrm{WG} \text { far-field }}\left(x_{s}, y_{s}\right)$ denotes intensity in the waveguide far-field. The reference wave in waveguide-based holography may thus be described as a spherical wave enveloped by the waveguide far-field diffraction pattern. The far-field diffraction pattern limits the numerical aperture NA in holographic imaging. The achievable spatial resolution is given by

$$
s \approx \frac{\lambda}{2 \mathrm{NA}}
$$

where NA is determined by the divergence of the waveguide beam. The latter is limited by the critical angle of total reflection $\theta_{c}$ which is the numerical aperture of a single-mode waveguide. The resolution limit is thus given by

$$
s \approx \frac{\lambda}{2 \theta_{c}}=W
$$

which is the critical width of planar waveguide as defined in Section 2.7. With increasing guiding core dimensions, the numerical aperture decreases. Considering only the fundamental mode, the numerical aperture approximately decreases inversely proportional to the core diameter. Spatial resolution for imaging experiments is then approximately given by the cross-sectional dimensions of the guiding core.

\subsection{Practical considerations}

In this section we consider the practical aspects of waveguide-based imaging. Different imaging regimes depending on the effective defocusing distance are illustrated.

\subsubsection{Imaging regimes in waveguide-based imaging}

Depending on the effective defocusing distance, the detected image may give some kind of "direct" contrast (i. e. the detected intensity distribution corresponds directly to absorption or the Laplacian of the phase shift) or a more holographic image showing only little resemblance to the investigated object. The accessible spatial frequencies are furthermore limited by 
- Resolution of the waveguide: The accessible spatial frequencies are limited by the spatial resolution $s$ of the waveguide which, at least for single-mode waveguides, approximately corresponds to the diameter $d$ of the guiding core. The maximum resolvable frequency is then given by

$$
\nu_{\max }=\frac{1}{s} \approx \frac{1}{d}
$$

- Field of view: On the other end, the spatial frequencies are limited by the accessible field of view, i. e. the low divergence $\alpha$ of the waveguide. In a distance $z_{1}$ the field of view is $\approx \alpha z_{1}$. With $z_{\text {eff }} \approx z_{1}$ we find

$$
\nu_{\min } \approx \frac{1}{\alpha z_{\mathrm{eff}}}
$$

Note that the field of view may be enlarged by recording multiple holograms at different positions and stitching the results.

- Resolution of the detector: Due to the finite detector pixel size, the spatial resolution may also be limited by the detector pixel size. Typically, a CCD detector is placed at a fixed distance and the area covered by the waveguide far-field diffraction pattern does not depend on the effective defocusing distance. Assuming that the detector resolves $N$ pixels in each direction in the waveguide far-field, the corresponding resolution limit is given by the field of view divided by $N$. Correspondingly, the highest resolvable spatial frequency is

$$
\nu_{\mathrm{det}, \max }=\frac{N}{\alpha z_{\mathrm{eff}}} .
$$

We now consider the different imaging regimes in waveguide-based imaging. The waveguide is considered as a point-like source. As shown in Section 5.1.2, the different imaging regimes depend on the reduced squared frequency $\chi=\pi \lambda z \nu^{2}$. Depending on the effective defocusing distance $z$, we find some kind of "direct contrast" for frequencies below $\chi=\pi / 2$.

Fig. 5.9 illustrates the different regimes for a waveguide providing a divergence of $1 \mathrm{mrad}$ at an X-ray wavelength of $0.1 \mathrm{~nm}$. The corresponding spatial resolution is then $s \approx$ $\lambda / 2 \mathrm{NA}=50 \mathrm{~nm}$. Evidently, highest spatial resolution requires holographic reconstruction. 


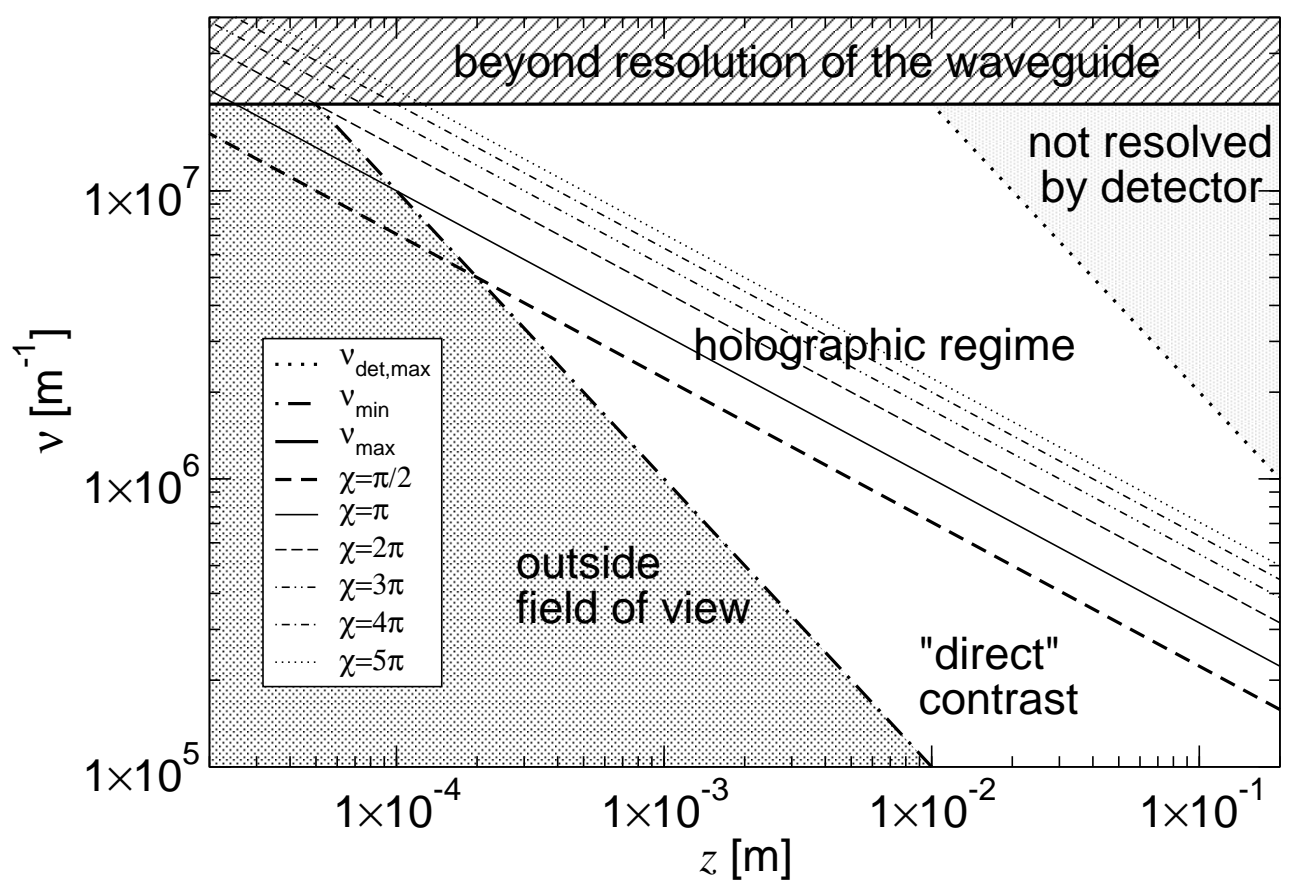

Figure 5.9: The different imaging regimes in waveguide-based imaging assuming a waveguide with a numerical aperture of $1 \mathrm{mrad}$, an $X$-ray wavelength of $0.1 \mathrm{~nm}$, and a detector resolving 200 pixels in the waveguide far-field diffraction pattern. $\chi$ indicates the reduced squared frequency $\chi=\pi \lambda z \nu^{2}$. 


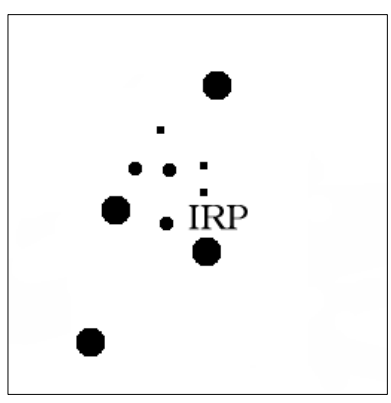

(a)

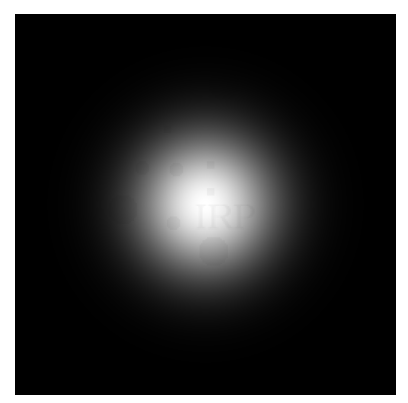

(b)

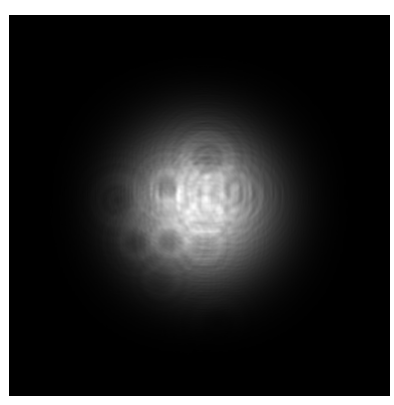

(c)

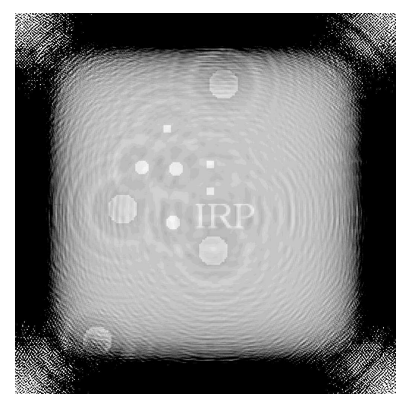

(d)

Figure 5.10: Calculated in-line holography experiment with a test structure prepared in an Au layer of $100 \mathrm{~nm}$ thickness (a): calculated field intensity directly downstream of the sample (b), calculated in-line hologram (c), and phase of the reconstruction (d). The dimensions of the images are $20 \times 20 \mu^{2}$.

\subsubsection{Noise}

Although modern synchrotron X-ray sources provide highly intensive and brilliant beams, brilliance is still low compared to lasers emitting visible light. For illustration, consider the following example: A test structure prepared in a thin gold layer with a thickness of $100 \mathrm{~nm}$ (Fig. 5.10(a) with dimensions of $20 \times 20 \mu \mathrm{m}^{2}$ is illuminated by a Gaussian shaped beam with a full width at half maximum of $2 \mathrm{mrad}$. Let the sample be situated $5 \mathrm{~mm}$ downstream of the waveguide and assume a detector with $1024 \times 1024$ pixels at a distance of $1 \mathrm{~m}$. Fig. 5.10 shows the resulting field intensity behind the sample (Fig. $5.10(\mathrm{~b})$, a calculated in-line hologram (Fig. $5.10(\mathrm{c})$, and the phase image of the respective reconstruction (Fig. $5.10(\mathrm{~d})$ ) for a photon energy of $8 \mathrm{keV}$. At this photon energy, transmission of the gold layer is $\approx 96.1 \%$ and phase shift is $\approx 0.19 \mathrm{rad}$. The reconstruction was carried out with a plane reference wave and the twin-image appears as background in the reconstruction. Although no noise was included in the calculations, finite numerical accuracy of the calculations leads to unusable results in the outermost regions of the reconstruction, where intensity is very low.

Fig. 5.11 shows calculated holograms and reconstructions assuming a limited number of photons and Poisson statistics. The calculations are carried out for a flux of 500, 50 and 10 photons per pixel in the centre of the beam. For highest flux, practically all structures are clearly resolved. Only the circle in the lower left is too far away from the beam and hidden in noise. With further decreasing flux, the reconstruction becomes noisier and the region where the reconstruction gives usable results shrinks. However, even with a flux of only 10 photons per pixel, the structures in the centre of the beam can be clearly identified.

In conclusion, finite flux causes noise in the reconstruction, but even with only 10 photons per pixel the reconstruction may yield usable results. In the given example, a flux of 50 photons per pixel in the centre of the beam seems sufficient to image all structures within the full width at half maximum of the beam with high quality. 

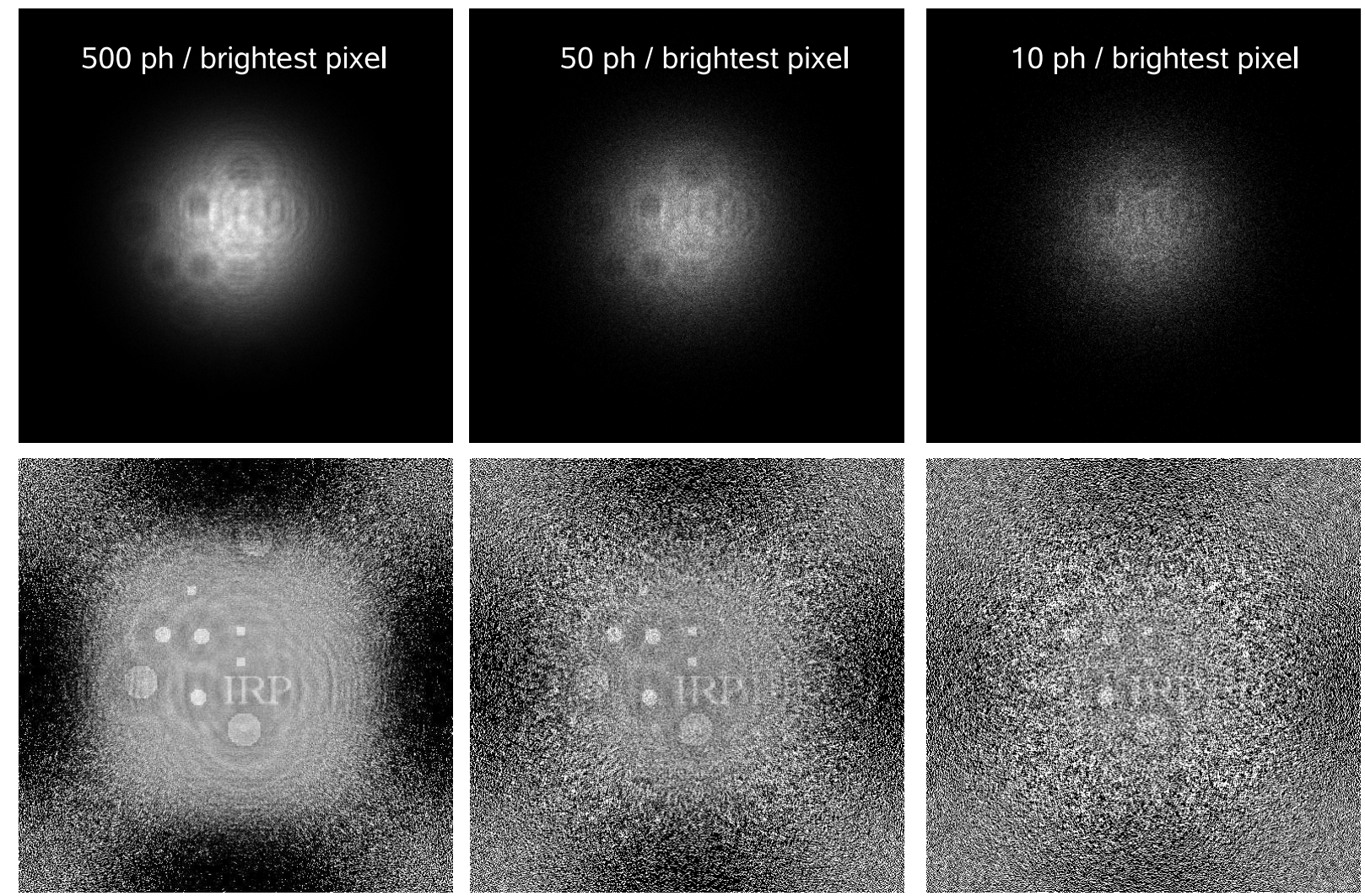

Figure 5.11: Calculated holograms of the test structure shown in Fig. 5.10. The holograms in the upper row are calculated with Poissonian noise assuming 500, 50, and 10 photons per pixels, respectively, in the centre of the beam. The lower row shows the corresponding phase of the reconstructed waves. 


\subsection{Conclusions}

In conclusion, an X-ray waveguide can be considered as a point-like source and the recorded image is described by scalar diffraction theory (Section 5.1). However, in comparison to a real point-source, the waveguide has a limited numerical aperture since the recorded image is enveloped by the waveguide far-field diffraction pattern (Section 5.2.4). The limited numerical aperture thus limits spatial resolution to approximately the waveguide cross-section dimensions.

Depending on the feature size of the imaged sample and the distance to the waveguide, a recorded image may already provide some kind of "direct" contrast or has to be considered as an in-line hologram with little direct resemblance to the investigated object (Section 5.3.1). In the latter case, in particular when the feature size is close to the resolution limit, a holographic reconstruction is necessary. Due to the loss of phase information in in-line holography, the reconstructed image is superposed by a conjugate wave, which significantly spoils the image. Much better unambiguous images may be obtained when the hologram is recorded with an additional reference beam (Section 5.2.3) that may be experimentally realised using two coherently illuminated X-ray waveguides.

In real experiments, image quality is also affected by the limited number of photons exiting the waveguide, inevitably introducing Poissonian noise. Although this also introduces noise in the reconstructed wave, the calculations shown in Section 5.3 .2 indicate that satisfactory results may be achieved with $10^{2}-10^{3}$ photons per pixel. 


\section{Experiments I: Characterisation of X-ray waveguides}

This chapter describes the fabrication and characterisation of X-ray waveguides. We start with some general considerations concerning the design of waveguides for practical applications (Section 6.1). Section 6.2 then describes the fabrication of X-ray waveguides. In particular, the preparation of two-dimensionally confining waveguides by means of electronbeam lithography is presented. Section 6.3 presents a brief survey of the different coupling schemes used for X-ray waveguides and highlights the advantages of direct coupling applied in the present experiments. Direct coupling into single-mode planar waveguides is then demonstrated in Section 6.4. In Section 6.5 we study the propagation of white X-rays in a planar waveguide. The white-beam experiments demonstrate coherent propagation and allow an experimental verification of the propagation constants for slab waveguides.

In Section 6.6, direct coupling into two-dimensionally confining waveguides is demonstrated. To obtain sufficient flux, the primary beam is pre-focused by a system of crossed mirrors in the Kirkpatrick-Baez geometry. Alternatively, arrays of two-dimensionally confining waveguides are characterised in unfocused parallel beams, allowing a characterisation without the need for an extremely high density of flux (Section 6.7). Section 6.8 demonstrates how the density of flux at the exit of two-dimensionally confining waveguides is further increased in tapered waveguides.

Finally, we demonstrate true guiding of X-rays in the sense of changing the direction of propagation. We study curved waveguides that allow the spatial separation of the end-faces of two coherently illuminated waveguides. Under appropriate experimental conditions, these waveguides provide two spatially separated coherent beams enabling new kinds of experiments, like off-axis holography (Section 6.9).

\subsection{Waveguide design}

The design parameters for X-ray waveguides include the choice of the materials, the length of the waveguide, and the cross-sectional dimensions of the guiding core. These parameters have to be chosen with consideration of the flux demanded from the exiting beam, the density of flux provided by the incident beam, and technical aspects of fabrication.

The choice of materials in particular determines the smallest cross-sectional dimensions of the guiding core and thus spatial resolution in waveguide-based imaging or scanningprobe microscopy. The choice of the cross-sectional dimensions $d$ is also guided by flux considerations. In particular, flux incident on the front face of a two-dimensionally confin- 
ing waveguide scales with $d^{2}$. For small $d$, flux exiting the device decreases even faster due to the decreasing transmission at small cross-sectional dimensions (see Section 4.1).

Taking into account the technical aspects of fabrication, a silicon cladding and a polymer guiding core were chosen. The corresponding lower limit of the guiding core dimensions is $W \approx 30 \mathrm{~nm}$. The typical cross-section dimensions for two-dimensionally confining waveguides are about $50 \mathrm{~nm}$, chosen as a compromise between flux and spatial resolution. Furthermore, these waveguides support only a few guided modes and the far-field is dominated by the Gaussian-like contribution of the fundamental mode.

The length of the waveguides has to be long enough to provide sufficient absorption in the cladding, but not so long as to minimise absorption in the guiding core. Evidently, the optimal length thus depends crucially on the photon energy. The length is typically adapted to a transmission of $10^{-8}-10^{-6}$ in the cladding and $\approx 0.1$ in the waveguide, corresponding to $1-5 \mathrm{~mm}$ at a photon energy between 8 and $13 \mathrm{keV}$.

\subsection{Fabrication of X-ray waveguides}

The X-ray waveguides presented in this thesis consist of a polymer guiding core in a silicon cladding. A thin polymer layer is prepared on a standard substrate by spin-coating technique. In the case of two-dimensionally confining waveguides, the polymer guiding core is structured by electron beam lithography [Mad02] (Fig. 6.1). The electron beam lithography system Leica LION LV 1 (Lithography System for Integrated Optics and Nanometric Structures, Low Voltage $1 \mathrm{keV}$ ) [Lei97], which allows the exposure of continuous lines with a length of up to several centimetres, is used. The system may also be used as a scanning electron microscope [Rei98].

Two different polymer resists are used: Calixarene (Allresist XAR-N7600) and poly (methyl methacrylate) (PMMA, Elvacite 2041). Calixarene is a negative resist, i.e. the exposed regions remain after development (as shown in Fig. 6.1(a)), while PMMA is a positive resist and the exposed regions are removed during development. Calixarene waveguides are developed for $45 \mathrm{~s}$ in the associated developer XAR-600-59 and the process is stopped by rinsing for $15 \mathrm{~s}$ in the stopper AR-600-60. PMMA waveguides are developed for $45 \mathrm{~s}$ in a 4:1 mixture of ethylene glycol monoethyl ether and ethylene glycol monobutyl ether. Here, the process is stopped in 2-propanol. Furthermore, planar waveguides were prepared with a polyimide (HD Microsystems PI-2545) guiding layer.

The height of two-dimensionally confining waveguides is determined by the thickness of the resist layer. This thickness is precisely measured by means of X-ray reflectivity using a Bruker D8 Advance diffractometer and fitting the results with the IMD software Win98. However, height of the structures may be changed by the development process.

The minimum width of the waveguides is determined by the resolution of the resist. Sub-10 nm resolution has been demonstrated for both calixarene [FOOM96, $\mathrm{SFO}^{+} 99$, IFO ${ }^{+}$3] and PMMA [CA93. Typically, the waveguides are prepared by exposing one 


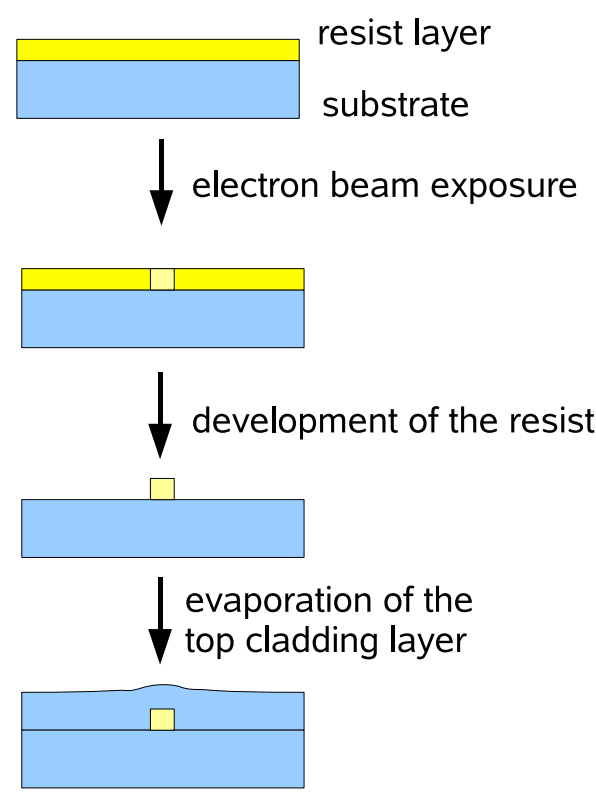

(a)

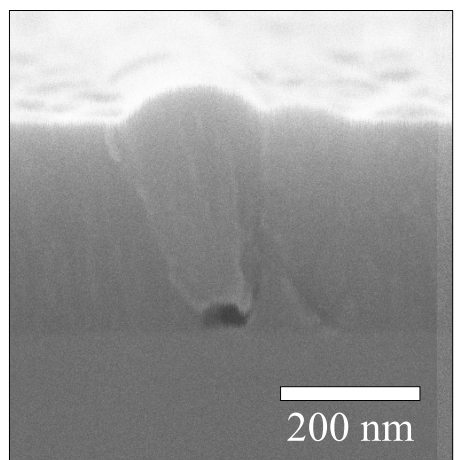

(b)

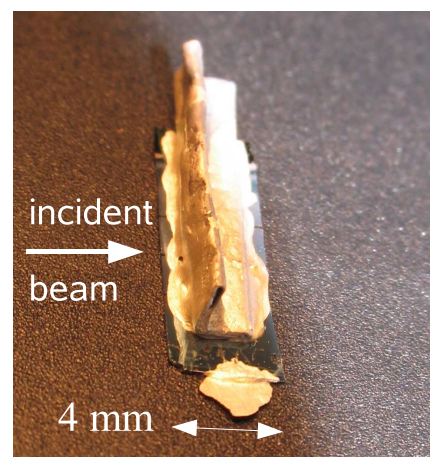

(c)

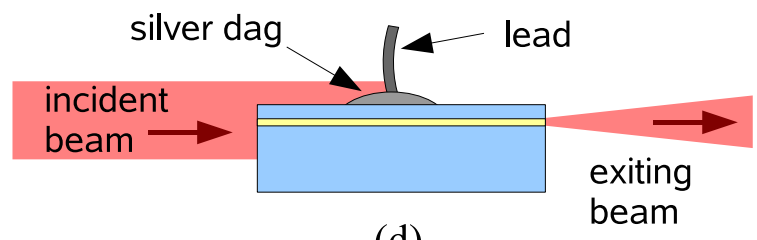

(d)

Figure 6.1: Sketch of the fabrication of two-dimensionally confining waveguides by electron beam lithography (a), scanning electron microscopy image of a two-dimensionally confining waveguide (b), photograph of a waveguide (c), and blocking of the primary beam and scattered radiation by a small piece of lead tape fixed with silver dag $(d)$.

or multiple parallel lines spaced by $15-20 \mathrm{~nm}$ with a line dose of $1-2 \mathrm{nC} / \mathrm{cm}$ (calixarene) or 0.1-0.2 nC/cm (PMMA). Alternatively, line width of individual lines may be increased by well-defined defocusing of the electron beam [DH99]. The lower dose required for PMMA makes it faster to write large gratings in PMMA, while calixarene as a negative resist is favoured for writing individual two-dimensionally confining waveguides typically spaced by $20 \mu \mathrm{m}$.

The top silicon layer is deposited by electron beam evaporation (Leybold Univex 450). The thickness of the top cladding layer has to be large compared to the penetration depth of the evanescent wave propagating in the cladding and is typically 200-300 $\mathrm{nm}$. The waveguides are then cleaved to an appropriate length. The end faces of the waveguides used for the imaging experiments presented in Chapter 7 were "polished" by focused ion beams at the Institut für Materialphysik (University of Göttingen). Since the cross-sectional dimensions of the incident beam are typically much larger than the thickness of the top cladding layer, a small piece of lead tape is fixed atop the waveguide with silver dag (Conrad Silberleitlack) to block out radiation passing above (Fig. 6.1(d)).

There have also been other attempts to fabricate two-dimensionally confining waveguides, like coated spider-silk, small holes drilled in metal foils by means of focused ion beams, or wafer-bonding of Si substrates in which U- or V-grooves are etched [Jar05, See04, $\mathrm{PJB}^{+}$05. However, the best results concerning both small cross-sectional dimensions and reliability have so far been obtained using the presented polymer waveguides. 


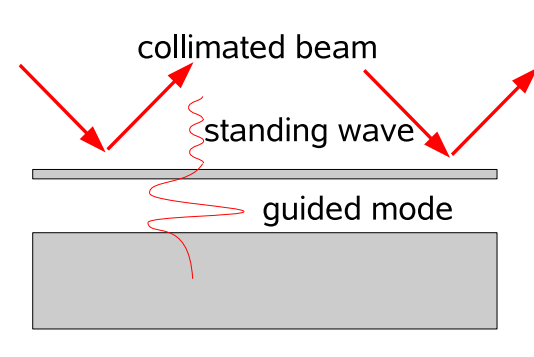

(a)

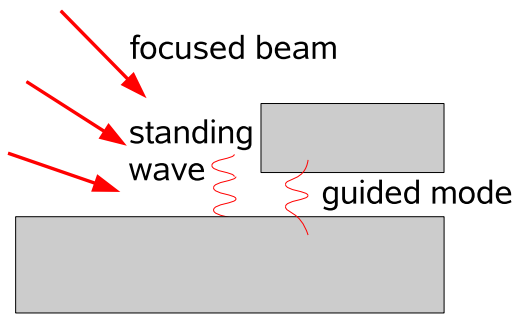

(b)

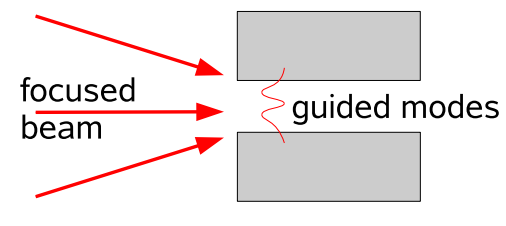

(c)

Figure 6.2: Coupling schemes for X-ray waveguides: In the resonant beam coupler scheme a highly collimated incident beam resonantly excites a guided mode through a thin upper cladding layer (a). A focused beam may be coupled into the waveguide via a standing wave above the substrate (b) or directly into the front face of the waveguide (c).

\subsection{Coupling of X-rays into waveguides}

Typical cross-sectional dimensions of X-ray beams provided by synchrotron beamlines or tube sources are in the millimetre range and thus far above the typical cross-sectional dimensions of an X-ray waveguide. Accordingly, it is quite difficult to couple sufficient intensity from the primary beam into a comparatively small guiding core. The first experiments carried out with X-ray waveguides applied the "resonant beam coupler" scheme, where the guided modes of a waveguide are excited via an evanescent wave through a thin upper cladding layer [SS73, FSD $^{+}$93]. At certain incidence angles, resonance effects then lead to an increase of flux density in the guiding core of up to two orders of magnitude compared to the primary beam $\left[\mathrm{JCF}^{+} 01\right]$ (Fig. 6.2(a) . However, the grazing angle of the primary beam has to match these angles almost exactly and efficient coupling requires a well-collimated beam. Accordingly, this coupling scheme is not very efficient using a pre-focused, i. e. convergent, beam.

Since state-of-the-art hard-X-ray focusing optics like for instance Kirkpatrick-Baez mirrors [KB48, $\left.\mathrm{HCL}^{+} 03\right]$ may today increase flux density by more than five orders of magnitude, it is more favourable to use these optics as pre-focusing devices. Coupling of a pre-focused beam into planar waveguides has therefore already been demonstrated using linear Fresnel-zone-plate $\left[\mathrm{BDD}^{+} 02\right]$ or compound refractive lenses [SSS${ }^{+} 03$ ] via a standing wave above the substrate (Fig. 6.2(b)).

However, the first experiments with two-dimensionally confining waveguides were again carried out using the resonant beam coupler scheme $\left[\mathrm{PDB}^{+} 02\right]$. Besides the fact that this method may be difficult to apply to non-rectangularly shaped waveguides, intensity was too low for reasonable applications and the waveguide beam was superposed by disturbing contribution of the transmitted and specularly reflected primary beams.

As a practical solution for both one-dimensionally and two-dimensionally confining waveguides, a pre-focused beam may be coupled directly into the front side of the waveguide 
(Fig. 6.2(c) ). Since absorption in the cladding is typically much higher than in the guiding layer, the ratio of intensity transmitted through the guiding layer to intensity transmitted beside increases with increasing length of the waveguide. However, since also the guided beam is subject to significant absorption, a high ratio significantly decreases flux. To get both high flux and low background, the pre-focusing optics must provide a focus not too much larger than the dimensions of the waveguide.

\subsection{Direct coupling into a single-mode planar waveguide}

This experiment demonstrates direct coupling of a focused synchrotron beam into the front face of a planar single-mode waveguide. Locating the waveguide in the focus of a pre-focusing device allows the combination of the high density of flux provided by the focusing device with an even smaller beam size and well-defined intensity distribution of a single-mode waveguide. Monochromatic flux exceeding $10^{8}$ photons per second is thus obtained from a planar waveguide with a guiding layer thickness of $32 \mathrm{~nm}$.

\subsubsection{Experiment}

The experiment was carried out at the undulator beamline ID $22\left[\mathrm{STMC}^{+} 05\right]$ of the European Synchrotron Radiation Facility (ESRF) in Grenoble, France. The experiment setup is sketched in Fig. 6.3. The X-ray beam with a photon energy of $12.5 \mathrm{keV}$ and a cross-section of $1 \mathrm{~mm}$ was focused using a crossed pair of Kirkpatrick-Baez (KB) mirrors [DRH ${ }^{+} 02$ ] with focal lengths of $0.2 \mathrm{~m}$ (horizontal focusing) and $0.4 \mathrm{~m}$ (vertical focusing), resulting in a beam convergence of $\approx 0.14^{\circ}$ in vertical direction. To reduce the background, a $20 \mu \mathrm{m}$ Pt pinhole was placed in front of the waveguide. The waveguide consists of a polyimide $\left(\mathrm{C}_{22} \mathrm{H}_{10} \mathrm{~N}_{2} \mathrm{O}_{5}\right)$ guiding layer with $32.3 \mathrm{~nm}$ thickness in Si. The waveguide was cleaved to a length of $\ell \approx 4 \mathrm{~mm}$, resulting in a primary beam transmission of $\approx 2 \times 10^{-7}$ in the $\mathrm{Si}$ cladding, while finite-difference calculations predict a transmission of $\approx 0.09$ for the guided beam (Fig. 6.3).

The entrance of the waveguide was placed in the focus of the KB mirrors where flux behind the pinhole was $1.7 \times 10^{11} \mathrm{ph} / \mathrm{s}$. The focal spot size was determined by a knife-edge scan to be $2.5 \mu \mathrm{m}$ in vertical and $3.8 \mu \mathrm{m}$ in horizontal direction. Therefore, fluorescence of a gold stripe evaporated on a silicon substrate was measured with an energy dispersive $\mathrm{Si}$-Li detector. Accordingly, the focal spot was approximately 80 times larger in vertical direction than the guiding layer thickness, resulting in a flux of $2.2 \times 10^{9} \mathrm{ph} / \mathrm{s}$ at the entrance. The far-field diffraction pattern was measured using a fast scintillation detector (Cyberstar, Oxford Instruments) placed $43 \mathrm{~cm}$ behind the waveguide. 


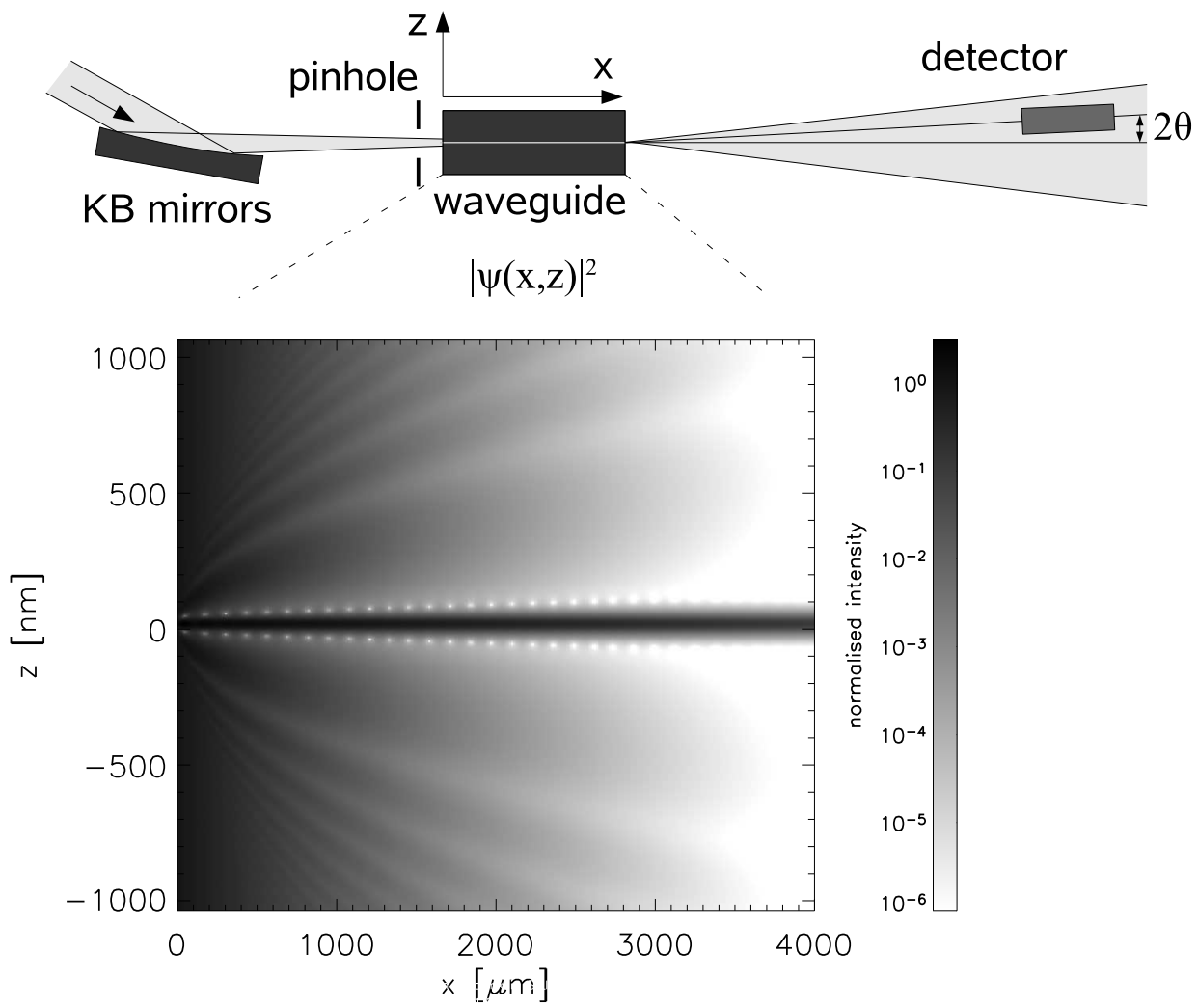

Figure 6.3: Experiment setup (top): The X-ray beam is focused by a Kirkpatrick-Baez (KB) mirror system and directly coupled into the front face of the waveguide. A logarithmic grey-scale image of intensity inside the single-mode waveguide (bottom) demonstrates strong attenuation in the cladding, i.e. the electric field at the exit of the waveguide is dominated by the guided fundamental mode. Intensity was obtained from a finite-difference calculation; distances and angles are not to scale. 


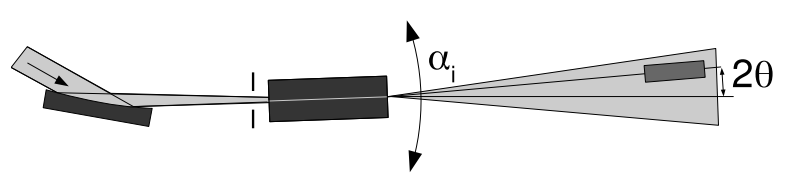

(a)

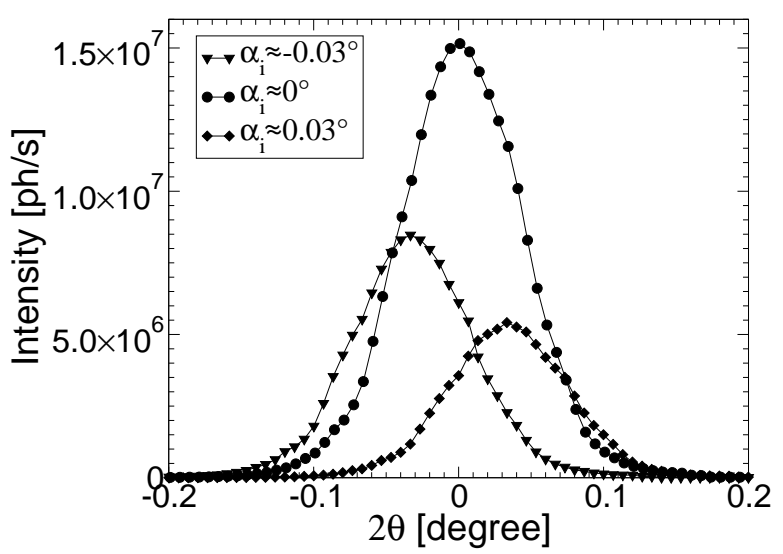

(b)

Figure 6.4: Variation of the angle of incidence $\alpha_{i}$ (a) shifts the angular position of the far-field diffraction pattern with respect to the primary beam by the same amount (b). The intensity decreases significantly when the angle of incidence $\alpha_{i}$ is changed by $0.03^{\circ}$ above or below the optimal position of normal incidence.

\subsubsection{Results}

Total flux exiting the waveguide was $1.5 \times 10^{8} \mathrm{ph} / \mathrm{s}$ in a cross-section of $3.8 \mu \mathrm{m}$ (horizontal, defined by the KB mirrors) $\times 32 \mathrm{~nm}$ (vertical, defined by the guiding layer), corresponding to a transmission of $7 \%$ and a gain in flux density of $1.5 \times 10^{3}$ compared to the beam in front of the KB mirrors. This is an order of magnitude higher than the typical gain of high performance planar waveguides in the resonant beam coupler scheme [ $\left.\mathrm{JCF}^{+} 01\right]$.

Fig. 6.3 shows a finite-difference calculation of the electric field inside the waveguide. The electron density of the polyimide layer is assumed to be determined by a mass density of $\rho=1.42 \mathrm{~g} / \mathrm{cm}^{3}$ [HGD93]. The logarithmic grey-scale image illustrates rapid decay of intensity in the cladding and much weaker absorption in the guiding layer. The transmission is $\approx 9.4 \%$ for X-rays impinging at normal incidence. Taking into account a convergence angle of $0.14^{\circ}$ for the incident beam, this value reduces to $\approx 7 \%$, in good agreement with the measurement. As analytically shown in Section 4.2, the measured transmission of the single-mode waveguide is thus indeed significantly lower than that of bulk polyimide of the same length, which is $\approx 46 \%$.

In Fig. 6.4, the measured far-field diffraction patterns for different angles of incidence $\alpha_{i}$ are shown as a function of the exit angle $2 \theta$ with respect to the incident beam. Since the waveguide supports only one guided mode, a variation of the angle of incidence does not change the shape of the far-field pattern nor its angular position with respect to the waveguide axis. However, total intensity decreases with increasing $\left|\alpha_{i}\right|$ and the angular position changes with respect to the primary beam by the same amount as $\alpha_{i}$. 


\subsubsection{Conclusions}

A focused monochromatic X-ray beam was coupled directly into the front side of a planar single-mode waveguide. Flux behind the waveguide exceeded $10^{8} \mathrm{ph} / \mathrm{s}$, while there were no significant contributions of radiation transmitted through the cladding. Accordingly, the combination of a pre-focusing device and an X-ray waveguide indeed provides very small and well-defined coherent beams with high flux and a high signal-to-noise ratio. The method is extended to two-dimensionally confining waveguides in Section 6.6.

\subsection{Coherent propagation of white X-rays in a planar waveguide}

In this section, the far-field diffraction pattern of a directly-coupled planar waveguide supporting two guided modes is measured as a function of photon energy $E$. The waveguide is illuminated by an unfocused collimated white X-ray beam and the far-field diffraction patterns corresponding to different photon energy are simultaneously measured using an energy-dispersive semiconductor detector. The shape of the far-field diffraction pattern depends on the phase difference between the two guided modes at the exit of the waveguide. The dependence of this phase difference on the photon energy leads to a characteristic variation of the far-field diffraction pattern, namely oscillations in the energy spectra with a periodicity in $E^{-1}$, confirming coherent propagation of two guided modes.

The experiment thus (i) verifies the predicted properties of the guided modes, (ii) allows for an experimental verification of calculated individual propagation constants, (iii) shows that these modes superpose coherently, and (iv) clearly demonstrates that the electric field downstream of the waveguide is significantly different from that expected behind a hypothetical small slit of the same size.

In addition to these fundamental aspects of X-ray wave propagation in waveguides, which can conveniently be addressed by considering the energy dependence of the far-field diffraction pattern, the presence of a white beam is also motivated by possible improvements for future experiments. Increasing the energy bandwidth (e.g. using a full undulator harmonic with $\Delta E / E \approx 0.01$ ) can boost the number of photons available for experiments. This advantage is obvious for micro-fluorescence experiments, but monochromaticity can also be relaxed for imaging in the "direct contrast" regime. Therefore, it is important to investigate simultaneous propagation of different photon energies in the same waveguide.

Assuming a coherent incident X-ray beam, the electric field in a multi-mode waveguide is given as a superposition of multiple guided modes propagating with their individual propagation constants. Since the waveguide measured in this experiment supports two guided modes, $\psi_{0}$ and $\psi_{1}$, an incident plane wave may thus excite these two modes, propagating through the waveguide with their individual propagation constants $\beta_{0}$ and $\beta_{1}$. As illustrated in Section 3.2.1 and Fig. 3.4, differences between the propagation constants 
lead to the formation of a characteristic interference pattern inside the waveguide [FS05]. Although it does not seem feasible to measure the electric field directly, the field at the exit of a waveguide may be deduced from the far-field diffraction pattern. In particular, the shape of the electric field at the exit of the waveguide and the corresponding far-field diffraction pattern depend on the phase difference between the individual modes. For the $\psi_{0}$ and $\psi_{1}$ modes of the present waveguide, this phase difference is given by

$$
\Delta \phi_{01}=\left(\beta_{0}-\beta_{1}\right) \ell
$$

where $\ell$ denotes the length of the waveguide and $\beta_{0}$ and $\beta$ are the propagation constants of the guided modes. From Eq. 2.17) we find $\beta_{m}{ }^{2}=n_{1}{ }^{2} k^{2}-\kappa_{m}{ }^{2}$. Since $n_{1} k \gg \kappa_{m}$ we approximate $\beta_{m} \approx n_{1} k-\kappa_{m}^{2} / 2 n_{1} k$ and with $n_{1} \approx 1$ we find

$$
\beta_{0}-\beta_{1} \approx \frac{\kappa_{1}^{2}-\kappa_{0}^{2}}{2 k}
$$

Away from absorption edges, we can use Eq. (2.3) to express the decrement $\delta$ of the refractive index as a function of the electron density $\varrho_{e}$, and the waveguide parameter $V$ may be written as

$$
V \approx \sqrt{2 \delta_{2}-2 \delta_{1}} k d=2 \sqrt{\pi r_{e} \Delta \varrho_{e}} d
$$

where $\Delta \varrho_{e}=\varrho_{e, 2}-\varrho_{e, 1}$ denotes the difference between the electron densities in the cladding and in the guiding layer. Note that this expression is independent of photon energy. Since the number of guided modes as well as the values of the $\kappa_{m} d$ and $\gamma_{m} d$ are uniquely determined by $V$ [Mar74, Yeh90], the number of guided modes and the corresponding eigen functions are independent of photon energy as well. Accordingly, the $\kappa_{m}$ do not depend on $k$. Hence, the difference between the propagation constants given in Eq. (6.2) and so the phase difference at the exit of the waveguide is inversely proportional to $k$ or to the photon energy $E$.

The far-field diffraction pattern is given by

$$
I\left(k_{f}\right) \propto\left|\int \psi(\ell, z) \exp \left(i k_{f} z\right) d z\right|^{2}
$$

where $k_{f}=k \sin \alpha_{f}$ is the $z$ component of the wave vector (Fig 6.5). Ignoring absorption, we find

$$
I\left(k_{f}\right) \propto\left|c_{0} \tilde{\psi}_{0}\left(k_{f}\right)+c_{1} \exp \left[-i\left(\beta_{1}-\beta_{0}\right) \ell\right] \tilde{\psi}_{1}\left(k_{f}\right)\right|^{2}
$$

where the tilde denotes the Fourier transform. Assuming that the coefficients $c_{m}$ do not strongly depend on photon energy, the far-field diffraction pattern (measured as a function of $k_{f}$ ) is approximately the same for each two energies where the difference in $\Delta \phi_{01}$ is an integer multiple of $2 \pi$. Since $\Delta \phi_{01}$ is proportional to $E^{-1}$, the far-field diffraction pattern is periodic in $E^{-1}$ with a period

$$
\Delta_{E^{-1}}=\frac{8 \pi^{2}}{\left(\kappa_{1}^{2}-\kappa_{0}^{2}\right) \ell h c} .
$$




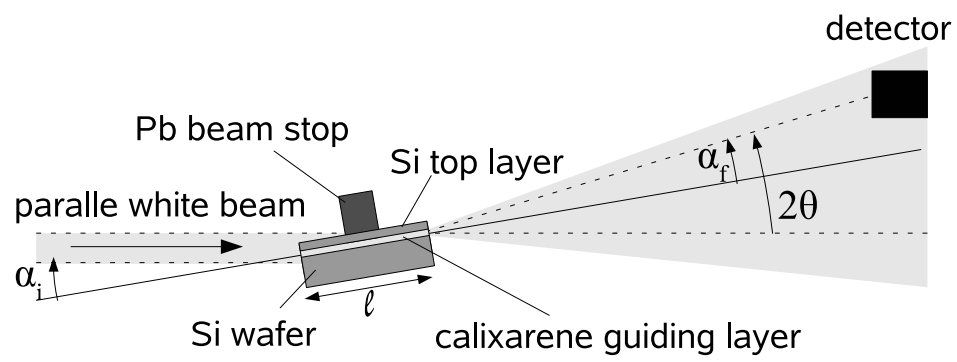

Figure 6.5: Experiment setup: The X-ray beam is coupled directly into the front-side of the waveguide. The intensity distribution in the far-field is measured for different incident angles $\alpha_{i}$. Depending on the photon energy, the maximum of the intensity pattern is found above or below $\alpha_{f}=0$. Distances and angles are not to scale.

Here, $h$ is Planck's constant and $c$ is the speed of light. Since the values of $\kappa_{0} d$ and $\kappa_{1} d$ are uniquely determined by $V$, the period $\Delta_{E^{-1}}$ only depends on $V, d$ and $\ell$.

Depending on the phase difference between the guided modes at the exit of the waveguide, the maximum of the far-field intensity distribution oscillates between positive and negative exit angles $\alpha_{f}$, unless $\alpha_{i}$ is exactly zero. Accordingly, the spectrum measured at a fixed exit angle shows characteristic oscillations with photon energy $E$.

\subsubsection{Experiment}

The experiment was carried out at the energy dispersive reflectometry (EDR) white beam dipole beamline at BESSY II $\left[\mathrm{PLG}^{+} 03\right]$. The dimensions of the incident beam were set by horizontal and vertical slits at $200 \times 20 \mu \mathrm{m}^{2}$. The Si drift chamber detector (Röntec XFlash 1000) has an energy resolution of approximately $200 \mathrm{eV}$ and was placed $1.04 \mathrm{~m}$ away from the waveguide. The detector aperture was $50 \mu \mathrm{m}$ in vertical direction, corresponding to an angular resolution of $\approx 0.003^{\circ}$ in $2 \theta$ (Fig.6.5).

The X-ray waveguide consists of a calixarene guiding layer in a Si cladding. The thickness of the calixarene layer was determined as $57 \mathrm{~nm}$ by X-ray reflectivity. Two samples of the same waveguide structure were measured with length $\ell$ of $(1.77 \pm 0.05) \mathrm{mm}$ and $(4.1 \pm 0.1) \mathrm{mm}$. Assuming densities of $2.33 \mathrm{~g} / \mathrm{cm}^{3}$ for Si and $1.18 \mathrm{~g} / \mathrm{cm}^{3}$ for calixarene $\left(\mathrm{C}_{60} \mathrm{H}_{60} \mathrm{O}_{12}\right)$, we find $V \approx 2 \pi$ and thus the waveguide supports two guided modes*. The corresponding mode constants are $\kappa_{0} d \approx 2.37$ and $\kappa_{1} d \approx 4.63$.

*The exact value $V \approx 6.294$, which is slightly larger than $2 \pi$, implies that there are 3 guided modes. However, in this case the $\psi_{2}$ mode is very close to cut-off. Consequently, a large portion of power is transmitted in the cladding and the $\psi_{2}$ mode is subject to significantly higher absorption than the other guided modes. Furthermore, for small $\alpha_{i}$ the value of the overlap integral in Eq. 2.28) is also very small. So we may ignore the $\psi_{2}$ contributions. 

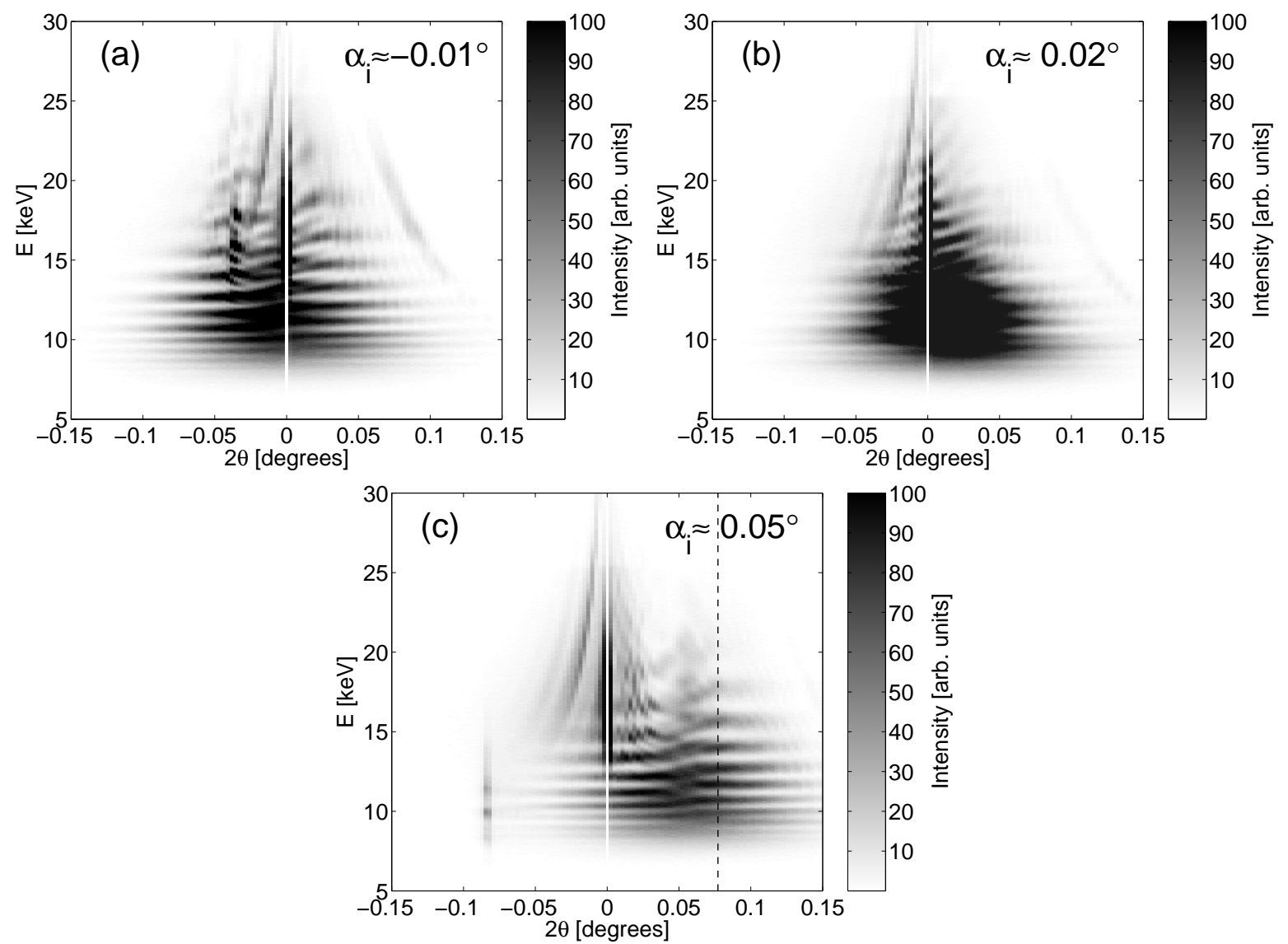

Figure 6.6: Far-field diffraction patterns measured behind the short waveguide $(\ell \approx 1.8 \mathrm{~mm})$ for varying angle of incidence $\alpha_{i}$. For $2 \theta=0$ no spectra could be measured due to the high intensity of the transmitted beam. The dashed line in (c) indicates the spectrum plotted in Fig. 6.8 (a).

\subsubsection{Results}

Fig. 6.6 and Fig. 6.7 show far-field diffraction patterns measured for different angles of incidence $\alpha_{i}$. The angle of incidence was increased in steps of $0.03^{\circ}$ and $0.02^{\circ}$, respectively. The absolute values of $\alpha_{i}$ are estimated from the centres of the diffraction patterns expected at $2 \theta=-\alpha_{i}$. Alternatively, the absolute values could be derived from the positions of the specularly reflected beams visible in Fig. 6.6(a) and Fig. 6.7(a). However, this gives slightly different values, which is probably due to interference of the reflected beam by the waveguide far-field.

At low photon energy there is practically no intensity transmitted, due to strong absorption in both the $\mathrm{Si}$ and the guiding layer. For medium energy, between $\approx 10 \mathrm{keV}$ and $\approx 15 \mathrm{keV}$, we get a nearly undisturbed waveguide far-field in agreement with the expression given in Eq. 6.4. The centre of the diffraction pattern moves in $2 \theta$ with $\alpha_{i}$, and the total intensity decreases with increasing $\left|\alpha_{i}\right|$. Along the energy axis, the angular position of the maximum oscillates between positive and negative $\alpha_{f}$ values. These oscillations are also clearly visible in spectra measured at fixed exit angles, as shown in Fig. 6.8. Plotted over $E^{-1}$, these spectra yield periods of $3.22 \times 10^{-3} \mathrm{keV}^{-1}$ for the 

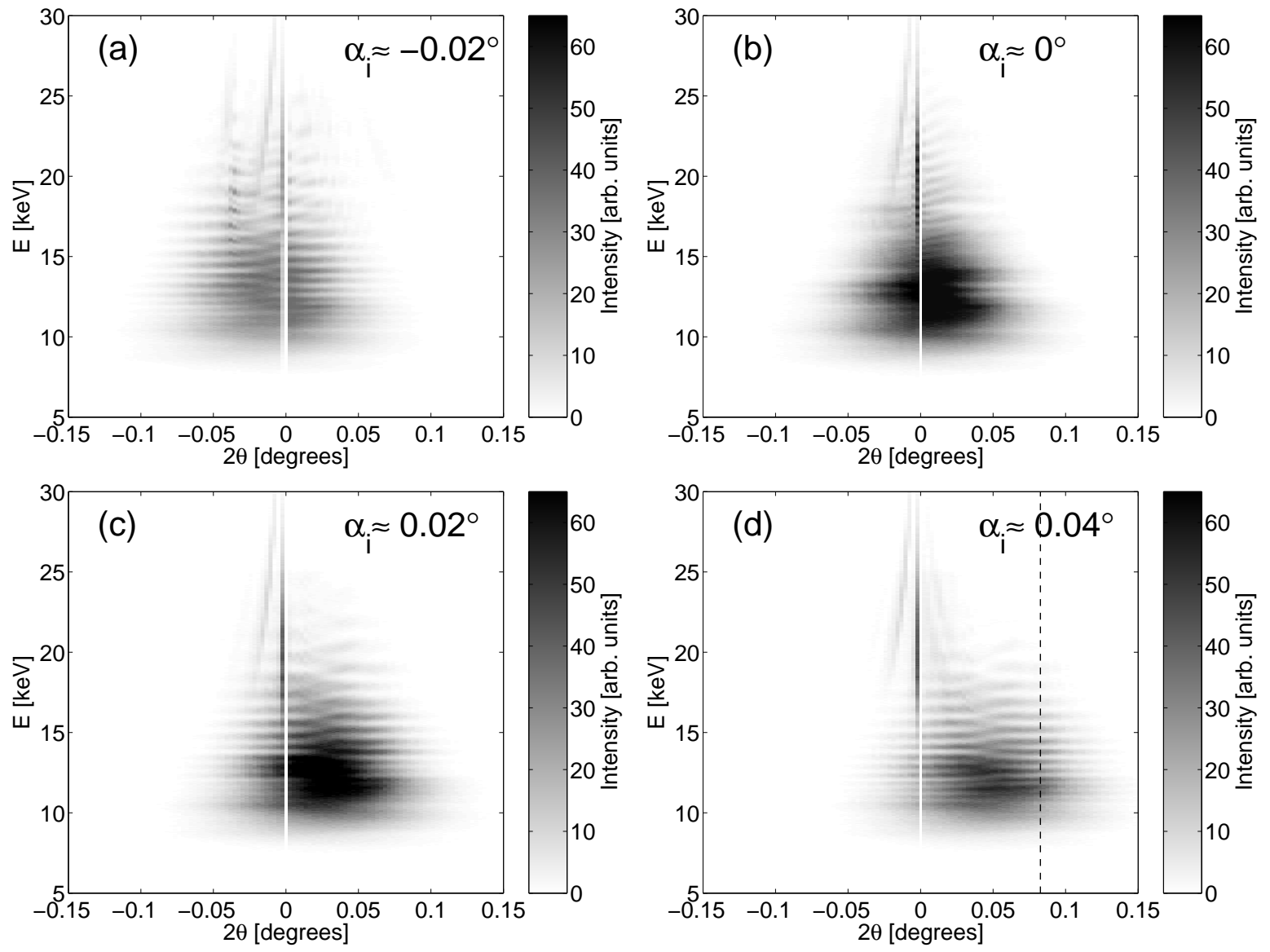

Figure 6.7: Far-field diffraction patterns measured behind the long waveguide $(\ell \approx 4.1 \mathrm{~mm})$ for varying angle of incidence $\alpha_{i}$. For $2 \theta=0$ no spectra could be measured due to the high intensity of the transmitted beam. The dashed line in (d) indicates the spectrum plotted in Fig. 6.8(b). 

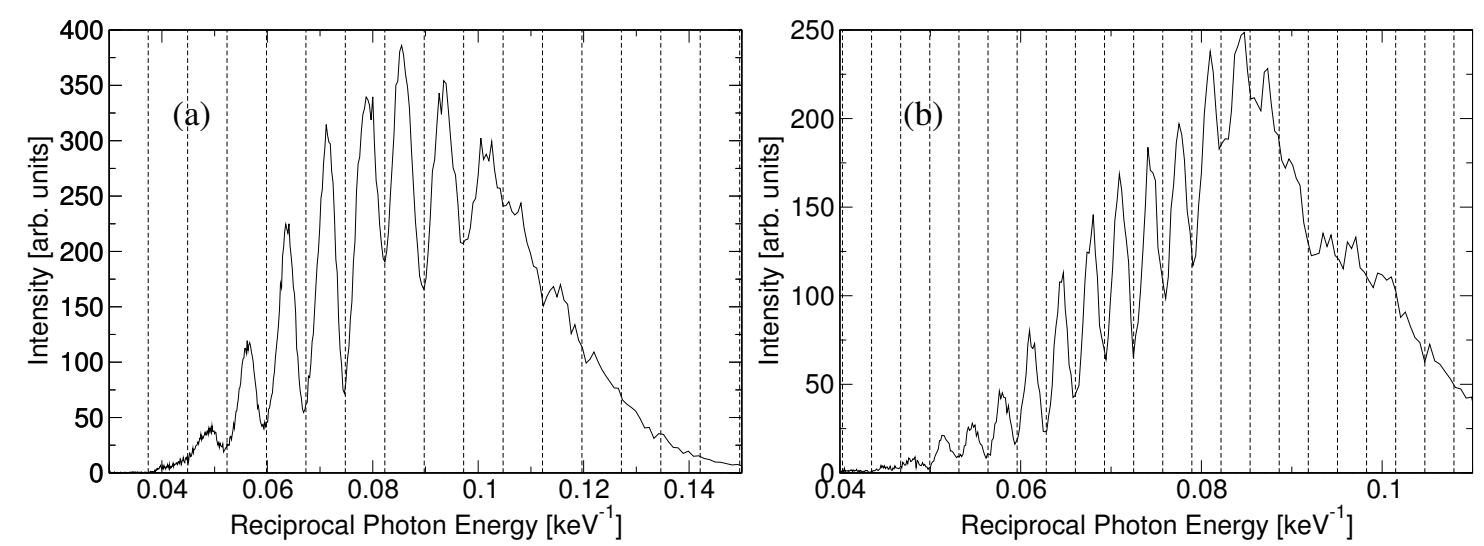

Figure 6.8: Spectra measured at a fixed exit angle $\alpha_{f}$ in the far-fields of the short (a) and the long (b) waveguide show characteristic oscillations equidistantly spaced in $E^{-1}$. This spacing decreases with an increasing waveguide length. A local maximum in the spectrum corresponds to an energy for which the maximum of the far-field diffraction pattern appears at $\alpha_{f}>0$, while local minima in the spectrum correspond to energies for which the maximum of the far-field diffraction pattern appears at $\alpha_{f}<0$. As indicated by the dashed lines, the measurements yield periods of $\Delta_{E^{-1}}=7.48 \times 10^{-3} \mathrm{keV}^{-1}$ (a) and $\Delta_{E^{-1}}=3.22 \times 10^{-3} \mathrm{keV}^{-1}$ (b), respectively.

short waveguide and $7.48 \times 10^{-3} \mathrm{keV}^{-1}$ for the longer one. From Eq. 6.6 we expect $\Delta_{E^{-1}}=(3.2 \pm 0.1) \times 10^{-3} \mathrm{keV}^{-1}$ and $\Delta_{E^{-1}}=(7.4 \pm 0.2) \times 10^{-3} \mathrm{keV}^{-1}$, respectively, in agreement with the experimental results. When $\alpha_{i}$ is very close to zero, these oscillations are hardly visible. This is due to the fact that for $\alpha_{i}=0$, the overlap integral in Eq. 2.28 vanishes for all odd modes and thus the $\psi_{1}$ mode is not excited. Accordingly, the far-field diffraction pattern measured for very small $\left|\alpha_{i}\right|$ is a slightly disturbed pattern of the $\psi_{0}$ mode.

At very high photon energy, absorption of the Si cladding decreases significantly and the waveguide far-field is superposed by transmitted and specularly reflected beams, accompanied by other disturbing contributions that are probably related to diffraction of these beams. The intense high energy components in the transmitted beam also made it impossible to measure spectra at $2 \theta=0$. Note that the Si cladding is nearly transparent at high photon energy (for the short waveguide, already at $E=15 \mathrm{keV}$ transmission is $2 \%$ ). Since the width of the transmitted beam is much smaller than the width of the waveguide far-field, only one line is missing in the patterns shown in Fig. 6.6 and Fig. 6.7. However, intensity of the waveguided beam itself decreases corresponding to a decrease in the spectrum of the bending magnet source $\left[\mathrm{PLG}^{+} 03\right]$.

Apart from verifying the theory of mode propagation, the experiment also shows the energy range within which the waveguide is suited for applications. At the lower end, significant absorption in the waveguide damps the far-field intensity, while at the upper end the far-field diffraction pattern is disturbed by radiation transmitted through the cladding. Since both ends are determined by absorption, the applicative energy range can be adapted to higher or lower photon energy by increasing or decreasing the length 
of the waveguide. Note that the shorter waveguide provides significantly higher intensity in particular at low photon energy near $10 \mathrm{keV}$, while the far-field of the long waveguide shows less disturbing contributions of radiation transmitted through the cladding.

\subsubsection{Conclusions}

The experiment verifies the results of field calculations and demonstrates the energy range within which the waveguides may be applied to provide a small and coherent beam with sufficient intensity and low background. Furthermore, the characteristic oscillations in the far-field diffraction patterns for different photon energy $E$ indicate that the electric field in the waveguides is indeed given by a coherent superposition of guided modes. The characteristic frequency in $E^{-1}$ is in agreement with the propagation constants calculated from the analytical theory of planar waveguides highlighted in Section 2.3.1. Finally, the experiment clearly demonstrates that the electromagnetic field at the exit of a planar $\mathrm{X}$-ray waveguide and in the far-field may be very different from that expected behind a hypothetical small slit of the same size.

\subsection{Direct coupling into two-dimensionally confining waveguides}

In this section we demonstrate direct coupling of a pre-focused beam into two-dimensionally confining waveguides. As a result of the small cross-sectional dimensions of the guiding core, a high density of flux provided by the pre-focusing device is even more crucial than in the case of planar waveguides. The aim is to provide an undisturbed waveguide beam with sufficient flux for applications like lens-less imaging.

\subsubsection{Experiment}

A first proof-of-principle experiment was carried out at the ID22 undulator beamline of the European Synchrotron Radiation Facility. The experiment setup was the same as described in Section 6.4. At $12.5 \mathrm{keV}$ photon energy the KB mirrors provided a flux of $3.3 \times 10^{11} \mathrm{ph} / \mathrm{s}$ in a focal spot of $2.5 \times 3.8 \mu \mathrm{m}^{2}$.

\subsubsection{Results}

A two-dimensionally confining waveguide with cross-sectional dimensions of $30 \times 70 \mathrm{~nm}^{2}$ (Fig. 6.9(a) was aligned in the focal spot of the KB mirrors. The waveguide consists of a PMMA guiding core in $\mathrm{Si}$ with a length $\ell=4.1 \mathrm{~mm}$. Flux density in the focal spot corresponds to a flux of $7.3 \times 10^{7} \mathrm{ph} / \mathrm{s}$ hitting the front face of the waveguide. 


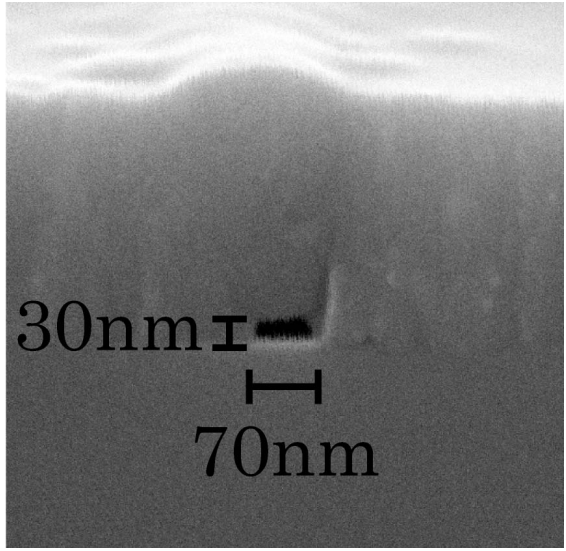

(a) Scanning electron microscopy image.

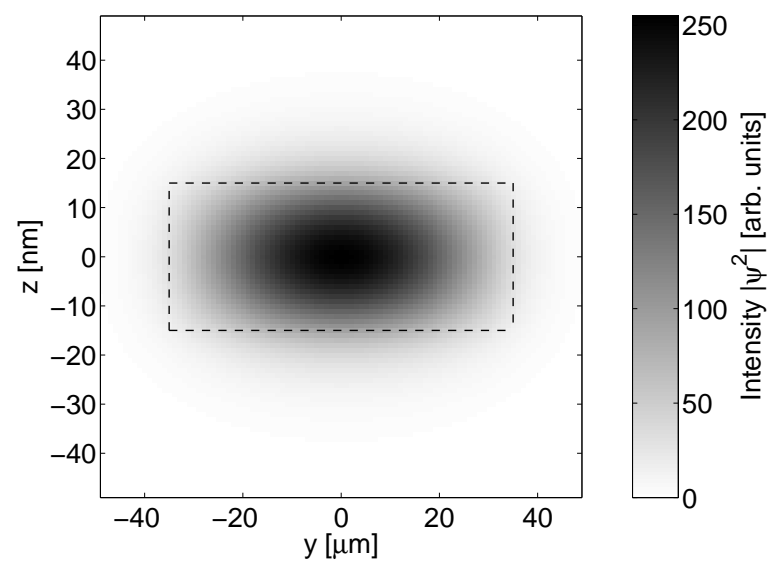

(b) Calculated electric field intensity at the end face of the waveguide.

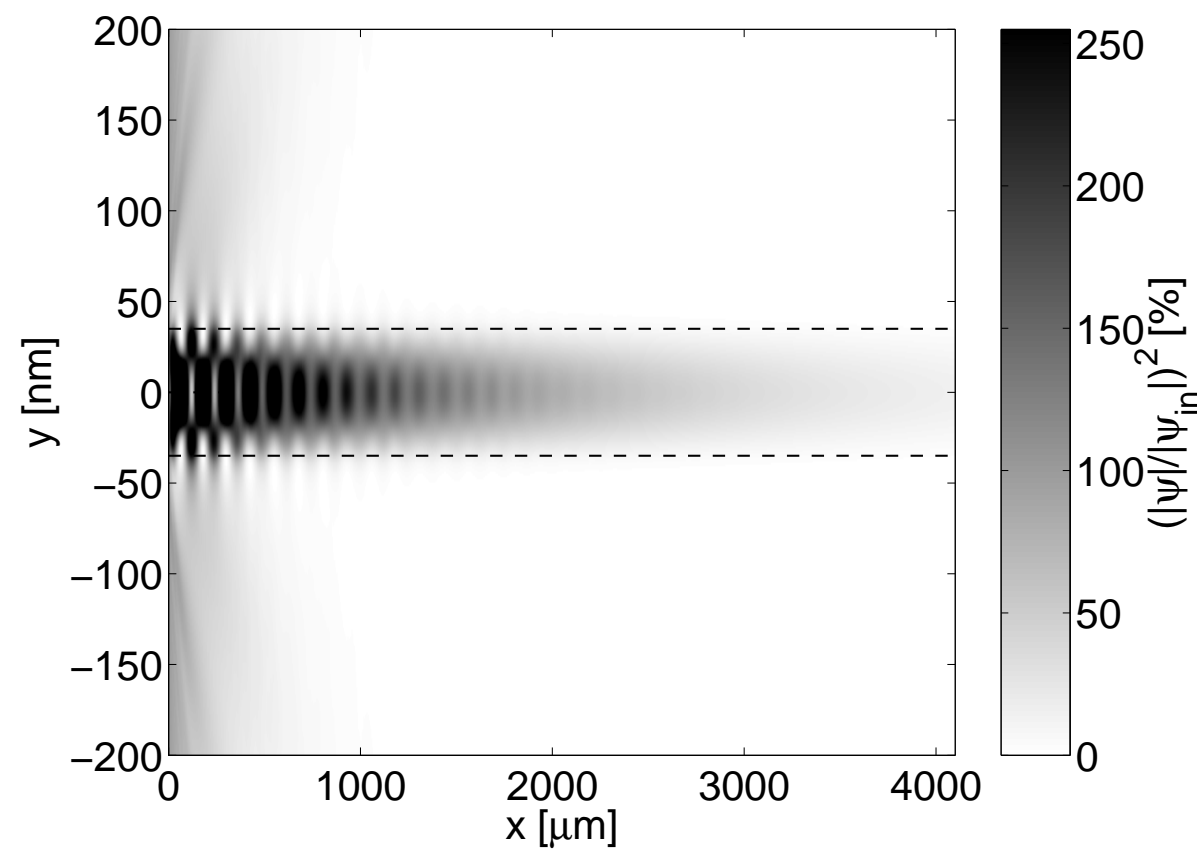

(c) Calculated field intensity along the optical axis inside the waveguide.

Figure 6.9: A scanning electron microscopy image (a) illustrates the small cross-sectional dimensions of the guiding core $\left(\approx 70 \times 30 \mathrm{~nm}^{2}\right.$ PMMA in Si). A finite-difference calculation predicts that the width (full width at half maximum) of the beam exiting the waveguide is significantly smaller than the guiding core cross-section dimensions (b). Intensity decreases significantly along the optical axis due to absorption (c) and the calculation yields transmission of $\approx 7 \%$. The calculation is carried for $12.5 \mathrm{keV}$ photon energy and dashed lines indicate the interfaces of the guiding core. 


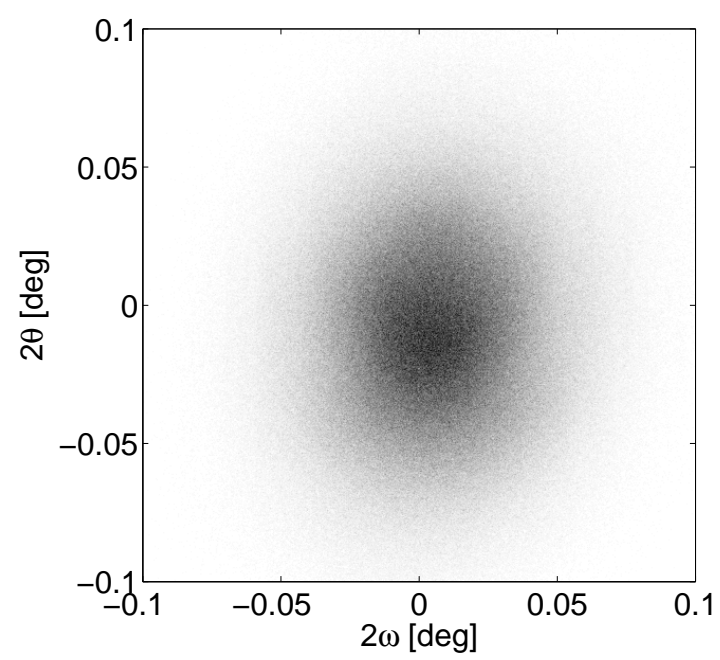

Figure 6.10: Far-field diffraction pattern of a two-dimensionally confining waveguide. The waveguide consists of a calixarene guiding core with cross-sectional dimensions of $\approx 100 \times 80 \mathrm{~nm}^{2}$ in $\mathrm{Si}$. The length of the waveguide is $4 \mathrm{~mm}$ and photon energy is $10.4 \mathrm{keV}$.

Downstream of the waveguide, flux was $3.5 \times 10^{6} \mathrm{ph} / \mathrm{s}$, corresponding to a transmission of $\approx 5 \%$. A finite difference calculation of the electric field inside the waveguide (Fig. 6.9(b) and Fig. 6.9(c) shows that the electric field at end face of the waveguide is dominated by the fundamental guided mode. Higher modes are damped out and transmission is $\approx 7 \%$. Taking into account the limited angular acceptance of the waveguide and the divergence of the pre-focused beam, this approximately agrees with the measurement. The beam size at the end face of the waveguide, i. e. the full width at half maximum of the electric field intensity, is determined by the shape of the fundamental mode. The calculation thus predicts a beam size of $\approx 25 \times 47 \mathrm{~nm}^{2}$, which is significantly below the cross-sectional dimensions of the guiding core.

This specific experiment is described in more detail in $\left[\mathrm{JFO}^{+} 05\right]$. Several more waveguides have been used at that beamline in direct-coupling. Typical flux obtained from the waveguides was $10^{6}-10^{7} \mathrm{ph} / \mathrm{s}$, depending on the parameters of the respective samples.

Besides sufficient flux, imaging experiment also require a homogeneous and undisturbed intensity distribution in the waveguide beam. Fig. 6.10 shows a measured far-field diffraction pattern demonstrating that a divergent beam with a very homogeneous intensity distribution is indeed obtained.

Since the density of flux in the focus of the KB optics is extremely high [STMC $\left.{ }^{+} 05\right]$, beam damage becomes an important issue. Indeed, significant changes are observed in the far-field diffraction pattern when the waveguides are exposed to the KB beam for a long time (see Fig. 6.11). Evidently, these changes may significantly disturb imaging experiments. Because of the limited availability of beamtime, the temporal evolution of the far-field diffraction pattern has not been studied in more detail. However, beam damage typically occurred after some hours of exposure in the focus of the KB mirrors. 


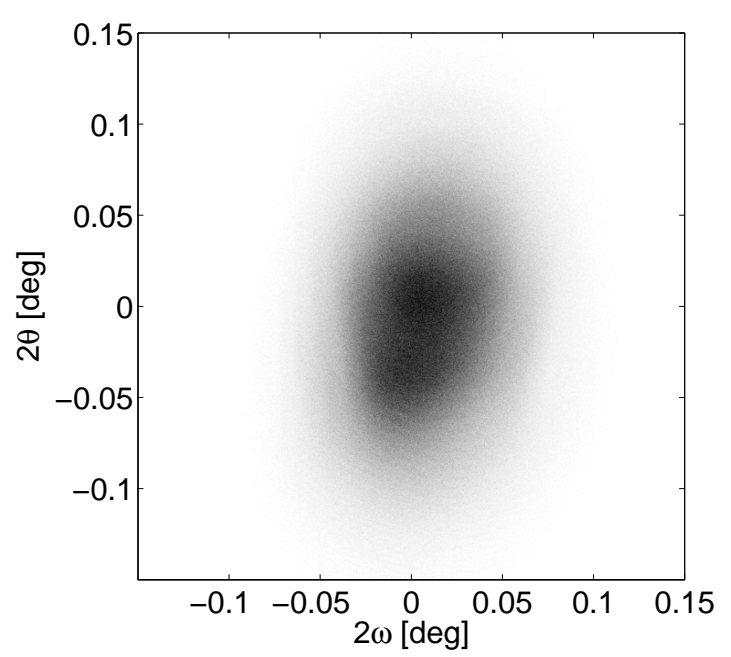

(a) New waveguide.

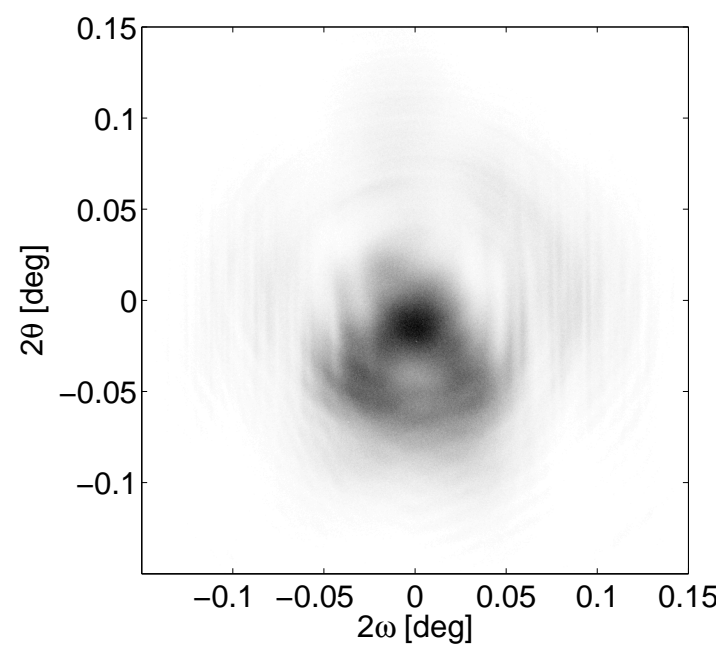

(b) Used waveguide.

Figure 6.11: The far-field diffraction pattern of two-dimensionally confining waveguides significantly changes when the waveguide is exposed to the focus of the KB optics for many hours. Evidently, the beam obtained from a new waveguide (a) is much better suited for imaging experiments than the beam obtained from a waveguide used for many hours (b). Both images were recorded with the same waveguide during the same experiment. (Calixarene guiding core in Si, photon energy $10.4 \mathrm{keV}$.)

\subsubsection{Conclusions}

By direct coupling of a pre-focused undulator beam, monochromatic flux of $10^{6}-10^{7} \mathrm{ph} / \mathrm{s}$ was obtained from two-dimensionally confining waveguides. The cross-sectional dimensions of the waveguides may be as small as $30 \mathrm{~nm}$ and the recorded far-field diffraction patterns indicate that the obtained beams are well suited for applications in waveguide-based X-ray microscopy, in particular for lens-less imaging.

\subsection{Waveguide Gratings}

In order to characterise two-dimensionally confining waveguides without the need for high flux density provided by focused undulator beams, arrays of many identically prepared waveguides can be characterised in the unfocused parallel beam of a bending magnet beamline.

Ignoring coupling between the individual waveguides [ST91] and assuming a perfect lattice of identical waveguides illuminated by a plane wave, the electric field at the exit of the waveguides is given as a convolution of the grating and the field of an individual two-dimensionally confining waveguide. Consequently, intensity in the far-field diffraction pattern is given by a grating diffraction pattern $I_{\text {grating }}$ enveloped by the far-field diffraction 


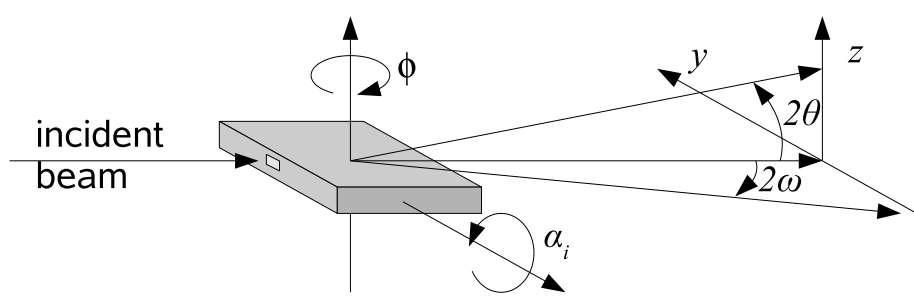

Figure 6.12: Notation of angles. $\alpha_{i}$ and $\phi$ denote rotations of the waveguide while $2 \theta$ and $2 \omega$ denote the corresponding angles of the detector position.

pattern of a single waveguide $I_{\mathrm{wg}}$ [Pfe02] (see Fig. 6.12 concerning the notation):

$$
I(2 \omega, 2 \theta) \propto I_{\mathrm{wg}}(2 \omega, 2 \theta) \times I_{\text {grating }}(2 \omega) .
$$

Assuming coherent illumination of $N$ waveguides spaced by a period $\Lambda$, the grating pattern is given by $\operatorname{Vog} 97$

$$
I_{\text {grating }}(2 \omega) \propto \frac{\sin ^{2}[N \pi \Lambda \sin (2 \omega) / \lambda]}{\sin ^{2}[\pi \Lambda \sin (2 \omega) / \lambda]} .
$$

However, in real experiments the lattice peaks are further broadened due to (i) the finite coherence length of the incident radiation, (ii) the finite detector resolution, and (iii) imperfections of the waveguides [OFJS05].

\subsubsection{Experiment}

Experiments with waveguide gratings were carried out at the bending magnet beamline BM20 of the ESRF (Rossendorf beamline, ROBL). Photon energy was set to $12 \mathrm{keV}$ using a $\mathrm{Si}(111)$ double-crystal monochromator and higher harmonics were practically eliminated by vertically focusing Si mirrors. The waveguide far-field diffraction pattern was measured using a CCD detector (Bruker SMART, $60 \times 60 \mu \mathrm{m}^{2}$ pixel size) placed $3.23 \mathrm{~m}$ behind the waveguide and by scanning with a scintillation detector.

The measured gratings consist of calixarene guiding cores in Si. Length of the waveguides is $4.0 \mathrm{~mm}$ and the total width of the gratings is $300 \mu \mathrm{m}$. Before lithographic processing, the thickness of the calixarene layer was $\approx 80 \mathrm{~nm}$. The waveguides were arranged on gratings with periods $\Lambda$ of $150-200 \mathrm{~nm}$. A scanning electron microscopy image of the grating with a period of $150 \mathrm{~nm}$ is shown in Fig. 6.13.

\subsubsection{Results}

Fig. 6.14 shows the far-field diffraction patterns of gratings with periods of $150 \mathrm{~nm}$ and $200 \mathrm{~nm}$, measured using the CCD detector. The grating was arranged in a horizontal direction and the diffraction pattern accordingly shows grating peaks in this direction. A Fourier transformation of the measured intensity pattern yields the autocorrelation function of the electric field at the waveguide exit and the grating structure is clearly visible (Fig. 6.14, bottom). 


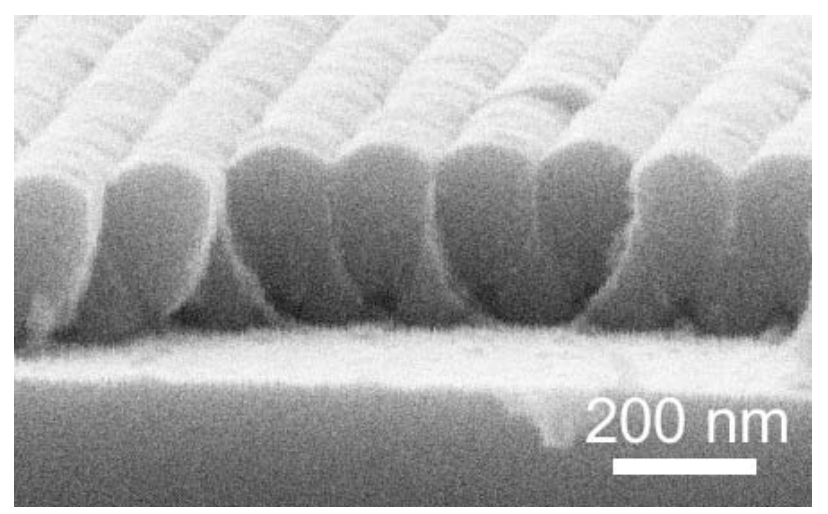

Figure 6.13: Scanning electron microscopy image of a waveguide grating with a period of $150 \mathrm{~nm}$.

When the horizontal angle of incidence $\phi$ is varied, the far-field envelope changes in the same way as the far-field diffraction pattern of a single waveguide (see Section 6.4). Although with multi-mode waveguides both shape and position are changed, the most intense grating peaks may be expected near $2 \omega=\phi$ and the total intensity decreases with an increasing absolute value of $\phi$. The positions of the grating peaks remain practically unchanged since the projection of the grating period only varies like $\cos \phi$. Fig. 6.15 shows the far-field diffraction patterns measured at different incident angles $\phi$. The far-field was scanned using a scintillation detector which was opened wide in vertical direction and thus integrates the intensity in this direction. Indeed, the scans show that total intensity decreases with increasing $|\phi|$. The envelope is approximately shifted by $\phi$ while the positions of the grating peaks do not change. Since the waveguide supports multiple modes, also the shape of the envelope changes significantly, which can be most clearly seen with $|\phi|=0.06^{\circ}$.

For illustration, Fig. 6.15(a) shows a calculated pattern. The waveguide far-field envelope is taken from a finite-difference calculation assuming a guiding core thickness of $60 \mathrm{~nm}$. The positions of the lattice peaks are calculated for a period of $150 \mathrm{~nm}$ and the shape of the peak is modelled by a Gaussian with a width adapted to the measured data.

\subsubsection{Conclusions}

Waveguide gratings allow the characterisation of two-dimensionally confining waveguides without the need for highly brilliant sources. This is of high practical importance, in particular for testing of new waveguide preparation techniques or material combinations.

Evidently, the experiments allow the measuring of average transmission of the waveguides. But also divergence of the exiting beam may be extracted from the envelope of the far-field diffraction pattern, which is given by the far-field diffraction pattern of an individual waveguide. Furthermore, the autocorrelation function of the electric field at the exit of the waveguides may be calculated from the measured far-field diffraction pattern of the waveguide grating. 

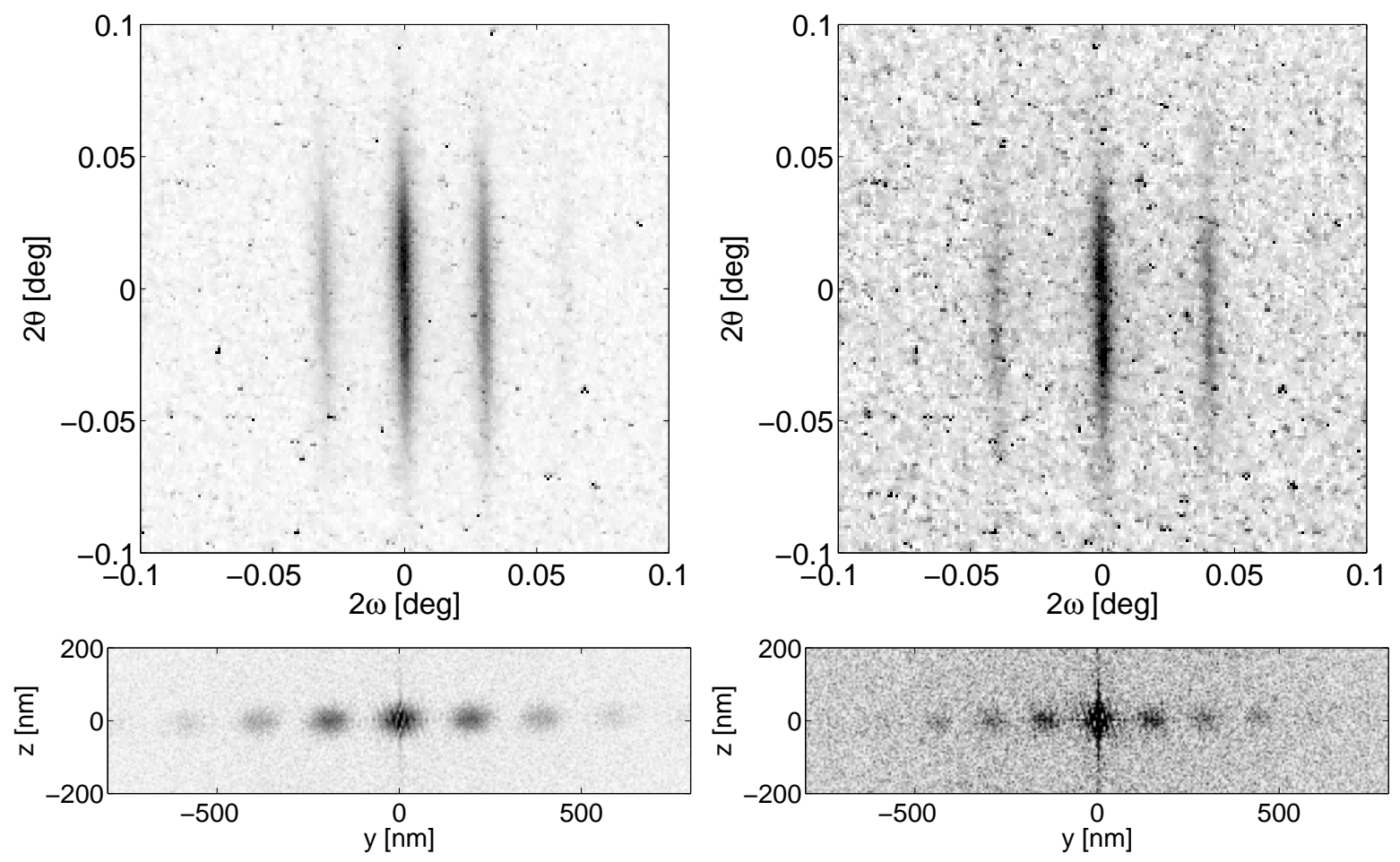

Figure 6.14: Far-field diffraction patterns of waveguide gratings with periods of $200 \mathrm{~nm}$ (left) and $150 \mathrm{~nm}$ (right) measured at $E=12 \mathrm{keV}$. The lower row shows the modulus of the autocorrelation functions of the electric field obtained by a Fourier transformation of the far-field pattern. The different grating periods are clearly visible.

\subsection{Tapered waveguides}

In a tapered waveguide, the diameter of the guiding core decreases in propagation direction (Fig. 6.16). The waveguide thus compresses the beam and may provide a higher density of flux at the exit. For example, a gain of about six was obtained by Zwanenburg et al., who used a planar waveguide with a tunable air gap $\left[\mathrm{ZBP}^{+} 00\right]$. Significantly higher gain of up to a factor of 1,000 was reported for tapered glass capillaries [BHT94, HTB94]. However, a tapered waveguide may not compress an arbitrarily large beam. This is easily understood by ray optics, as shown in Fig. 6.17, since multiple reflections increase the grazing angle between the X-ray beam and the interface. When the grazing angle becomes larger than the critical angle of total reflection, the beam is decoupled into the cladding.

It was shown by Pfeiffer et al. that X-rays may be reflected on the edges of twodimensional nanostructures [PSD02] and we will now consider tapered two-dimensionally confining waveguides.

\subsubsection{Experiment}

Experiments with tapered waveguides were carried out at the bending magnet beamline BM20 of the ESRF using the same experimental setup as described in Section 6.7.1. The 


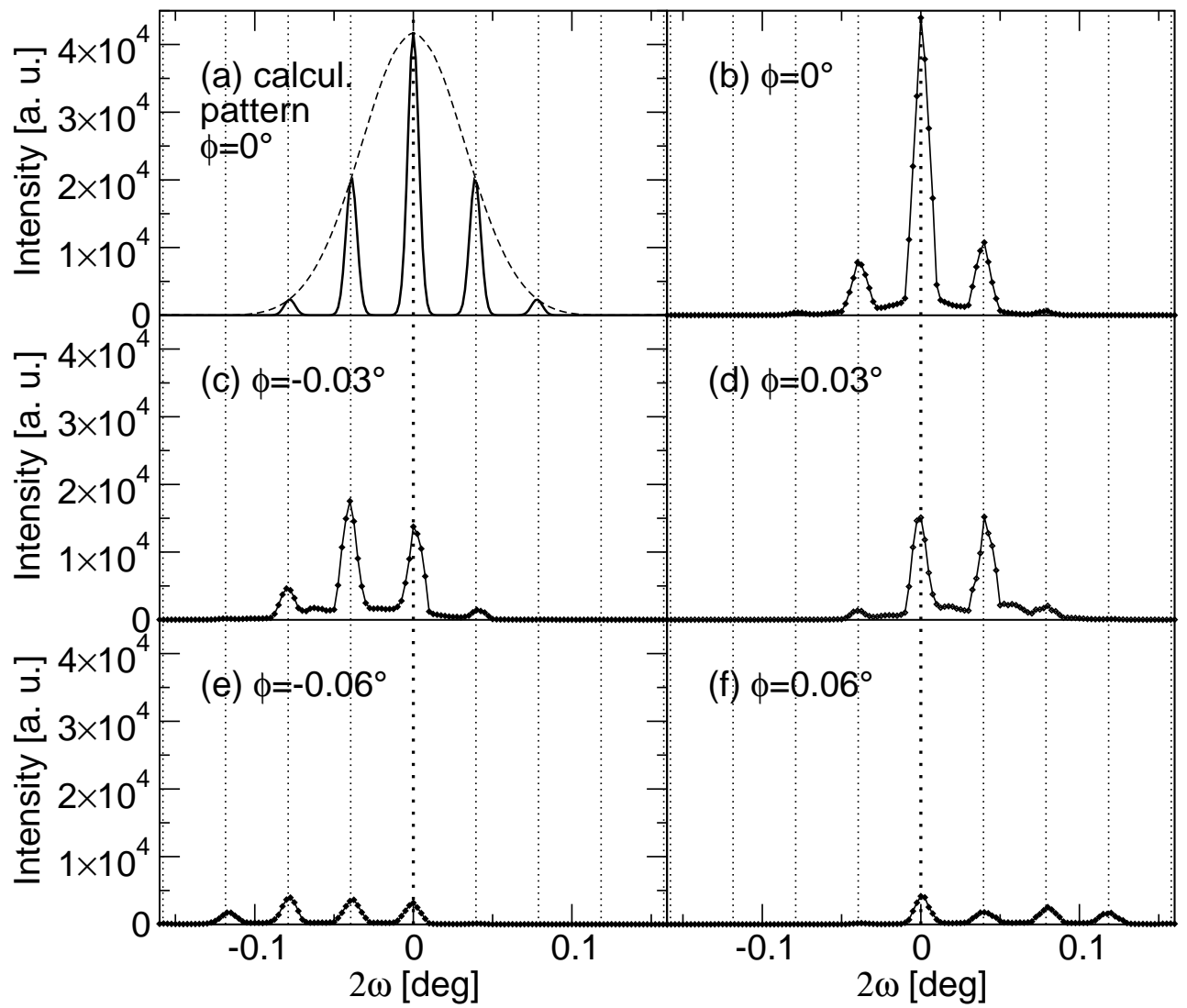

Figure 6.15: Calculated (a) and measured (b-f) horizontal far-field scans of a waveguide grating with varying horizontal angle of incidence $\phi$ (calixarene/Si waveguides, period $150 \mathrm{~nm}$, $E=12 \mathrm{keV}$ ). The dotted lines indicate the calculated positions of the grating peaks. The dashed line in (a) shows the enveloping diffraction pattern of an individual waveguide calculated for a guiding core thickness of $60 \mathrm{~nm}$ by finite-difference calculations. The grating peaks of the calculated curve were assumed to have a Gaussian shape and the width was adapted to the measured curves.

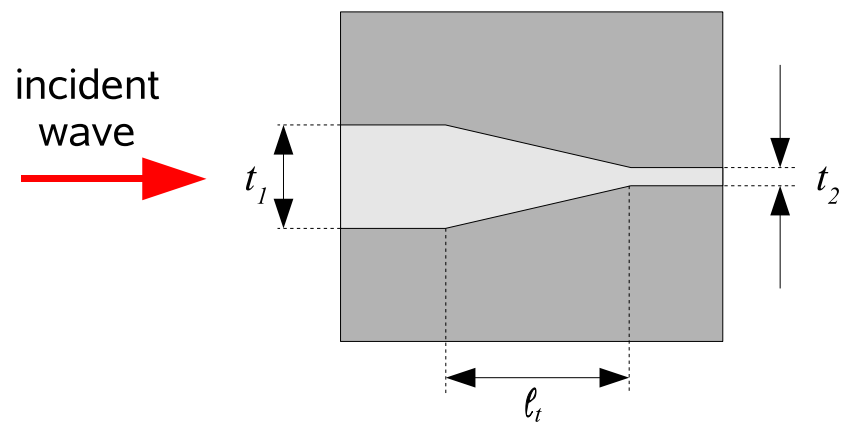

Figure 6.16: (a) In a tapered waveguide the guiding core dimensions decrease from $t_{1}$ to $t_{2}$ over a distance $\ell_{t}$. 


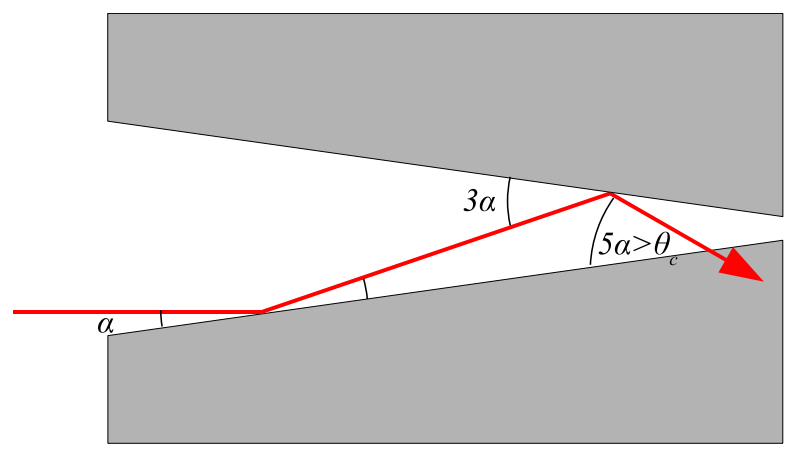

Figure 6.17: The grazing angle between an X-ray beam reflected in a tapered waveguide and the interface increases with the number of reflections. When the grazing angle becomes larger than the critical angle of total reflection $\theta_{c}$, the beam is decoupled from the guiding core.

width $t_{2}$ at the exit of the waveguide is $\approx 60 \mathrm{~nm}$ and the width $t_{1}$ at the front face is varied from 60 to $800 \mathrm{~nm}$. To enable a characterisation at a bending magnet beamline, we have measured gratings of up to 200 individual waveguides. The length of the waveguides is $4.0 \mathrm{~mm}$ and the length $\ell_{t}$ of the tapered region is $3.0 \mathrm{~mm}$. In front of and behind the tapered region the waveguides are parallel to ensure that a small variation in total length does not change the parameters $t_{1}$ and $t_{2}$ (see Fig. 6.16).

\subsubsection{Results}

Fig. 6.18 shows translational scans through arrays of tapered waveguides at $\phi=0$. The entrance width $t_{1}$ of the tapered waveguides is $200 \mathrm{~nm}, 400 \mathrm{~nm}$ and $800 \mathrm{~nm}$, respectively. As summarised in the table shown in Fig. 6.18, the flux per individual waveguide increases with an increasing entrance width $t_{1}$. For $t_{1}=800 \mathrm{~nm}$ the tapered waveguides provides a flux nearly six times greater than the non-tapered waveguides. At the same time, the angular acceptance of the waveguides decreases with an increasing entrance width (Fig. 6.19). For comparison, the angular acceptance and the transmission, i. e. intensity gain, of tapered one-dimensionally confining waveguides was calculated by finite-difference calculations* The calculation illustrates that a larger waveguide entrance increases the gain but decreases the angular acceptance. The measured angular acceptance of the tapered waveguides is slightly broader than the calculated values, but the smaller acceptance for larger $t_{1}$ is clearly visible. The limited angular acceptance of tapered waveguides significantly reduces the gain when a pre-focusing optic is used.

\subsubsection{Conclusions}

It was shown that tapered two-dimensionally confining waveguides provide a higher density of flux than corresponding parallel waveguide. However, the gain in flux is accompanied

* Note that a calculation of these curves for two-dimensionally confining waveguides is very timeconsuming. The calculation of each data point then requires a field calculation in three dimensions. 

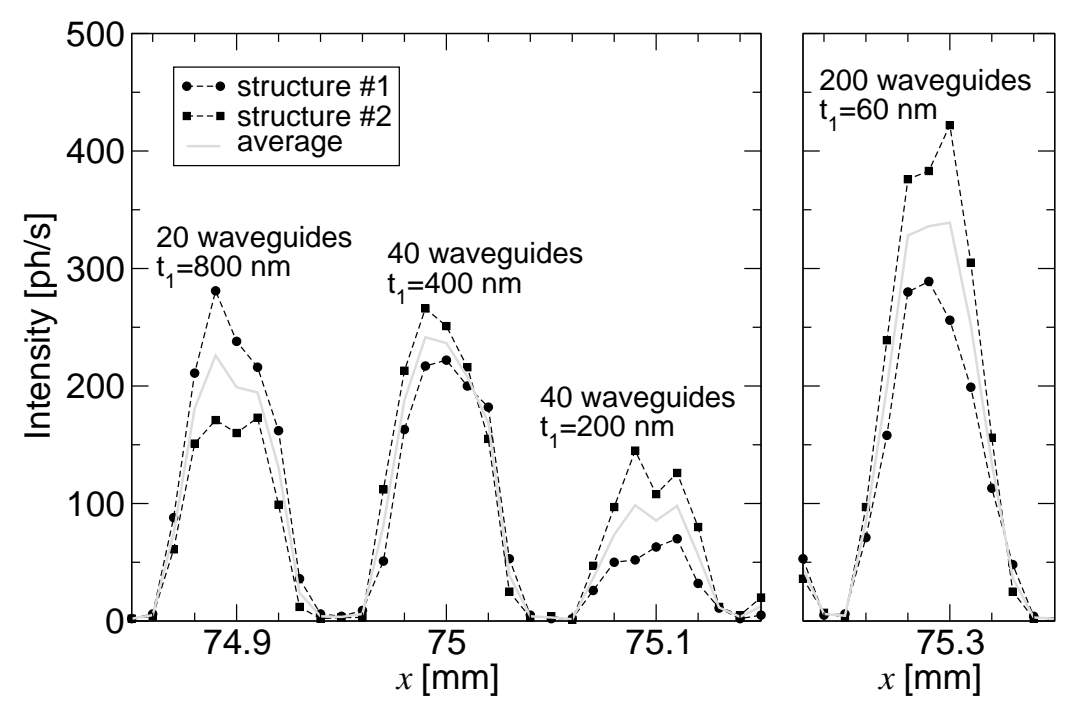

\begin{tabular}{r|c}
\multicolumn{1}{c|}{$t_{1}$} & \multicolumn{1}{l}{$\begin{array}{l}\text { average flux } \\
\text { per waveguide }\end{array}$} \\
\hline $60 \mathrm{~nm}$ & $(1.7 \pm 0.3) \mathrm{ph} / \mathrm{s}$ \\
$200 \mathrm{~nm}$ & $(2.4 \pm 0.9) \mathrm{ph} / \mathrm{s}$ \\
$400 \mathrm{~nm}$ & $(5.7 \pm 0.6) \mathrm{ph} / \mathrm{s}$ \\
$800 \mathrm{~nm}$ & $(10.3 \pm 2.2) \mathrm{ph} / \mathrm{s}$
\end{tabular}

Figure 6.18: Translational scans through arrays of tapered (left) and untapered (middle) waveguides with the indicated entrance width $t_{1}$ and an exit width of $\approx 60 \mathrm{~nm}$. The table on the right summarises the average intensities per individual waveguide.

by a smaller angular acceptance that may be below the typical beam divergence of prefocusing devices like the Kirkpatrick-Baez mirrors used for direct coupling in single twodimensionally confining waveguides. Thus, the optimum taper angle has to be adapted to a specific experiment.

\subsection{Curved waveguides and double waveguides}

In the applications of waveguides considered so far, the waveguide serves in principle as a small slit or pinhole. In this section, we consider "true guiding" of X-rays in the sense that the beam is guided to a location outside the primary beam or into a different direction. Thus, the front faces of two waveguides can be illuminated coherently while both position and emission direction of the end face may be varied.

Since analytical theory of losses in curved waveguides is quite complex and requires detailed knowledge of the eigen functions, this work is restricted to an experimental investigation. At first, we investigate losses in curved two-dimensionally confining waveguides. We then present $Y$ double waveguide as an experimental realisation of two coherent pointlike sources enabling coherent two-beam experiments like reference beam holography.

\subsubsection{Double waveguides}

In analogy to the results obtained from the waveguide gratings, one might expect that the far-field diffraction pattern of a double waveguide is given by a Young pattern [ST91] enveloped by the diffraction pattern of an individual waveguide. However, when the distance between two waveguides is small, their fields overlap and coupling between the two 

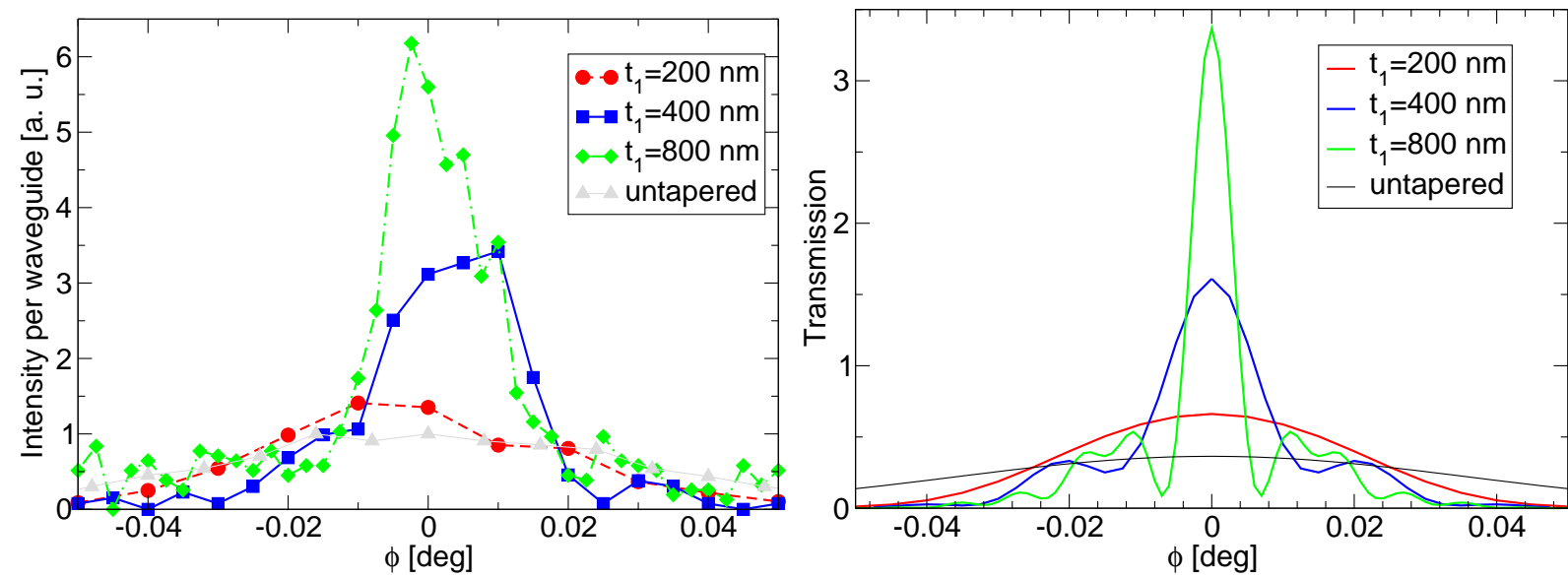

Figure 6.19: Measured angular acceptance of tapered two-dimensionally confining waveguides (left) and calculated $E=12 \mathrm{keV}, t_{2}=60 \mathrm{~nm}$ ) angular acceptance of comparably tapered onedimensionally confining waveguides (FD calculations, right). Only one sample of each $t_{1}$ value was measured, but the intensities are scaled with respect to the average flux obtained from both samples with the respective $t_{1}$ value given in Fig. 6.18.

waveguides occurs [ST91]. As illustrated in Fig. 6.20, intensity then oscillates between the two waveguides. There may even be certain positions where practically all intensity is concentrated in only one waveguide. The corresponding far-field diffraction pattern may then evidently not be given by a Young pattern.

Note that coupling also occurs in the waveguide gratings. But for reasons of symmetry, the field of the individual waveguides in an infinite grating illuminated by a plane wave may only differ in phase. In practice, only the outermost waveguides in a grating may show a significantly different field than the others. A detailed analysis of coupling between X-ray waveguides is beyond the scope of this thesis. However, the effect might influence some specific experiments. For example, it could influence the visibility of the Young fringes in the far-field diffraction pattern of the $\mathrm{Y}$ double waveguides presented in Section 6.9.3.

In the experiment, the far-field diffraction pattern was measured for a two-dimensionally confining double waveguide. The experiment setup was according to Section 6.4.1. Photon energy was $12 \mathrm{keV}$ and the diffraction pattern was measured using a CCD detector with a pixel size of $60 \times 60 \mu \mathrm{m}^{2}$ (Photonics Science XIOS II) placed $3.3 \mathrm{~m}$ downstream of the waveguide.

The individual waveguides are displaced by $100 \mathrm{~nm}$ and three patterns were measured for incidence angles $\phi$ of $0^{\circ},-0.03^{\circ}$, and $-0.06^{\circ}$. Although finite-difference calculations indicate that coupling between the waveguide is comparably weak (Fig. 6.21), the measured far-field diffraction patterns shown in Fig. 6.22 may indeed not be interpreted as a pattern of a single waveguide multiplied with a Young pattern for $\phi=-0.03^{\circ}$ and $\phi=-0.06^{\circ}$. These patterns thus clearly indicate coupling between the individual waveguides. However, due to symmetry reasons, there is no intensity oscillating between the waveguides for $\phi=0^{\circ}$; the measured pattern then is indeed enveloped by a Young pattern. 


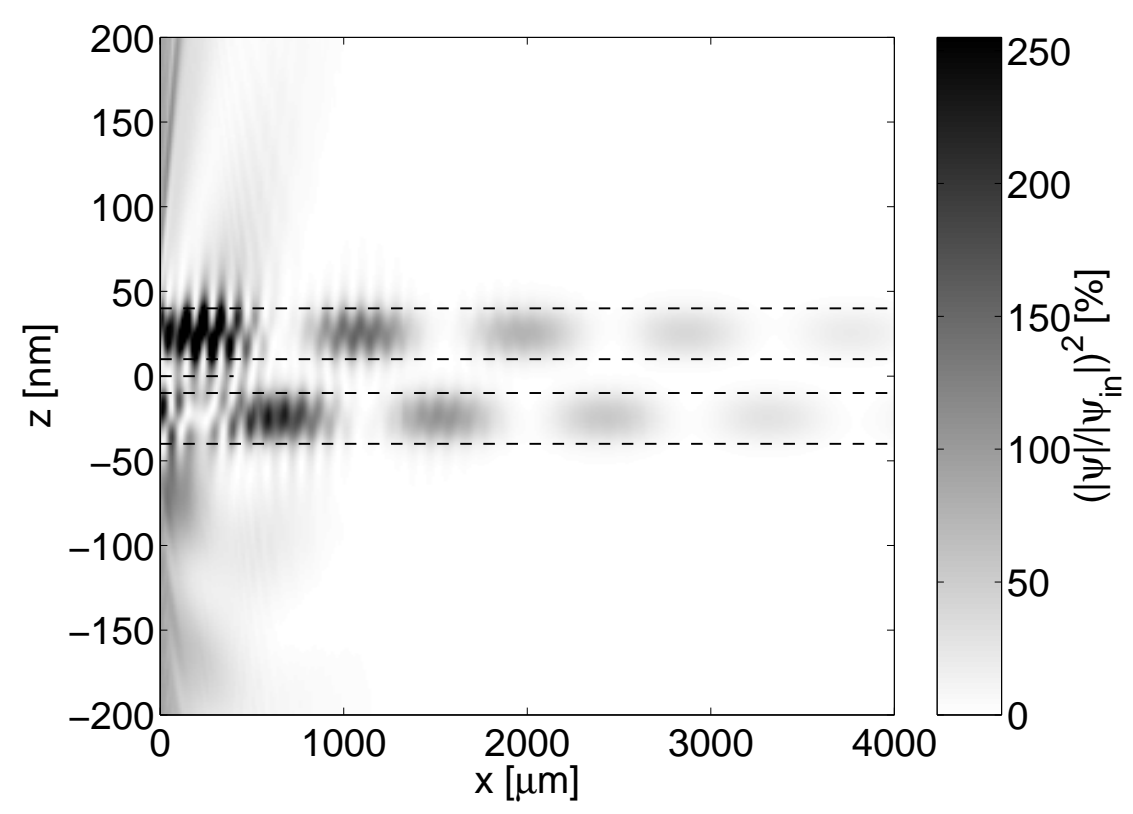

Figure 6.20: Coupling between two planar waveguides through an interlayer with a thickness of $20 \mathrm{~nm}$ (finite-difference calculation). The waveguides indicated by the dashed lines consist of calixarene guiding layers $\left(\mathrm{C}_{60} \mathrm{H}_{60} \mathrm{O}_{12}, \rho=1.175 \mathrm{~g} / \mathrm{cm}^{3}\right)$ with a thickness of $30 \mathrm{~nm}$ in $\mathrm{Si}$. The angle of incidence is $0.03^{\circ}$ and photon energy is $12 \mathrm{keV}$.

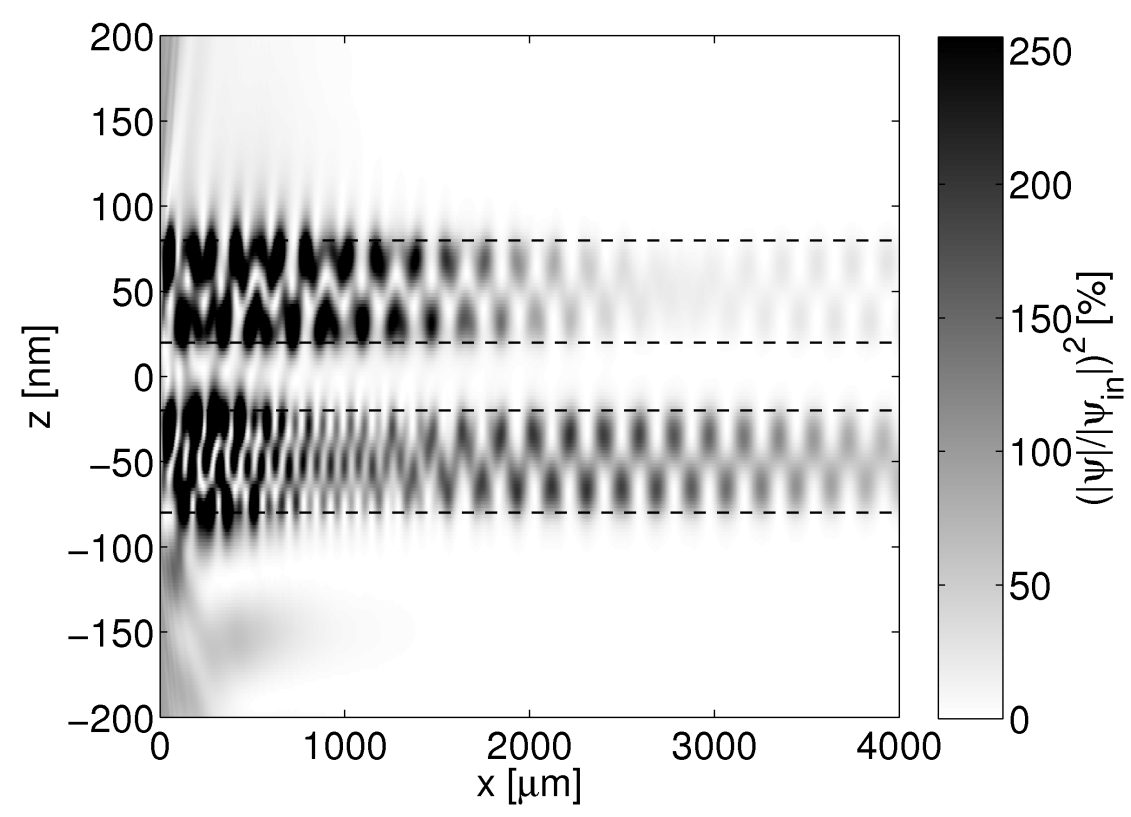

Figure 6.21: Coupling between two-dimensionally confining waveguides (two rectangular calixarene waveguides with cross-sectional dimensions of $60 \times 60 \mathrm{~nm}^{2}$ in Si displaced by $100 \mathrm{~nm}$ ). The angle of incidence is $0.04^{\circ}$ and photon energy is $12 \mathrm{keV}$. Intensity was obtained from finitedifference calculations and the figure shows intensity in a slice through the centres of the two waveguides. 


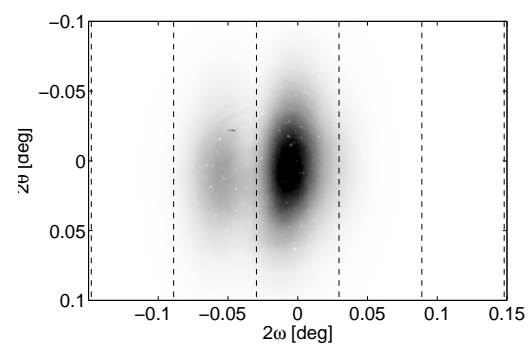

(a) $\phi \approx 0^{\circ}$.

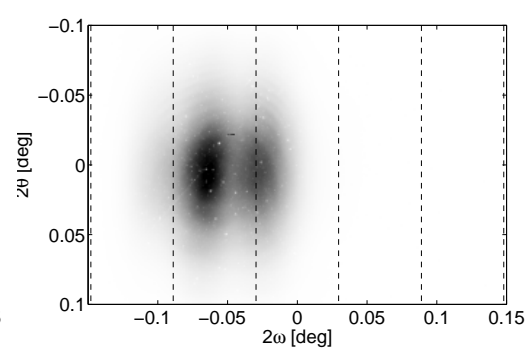

(b) $\phi \approx-0.03^{\circ}$.

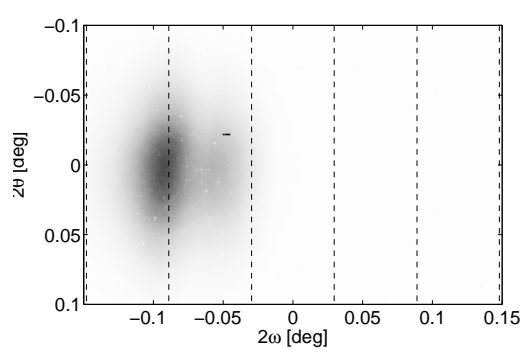

(c) $\phi \approx-0.06^{\circ}$.

Figure 6.22: The far-field diffraction pattern of two waveguides displaced by a small distance (in this instance $100 \mathrm{~nm}$ ) may not be described by the pattern of an individual waveguide multiplied by a Young pattern. The dashed lines indicate the positions where the corresponding Young pattern is zero. The measured far-field has nearly zero intensity at these positions only at $\phi=0$. (For the sake of visibility, intensity measured for $\phi \approx-0.06^{\circ}$ is scaled up by a factor of four compared to the other patterns.)

\subsubsection{Curved waveguides}

Curved waveguides were studied experimentally by measuring gratings of curved calixarene/Si waveguides. Each grating consists of 100 waveguides with dimensions of $\approx$ $60 \times 60 \mathrm{~nm}^{2}$ and the grating period is $\Lambda=150 \mathrm{~nm}$. The gratings were prepared with deflection angles $\alpha_{d}$ of $1^{\circ}, 2^{\circ}$, and $5^{\circ}$, respectively. As shown in Fig. 6.23, the waveguides are curved along a circular arc over a length $\ell_{d}$ of $3 \mathrm{~mm}$. Photon energy was $12 \mathrm{keV}$.

Fig. 6.24 shows the measured intensity detected in the far-field for deflection angles of $1^{\circ}$ and $2^{\circ}$. For $5^{\circ}$ no waveguide beam was detected, indicating that intensity is very low. Total intensity downstream of the grating was $\approx 55 \mathrm{ph} / \mathrm{s}$ for $1^{\circ}$ deflection and $\approx 11 \mathrm{ph} / \mathrm{s}$ for $2^{\circ}$ deflection. For comparison, two gratings with uncurved waveguides were measured. The uncurved waveguide consisted of 200 waveguides and maximum intensity in the far-field was $\approx 400$ and $\approx 280 \mathrm{ph} / \mathrm{s}$, respectively. Accordingly, a flux of $\approx 170 \mathrm{ph} / \mathrm{s}$ is expected for an uncurved grating with 100 waveguides. Flux behind the curved waveguides with a deflection angle of $1^{\circ}$ is thus decreased by a factor of about three compared to the uncurved waveguide. For a deflection angle of $5^{\circ}$ the factor is about 15 .

Note that two waveguides deflected by $1^{\circ}$ in opposite directions may already be separated by $35 \mu \mathrm{m}$ on a length of $1 \mathrm{~mm}$. The $\mathrm{Y}$ double waveguides introduced in the next section are separated by only up to $10 \mu \mathrm{m}$ on a length of $3 \mathrm{~mm}$; we may thus expect only low curvature losses for these structures. 


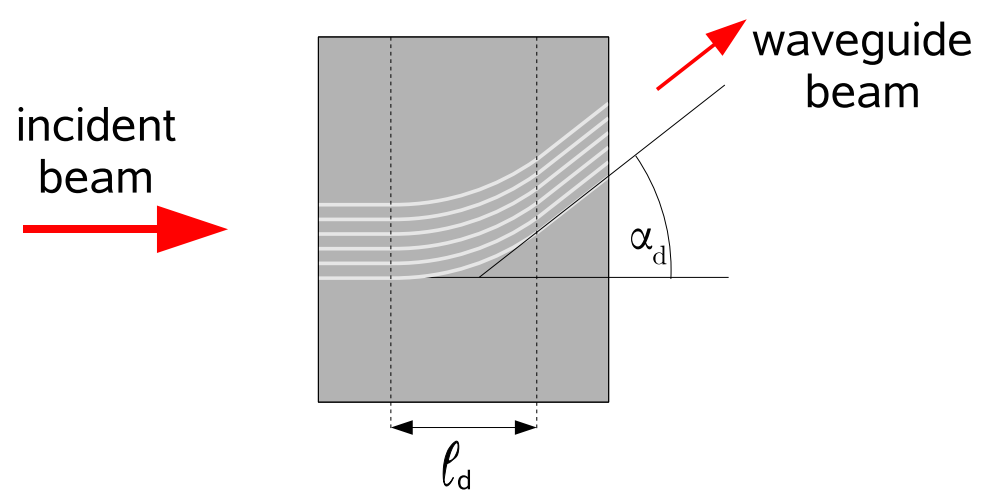

Figure 6.23: Sketch of the curved waveguide gratings. At the beginning and at the end the waveguides are straight. In between, the waveguides are curved on a length $\ell_{d}$ along a circular arc to a deflection angle $\alpha_{d}$. Lengths and angles are not to scale.

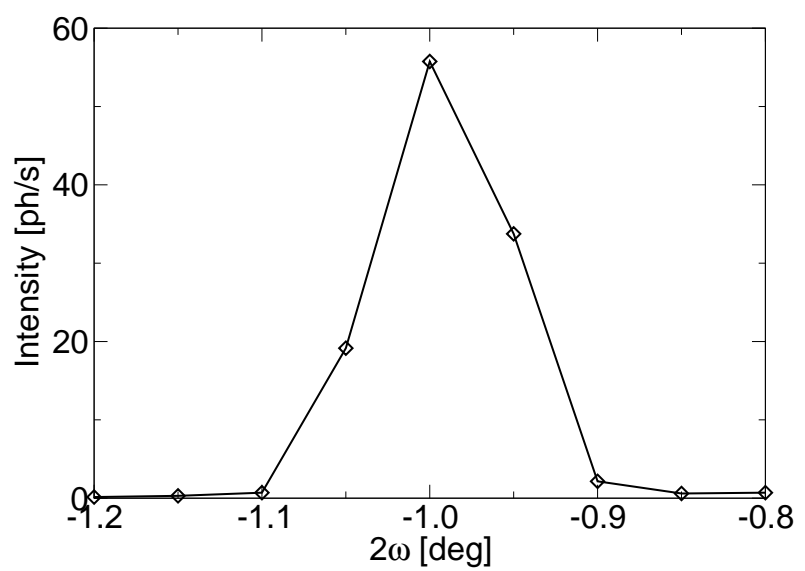

(a) $\alpha_{c}=-1^{\circ}$.

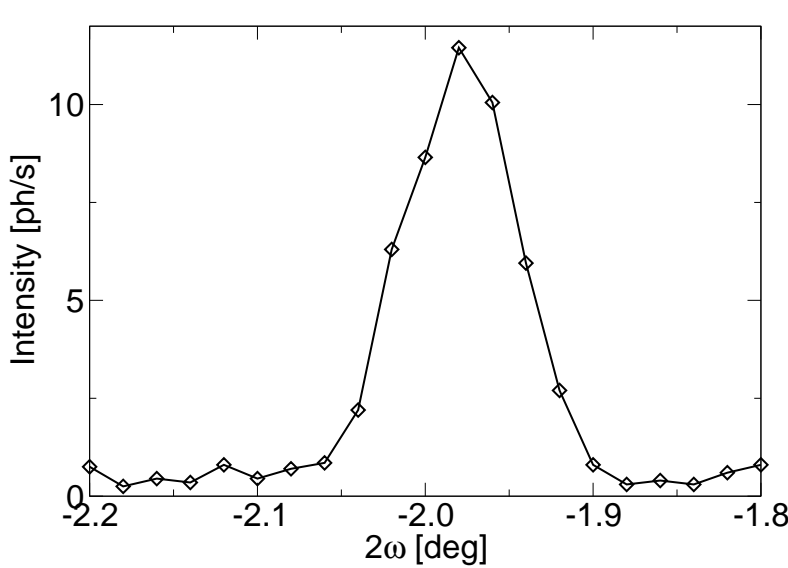

(b) $\alpha_{c}=-2^{\circ}$.

Figure 6.24: Intensity pattern measured behind arrays of curved waveguides. The waveguides deflect the beam by $1^{\circ}$ and $2^{\circ}$, respectively, in negative direction (see Fig. 6.12). The waveguides are curved along a circular arc on a length $\ell_{d}$ of $3 \mathrm{~mm}$. 


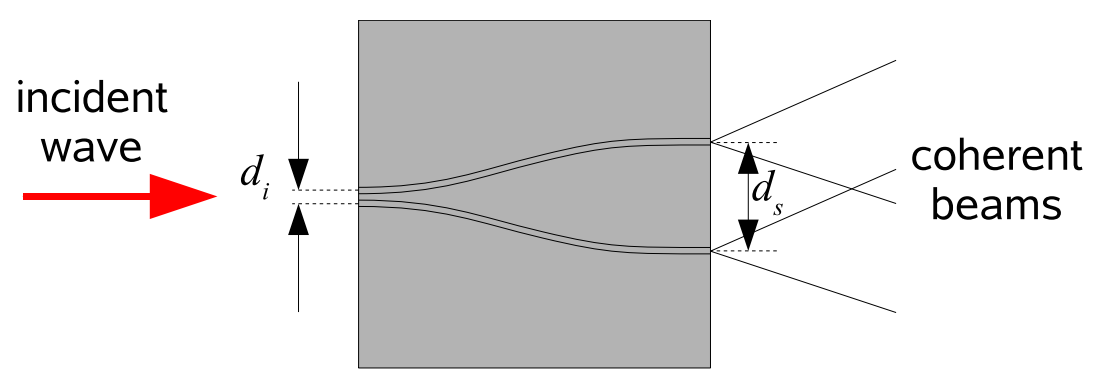

Figure 6.25: A Y double waveguide may provide two coherent beams for off-axis holography, provided that the spacing $d_{i}$ of the individual waveguides at the entrance is not larger than the transverse coherence length of the incident beam. The distance $d_{s}$ between the two waveguide sources then determines the maximum field of view.

\subsubsection{Y double waveguides}

Waveguide-based off-axis holography requires two coherently illuminated waveguides. Since the spacing $d_{s}$ of the waveguide end faces limits the accessible field of view, this distance should be large compared to the cross-sectional dimensions of the individual waveguides. On the other end, the distance $d_{i}$ between the waveguide front faces has to be small compared to the transverse coherence length $\xi_{t}$ of the incident beam. Typically, the transverse coherence length is significantly smaller than the desired field of view. However, both requirements may be fulfilled using appropriately curved $\mathrm{Y}$ double waveguides, as sketched in Fig. 6.25. Assuming an incoherent source of size $\sigma$ at a distance $L$, the transverse coherence length is given by ANM01

$$
\xi_{t}=\frac{\lambda}{2} \frac{L}{\sigma}
$$

To obtain sufficient flux, the waveguides are placed in the focal spot of two perpendicularlycrossed Kirkpatrick-Baez mirrors. Considering the mirrors as an incoherent source with the distance given by the focal distance $f$ and the size given by the size of the beam incident on the mirrors, we may calculate the corresponding transverse coherence length. Since the waveguides are spaced in horizontal direction, we have to use the values of the horizontally focusing mirror which are $f=0.19 \mathrm{~m}$ and an incident horizontal beam size of $\approx 100 \mu \mathrm{m}$. The corresponding transverse coherence length at $10.4 \mathrm{keV}$ is $\approx 100 \mathrm{~nm}$. The waveguides were accordingly prepared with $d_{i}=100 \mathrm{~nm}$. The detected far-field diffraction pattern corresponds to the pattern of a single waveguide multiplied with the Young interference pattern of a double-slit with distance $d_{s}$. The angular spacing between the Young fringes is given by

$$
\alpha_{Y} \approx \sin \alpha_{Y}=\lambda / d_{s} .
$$

Since these fringes have to be resolved by the detector, the angular resolution of the detector limits the maximum distance $d_{s}$ that may be used in off-axis holography experiments. In the present experiment, a CCD detector with a pixel size of $20 \times 20 \mu^{2}$ (Princeton Instruments PI-SX:1300) was placed at a distance of $3.00 \mathrm{~m}$ (limited by the dimensions 
of the experimental hutch) downstream of the waveguide. This corresponds to an angular resolution of $\approx 7 \mu \mathrm{rad}$. Demanding at least 2 pixels per fringe, we find a maximum distance of $d_{s} \approx 9 \mu \mathrm{m}$ at a photon energy of $10.4 \mathrm{keV}$.

Fig. 6.26 shows far-field diffraction patterns measured for Y double waveguides with $d_{s}$ in the range $3-10 \mu \mathrm{m}$. For $d_{s}=3 \mu \mathrm{m}$ and $d_{s}=5 \mu \mathrm{m}$ the Young fringes are clearly resolved and even for $d_{s}=10 \mu \mathrm{m}$ they are still visible, although spatial resolution of the CCD detector is evidently a problem.

In conclusion, the measured far-field diffraction patterns indicate that the $\mathrm{Y}$ double waveguides indeed provide two coherent beams. The application of these devices in offaxis holography is shown in Section 7.7 .

\subsubsection{Conclusions}

The prepared waveguides allow X-rays to be guided in a direction that may differ from the direction of the primary beam by up to $2^{\circ}$. However, this is accompanied by significant losses in flux increasing strongly with the angle of deflection.

No significant losses were measured for very small deflection angles. The presented Y double waveguides allow the separation of two coherently illuminated waveguides by up to $10 \mu \mathrm{m}$, and it was shown that the two beams exiting the waveguides superpose coherently. The maximum distance of separation was however not limited by the waveguides but by the resolution of the CCD detector. This suggests an application of the Y double waveguides to off-axis holography where one waveguide illuminates the sample and the other waveguide provides the reference wave. 
(a) $d_{s}=3 \mu \mathrm{m}$
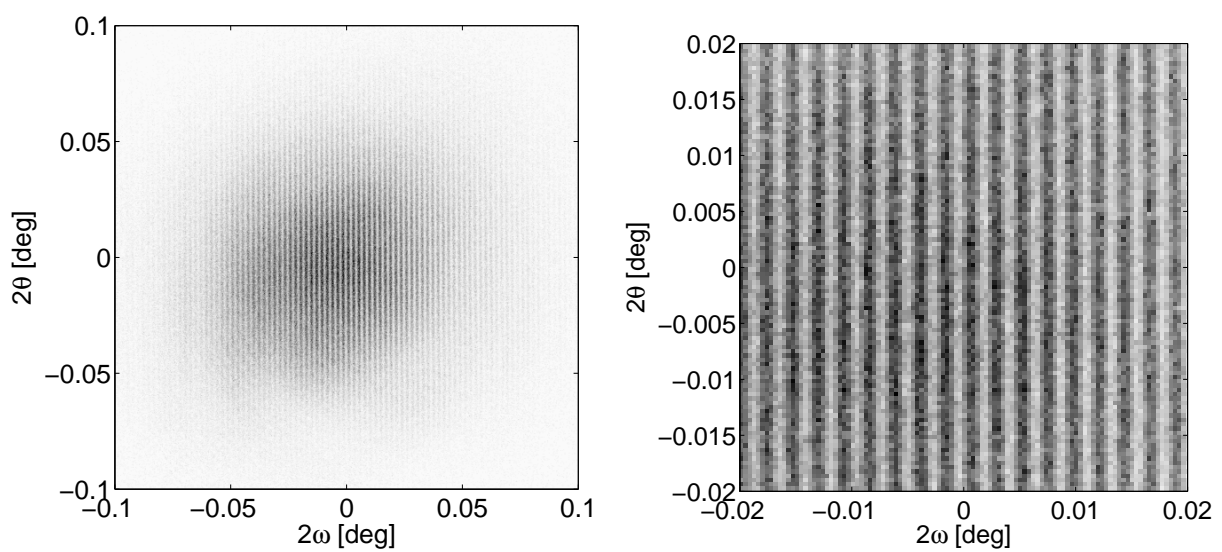

(b) $d_{s}=5 \mu \mathrm{m}$.
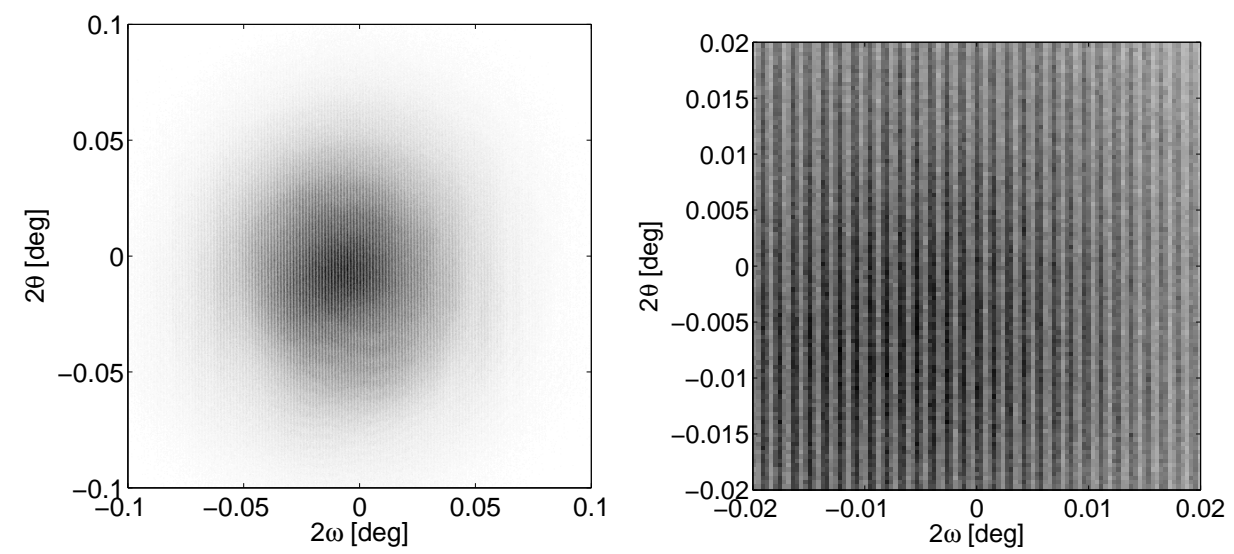

(c) $d_{s}=10 \mu \mathrm{m}$.
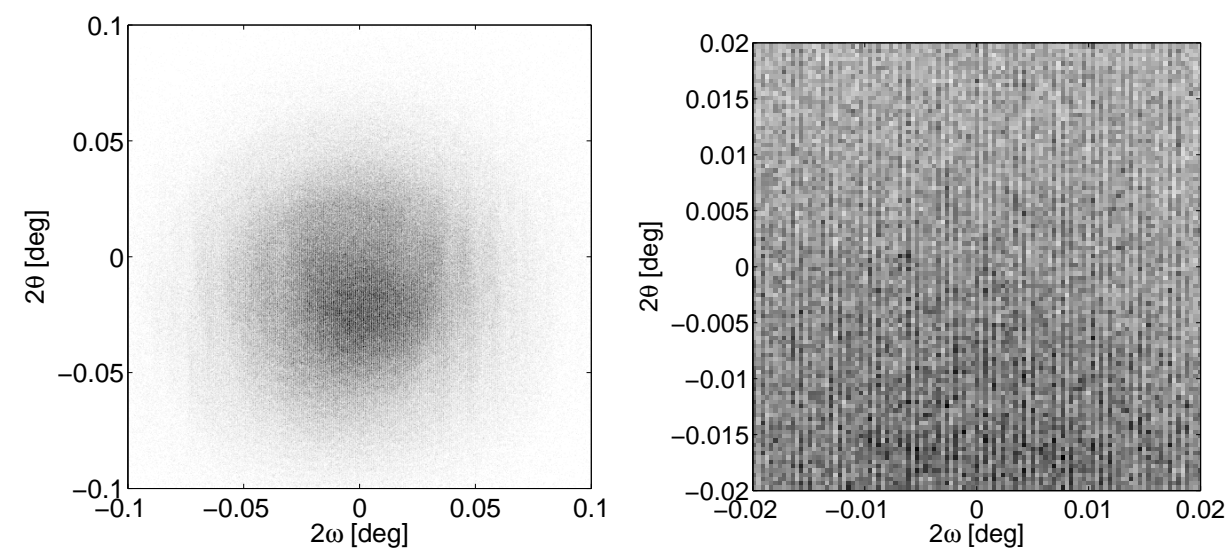

(d) single

waveguide.
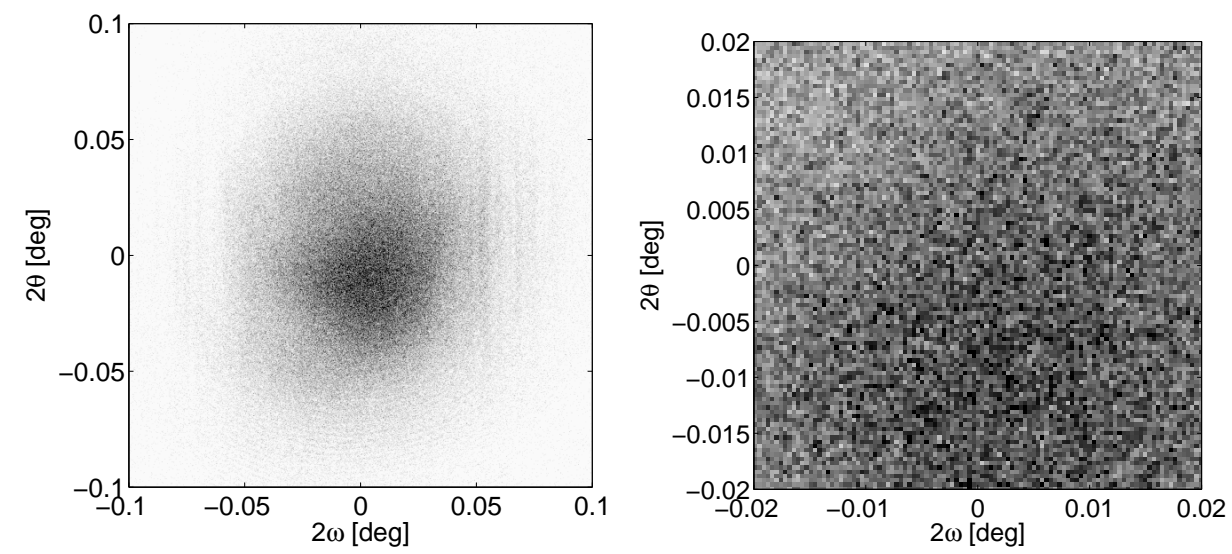

Figure 6.26: $(\mathrm{a}-\mathrm{c})$ show the intensity patterns measured behind $Y$ double waveguides with different exit distances $d_{s}$. The images on the right show enlarged sections of the centres of the patterns. (d) shows the corresponding pattern of a single waveguide for comparison. 


\subsection{Conclusions}

A variety of one-dimensionally and two-dimensionally confining waveguides was designed, fabricated and characterised. The most important parameters are the choice of materials, the cross-sectional dimensions of the guiding core, and the length of the waveguide (Section 6.1). In particular, the length of the waveguide determines the photon energy range within which a waveguide is best suited for specific applications.

Polymer/silicon waveguides were fabricated by standard thin-film and electron-beam lithography techniques (Section 6.2 ) with guiding core dimensions down to $\approx 30 \mathrm{~nm}$. The two-dimensionally confining waveguides have an approximately rectangular cross-section and the electric field inside the waveguides can be calculated using the finite-difference scheme presented in Chapter 3 .

Direct coupling was first studied using planar waveguides. It was shown that direct coupling of a pre-focused beam into a single-mode waveguide may provide a very high density of flux and small beam dimensions (Section 6.4), since the small beam dimensions provided by the waveguide are thus combined with the gain in flux density provided by the pre-focusing device. Monochromatic flux exceeding $10^{8}$ photons per second was obtained from a single-mode polyimide/silicon waveguide with a guiding layer thickness of $32 \mathrm{~nm}$ at an undulator beamline equipped with a crossed pair of Kirkpatrick-Baez mirrors for pre-focusing.

The theory of coherent propagation of guided modes was verified by an energy-resolved measurement of the far-field diffraction pattern of a planar waveguide. Therefore, a white beam was coupled into the front face of a planar waveguide supporting two guided modes and the far-field diffraction pattern was measured using an energy-dispersive detector (Section 6.5). The energy dependence of the propagation constants leads to characteristic oscillations in the energy spectrum of the far-field diffraction pattern, clearly demonstrating that the electric field at the exit of a waveguide is indeed different from that expected behind a hypothetical small slit of the same size.

The method of direct coupling with a pre-focused beam was then extended to twodimensionally confining waveguides (Section 6.6). Monochromatic flux in the order of $10^{6}-10^{7} \mathrm{ph} / \mathrm{s}$ was obtained from waveguides with guiding core dimensions of 30-100 nm. Regarding the flux requirements estimated in Section 5.3.2, this flux should be sufficient for imaging experiments with exposure times of a few seconds.

Furthermore, it was shown that arrays of two-dimensionally confining waveguides can be characterised without the need for a pre-focused undulator beam. Waveguide gratings were prepared and characterised using an unfocused beam (section 6.7). In a parallel beam, the density of flux at the exit of the waveguide may be further increased using tapered waveguides (Section 6.8). However, the angular acceptance of tapered waveguides was found to decrease significantly with an increasing gain in flux density, which makes tapered waveguides less useful for direct coupling with a pre-focusing device. 
Finally, true guiding of X-rays in the sense of changing the direction of propagation was demonstrated for curved two-dimensionally confining waveguides (Section 6.9). The waveguides were curved up to a deflection angle of $2^{\circ}$ and the transmission of curved waveguides was found to decrease strongly with an increasing curvature. However, a very slight curvature is sufficient to separate two coherently illuminated waveguides from each other. The presented Y double waveguides may thus provide two coherent waveguide beams for off-axis holography experiments without significant losses. 


\section{Experiments II: Waveguide-based imaging}

A very promising application of two-dimensionally confining $X$-ray waveguides is lensless imaging. This chapter presents proof-of-principle imaging experiments carried out in the different imaging regimes introduced in Chapter 5.

Section 7.1 introduces brilliance as the most important quantity characterising the quality of an $X$-ray beam and estimates the brilliance requirements for waveguide-based imaging experiments. The experiment setup (Section 7.2) and the preparation of appropriate test samples (Section 7.3) are then presented.

It is shown how comparably large objects may be directly recognised in a recorded image (Section 7.4), while the holographic reconstruction of smaller objects imaged in the holographic regime is demonstrated in Section 7.5. Moreover, we image micron-sized $\mathrm{Cu}$ islands to illustrate potential applications in the investigation of small clusters (Section 7.6). Finally, it is shown how holographic reconstruction may be significantly improved by using the beam of a second waveguide as a reference wave for off-axis holography (Section 7.7).

\section{$7.1 \quad$ Brilliance}

The quality of an X-ray beam depends on photon flux (photons per second), collimation of the beam (in horizontal and vertical direction, both given in milliradian), size of the source (in $\mathrm{mm}^{2}$ ), and spectral bandwidth. All these quantities are combined in the definition of brilliance* ANM01]:

$$
B r=\frac{\text { number of photons per second }}{(\mathrm{mrad})^{2}\left(\mathrm{~mm}^{2} \text { source area }\right)(0.1 \% \text { bandwidth })} .
$$

Brilliance may not be increased by optical elements like lenses or mirrors. In fact, many optical elements significantly decrease brilliance as a result of limited efficiency. Thus, by demanding a certain flux from a waveguide, one can estimate the required minimum brilliance of the primary beam.

For imaging applications, let us demand a flux of $10^{7} \mathrm{ph} / \mathrm{s}$ for illuminating $\approx 10^{5}$ pixels with exposure times in the order of secondst. Assuming a typical spectral bandwidth of $10^{-4}$, a divergence of $2 \times 2 \mathrm{mrad}^{2}$ and a guiding core cross-section of $50 \times 50 \mathrm{~nm}^{2}$,

*Note that in the literature the words "brightness" or "spectral brightness" are also used to describe this quantity $\mathrm{MHK}^{+} 05$.

${ }^{\dagger}$ Note that Poissonian noise depends on the number of detected photons, which is nearly an order of magnitude smaller than the number of photons provided by the waveguide beam [Ollon. 
this corresponds to a brilliance of $B r=10^{16} \mathrm{ph} /\left(\mathrm{s} \mathrm{mrad}^{2} \mathrm{~mm}^{2} 0.1 \%\right.$ bandwidth $)$. Assuming furthermore a transmission of $10 \%$ for the waveguide, the required brilliance of the incident beam is increased by one order of magnitude more. This high brilliance is currently only available at third generation synchrotron sources [BEW05] like undulator beam-lines at the European Synchrotron Radiation Facility in Grenoble. The imaging experiments are thus again carried out at beamline ID22, providing brilliance higher than $10^{19} \mathrm{ph} /\left(\mathrm{s} \mathrm{mrad}^{2} \mathrm{~mm}^{2} 0.1 \%\right.$ bandwidth $)$. For comparison, typical brilliance of second generation synchrotron sources is in the order $10^{14} \mathrm{ph} /\left(\mathrm{s} \mathrm{mrad}^{2} \mathrm{~mm}^{2} 0.1 \%\right.$ bandwidth $)$ and typical tube sources provide a brilliance of $10^{8} \mathrm{ph} /\left(\mathrm{s} \mathrm{mrad}^{2} \mathrm{~mm}^{2} 0.1 \%\right.$ bandwidth) [ANM01.

However, in a real experiment, brilliance of the waveguide as a source is significantly below the value one would expect only considering losses due to absorption in the waveguide. For illustration, let us consider the result of Section 6.6. The presented waveguide provides $3.5 \times 10^{6} \mathrm{ph} / \mathrm{s}$ on a cross-section of $25 \times 47 \mathrm{~nm}^{2}$ (full width at half maximum), a divergence of $\approx 2 \mathrm{mrad}$, and a spectral bandwidth of $\approx 10^{-4}$. This corresponds to $\approx 10^{16} \mathrm{ph} /\left(\mathrm{s} \mathrm{mrad}^{2} \mathrm{~mm}^{2} 0.1 \%\right.$ bandwidth $)$, which is about three orders of magnitude below brilliance of the beamline. These losses are due to

- Limited transmission of the waveguide: Transmission of the presented two-dimensionally confining waveguides is typically $5 \%$, corresponding to brilliance losses of more than one order of magnitude.

- Brilliance losses of the pre-focusing device: Although the Kirkpatrick-Baez mirrors used in the presented experiments provide a high gain in flux density and high efficiency regarding flux, efficiency regarding brilliance is much lower. During the experiments presented in Section 6.6, we measured a flux of $3.3 \times 10^{11} \mathrm{ph} / \mathrm{s}$ in a focal spot of $2.5 \times 3.8 \mu \mathrm{m}^{2}$ with a divergence of $\approx 1 \mathrm{mrad}$. This corresponds to brilliance losses of more than one order of magnitude compared to nominal brilliance of the beamline.

- Divergence mismatch of the pre-focusing device: Collimation of the pre-focused beam is better than the angular acceptance of the waveguide by a factor of around two. Taking into account collimation in two directions, this furthermore decreases brilliance by a factor of approximately four.

\subsection{Experiment setup}

Imaging experiments were carried out at the undulator beamline ID22 [STMC $\left.{ }^{+} 05\right]$ of the European Synchrotron Radiation Facility (ESRF) in Grenoble. The experiment setup is sketched in Fig. 7.1. The undulator beam is pre-focused by two perpendicularly crossed Rh-coated mirrors in the Kirkpatrick-Baez (KB) geometry [KB48, $\mathrm{HCL}^{+} 03$, $\mathrm{DRH}^{+} 02$, providing a flux of between $10^{11} \mathrm{ph} / \mathrm{s}$ and $10^{12} \mathrm{ph} / \mathrm{s}$ in a focal spot of $\approx 3.5 \mu \mathrm{m}$ in horizontal 


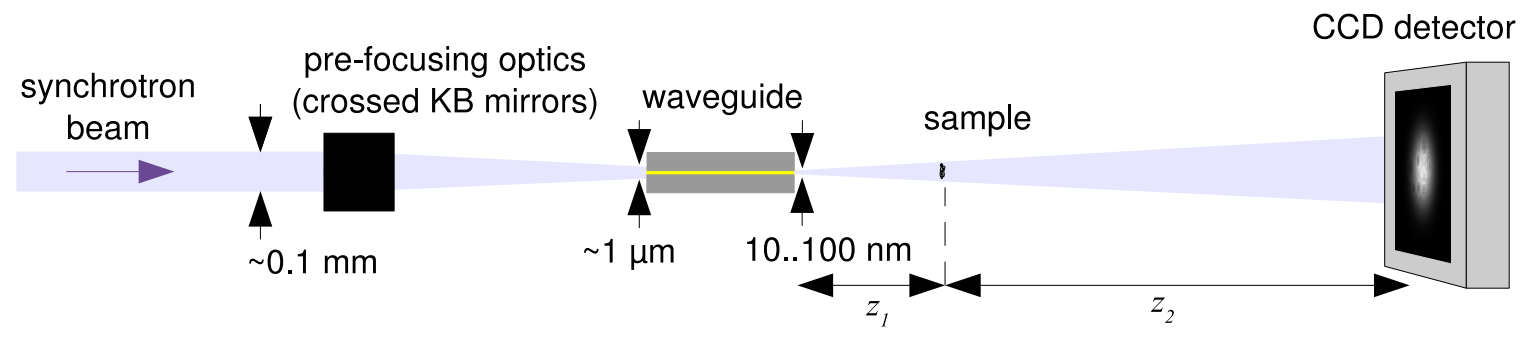

Figure 7.1: Experiment setup for waveguide-based imaging: The entrance of the waveguide is placed in the focal spot of two perpendicularly crossed Kirkpatrick-Baez mirrors. The waveguide further reduces the beam cross-section dimensions and a magnified image (or Fresnel diffraction pattern) of a sample placed in the waveguide beam is recorded with a CCD detector.

and $\approx 1 \mu \mathrm{m}$ in vertical direction (full width at half maximum). At a photon energy of $10.4 \mathrm{keV}$, a back-illuminated CCD detector with $1340 \times 1300$ pixels and a pixel size of $20 \times 20 \mu^{2}$ (Princeton Instruments PI-SX:1300) was used to obtain both high resolution and high detection efficiency Ollon. The detector was situated $3.00 \mathrm{~m}$ downstream of the waveguide and the waveguide beam typically covered a region of approximately $500 \times 500$ pixels.

Alignment of the waveguide in a focused synchrotron beam and positioning of a sample in the waveguide beam require a more elaborate instrumentation than provided at standard synchrotron beamlines. Therefore, the waveguide and the sample were positioned in the beam by means of a specifically designed instrument called the "mobile and modular waveguide stage" [Ollon. The instrument was designed and constructed by Christoph Ollinger of the Institut für Röntgenphysik. It offers all necessary translational and rotational degrees of freedom and has been installed and tested on a variety of synchrotron beamlines. Positioning of both the waveguide and the sample is carried out by stepping motors with optical encoders, combining a nominal precision of $\approx 15 \mathrm{~nm}$ with a travel range of several centimetres. The instrument thus potentially offers to combine waveguide-based lensless projection imaging and waveguide-based scanning microscopy in a single instrument.

\subsection{Preparation of test samples}

A variety of test samples was fabricated for different imaging regimes. For direct imaging, comparably large samples with dimensions well above $1 \mu \mathrm{m}$ are best suited. To demonstrate imaging of amplitude objects, tungsten wires with a diameter of $\approx 4.5 \mu \mathrm{m}$ (Luma Metall, Sweden) were used (see Fig. 7.2.

As phase objects, Au structures with a thickness of $150 \mathrm{~nm}$ were prepared using electronbeam lithography and a lift-off process (see Fig. 7.3). For this purpose, a PMMA resist layer with a thickness of $200 \mathrm{~nm}$ was spin-coated on a thin Si foil with a thickness of $\approx 150 \mathrm{~nm}$ (prepared at the Institut für Röntgenphysik [Med94, MH95]). After exposure, 


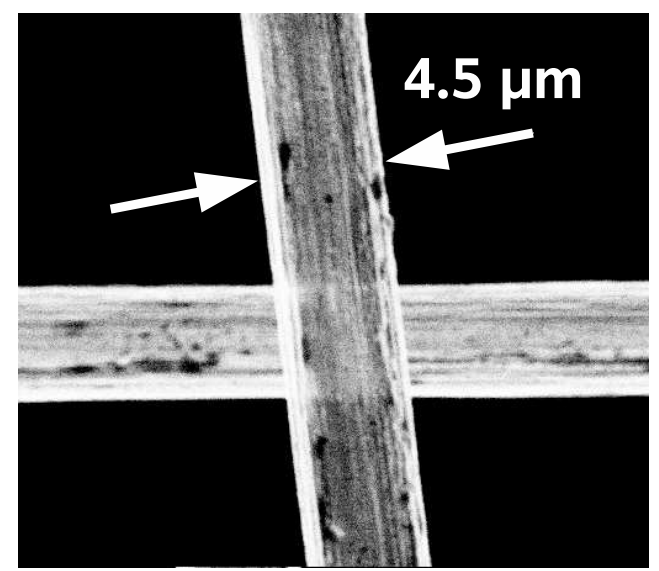

Figure 7.2: Scanning electron microscopy image of crossed tungsten wires.

the resist is developed as described in Section 6.2. Then, a thin (3-4 nm) Cr adhesive layer and the Au layer are deposited by electron-beam evaporation. The remaining parts of the PMMA layer are finally removed in a 9:1 mixture of dichloromethane and acetone (1525 minutes). For imaging in the holographic regime, Au structures with lateral dimensions down to several hundred nanometres have been prepared.

Off-axis holography was carried out with tungsten tips fabricated by standard preparation techniques in field ion microscopy $\left[\mathrm{DEH}^{+} 04\right]$. The tips were provided by the Institut für Materialphysik (University of Göttingen). The radius of curvature at the end of the tips is typically $10-20 \mathrm{~nm}$ and the diameter then increases to $100 \mu \mathrm{m}$.

\subsection{Direct imaging}

We start with comparably large objects that can easily be imaged in the direct-imaging regime (see Section 5.3.1). Fig. 7.4 shows the recorded image of two tungsten wires and some lithographically-prepared $\mathrm{Au}$ structures on a thin $\mathrm{Si}$ foil. The image was recorded at an effective defocusing distance of $40 \mathrm{~mm}$. The tungsten wires are strongly absorbant and may thus be considered as amplitude objects. In contrary, the thickness of the $\mathrm{Au}$ structures is only $150 \mathrm{~nm}$, leading to only weak absorption of $\approx 3.0 \%$ but a phase shift of $\approx 0.22$.

The sample is already recognised without any reconstruction procedures. As expected for amplitude objects, the tungsten wires are recognised as dark "shadows". The Au squares show a clear "edge enhancement" effect, which is characteristic for phase objects in this imaging regime. 


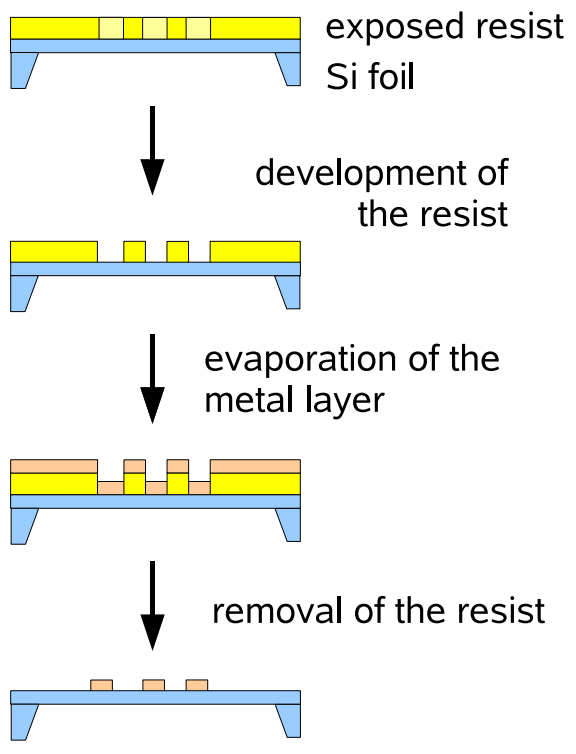

(a)

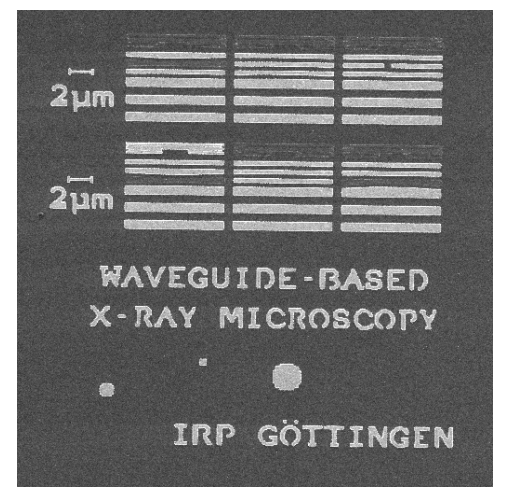

(b)

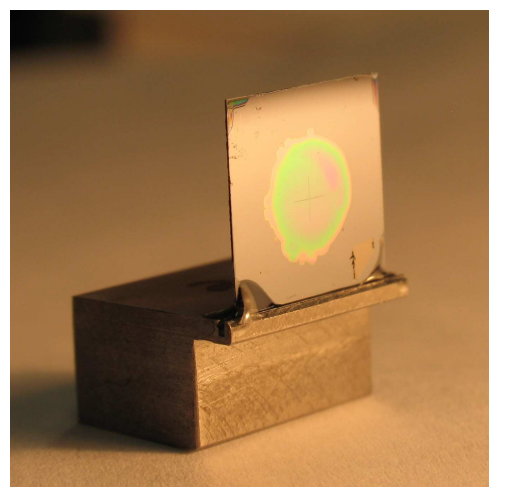

(c)

Figure 7.3: Sketch of the lift-off process for the preparation of metal test structures on a thin Si foil (a), scanning electron microscopy image of a test structure on a thin Si foil (b), photograph of a Si foil with a cross-hair structure (c).

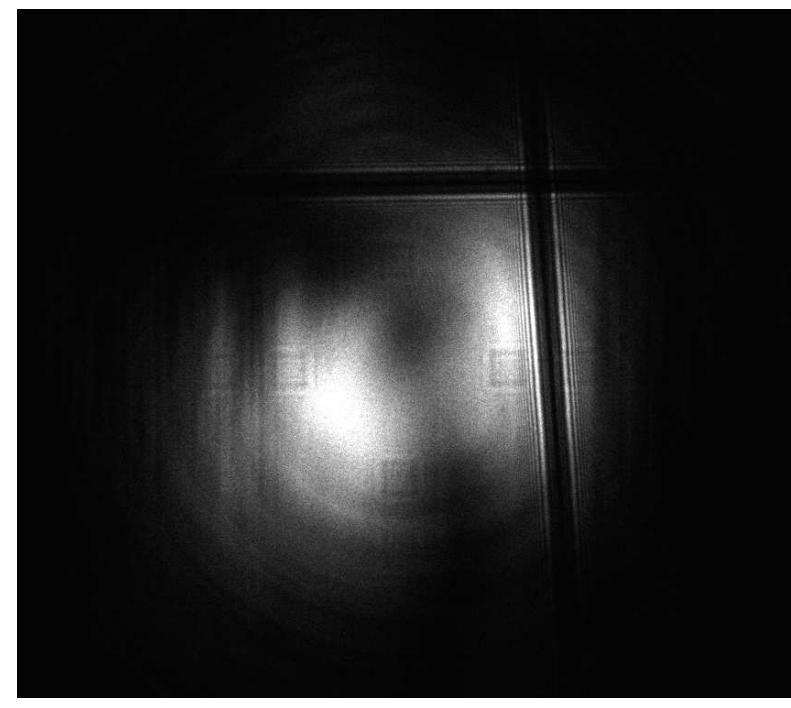

(a) Recorded image.

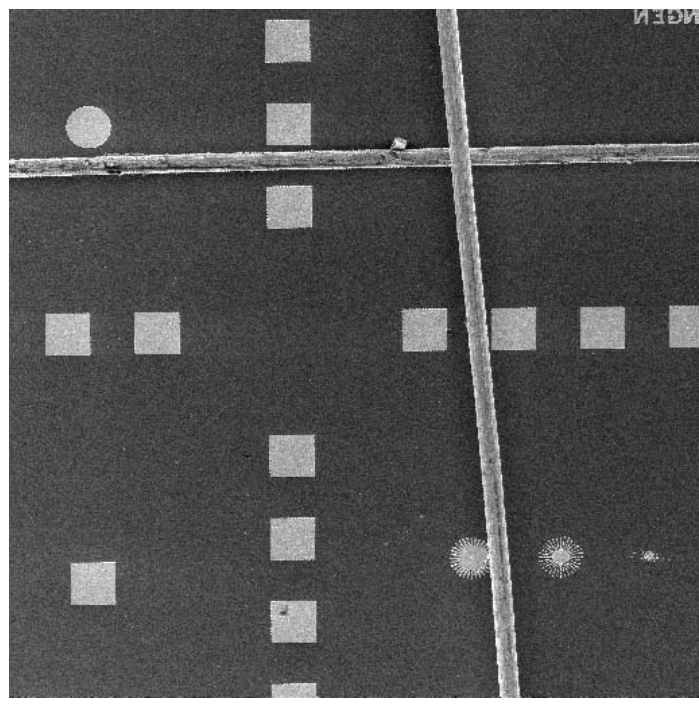

(b) Scanning electron microscopy image.

Figure 7.4: Comparably large structures may be imaged in the direct-imaging regime (a). The sample consists of lithographically-prepared Au structures with a thickness of $150 \mathrm{~nm}$ and a cross-hair of tungsten wires with a diameter of $4.5 \mu \mathrm{m}$ (b). The effective defocusing distance is $40 \mathrm{~mm}$ and the dimensions of the Au squares are $10 \times 10 \mu \mathrm{m}^{2}$. Both the wires and the squares are directly resolved in the recorded image without any reconstruction procedure. 


\subsection{Holographic imaging}

As shown in Section 5.3.1, imaging of very small objects with feature sizes close to the resolution limit requires a holographic reconstruction from the recorded image. Imaging in this regime was again investigated with lithographically-prepared Au structures on a $\mathrm{Si}$ foil. Again, the Au layer has a thickness of $150 \mathrm{~nm}$, corresponding to a phase shift of 0.22 and $3.0 \%$ absorption at $E=10.4 \mathrm{keV}$. However, we now use samples with smaller lateral dimensions than the sample imaged in the preceding section.

Fig. 7.5 shows the results obtained from a test sample imaged at an effective defocusing distance of $40 \mathrm{~mm}$. To increase the field of view, the sample is scanned in horizontal direction; the image shown in Fig. 7.5(b) is combined from 12 individual exposures. This furthermore reduces low frequency background caused by the waveguide far-field and decreases noise, thanks to better photon statistics compared to the individual holograms. Since the reconstruction process is linear, it makes no difference to the result if the measured holograms or the reconstructed complex wave fields are superposed. However, the computational time required for reconstruction increases greatly with the number of pixels. It is therefore much more favourable to reconstruct the individual holograms and superpose the reconstructed complex wave fields than to reconstruct from a larger superposition of multiple holograms recorded at different sample positions.

The comparably large bars in the upper half of the sample are again already well resolved in the recorded image. However smaller structures, namely the letters, may not be directly identified from the recorded image. Since the corresponding spatial frequencies are in the holographic regime (e.g. a spatial frequency of $\nu=(1 \mu \mathrm{m})^{-1}$ corresponds to a reduced squared frequency of $\chi \approx 15)$ imaging of these features requires a holographic reconstruction. This is shown in Figs. 7.5(c) and 7.5(d). Intensity of the reconstructed wave yields only small improvements compared to the recorded image. This is probably due to the twin-image and low absorption $(\approx 3 \%)$. The phase shift originating from the gold structure is significantly higher than absorption $(\approx 0.22)$ and the letters are indeed resolved in the phase image.

Fig. 7.6 shows similar results obtained from a test sample with half the lateral dimensions of the structure imaged in Fig. 7.5. The image was recorded at an effective defocusing distance of $6.3 \mathrm{~mm}$. To increase the field of view, again multiple individual holograms were taken while the sample was scanned through the beam. The bars in the upper half have dimensions in the micron range and the corresponding spatial frequencies $\nu \leq 10^{6} \mathrm{~m}^{-1}$ are still in the direct imaging regime (see Fig. 5.9p). Theses features are again directly resolved in the recorded image. However, the letters at the bottom have a height of $\approx 1.8 \mu \mathrm{m}$. Accordingly, spatial resolution well into the sub-micron range is necessary to resolve the text; and the corresponding spatial frequencies are in the holographic regime. The letters may not be unambiguously recognised in the recorded image (Fig. 7.6(b) and a holographic reconstruction is necessary. The phase of the reconstructed wave then allows the identifi- 


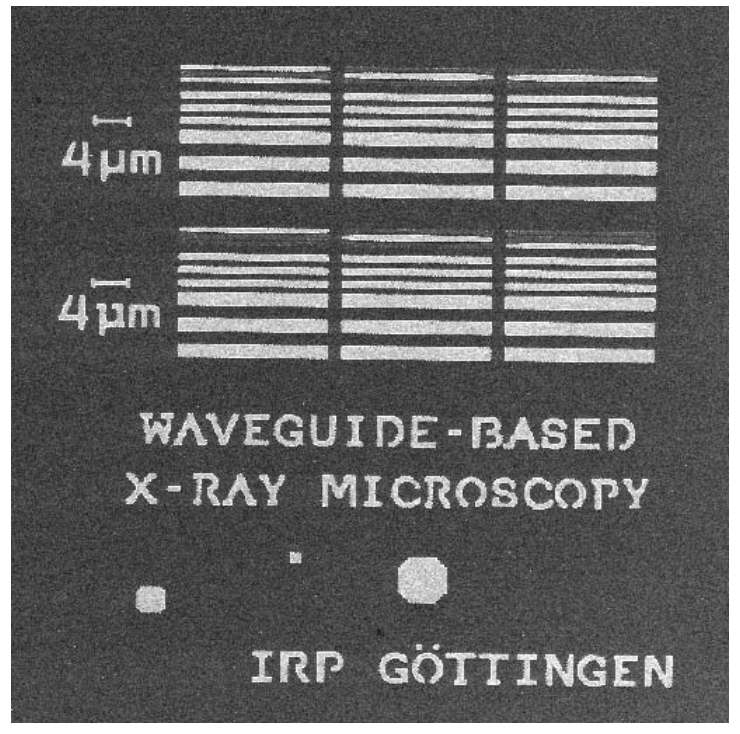

(a) Scanning electron microscopy image of the sample.

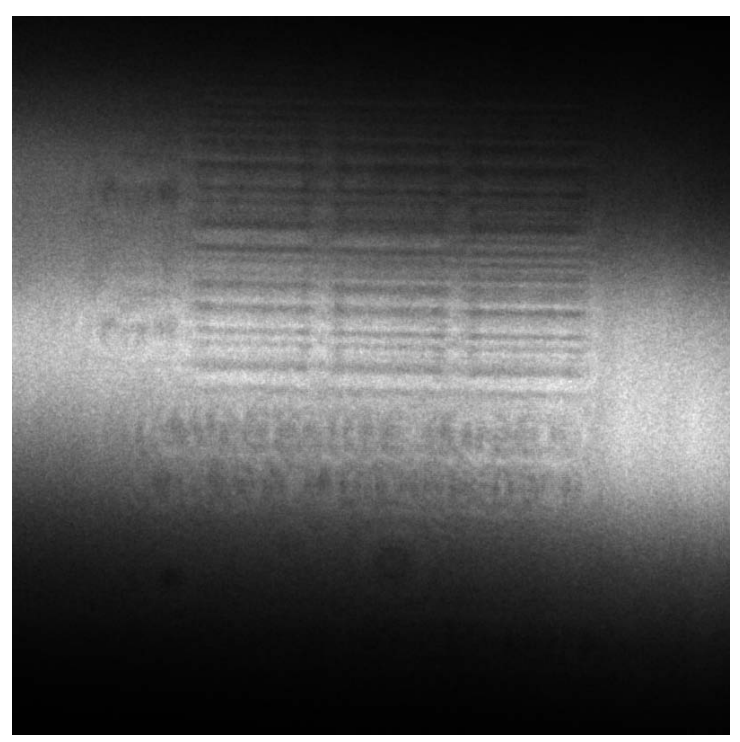

(c) Intensity of the reconstructed wave.

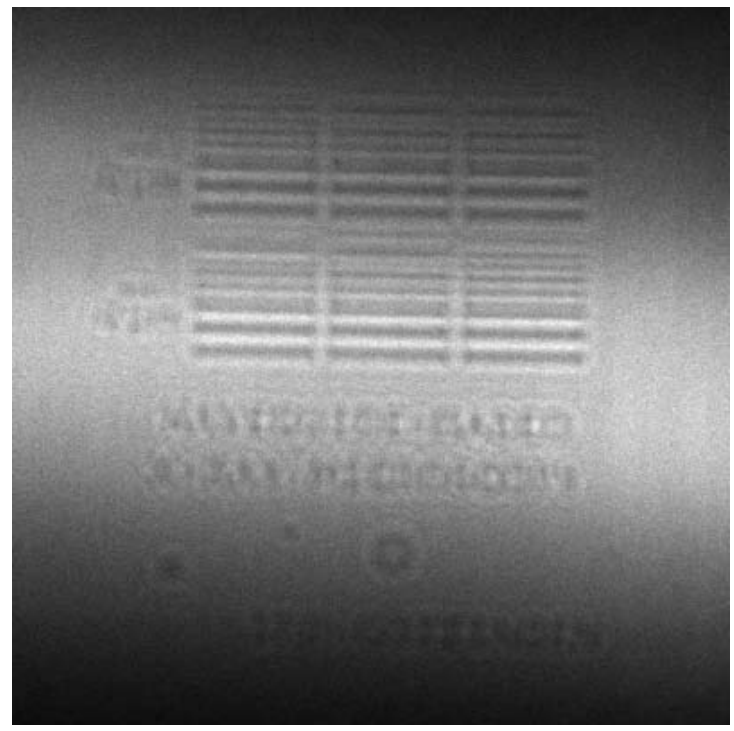

(b) Superposition of 12 in-line holograms recorded at different horizontal positions.

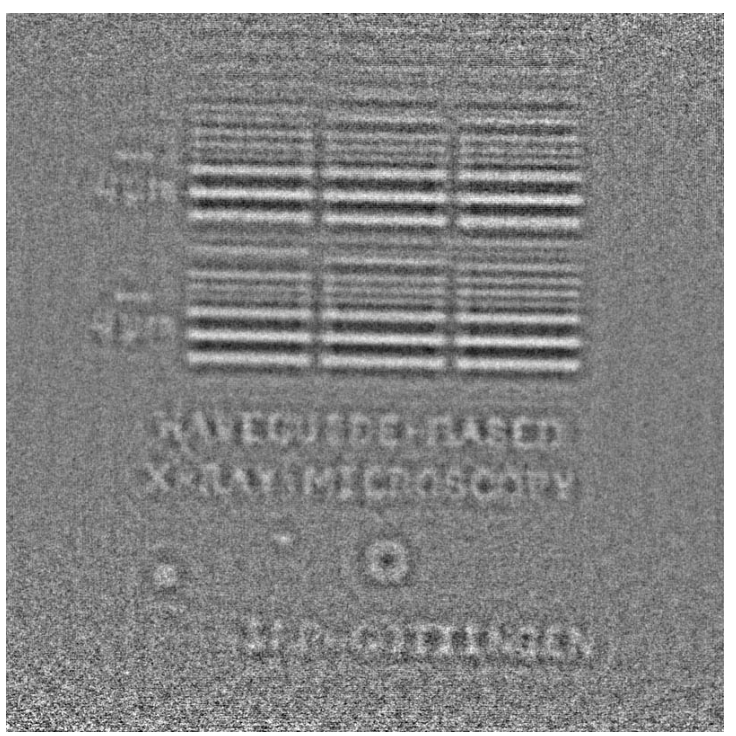

(d) Phase of the reconstructed wave (scaled between \pm 0.3$)$.

Figure 7.5: In-line holography with a lithographically-prepared test sample (a). The sample consists of Au structures with a thickness of $150 \mathrm{~nm}$ prepared on thin Si foil. The comparably large bars are directly recognised in the hologram (b). Intensity of the reconstructed wave (c) yields only small improvements compared to the recorded hologram. However, the letters are finally resolved in the phase of the reconstructed wave (d). The photon energy is $10.4 \mathrm{keV}$ and the effective defocusing distance is $40 \mathrm{~mm}$. 


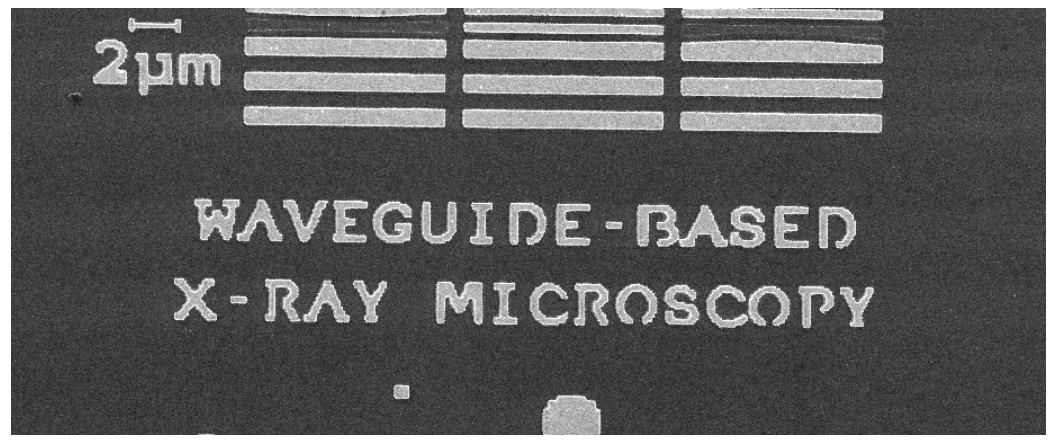

(a) Scanning electron microscopy image of the sample.

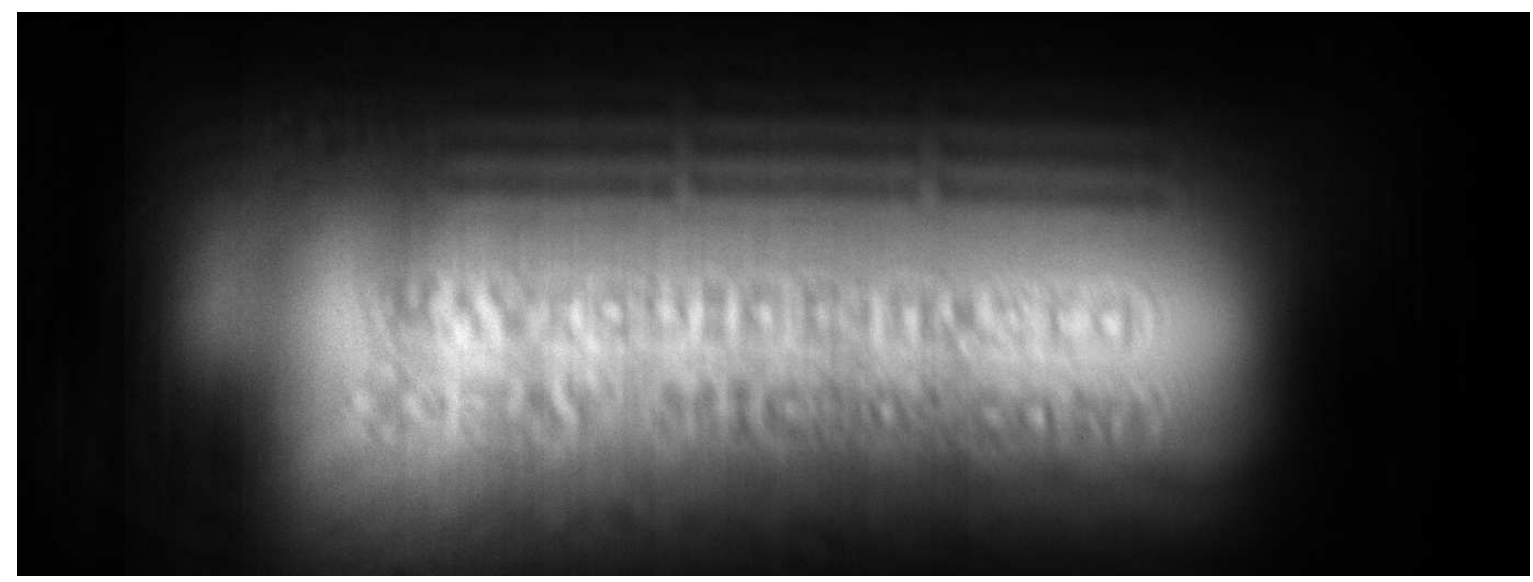

(b) In-line hologram (superposition of 11 holograms recorded at different positions shifted by $4 \mu \mathrm{m}$ ).

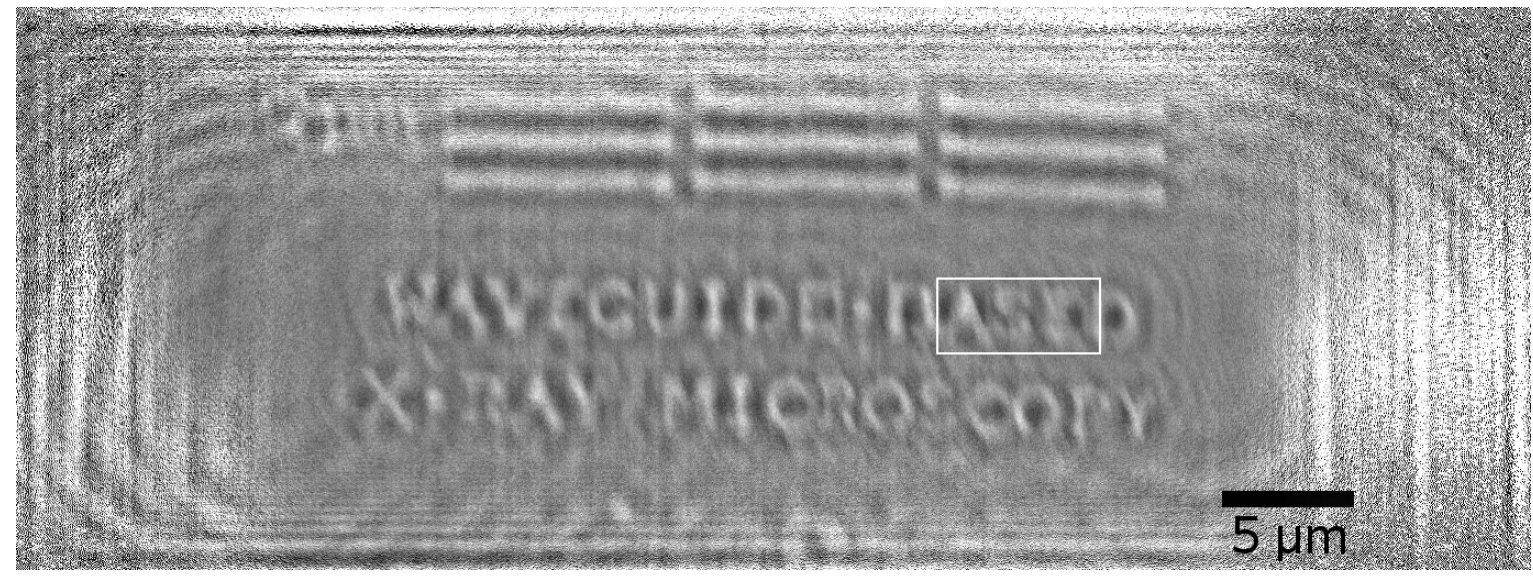

(c) Reconstructed phase (scaled between \pm 0.3 ). The box indicates the section enlarged in Fig. 7.7

Figure 7.6: In-line holography with a lithographically-prepared test sample (a). The sample consists of Au structures with a thickness of $150 \mathrm{~nm}$ prepared on thin Si foil. Larger structures are already recognised in the recorded hologram (b) and the letters are clearly identified in the phase of the reconstructed wave (c). The photon energy is $10.4 \mathrm{keV}$ and the effective defocusing distance is $6.3 \mathrm{~mm}$. 


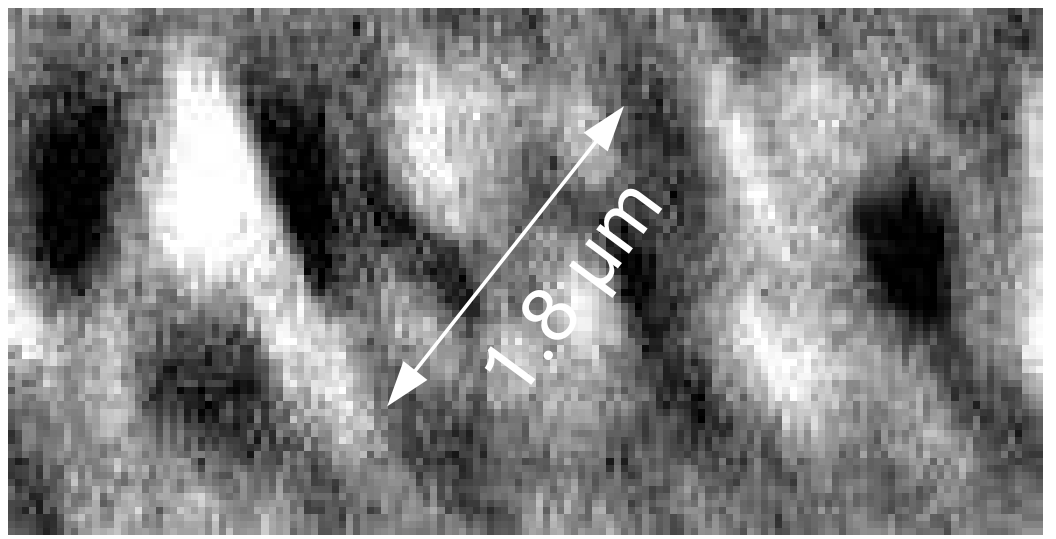

Figure 7.7: Enlarged view of the box indicated in Fig. 7.6. The arrow indicates a resolution of $360 \mathrm{~nm}$ in the respective direction. However, the horizontal bar in the middle of the letter " $E$ " is not well resolved and the right edge of the letter " $A$ " appears sharper than the left one. Accordingly, spatial resolution seems to be lower in other directions. (Contrast is optimised for clarity.)

cation of the letters (Fig. 7.6(c) ). From the enlarged section shown in Fig. 7.7, one may deduce a spatial resolution of $\approx 360 \mathrm{~nm}$, since five dark or bright regions may be identified along the arrow with a length of $1.8 \mu \mathrm{m}$. Note that the horizontal bar in the middle of the letter "E" is not well resolved and the right edge of the letter "A" appears sharper than the left one. The spatial resolution therefore seems to be lower in other directions.

Although the holographically reconstructed images allow the identification of structures with feature sizes well in the sub-micron range, the images also show clearly visible artifacts. We find wavy structures around the reconstructed text and dark regions around the letters corresponding to a phase shift in opposite direction. These artifacts are related to the twin-image, which is a fundamental problem of in-line holography. However, the twinimage problem may be overcome by off-axis holography, which is considered in Section 7.7 .

\subsection{Imaging of $\mathrm{Cu}$ islands}

To illustrate possible future applications in the studies of small "clusters" or "nanodots", micron-sized $\mathrm{Cu}$ islands prepared on a thin Si foil were studied. Although these islands are relatively large, the experiment gives a sketch of experiments that may shortly resolve smaller structures using waveguides with smaller guiding core dimensions and higher flux. In the next step, one may for instance consider in-situ studies of related growth phenomena or studies of embedded particles.

The $\mathrm{Cu}$ islands were prepared at the Institut für Materialphysik (University of Göttingen) by pulsed laser deposition [CH94, $\left.\mathrm{KWF}^{+} 03\right]$. At a substrate temperature of approximately $220{ }^{\circ} \mathrm{C}$, an exceptionally high surface mobility of $\mathrm{Cu}$ atoms on Si results in the growth of remarkably large islands. 


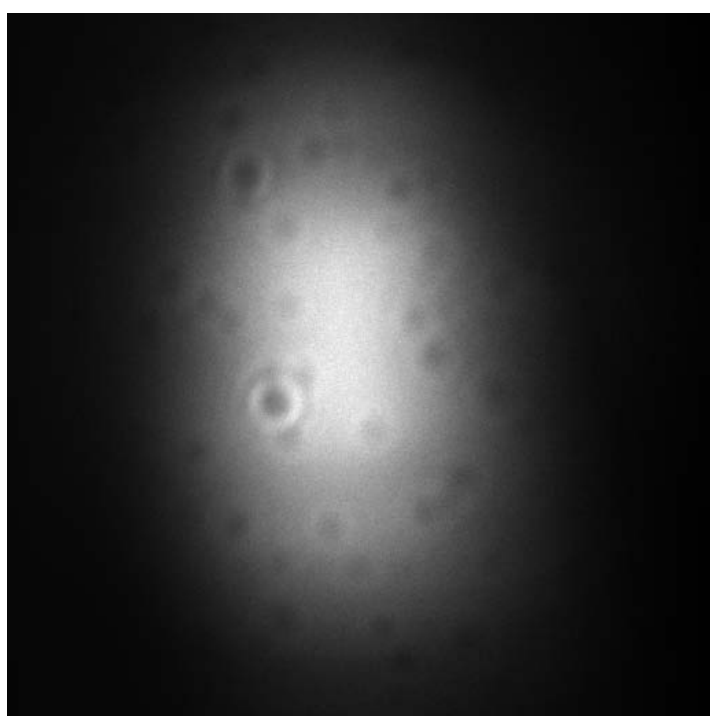

(a) Recorded image (superposition of 21 individual exposures).

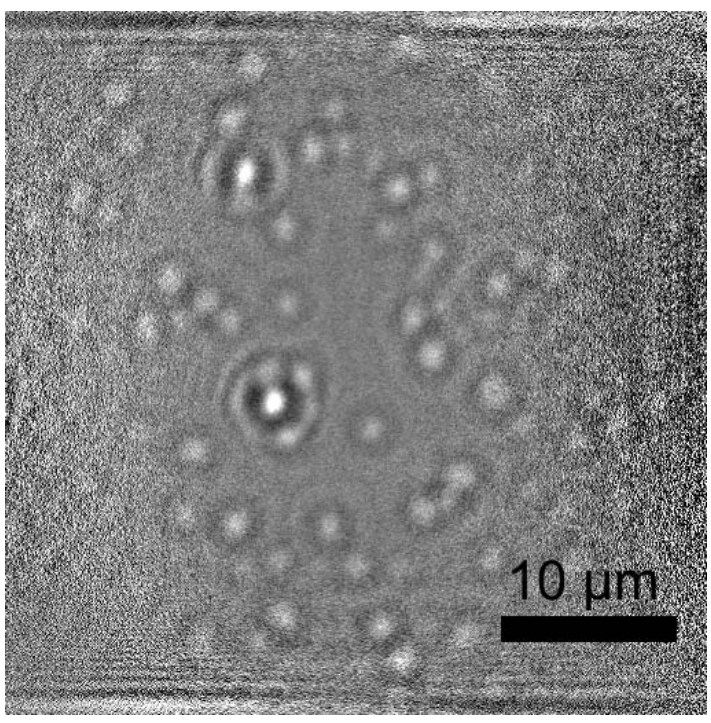

(b) Phase of the reconstructed wave.

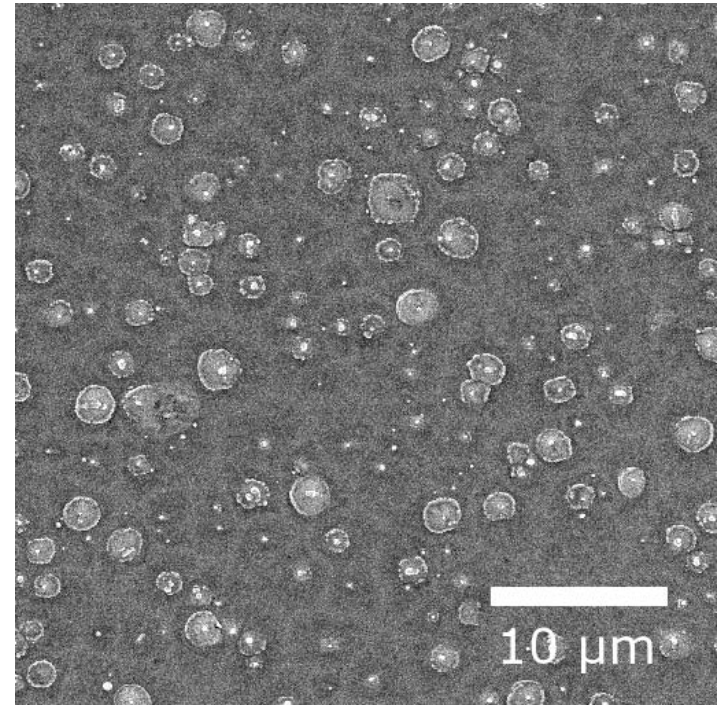

(c) Representative scanning electron microscopy image. Note the bright spots in the centres of the islands indicating the pyramidal shape.

Figure 7.8: The micron-sized pyramidal $\mathrm{Cu}$ islands are easily recognisable in the recorded image (a). The phase of the reconstruction yields an improved image and some more islands are recognised in regions where intensity is low (b). However, the image also shows artifacts owing to the twin image, for instance dark regions and ring-like structures around the two brightest spots. A representative scanning electron microscopy image (c) illustrates size and distribution of the islands. To avoid charging, the sample was coated with 10-15 $\mathrm{nm}$ AuPd for scanning electron microscopy. 
Fig. 7.8(a) shows an image recorded at an effective defocusing distance of $11.5 \mathrm{~mm}$ and photon energy of $10.4 \mathrm{keV}$. The individual islands are directly recognised in the recorded image. Again, the phase of the reconstructed wave (Fig. 7.8(b) then resolves the sample in more detail. We also find artifacts related to twin-image, in particular some ring-like structures probably around very large islands.

With the appropriate instrumentation, the sample could easily be moved very close to the waveguide until only one of the islands is illuminated. This would enable the characterisation of individual islands, for instance by X-ray diffraction or fluorescence.

\subsection{Off-axis holography}

Off-axis holography experiments were carried out using a Y double waveguide with an exit distance of $d_{s}=5 \mu \mathrm{m}$ in horizontal direction (see Section 6.9.3). The tip was aligned in a vertical direction to allow scanning without disturbing the reference beam. Photon energy was $10.4 \mathrm{keV}$ and the effective defocusing distance was $1.25 \mathrm{~mm}$.

Fig. 7.9(a) shows a typical off-axis hologram recorded with a tungsten tip. The tip is already recognised as a "shadow" in the recorded hologram. There are also some halfcircular structures visible in the hologram that are not related to the sample. As shown in Fig. 7.9(b), these structures are also visible without the sample. Since these artifacts are not visible in a far-field diffraction pattern measured earlier (see Section 6.9.3, Fig. 6.26(b)), they are probably a result of beam damage of the waveguides. Phase and intensity of the reconstructed first-order wave are shown in Fig 7.9(c) and Fig 7.9(d), respectively. Although the intensity image is very noisy and the tip is not recognisable, it can be clearly seen in the phase image. The phase of the reconstructed wave exhibits very few artifacts or noise and yields a clear and unambiguous image of the sample.

Although the field of view is limited by $d_{s}$ in horizontal direction, it may be increased in vertical direction by scanning the waveguide beam over the tip. Multiple individual reconstructions may then be combined. Fig. 7.10 shows phase images of the tip combined from three individual reconstructions. Thus, a larger section of the tip becomes visible and the shape of the tip becomes recognisable in agreement with the scanning electron microscopy image shown in Fig. 7.10(c).

As evident from symmetry, each of the two individual waveguides may be used to illuminate the sample while the other one then provides the reference beam. Corresponding reconstructions are shown in Fig. 7.10(a) and Fig. 7.10(b), As expected, both results are of practically the same quality. Note that in the reconstruction the focused image moves from one first-order beam to the other when the sample is moved into the other beam.

Since the zeroth-order beam in the reconstruction of an off-axis hologram yields the same image that would be obtained from an in-line hologram, we may compare these techniques by comparing the reconstructed first-order image to the zeroth-order image. This comparison is shown in Fig. 7.11 to highlight the advantages of off-axis holography. Evi- 


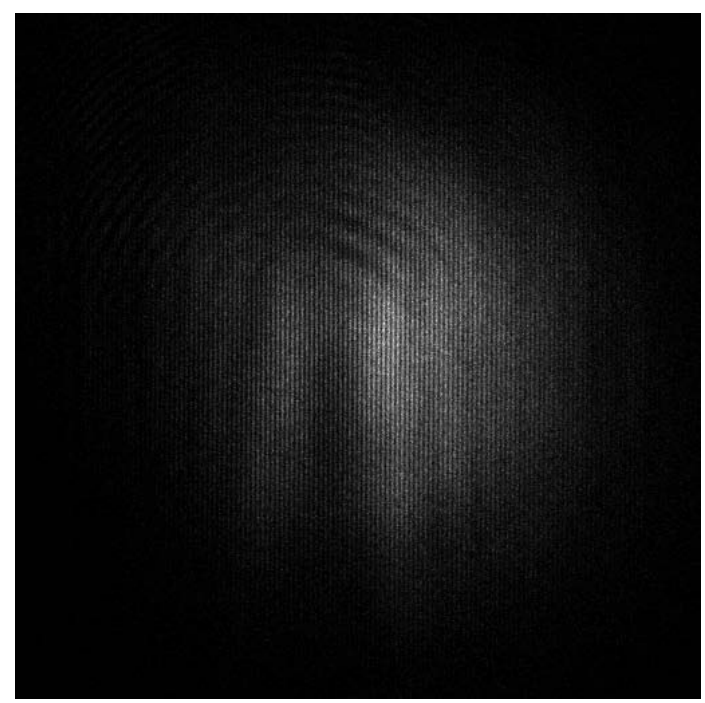

(a) Off-axis hologram of the tungsten tip.

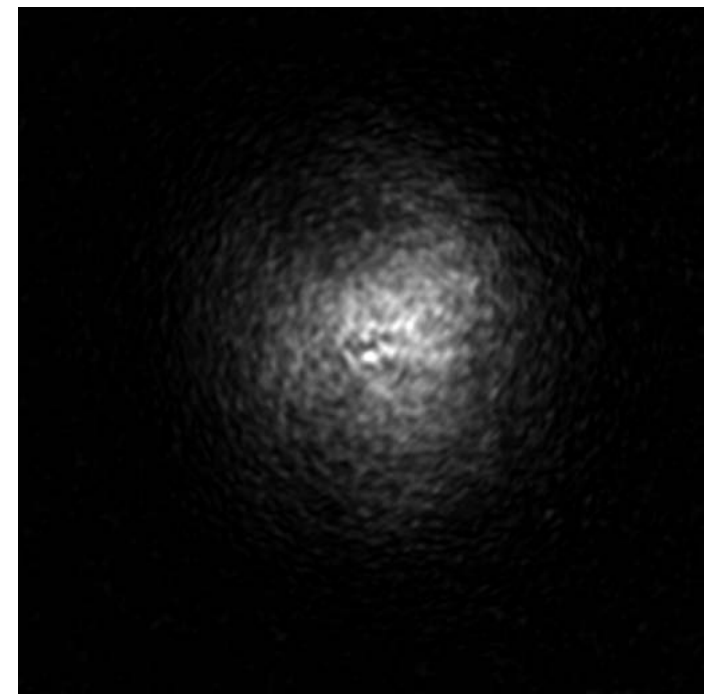

(c) Intensity of the reconstructed first-oder beam.

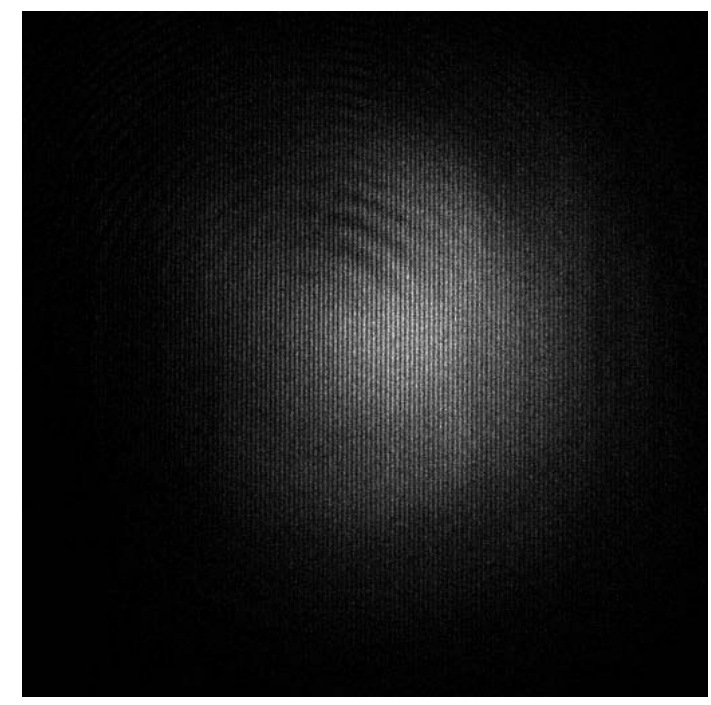

(b) Waveguide far-field without the tip.

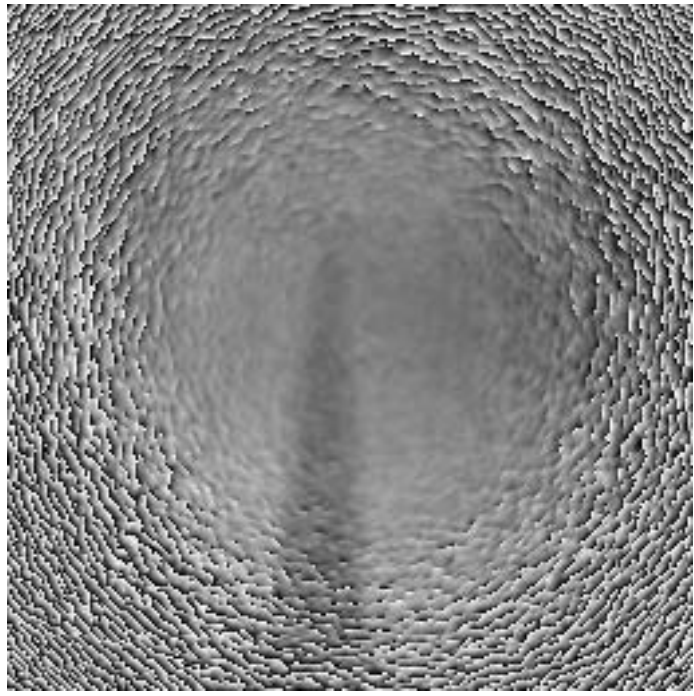

(d) Phase of the reconstructed first-oder beam.

Figure 7.9: Imaging of a tungsten tip by means of $Y$-double-waveguide-based off-axis holography $\left(d_{s}=5 \mu \mathrm{m}\right)$. The tip is already indicated by a "shadow" in the recorded hologram (a) and is clearly visible in the reconstructed phase image (d). The intensity image obtained from the reconstructed first-order is too noisy to allow an identification of the tip (c). This is probably a result of beam damage of the $Y$ double waveguide, indicated by half-circular structures in the hologram also observed in the waveguide far-field without the sample (b). Effective defocusing distance was $1.25 \mathrm{~mm}$; photon energy was $10.4 \mathrm{keV}$; exposure time was $10 \mathrm{~s}$. 


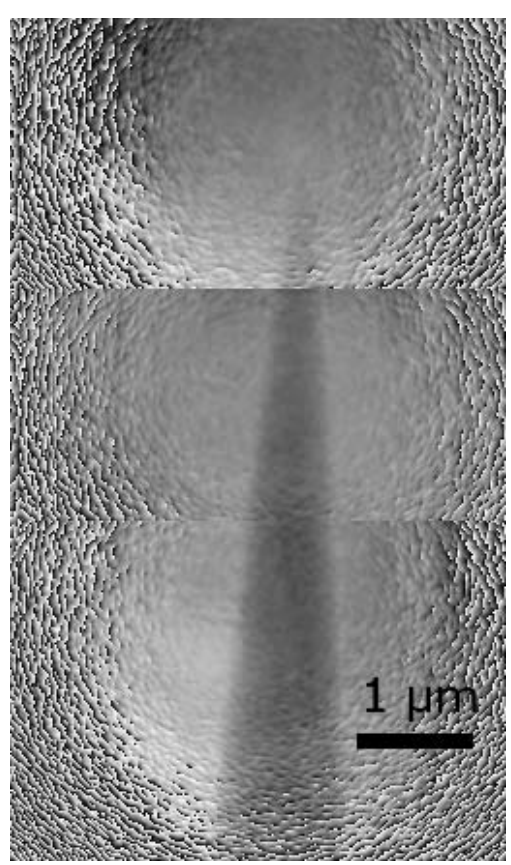

(a) Phase of the reconstructed first-order beam.

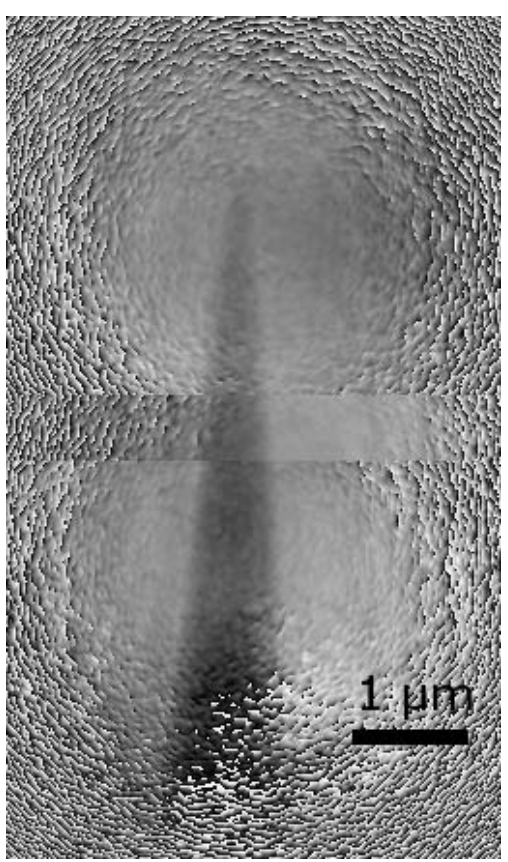

(b) Same as (a), but the tip is here illuminated by the other waveguide.

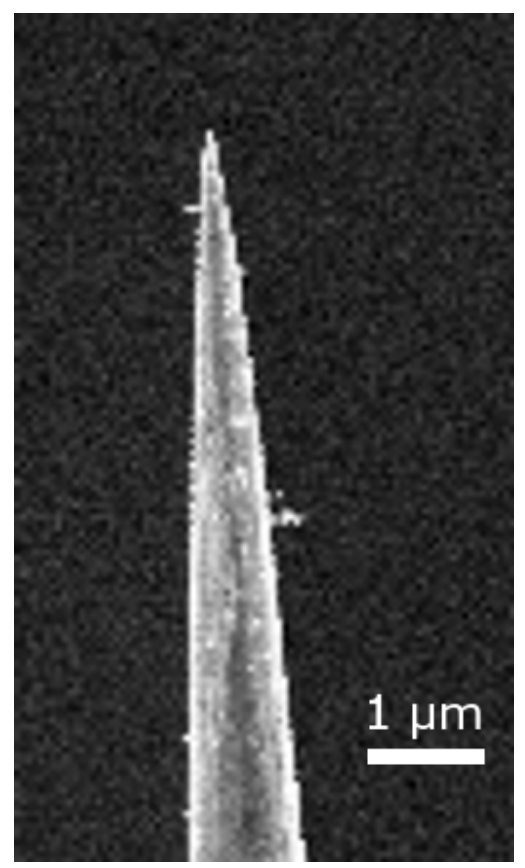

(c) Scanning electron microscopy image.

Figure 7.10: Reconstructed off-axis hologram and scanning electron microscopy image of a tungsten tip. The phase images are stitched from three individual off-axis holograms. Between (a) and (b) the tip was moved by $5 \mu \mathrm{m}$, changing the reference beam and the beam illuminating the tip. Effective defocusing distance was $1.25 \mathrm{~mm}$; photon energy was $10.4 \mathrm{keV}$; exposure time of the individual holograms was $10 \mathrm{~s}$. 


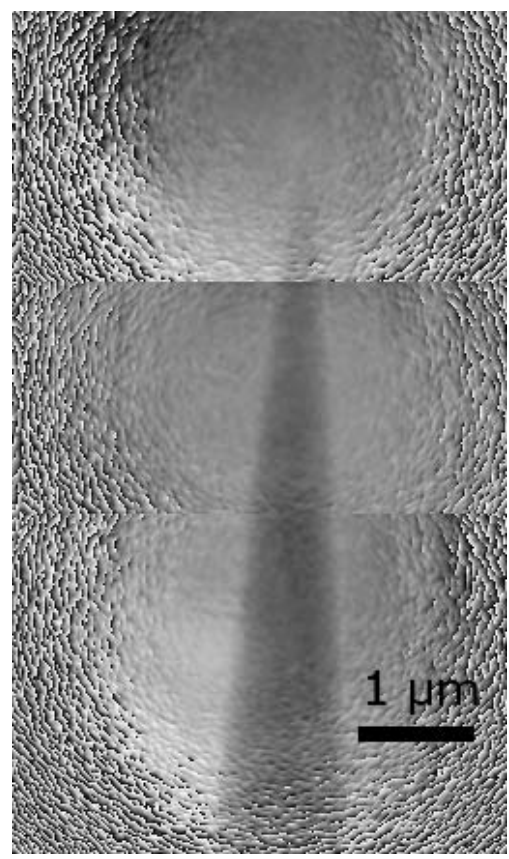

(a) Reconstructed first-order beam (off-axis reconstruction).

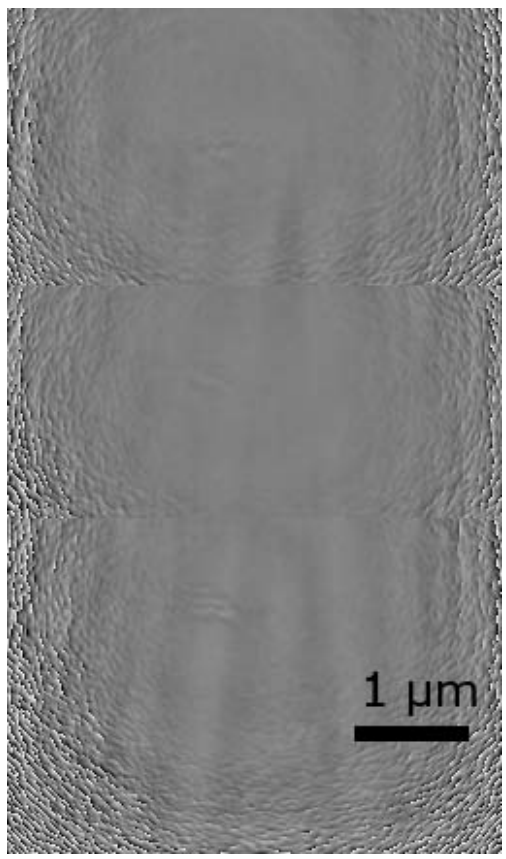

(b) Reconstructed zeroth-order beam (in-line reconstruction).

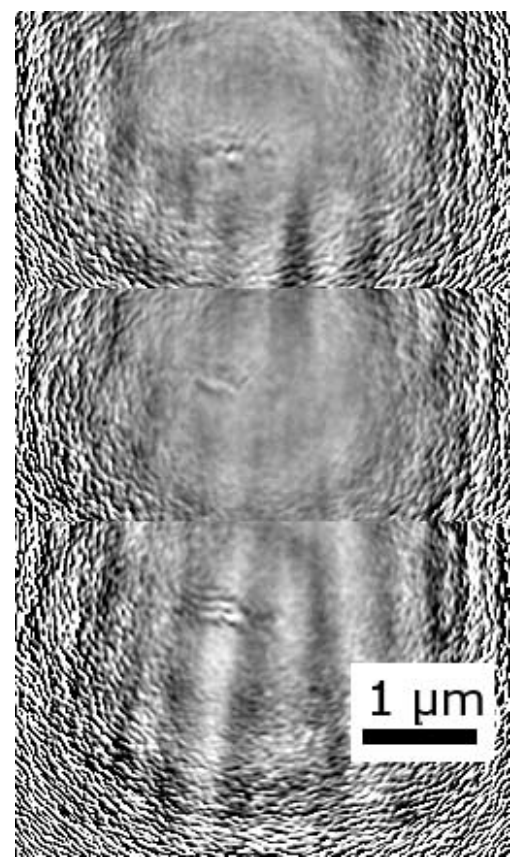

(c) Contrast-enhanced zerothorder reconstruction.

Figure 7.11: Phase images obtained from first-order (a) and zeroth-order (b, $c$ ) beams reconstructed from the same off-axis holograms. Image quality obtained from the first-order beam in off-axis holography is significantly improved compared to quality of the zeroth-order image, which corresponds to reconstruction from an in-line hologram. The images are stitched from three individual holograms to increase the field of view in vertical direction. Effective defocusing distance was $1.25 \mathrm{~mm}$; photon energy was $10.4 \mathrm{keV}$; exposure time of the individual holograms was $10 \mathrm{~s}$.

dently, the zeroth-order image shows significantly lower contrast and disturbing artifacts, i. e. a clearly visible twin-image. The first-order image exhibits much better contrast and no twin-image is observed.

Finally, we verify that the phase obtained from the reconstructed first-order beam indeed yields the phase shift originating from the sample. A homogeneous sample of thickness $t$ shifts phase of an X-ray beam by $t k \delta$. For tungsten at a photon energy $10.4 \mathrm{keV}$ we find $\delta=2.59 \times 10^{-5}$ [HGD93]. Assuming a circular cross-section with radius $R$, the corresponding phase shift of a tip located at $x=0$ is then given by

$$
\Delta \phi(x)= \begin{cases}2 \sqrt{R^{2}-x^{2}} \delta k, & \text { for }|x|<R \\ 0, & \text { else }\end{cases}
$$

Fig. 7.12 shows the calculated phase shift in comparison to the phase shift obtained from slices through the reconstructed phase image. The curves are in good agreement, indicating that the reconstructed image indeed yields the phase shift originating from the sample, i. e. enables a quantitative determination of the phase of the optical transmission function. 

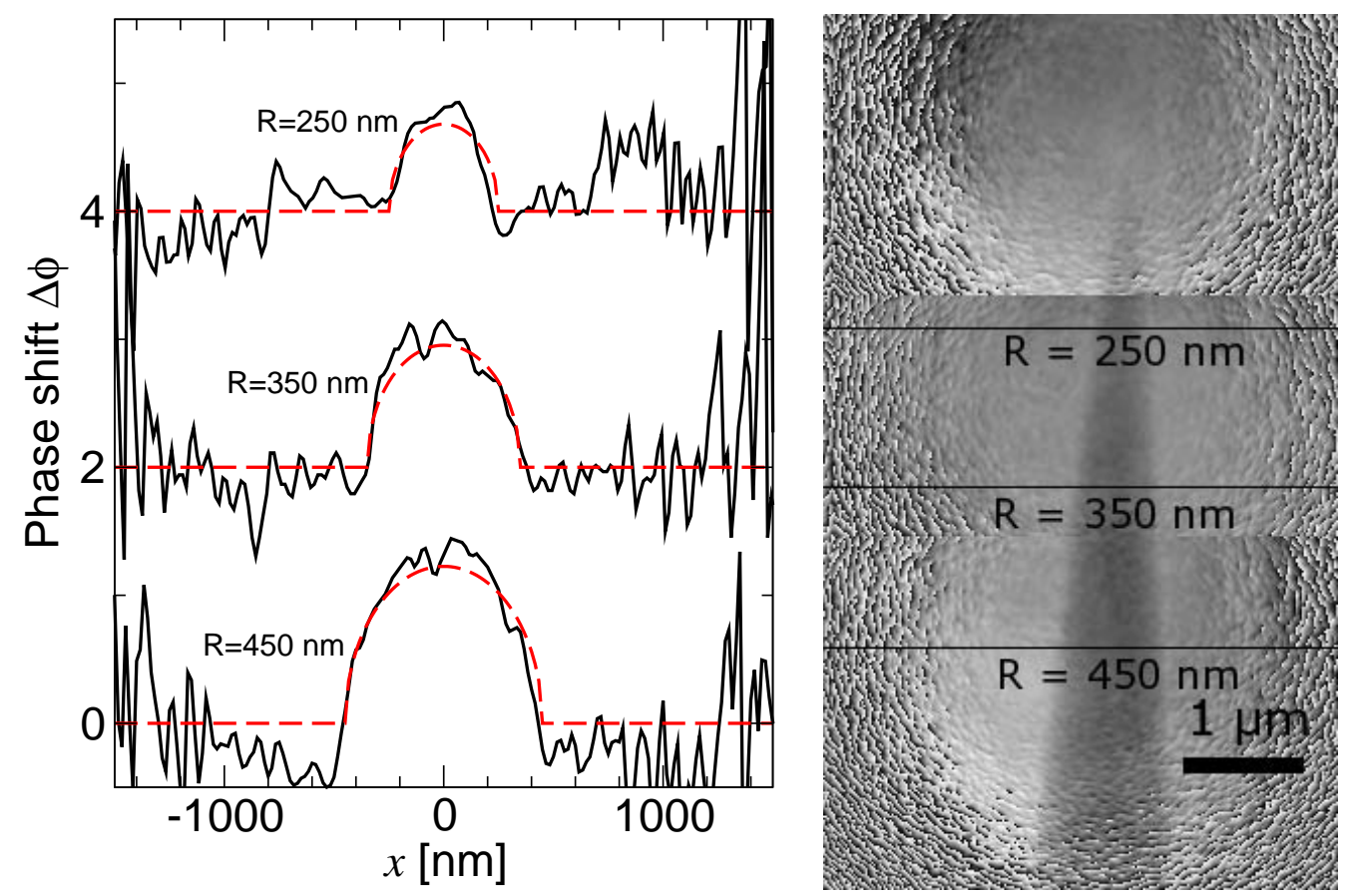

Figure 7.12: The phase shift of a tungsten tip with circular cross-section was calculated for different radii $R$ (dashed red curves) and compared to corresponding slices (black solid curves) through the phase image obtained from the reference beam hologram (right). The curves are shifted for clarity and a linear background was subtracted from the black curves.

Note the sharp edges in the reconstructed phase image, clearly seen in slices shown in Fig. 7.12. Although spatial resolution cannot directly be determined by resolving correspondingly small structures, sharpness of these edges indicates a spatial resolution in the order of $100 \mathrm{~nm}$. This roughly agrees with the nominal cross-sectional dimensions of the waveguide, which were $\approx 60 \times 60 \mathrm{~nm}^{2}$.

\subsection{Conclusions}

Proof-of-principle experiments have been carried out demonstrating the feasibility of waveguide-based lensless projection imaging. It was shown that the experiments require highly brilliant sources (Section 7.1) currently only available at third generation synchrotron facilities. Correspondingly, the experiments have been carried out at an undulator beamline of the European Synchrotron Radiation Facility in Grenoble. The experiment setup was described in Section 7.2 and appropriate test samples were prepared for the different imaging regimes (Section 7.3).

Depending on the feature size and sample-to-waveguide distance, imaging in the direct contrast regime (Section 7.4) and in the holographic regime (Section 7.5) were demonstrated. A spatial resolution of $\approx 360 \mathrm{~nm}$ was achieved by holographic reconstruction from in-line holograms. In Section 7.6, micron-sized $\mathrm{Cu}$ islands were imaged to illustrate poten- 
tial applications in the investigation of small clusters or nanodots. However, reconstruction from an in-line hologram was significantly spoiled by an inseparable twin-image.

Like in holography with visible light, the twin-image problem can be overcome by means of off-axis holography (Section 7.7). Therefore, the Y double waveguides introduced in Section 6.9.3 were applied and high-quality twin-image-free phase images were reconstructed. The method corresponds to two-beam Gabor microscopy. Although spatial resolution could not directly be determined by resolving correspondingly small structures, sharpness of the edges in the reconstructed phase image indicates a spatial resolution of $\approx 100 \mathrm{~nm}$. 


\section{Summary}

The aim of this thesis was (i) to contribute to the understanding of wave propagation in $\mathrm{X}$-ray waveguides, (ii) to design, fabricate and characterise in particular two-dimensionally confining X-ray waveguides providing beams well-suited for microscopy applications, (iii) to develop a theoretical background for waveguide-based lensless full-field imaging, and (iv) to experimentally verify the feasibility of this technique.

In Chapter 2, an introduction to the propagation of X-rays in waveguides was given and well-known analytical solutions for slab waveguides and cylindrical waveguides were presented. Since absorption is an important issue, the existing theories have been extended by the concept of "effective linear attenuation coefficients" accounting for attenuation of individual guided modes. Motivated by the lack of exact analytical solutions for many waveguide structures of practical relevance, the parabolic wave equation has been presented as a basis for numerical approximations. Furthermore, dimensionless coordinates have been introduced, enabling more general conclusions from individual solutions.

In Chapter 3, finite-difference algorithms were implemented to solve the parabolic wave equation for one-dimensionally and two-dimensionally confining waveguides. The results have been verified by comparison to analytical solutions, in particular to the extended theory for slab waveguides and cylindrical waveguides. Moreover, the algorithms allow the study of waveguide structures where exact analytical solutions are not available. This enables the easy design of X-ray waveguides for specific applications. Evidently, the algorithms can be applied to study other X-ray optical devices as well.

For many applications, the main purpose of an X-ray waveguide is to reduce beam dimensions. The same effect could in principle also be achieved with a hypothetical small slit or pinhole of the same size. However, in Chapter 4 it was shown that an aperture made of any real material has to be considered a waveguide when the diameter is far in the sub-micron range. This results from the large aspect ratio required to ensure sufficient absorption in the cladding. A critical width below which waveguiding effects have to be taken into account was quantified by analytical theory. The corresponding changes in the field inside and directly behind the waveguide were studied by finite-difference calculations. It was observed that transmission of X-ray waveguides decreases critically when the guiding core cross-section dimensions become very small and the waveguide supports only few guided modes. In particular, transmission of single-mode waveguides providing smallest beam dimensions was studied by analytical theory. Transmission of these devices depends on the required absorption of over-illuminated radiation impinging on the cladding and may be significantly below 1 . However, transmission of single-mode waveguides can be 
drastically enhanced by introducing an appropriate interlayer between the guiding core and the cladding.

In Chapter 5, lensless waveguide-based projection imaging was described by scalar wave theory. The waveguide is considered a coherent quasi-point-like source, allowing the recording of a magnified image of a sample. The finite beam dimensions at the end face of the waveguide limit the numerical aperture and thus spatial resolution. As a rule of thumb, one may expect spatial resolution to be given by the cross-sectional dimensions of the guiding core. Magnification is easily adapted by placing the sample at an appropriate distance from the waveguide. Spatial resolution therefore becomes practically independent of the detector pixel size.

Depending on the effective defocusing distance and the spatial frequencies to be resolved, different imaging regimes have been pointed out: Large objects may be imaged with "direct" contrast, i. e. absorbing parts of the sample appear as "shadows" and phase objects become visible by strongly pronounced edges. Smaller features corresponding to higher spatial frequencies close to the resolution limit can only be imaged in the holographic regime, where a diffraction pattern (or "hologram") without direct resemblance to the investigated features is recorded.

In the holographic regime, an image of the investigated features is obtained by holographic reconstruction. The experiment setup corresponds to a Gabor microscope, where a magnified in-line hologram of a sample illuminated by a point-like source is recorded. However, it is well-known that reconstruction from in-line holograms is significantly disturbed by artifacts, namely by an inseparable defocused twin image. But in particular samples with sharp edges are nevertheless clearly identified. Much better reconstructions are obtained from off-axis holography. To this end, an additional reference wave is superposed when the hologram is recorded. In the reconstruction, the twin image is spatially separated from the reconstructed sample wave and image quality is drastically enhanced compared to the results of in-line holography. A two-beam Gabor microscope applies two divergent beams for sample illumination and as a reference. Thus, a magnified off-axis hologram is recorded.

One-dimensionally and two-dimensionally confining X-ray waveguides were designed and fabricated (Chapter 6). The presented waveguides consist of a polymer guiding core in silicon and were prepared using standard thin-film technology. Two-dimensionally confining waveguides were prepared by means of electron-beam lithography. The fabricated waveguides were then characterised experimentally. The presented experiments (i) verify the given analytical theory of wave propagation in one-dimensionally and two-dimensionally confining X-ray waveguides, (ii) are in agreement with the finite-difference calculations, (iii) yield sufficient flux for imaging experiments with two-dimensionally confining waveguides, and (iv) demonstrate production of two coherent waveguide beams for off-axis holography using Y double waveguides. In particular, monochromatic beams with cross-section dimen- 
sions down to $25 \times 47 \mathrm{~nm}^{2}$ and flux of typically $10^{6}-10^{7} \mathrm{ph} / \mathrm{s}$ have been realised by direct coupling of a pre-focused undulator beam into two-dimensionally confining waveguides.

Based on these results, first proof-of-principle imaging experiments were carried out (Chapter 7). A variety of samples were prepared for the different imaging regimes. Direct imaging was demonstrated with comparably large samples showing both amplitude and phase contrast. In the recorded image, amplitude objects simply produce amplitude contrast like in classical X-ray radiography, while phase objects are recognised by strongly pronounced edges.

Since absorption of small objects is low, mainly phase contrast images have been obtained in the holographic regime. Reconstruction from in-line holograms enabled the identification of small features down to a spatial resolution of $\approx 360 \mathrm{~nm}$. Micron-sized $\mathrm{Cu}$ islands were imaged to illustrate potential applications in the investigation of small clusters or nanodots. However, as expected from theory, the twin image means that reconstruction from in-line holograms is accompanied by significant artifacts. To eliminate the disturbing twin-image background, off-axis holography was demonstrated. Based on the presented Y double waveguides, a two-beam Gabor microscope was realised. The method was tested with a small tungsten tip as a sample. Besides low noise, no artifacts are visible in the reconstruction, and image quality is much better than obtained from in-line holograms. Although spatial resolution could not directly be determined by resolving correspondingly small structures, sharpness of the edges in the reconstructed phase image indicates a spatial resolution of $\approx 100 \mathrm{~nm}$. This is in approximate agreement with expectations based on the waveguide core dimensions.

Note that the numerical reconstruction from an off-axis hologram gives direct access to the phase of the optical transmission function related to the sample. This was verified by comparing the reconstructed phase shift to simulated data. Conversely, most other microscopy techniques provide phase contrast.

In summary, we conclude that X-ray waveguides indeed enhance spatial resolution in hard-X-ray microscopy, in particular in lensless full-field imaging. Besides compound refractive lenses $\left[\mathrm{SKP}^{+} 05\right]$ and mirror optics $\left[\mathrm{MMY}^{+} 05\right]$, two-dimensionally confining waveguides provide the smallest beam dimensions published so far for hard X-rays. Moreover, the beam dimensions provided by waveguides are independent of the coherence properties of the incident beam, i. e. of the size of the source. Fabrication is comparably easy and the devices are easily adapted to a specific photon energy by simply adapting their length. As shown in Chapter 2, the fundamental limit of spatial resolution is practically the same as for other X-ray focusing devices*. Hence, the advantages of X-ray waveguides are mainly down to technical reasons rather than to fundamental physical limitations.

With the advent of more brilliant sources, comparable or even higher flux can be expected from waveguides with further decreased core diameters. The X-ray free-electron laser [FAH05] can provide pulses with a duration of $\approx 100$ fs containing about $10^{12}$ trans-

*Note the possible exceptions mentioned in Chapter 2 . 
versely coherent photons MT01. This potentially enables waveguide-based lensless fullfield imaging within a single shot. Utilising appropriate materials, i. e. cladding materials of high electron density and ideally a vacuum guiding core, the cross-sectional dimensions and thus spatial resolution may approach a fundamental limit slightly below $10 \mathrm{~nm}$. Therefore, new methods of waveguide fabrication probably have to be developed, but cross-sectional dimensions of about $20 \mathrm{~nm}$ seem feasible with the presented technology, for instance by replacing Si with Ge, which provides a significantly higher electron density.

Based on the present results, waveguide-based imaging may be extended to waveguidebased tomography. Due to the small numerical aperture in the order of milliradians, the focal depth in waveguide-based hard-X-ray imaging is about 1,000 times larger than lateral spatial resolution. This in principle allows three-dimensional imaging of accordingly thick samples with practically the same spatial resolution as obtained in two-dimensional imaging.

Besides improving lensless full-field imaging microscopy, X-ray waveguides may also serve as small sources for scanning X-ray microscopy. This could for instance drastically increase spatial resolution in hard-X-ray micro-diffraction or micro-fluorescence. Moreover, all these techniques could be combined in a single instrument. By simply changing the distance between the waveguide and the sample, such an instrument could easily switch between full-field imaging and scanning mode.

Thus, it becomes possible to enhance various X-ray microscopy techniques by means of X-ray waveguides. This thesis has presented the theoretical background of both wave propagation in waveguides and waveguide-based lensless imaging. Waveguides suited for application have been fabricated and tested, and the feasibility of waveguide-based imaging was demonstrated by proof-of-principle experiments. Finally, we have developed a new kind of X-ray microscopy - two-beam Gabor microscopy with hard X-rays. 


\section{Bibliography}

[ANM01] J. Als-Nielsen and D. McMorrow, Elements of modern x-ray physics, Wiley, New York, 2001.

[Bae52] A. V. Baez, A study in diffraction microscopy with special reference to $x$-rays, J. Opt. Soc. Am. 42 (1952), 756-762.

$\left[\mathrm{BDD}^{+} 02\right] \quad$ J. H. H. Bongaerts, C. David, M. Drakopoulos, M. J. Zwanenburg, G. H. Wegdam, T. Lackner, H. Keymeulen, and J. F. van der Veen, Propagation of a partially coherent focused $x$-ray beam within a planar $x$-ray waveguide, J. Synchrotron Rad. 9 (2002), 383-393.

[BEW05] D. H. Bilderback, P. Elleaume, and E. Weckert, Review of third and next generation synchrotron light sources, J. Phys. B 38 (2005), S773-S797.

[BHT94] D. H. Bilderback, S. A. Hoffman, and D. J. Thiel, Nanometer spatialresolution achieved in hard $x$-ray-imaging and Laue diffraction experiments, Science 263 (1994), 201-203.

[BKvdV03] C. Bergemann, H. Keymeulen, and J. F. van der Veen, Focusing x-ray beams to nanometer dimensions, Phys. Rev. Lett. 91 (2003), 204801.

[BW87] M. Born and E. Wolf, Principles of optics, 6th ed., Pergamon Press, Oxford, 1987.

[CA93] W. Chen and H. Ahmed, Fabrication of 5-7 nm wide etched lines in silicon using $100 \mathrm{keV}$ electron-beam lithography and polymethylmethacrylate resist, Appl. Phys. Lett. 62 (1993), 1499-1451.

[CH94] D. C. Chrisey and G. K. Hubler (eds.), Pulsed laser deposition of thin films, Wiley, New York, 1994.

$\left[\mathrm{CHL}^{+} 05\right]$ W. Chao, B. D. Harteneck, J. A. Liddle, E. H. Anderson, and D. T. Attwood, Soft x-ray microscopy at a spatial resolution better than $15 \mathrm{~nm}$, Nature 435 (2005), 1210-1213.

[Clo99] P. Cloetens, Contribution to Phase Contrast Imaging, Reconstruction and Tomography with Hard Synchrotron Radiation, Ph.D. thesis, Vrije Universiteit Brussel and European Synchrotron Radiation Facility, 1999. 
[CLS $\left.{ }^{+} 04\right] \quad$ A. Cedola, S. Lagomarsino, F. Scarinci, M. Servidori, and V. Stanic, X-ray characterization of Si microstructures with high spatial resolution, J. Appl. Phys. 95 (2004), 1662-1666.

[Cow81] J. M. Cowley, Diffraction physics, second ed., North Holland Publishing Company, Amsterdam, New York, Oxford, 1981.

[DBR05] R. J. Davies, M. Burghammer, and C. Riekel, Studying morphological variations across single fibres using an $x$-ray waveguide, J. Synchrotron Rad. 12 (2005), 765-771.

$\left[\mathrm{DEH}^{+} 04\right] \quad$ R. B. Doak, Y. Ekinci, B. Holst, J. P. Toennies, T. Al-Kassab, and A. Heinrich, Field ionization detection of supersonic molecular beams, Rev. Sci. Instrum. 75 (2004), 405-414.

[DH99] C. David and D. Hambach, Line width control using a low voltage electron beam, Microelectron. Eng. 46 (1999), 219-222.

$\left[\mathrm{DRH}^{+} 02\right] \quad$ Y. Dabin, G. Rostaing, O. Hignette, A. Rommeveaux, and A. K. Freund, The present state of Kirkpatrick-Baez mirror systems at the ESRF, Proceedings of SPIE 4782 (2002), 235-245.

[ELE $\left.{ }^{+} 04\right] \quad$ S. Eisebitt, M. Lörgen, E. Eberhardt, J. Lüning, S. Andrews, and J. Stöhr, Scalable approach for lensless imaging at $x$-ray wavelengths, Phys. Rev. Lett. 84 (2004), 3373-2275.

[ELS ${ }^{+}$04] S. Eisebitt, J. Lüning, W. F. Schlotter, M. Lörgen, O. Hellwig, W. Eberhardt, and J. Stöhr, Lensless imaging of magnetic nanostructures by $x$-ray spectroholography, Nature 432 (2004), 885-888.

[ES52] H. M. A. El-Sum, Reconstructed wavefront microscopy, Ph.D. thesis, Standford University, 1952.

[ESK52] H. M. A. El-Sum and P. Kirkpatrick, Microscopy by reconstructed wavefronts, Phys. Rev. 85 (1952), 763.

[FAH05] J. Feldhaus, J. Arthur, and J. B. Hastings, X-ray free-electron lasers, J. Phys. B 38 (2005), S799-S819.

[Fie78] R. J. Fienup, Reconstruction of an object from the modulus of its Fourier transform, Opt. Lett. 3 (1978), 27-29.

$\left[\mathrm{FJO}^{+} 04\right] \quad$ C. Fuhse, A. Jarre, C. Ollinger, J. Seeger, T. Salditt, and R. Tucoulou, Frontcoupling of a pre-focused $x$-ray beam into a mono-modal planar waveguide, Appl. Phys. Lett. 85 (2004), 1907-1909. 
[Foc65] V. A. Fock, Electromagnetic diffraction and propagation problems, Pergamon Press, Oxford, London, Edinburgh, New York, Paris, Frankfurt, 1965.

[FOOM96] J. Fujita, Y. Ohnishi, Y. Ochiai, and S. Matsui, Ultrahigh resolution of calixarene negative resist in electron beam lithography, Appl. Phys. Lett. 68 (1996), 1297-1299.

[FS05] C. Fuhse and T. Salditt, Finite-difference field calculations for onedimensionally confined x-ray waveguides, Physica B 357 (2005), 57-60.

[FSD $\left.{ }^{+} 93\right] \quad$ Y. P. Feng, S. K. Sinha, H. W. Deckman, J. B. Hastings, and D. P. Siddons, $X$-ray flux enhancement in thin-film waveguides using resonant beam couplers, Phys. Rev. Lett. 71 (1993), 537-540.

[FSF $\left.{ }^{+} 95\right] \quad$ Y. P. Feng, S. K. Sinha, E. E. Fullerton, G. Grübel, D. Abernathy, D. P. Siddons, and J. B. Hastings, X-ray Fraunhofer diffraction patterns from a thin-film waveguide, Appl. Phys. Lett. 67 (1995), 3647-3649.

[Gab48] D. Gabor, A new microscopic principle, Nature 161 (1948), 777-778.

[Gab49] D. Gabor, Microscopy by reconstructed wave-fronts, Proc. R. Soc. London, Ser. A 197 (1949), 454-487.

[Glo71] D. Gloge, Weakly guiding fibers, Appl. Opt. 10 (1971), 2252-2258.

[Glo79] D. Gloge, The optical fibre as a transmission medium, Rep. Prog. Phys. 42 (1979), 1777-1824.

[GNR $\left.{ }^{+} 03\right]$ P. Guttmann, B. Niemann, S. Rehbein, C. Knöchel, D. Rudolph, and G. Schmahl, The transmission x-ray microscope at BESSY II, J. Phys. IV 104 (2003), 85-90.

[Goo68] J. W. Goodman, Introduction to Fourier optics, McGraw-Hill Book Company, New York, 1968.

$\left[\mathrm{HCL}^{+} 03\right]$ O. Hignette, P. Cloetens, W.-K. Lee, W. Ludwig, and G. Rostaing, Hard $x$-ray microscopy with reflecting mirrors status and perspective of the ESRF technology, J. Phys. IV 104 (2003), 231-234.

$\left[\mathrm{HCR}^{+}\right.$05] O. Hignette, P. Cloetens, G. Rostaing, P. Bernard, and C. Morawe, Efficient sub 100 nm focusing of hard $x$ rays, Rev. Sci. Instrum. 76 (2005), 063709.

[Hec89] E. Hecht, Optics, second ed., Addison-Wesley, Reading, Massachusetts, 1989. 
[HGD93] B. L. Henke, E. M. Gullikson, and J. C. Davis, X-ray interactions: photoabsorption, scattering, transmission, and reflection at $E=50-30000 \mathrm{eV}, Z=1$ 92, Atomic Data and Nuclear Data Tables 54 (1993), 181-342, available from http://www.cxro.lbl.gov/optical_constants.

[HJK $\left.{ }^{+} 87\right]$ M. Howells, C. Jacobsen, J. Kirz, R. Feder, K. McQuaid, and S. Rothman, $X$-ray holograms at improved resolution: a study of zymogen granules, Science 238 (1987), 514-517.

[How84] M. R. Howells, Possibilities of x-ray holography using synchrotron radiation, X-Ray Microscopy (G. Schmahl and D. Rudolph, eds.), Springer Series in Optical Sciences, vol. 43, Springer-Verlag, Berlin, 1984, pp. 318-335.

[HTB94] S. A. Hoffman, D. J. Thiel, and D. H. Bilderback, Developments in tapered monocapillary and polycapillary glass x-ray concentrators, Nucl. Intrum. Methods A 347 (1994), 384-389.

$\left[\mathrm{IFO}^{+} 03\right]$ M. Ishida, J. Fujita, T. Ogura, Y. Ochiai, E. Ohshima, and J. Momoda, Sub-10-nm-scale lithography using p-chloromethyl-methoxy-calix[4]arene resist, Jpn. J. Appl. Phys. 42 (2003), 3913-3916.

[Jar05] A. Jarre, Hard x-ray waveguide optics, Ph.D. thesis, Georg-AugustUniversität Göttingen, 2005.

$\left[\mathrm{JCF}^{+} 01\right] \quad$ W. Jark, A. Cedola, S. D. Fonzo, M. Fiordelisi, S. Lagomarsino, N. V. Kovalenko, and V. A. Chernov, High gain beam compression in new-generation thin-film x-ray waveguides, Appl. Phys. Lett. 78 (2001), 1192-1194.

$\left[\mathrm{JFO}^{+} 05\right] \quad$ A. Jarre, C. Fuhse, C. Ollinger, J. Seeger, R. Tucoulou, and T. Salditt, Twodimensional hard $x$-ray beam compression by combined focusing and waveguide optics, Phys. Rev. Lett. 94 (2005), 074801.

[JHKR90] C. Jacobsen, M. Howells, J. Kirz, and S. Rothman, X-ray holographic microscopy using photoresists, J. Opt. Soc. Am. A 7 (1990), 1847-1861.

$\left[\mathrm{JLG}^{+} 00\right] \quad$ S. D. F. W. Jark, S. Lagomarsino, C. Giannini, L. D. Caro, A. Cedola, and M. Müller, Non-destructive determination of local strain with 100-nanometre spatial resolution, Nature 403 (2000), 638-640.

$\left[\mathrm{JSP}^{+} 04\right] \quad$ A. Jarre, T. Salditt, T. Panzner, U. Pietsch, and F. Pfeiffer, White beam x-ray waveguide optics, Appl. Phys. Lett. 85 (2004), 161-163.

[KB48] P. Kirkpatrick and A. V. Baez, Formation of optical images by x-rays, J. Opt. Soc. Am. 38 (1948), 766-774. 
$\left[\mathrm{KEL}^{+} 04\right] \quad$ S. A. Knaack, J. Eddington, Q. Leonard, F. Cerrina, and M. Onellion, Dense arrays of nanopores as x-ray lithography masks, Appl. Phys. Lett. 84 (2004), 3388-3390.

[KJH95] J. Kirz, C. Jacobsen, and M. Howells, Soft x-ray microscopes and their biological application, Q. Rev. Biophys. 28 (1995), 33-130.

[KK03] P. Karimov and E. Z. Kurmaev, Application of generic algorithms for optimization of x-ray waveguides, Phys. Lett. A 320 (2003), 234-237.

[KPV95] Y. V. Kopylov, A. V. Popov, and A. V. Vinogradov, Application of the parabolic wave equation to x-ray diffraction optics, Optics Commun. 118 (1995), 619-636.

[KPV96] Y. V. Kopylov, A. V. Popov, and A. V. Vinogradov, Diffraction phenomena inside thick fresnel zone plates, Radio Sci. 31 (1996), 1815-1822.

$\left[\mathrm{KS}^{+} 04\right] \quad$ Y. Kohmura, T. Sakurai, , T. Ishikawa, and Y. Suzuki, Phase retrieval with two-beam off-axis x-ray holography, J. Appl. Phys. 96 (2004), 1781-1784.

$\left[\mathrm{KWF}^{+} 03\right]$ H. U. Krebs, M. Weisheit, J. Faupel, E. Süske, T. Scharf, C. Fuhse, M. Störmer, K. Sturm, M. Seibt, H. Kijewski, D. Nelke, E. Panchenko, and M. Buback, Pulsed laser deposition (PLD) - a versatile thin film technique, Adv. Sol. Stat. Phys. 43 (2003), 505-518.

$\left[\mathrm{LBM}^{+}\right.$06] S. Lagomarsino, I. Bukreeva, V. Mocella, A. Surpi, T. Bigault, and A. Cedola, Large-distance refocusing of a submicrometre beam from an x-ray waveguide, J. Synchrotron Rad. 13 (2006), 85-87.

$\left[\mathrm{LCC}^{+}\right.$97] S. Lagomarsino, A. Cedola, P. Cloetens, S. D. Fonzo, W. Jark, S. Souillié, and C. Riekel, Phase contrast hard $x$-ray microscopy with submicron resolution, Appl. Phys. Lett. 71 (1997), 2557-2559.

[Lei97] Leica Lithograhie Systeme Jena GmbH, Jena, Germany, LEICA LION LV 1 - Operator Manual, 1.0 ed., 1997.

[LHJK96] S. Lindaas, M. Howells, C. Jacobsen, and A. Kalinovski, X-ray holographic microscopy by means of photoresist recording and atomic-force microscope readout, J. Opt. Soc. Am. A 13 (1996), 1788-1800.

[LK03] W. Lauterborn and T. Kurz, Coherent optics, 2nd ed., Springer, Berlin, 2003.

[LL79] L. D. Landau and E. M. Lifschitz, Quantenmechanik, 6th ed., Lehrbuch der theoretischen Physik, vol. 3, Akademie-Verlag, Berlin, 1979. 
[LLT97] S. G. Lipson, H. S. Lipson, and D. S. Tannhauser, Optik, Springer, Berlin, 1997.

$\left[\mathrm{LPB}^{+} 05\right] \quad$ D. Loidl, O. Paris, M. Burghammer, C. Riekel, and H. Peterlik, Direct observation of nanocrystallite buckling in carbon fibers under bending load, Phys. Rev. Lett. 95 (2005), 225501.

[LSK $\left.{ }^{+} 05\right] \quad$ B. Lengeler, C. G. Schroer, M. Kuhlmann, B. Benner, T. F. Günzler, O. Kurapova, F. Zontone, A. Snigirev, and I. Snigireva, Refractive $x$-ray lenses, J. Phys. D 38 (2005), A218-A222.

[LU62] E. N. Leith and J. Upatnieks, Reconstructed wavefronts and communication theory, J. Opt. Soc. Am. 52 (1962), 1123-1130.

[LU63] E. N. Leith and J. Upatnieks, Wavefront reconstruction with continuous-tone objects, J. Opt. Soc. Am. 53 (1963), 1377-1381.

[LUH65] E. N. Leith, J. Upatnieks, and K. A. Haines, Microscopy by wavefront reconstruction, J. Opt. Soc. Am. 55 (1965), 981-986.

[Mad02] M. J. Madou, Fundamentals of microfabrication, CRC Press LLC, Boca Raton, London, New York, Washington, D.C., 2002.

[Mar71] D. Marcuse, Bending losses of the asymmetric slab waveguide, Bell Syst. Tech. J. 50 (1971), 2551-2563.

[Mar74] D. Marcuse, Theory of dielectric optical waveguides, Academic Press, New York and London, 1974.

[MCKS99] J. Miao, P. Charalambous, J. Kirz, and D. Sayre, Extending the methodology of x-ray crystallography to allow imaging of micrometre-sized non-crystalline specimens, Nature 400 (1999), 342-344.

[Med94] R. Medenwaldt, Development of $x$-ray microscopy in aarhus and ultra thin foil production to speed up high resolution x-ray optics, Ph.D. thesis, Aarhus University, 1994.

[MH95] R. Medenwaldt and M. Hettwer, Production of ultra thin silicon foils, J. X-ray Sci. Technol. 5 (1995), 202-206.

$\left[\mathrm{MHK}^{+} 05\right] \quad$ D. M. Mills, J. R. Helliwell, A. Kvick, T. Ohta, I. A. Robinson, and A. Authier, Brightness, spectral brightness or brilliance - Report of the Working Group on Synchrotron Radiation Nomenclature, Journal of Synchrotron Radiation 12 (2005), 385. 
[MIAH03] J. Miao, T. Ishikawa, E. H. Anderson, and K. O. Hodgson, Phase retrieval of diffraction patterns from noncrystalline samples using the oversampling method, Phys. Rev. B 67 (2003), 174104.

$\left[\mathrm{MKJ}^{+}\right.$92] I. McNulty, J. Kirz, C. Jacobsen, E. H. Anderson, M. R. Howells, and D. P. Kern, High-resolution imaging by Fourier transform x-ray holography, Science 256 (1992), 1009-1012.

$\left[\mathrm{MMW}^{+} 02\right]$ S. C. Mayo, P. R. Miller, S. W. Wilkins, J. T. Davis, D. Gao, T. E. Gureyev, D. Paganin, D. J. Parry, A. Pogany, and A. W. Stevenson, Quantitative x-ray projection microscopy: phase-contrast and multi-spectral imaging, J. Microsc. 207 (2002), 79-96.

$\left[\mathrm{MMY}^{+} 05\right]$ H. Mimura, S. Matsuyama, H. Yumoto, H. Hara, K. Yamamura, Y. Sano, M. Shibahara, K. Endo, Y. Mori, Y. Nishino, K. Tamasaku, M. Yabashi, T. Ishikawa, and K. Yamauchi, Hard x-ray diffraction-limited nanofocusing with Kirckpartick-Beaz mirrors, Jpn. J. Appl. Phys. 44 (2005), L539-L542.

[MT01] G. Materlik and T. Trentscher (eds.), TESLA Technical Design Report: Part V. The X-Ray Free-Electron Laser, Deutsches Elektronen-Synchrotron (DESY), Hamburg, Germany, 2001.

[OFJS05] C. Ollinger, C. Fuhse, A. Jarre, and T. Salditt, Two-dimensional x-ray waveguide on a grating, Physica B 357 (2005), 53-56.

[Ollon] C. Ollinger, Ph.D. thesis, Georg-August-Universität Göttingen, in preparation.

$\left[\mathrm{PDB}^{+} 02\right]$ F. Pfeiffer, C. David, M. Burghammer, C. Riekel, and T. Salditt, Twodimensional x-ray waveguides and point sources, Science 297 (2002), 230234.

[Pfe02] F. Pfeiffer, X-ray and neutron waveguides, Ph.D. thesis, Universität des Saarlandes, 2002.

[PGW97] A. Pogany, D. Gao, and S. W. Wilkins, Contrast and resolution in imaging with a microfocus x-ray source, Rev. Sci. Instrum. 68 (1997), 2774-2782.

[PJB $\left.{ }^{+} 05\right] \quad$ M. Poulsen, F. Jensen, O. Bunk, R. Feidenhans'l, and D. W. Breiby, Silicon waveguides produced by wafer bonding, Appl. Phys. Lett. 87 (2005), 261904.

$\left[\mathrm{PLG}^{+} 03\right]$ T. Panzner, W. Leitenberger, J. Grenzer, Y. Bodenthin, T. Geue, U. Pietsch, and H. Möhwald, Coherence experiments at the energy-dispersive reflectometry beamline at BESSY II, J. Phys. D 36 (2003), A93-A97. 
[PR55] D. W. Peaceman and H. H. Rachford, The numerical solution of parabolic and elliptic differential equations, J. Soc. Indust. Appl. Math. 3 (1955), 28-41.

[Pre92] W. H. Press, Numerical recipes in $C$, 2nd ed., Cambridge University Press, Cambridge, 1992.

[PSD02] F. Pfeiffer, T. Salditt, and C. David, Reflection of waveguided $x$-rays in twodimensional nanostructures, J. Appl. Crystallogr. 35 (2002), 430-433.

[PV98] V. V. Protopopov and K. A. Valiev, Theory of an ideal compound $x$-ray lens, Optics Commun. 151 (1998), 297-312.

[Ree87] W. G. Rees, The validity of the Fresnel approximation, Eur. J. Phys. 8 (1987), $44-48$.

[Rei98] L. Reimer, Scanning electron microscopy, Springer-Verlag, Berlin, Heidelberg, New York, 1998.

[See04] J. Seeger, Nanolithographische Herstellung von 2D-Wellenleitern für harte Röntgenstrahlung, diploma thesis, Georg-August-Universität Göttingen, 2004.

[SFO ${ }^{+99}$ J. Sone, J. Fujita, Y. Ochiai, S. Manako, S. Matsui, E. Nomura, T. Baba, H. Kawaura, T. Sakamoto, C. D. Chen, Y. Nakamura, and J. S. Tsai, Nanofabrication towards sub-10 nm and its application to novel nanodevices, Nanotechnology 10 (1999), 135-141.

[SJP03] J. Susini, D. Joyeux, and F. Polack (eds.), X-ray microscopy 2002, Proceedings of the 7th International Conference on X-ray Microscopy, ESRF, Grenoble, 2003.

$\left[\mathrm{SKP}^{+} 05\right] \quad$ C. G. Schroer, O. Kurapova, J. Patommel, P. Boye, J. Feldkamp, B. Lengeler, M. Burhammer, C. Riekel, L. Vincze, A. van der Hart, and M. Küchler, Hard $x$-ray nanoprobe based on refractive x-ray lenses, Appl. Phys. Lett. 87 (2005), 124103.

[SKSL96] A. Snigirev, V. Kohn, I. Snigireva, and B. Lengeler, A compound refractive lens for focusing high-energy x-rays, Nature 384 (1996), 49-51.

[SL05] C. G. Schroer and B. Lengeler, Focusing hard $x$ rays to nanometer dimensions by adiabatically focusing lenses, Phys. Rev. Lett. 94 (2005), 054802.

[Sni61] E. Snitzer, Cylindrical dielectric waveguide modes, J. Opt. Soc. Am. 51 (1961), 491-498. 
[SO91] R. Scarmozzino and R. M. Osgood, Comparison of finite-difference and Fourier-transform solutions of the parabolic wave equation with emphasis on integrated-optics applications, J. Opt. Soc. Am. A 8 (1991), 724-731.

[SS73] E. Spiller and A. Segmüller, Propagation of $x$ rays in waveguides, Appl. Phys. Lett. 24 (1973), 60-61.

[SS06] I. Snigireva and A. Snigirev, X-ray microanalytical techniques based on synchrotron radiation, J. Environ. Monit. 8 (2006), 33-42.

[SSKS95] A. Snigirev, I. Snigireva, V. K. S. Kuznetsov, and I. Schelokov, On the possibilities of $x$-ray phase contrast microimaging by coherent high-energy synchrotron radiation, Rev. Sci. Instrum. 66 (1995), 5486-5492.

$\left[\mathrm{SSS}^{+} 03\right]$ L. G. Shabel'nikov, A. Snigirev, I. Snigireva, S. Kuznetsov, V. Yunkin, V. Levashov, and M. Grigoriev, X-ray refractive planar lenses in combination with planar x-ray waveguide, J. Phys. IV 104 (2003), 227-230.

[ST91] B. E. A. Saleh and M. C. Teich, Fundamentals of photonics, Wiley, New York, 1991.

[STMC $\left.{ }^{+} 05\right]$ A. Somogyi, R. Tucoulou, G. Martinez-Criado, A. Homs, J. Cauzid, P. Bleuet, S. Bohic, and A. Simionovici, ID22: a multitechnique hard $x$-ray microprobe beamline at the European Synchrotron Radiation Facility, J. Synchrotron Rad. 12 (2005), 208-215.

[Suz04] Y. Suzuki, Resolution limit of refractive lens and Fresnel lens in x-ray region, Jpn. J. Appl. Phys. 43 (2004), 7311-7314.

[Tho95] J. W. Thomas, Numerical partial differential equations, Springer-Verlag, New York, Berlin, Heidelberg, 1995.

[Tie77] P. K. Tien, Integrated optics and new wave phenomena in optical waveguides, Rev. Mod. Phys. 49 (1977), 361-454.

[Vog97] H. Vogel, Gerthsen Physik, 19 ed., Springer, Berlin, 1997.

[Wei02] T. Weitkamp, Imaging and Tomography with High Resolution Using Coherent Hard Synchrotron Radiation, Ph.D. thesis, Universität Hamburg, 2002.

[Wei03] E. W. Weisstein, MathWorld - A Wolfram Web Resource, http:// mathworld.wolfram.com, and CRC Concise Encyclopedia of Mathematics, 2nd ed., CRC Press LLC, Boca Raton, 2003.

[Win98] D. L. Windt, IMD-software for modeling the optical properties of multilayer films, Comput. Phys. 12 (1998), 360-370. 
[WSTA97] N. Watanabe, K. Sakurai, A. Takeuchi, and S. Aoki, Soft-x-ray Gabor holography by use of a backilluminated CCD camera, Appl. Opt. 36 (1997), 74337436.

[Yeh90] C. Yeh, Handbook of Fiber Optics, Academic Press, San Diego, 1990.

$\left[\mathrm{ZBP}^{+} 00\right] \quad$ M. J. Zwanenburg, J. H. H. Bongaerts, J. F. Peters, D. Riese, and J. F. van der Veen, Focusing of coherent x-rays in a tapered planar waveguide, Physica B 283 (2000), 285-288.

$\left[\mathrm{ZPB}^{+} 99\right] \quad$ M. J. Zwanenburg, J. F. Peters, J. H. H. Bongaerts, S. A. de Vries, D. L. Abernathy, and J. F. van der Veen, Coherent propagation of $x$ rays in a planar waveguide with a tunable air gap, Phys. Rev. Lett. 82 (1999), 1696-1699.

[ZvdVFN00] M. J. Zwanenburg, J. F. van der Veen, H. G. Ficke, and H. Neerings, A planar $x$-ray waveguide with a tunable air gap for the structural investigation of confined fluids, Rev. Sci. Instrum. 71 (2000), 1723-1732. 


\section{A Appendix}

\section{A.1 The Fourier transform}

This section briefly summarises some well-known properties of the Fourier transform. We only state the theorems relevant for this work and refer the reader to standard textbooks Goo68, Wei03, for more detail and proof.

Definition: The two-dimensional Fourier transform of a function $f(x, y)$ is defined by [LK03]

$$
\begin{aligned}
\tilde{f}\left(\nu_{x}, \nu_{y}\right) & :=\mathcal{F}[f(x, y)]\left(\nu_{x}, \nu_{y}\right) \\
& :=\int f(x, y) \exp \left[-2 \pi i\left(\nu_{x} x+\nu_{y} y\right)\right] \mathrm{d} x \mathrm{~d} y .
\end{aligned}
$$

The variables $\nu_{x}$ and $\nu_{y}$ are called spatial frequencies. In X-ray scattering and diffraction, it is more common to use the corresponding components $k_{x}$ and $k_{y}$ of the wave vector $\boldsymbol{k}$ with $k_{x}=2 \pi \nu_{x}$ and $k_{y}=2 \pi \nu_{y}$.

Differentiation: The operator $\partial / \partial x$ in real space corresponds to a multiplication with $2 \pi i \nu_{x}$ in Fourier space [LK03]:

$$
\mathcal{F}\left[\frac{\partial}{\partial x} f(x, y)\right]\left(\nu_{x}, \nu_{y}\right)=2 \pi i \nu_{x} \mathcal{F}[f(x, y)]\left(\nu_{x}, \nu_{y}\right) .
$$

In the same way, the operator $\partial / \partial y$ corresponds to a multiplication with $2 \pi i \nu_{y}$.

Convolution Theorem: The Fourier transform of the convolution

$$
(f * g)(x, y):=\iint f\left(x^{\prime}, y^{\prime}\right) g\left(x-x^{\prime}, y-y^{\prime}\right) \mathrm{d} x^{\prime} \mathrm{d} y^{\prime}
$$

of two functions $f(x, y)$ and $g(x, y)$ is given by the product of the individual Fourier transforms:

$$
\mathcal{F}[(f * g)(x, y)]\left(\nu_{x}, \nu_{y}\right)=\mathcal{F}[f(x, y)]\left(\nu_{x}, \nu_{y}\right) \times \mathcal{F}[g(x, y)]\left(\nu_{x}, \nu_{y}\right)
$$

Crosscorrelation Theorem: The Fourier transform of the crosscorrelation

$$
(f \otimes g)(x, y):=\iint f\left(x^{\prime}, y^{\prime}\right) g^{*}\left(x^{\prime}-x, y^{\prime}-y\right) \mathrm{d} x^{\prime} \mathrm{d} y^{\prime}
$$

of two functions $f(x, y)$ and $g(x, y)$ is given by

$$
\mathcal{F}[(f \otimes g)(x, y)]\left(\nu_{x}, \nu_{y}\right)=\mathcal{F}[f(x, y)]\left(\nu_{x}, \nu_{y}\right) \times \mathcal{F}[g(x, y)]^{*}\left(\nu_{x}, \nu_{y}\right) .
$$

The symbol * denotes the complex conjugate. 
Wiener-Khinchin Theorem: The Wiener-Khinchin theorem is a special case of the crosscorrelation theorem. From Eq. A.7) we find with $f=g$ :

$$
\mathcal{F}[(f \otimes f)(x, y)]\left(\nu_{x}, \nu_{y}\right)=|\mathcal{F}[f(x, y)]|^{2}\left(\nu_{x}, \nu_{y}\right)
$$

This means that the power spectrum $|\mathcal{F}[f]|^{2}$ of a function is the Fourier transform of the autocorrelation function $f \otimes f$. Intensity measured in the far-field diffraction pattern of a waveguide corresponds to the power spectrum of the electric field at the end face of the waveguide. Accordingly, the respective autocorrelation function is obtained from an inverse Fourier transformation.

\section{A.2 Analogy between parallel-beam and point-source holography}

In this section, we discuss the analogy between holography with plane and spherical waves. In a strict sense, the waves are not exactly plane or spherical, since we have to introduce an aperture function of finite extent to ensure convergence of the respective integrals. The consideration is based on the Fresnel-Kirchhoff diffraction formula in paraxial approximation as given in Eq. (5.4). Propagation from plane $\ell$ to plane $m$ is thus described by

$$
E_{m}\left(x_{m}, y_{m}\right)=\frac{\exp (i k z)}{i \lambda z} \int E_{\ell}\left(x_{\ell}, y_{\ell}\right) \exp \left(i k \frac{\left(x_{m}-x_{\ell}\right)^{2}+\left(y_{m}-y_{\ell}\right)^{2}}{2 z}\right) \mathrm{d} x_{\ell} \mathrm{d} y_{\ell}
$$

\section{A.2.1 In-line holography}

We first consider a true point source located in the origin of plane 0 with a field given by

$$
E_{0}^{\text {point }}\left(x_{0}, y_{0}\right)=\delta\left(x_{0}, y_{0}\right)
$$

Within the paraxial approximation, the electromagnetic field incident on a sample located in a plane 1 at distance $z_{1}$ behind the centre of a spherical wave is then given by

$$
E_{1}^{\text {point }}\left(x_{1}, y_{1}\right)=\frac{\exp \left(i k z_{1}\right)}{i \lambda z_{1}} \exp \left[i k\left(\frac{x_{1}^{2}+y_{1}^{2}}{2 z_{1}}\right)\right]
$$

As shown in Section 5.2.4, the waveguide beam incident in a sample may be described as a spherical wave enveloped by the far-field diffraction pattern of the waveguide. We account for this envelope by introducing an aperture function $A\left(x_{1}, y_{1}\right)$. The calculations presented in this thesis are carried out with Gaussian aperture functions. We thus find in plane 1:

$$
\begin{aligned}
E_{1}^{\mathrm{ap}}\left(x_{1}, y_{1}\right) & =A\left(x_{1}, y_{1}\right) \times E_{1}^{\mathrm{point}}\left(x_{1}, y_{1}\right) \\
& =A\left(x_{1}, y_{1}\right) \times \frac{\exp \left(i k z_{1}\right)}{i \lambda z_{1}} \exp \left[i k\left(\frac{x_{1}^{2}+y_{1}^{2}}{2 z_{1}}\right)\right] .
\end{aligned}
$$


Let us now consider a sample with an optical transmission function $\tau\left(x_{1}, y_{y}\right)$ located in plane 1 . The electric field $E_{1}^{\prime}\left(x_{1}, y_{1}\right)$ downstream of the sample is then given by

$$
\begin{aligned}
E_{1}\left(x_{1}, y_{1}\right) & =\tau\left(x_{1}, y_{1}\right) \times E_{1}^{\mathrm{ap}}\left(x_{1}, y_{1}\right) \\
& =\tau\left(x_{1}, y_{1}\right) \times A\left(x_{1}, y_{1}\right) \times \frac{\exp \left(i k z_{1}\right)}{i \lambda z_{1}} \exp \left[i k\left(\frac{x_{1}^{2}+y_{1}^{2}}{2 z_{1}}\right)\right] .
\end{aligned}
$$

Within the paraxial approximation, the electric field incident on a detector positioned at a distance $z_{2}$ behind the sample is given by

$$
\begin{aligned}
& E_{2}\left(x_{2}, y_{2}\right)=\frac{\exp \left(i k z_{2}\right)}{i \lambda z_{2}} \int E_{1}\left(x_{1}, y_{1}\right) \exp \left[i k\left(\frac{\left(x_{2}-x_{1}\right)^{2}+\left(y_{2}-y_{1}\right)^{2}}{2 z_{2}}\right)\right] \mathrm{d} x_{1} \mathrm{~d} y_{1} \\
& =\frac{\exp \left(i k z_{2}\right)}{i \lambda z_{2}} \int \tau\left(x_{1}, y_{1}\right) A\left(x_{1}, y_{1}\right) \frac{\exp \left(i k z_{1}\right)}{i \lambda z_{1}} \exp \left[i k\left(\frac{x_{1}^{2}+y_{1}^{2}}{2 z_{1}}\right)\right] \\
& \times \exp \left[i k\left(\frac{\left(x_{2}-x_{1}\right)^{2}+\left(y_{2}-y_{1}\right)^{2}}{2 z_{2}}\right)\right] \mathrm{d} x_{1} \mathrm{~d} y_{1} \\
& =-\frac{\exp \left[i k\left(z_{1}+z_{2}\right)\right]}{\lambda^{2} z_{1} z_{2}} \int \tau\left(x_{1}, y_{1}\right) A\left(x_{1}, y_{1}\right) \\
& \times \exp \left[i k\left(\frac{x_{1}{ }^{2}+y_{1}{ }^{2}}{2 z_{1}}+\frac{\left(x_{2}-x_{1}\right)^{2}+\left(y_{2}-y_{1}\right)^{2}}{2 z_{2}}\right)\right] \mathrm{d} x_{1} \mathrm{~d} y_{1} \\
& =-\frac{\exp \left[i k\left(z_{1}+z_{2}\right)\right]}{\lambda^{2} z_{1} z_{2}} \int \tau\left(x_{1}, y_{1}\right) A\left(x_{1}, y_{1}\right) \\
& \times \exp \left[i k\left(\frac{z_{2}\left(x_{1}^{2}+y_{1}^{2}\right)+z_{1}\left(\left(x_{2}-x_{1}\right)^{2}+\left(y_{2}-y_{1}\right)^{2}\right)}{2 z_{1} z_{2}}\right)\right] \mathrm{d} x_{1} \mathrm{~d} y_{1} \\
& =-\frac{\exp \left[i k\left(z_{1}+z_{2}\right)\right]}{\lambda^{2} z_{1} z_{2}} \int \tau\left(x_{1}, y_{1}\right) A\left(x_{1}, y_{1}\right) \\
& \times \exp \left[i k \frac{\left(\sqrt{z_{1}+z_{2}} x_{1}-\frac{z_{1}}{\sqrt{z_{1}+z_{2}}} x_{2}\right)^{2}+z_{1} x_{2}{ }^{2}-\left(\frac{z_{1}}{\sqrt{z_{1}+z_{2}}} x_{2}\right)^{2}}{2 z_{1} z_{2}}\right] \\
& \times \exp \left[i k \frac{\left(\sqrt{z_{1}+z_{2}} y_{1}-\frac{z_{1}}{\sqrt{z_{1}+z_{2}}} y_{2}\right)^{2}+z_{1} y_{2}^{2}-\left(\frac{z_{1}}{\sqrt{z_{1}+z_{2}}} y_{2}\right)^{2}}{2 z_{1} z_{2}}\right] \mathrm{d} x_{1} \mathrm{~d} y_{1} \\
& =-\frac{\exp \left[i k\left(z_{1}+z_{2}\right)\right]}{\lambda^{2} z_{1} z_{2}} \exp \left[i k \frac{z_{1} x_{2}^{2}-\left(\frac{z_{1}}{\sqrt{z_{1}+z_{2}}} x_{2}\right)^{2}+z_{1} y_{2}^{2}-\left(\frac{z_{1}}{\sqrt{z_{1}+z_{2}}} y_{2}\right)^{2}}{2 z_{1} z_{2}}\right] \\
& \times \int \tau\left(x_{1}, y_{1}\right) A\left(x_{1}, y_{1}\right) \exp \left[i k \frac{\left(\sqrt{z_{1}+z_{2}} x_{1}-\frac{z_{1}}{\sqrt{z_{1}+z_{2}}} x_{2}\right)^{2}}{2 z_{1} z_{2}}\right] \\
& \times \exp \left[i k \frac{\left(\sqrt{z_{1}+z_{2}} y_{1}-\frac{z_{1}}{\sqrt{z_{1}+z_{2}}} y_{2}\right)^{2}}{2 z_{1} z_{2}}\right] d x_{1} d y_{1} \\
& =-\frac{\exp \left[i k\left(z_{1}+z_{2}+\frac{z_{1}\left(z_{1}+z_{2}\right)\left(x_{2}{ }^{2}+y_{2}{ }^{2}\right)-z_{1}^{2}\left(x_{2}{ }^{2}+y_{2}{ }^{2}\right)}{2 z_{1} z_{2}\left(z_{1}+z_{2}\right)}\right)\right]}{i \lambda^{2} z_{1} z_{2}} \int \tau\left(x_{1}, y_{1}\right) A\left(x_{1}, y_{1}\right) \\
& \times \exp \left[i k \frac{\left(z_{1}+z_{2}\right)\left(x_{1}-\frac{z_{1}}{z_{1}+z_{2}} x_{2}\right)^{2}+\left(z_{1}+z_{2}\right)\left(y_{1}-\frac{z_{1}}{z_{1}+z_{2}} y_{2}\right)^{2}}{2 z_{1} z_{2}}\right] \mathrm{d} x_{1} \mathrm{~d} y_{1} \text {. }
\end{aligned}
$$


With the magnification factor

$$
M=\frac{z_{1}+z_{2}}{z_{1}}
$$

and the effective defocusing distance

$$
z_{\mathrm{eff}}=\frac{z_{1} z_{2}}{z_{1}+z_{2}}
$$

we find

$$
\begin{aligned}
E_{2}\left(x_{2}, y_{2}\right)= & -\frac{\exp \left[i k\left(\left(z_{1}+z_{2}\right)+\frac{x_{2}^{2}+y_{2}^{2}}{2\left(z_{1}+z_{2}\right)}\right)\right]}{i \lambda^{2} z_{1} z_{2}} \\
& \times \int \tau\left(x_{1}, y_{1}\right) A\left(x_{1}, y_{1}\right) \times \exp \left[i k \frac{\left(x_{1}-\frac{x_{2}}{M}\right)^{2}+\left(y_{1}-\frac{y_{2}}{M}\right)^{2}}{2 z_{\text {eff }}}\right] \mathrm{d} x_{1} \mathrm{~d} y_{1} .
\end{aligned}
$$

Apart from a separable factor, the intensity observed at the position $\left(x_{2}, y_{2}\right)$ on the detector is thus equivalent to the intensity which one would observe at the position $(x / M, y / M)$ on a detector situated at a distance $z_{\text {eff }}$, if the object was illuminated by a plane wave with the same aperture function $A\left(x_{1}, y_{1}\right)$. The problem of point source illumination is thus reduced to the case of parallel beam illumination, which is much more quickly carried out numerically using fast Fourier transformation algorithms [Pre92].

Numerical reconstruction of a measured hologram is therefore carried out as follows: The recorded image is scaled down by the factor $M$. The hologram is then illuminated by a plane wave, i. e. the electric field amplitude in the hologram plane is assumed to be proportional to the measured intensity. Finally, this field is propagated over the effective defocusing distance $z_{\text {eff }}$.

\section{A.2.2 Off-axis holography}

We may extend the arguments given for in-line holography to the case of off-axis holography by heuristic arguments. Consider the recorded diffraction pattern when there is no sample in the beam. As shown in Section 6.9.3, the pattern is given by a waveguide far-field diffraction pattern multiplied by a Young pattern. In the small angle approximation the Young fringes are spaced by an angle

$$
\alpha_{Y} \approx \sin \alpha_{Y}=\lambda / d_{s}
$$

The corresponding distance in plane 2 is given by

$$
Y_{2}=\alpha_{Y} z_{2}
$$

After demagnification by $M$, the fringe spacing of the hologram used for reconstruction is

$$
Y_{\mathrm{eff}}=\alpha_{Y} z_{2} / M=\frac{\lambda z_{\mathrm{eff}}}{d_{s}}
$$


This corresponds to the interference pattern of two beams crossing at angle

$$
\theta_{\mathrm{eff}}=\frac{d_{S}}{z_{\mathrm{eff}}}
$$

Numerical reconstruction from a recorded off-axis hologram is carried out in analogy to reconstruction from an in-line hologram. The first-order beams then propagate including an angle $\pm \theta_{\text {eff }}$ with the optical axis. Alternatively, reconstruction using a reference beam with an incidence angle $\theta_{\text {eff }}$ yields a first-oder beam propagating along the optical axis, which is more practical for analysing its phase (see Section A.4.4).

\section{A.3 Equivalence of the paraxial approximation of the Fresnel-Kirchhoff integral and the parabolic wave equation in free space}

According to Eq. (5.7) and Eq. (5.8), the propagation of the electric field $E$ over a distance $z$ from a plane with a known field $E_{1}$ is described in Fourier space by

$$
\tilde{E}=\tilde{h}_{z} \times \tilde{E}_{1}
$$

with

$$
\tilde{h}_{z}\left(\nu_{x}, \nu_{y}\right)=-\exp (i k z) \times \exp \left[-i \pi \lambda z\left(\nu_{x}{ }^{2}+\nu_{y}{ }^{2}\right)\right] .
$$

In analogy to Eq. (2.44) we may define $u(x, y, z)$ by

$$
E(x, y, z)=u(x, y, z) \exp (i k z) .
$$

Correspondingly, we find in Fourier space

$$
\tilde{u}=\exp \left[-i \pi \lambda z\left(\nu_{x}^{2}+\nu_{y}^{2}\right)\right] \tilde{E}_{1} .
$$

The partial derivative $\partial \tilde{u} / \partial z$ is thus given by

$$
\begin{aligned}
\frac{\partial \tilde{u}}{\partial z} & =\frac{\partial}{\partial z}\left(\exp \left[-i \pi \lambda z\left(\nu_{x}^{2}+\nu_{y}^{2}\right)\right]\right) \tilde{E}_{1} \\
& =-i \pi \lambda\left(\nu_{x}^{2}+\nu_{y}^{2}\right) \exp \left[-i \pi \lambda z\left(\nu_{x}^{2}+\nu_{y}^{2}\right)\right] \tilde{E}_{1} \\
& =-i \pi \lambda\left(\nu_{x}^{2}+\nu_{y}^{2}\right) \tilde{u}
\end{aligned}
$$

Application of Eq. A.3 then yields

$$
\frac{\partial u}{\partial z}=\frac{-i \pi \lambda}{(-2 \pi i)^{2}}\left(\frac{\partial^{2}}{\partial x^{2}}+\frac{\partial^{2}}{\partial y^{2}}\right) u=\frac{i}{2 k}\left(\frac{\partial^{2}}{\partial x^{2}}+\frac{\partial^{2}}{\partial y^{2}}\right) u .
$$

This may also be written as

$$
2 i k \frac{\partial u}{\partial z}+\left(\frac{\partial^{2}}{\partial x^{2}}+\frac{\partial^{2}}{\partial y^{2}}\right) u=0
$$

which is the parabolic wave equation in the coordinate system used in the imaging chapters using the ansatz A.32 and $n=1$. 


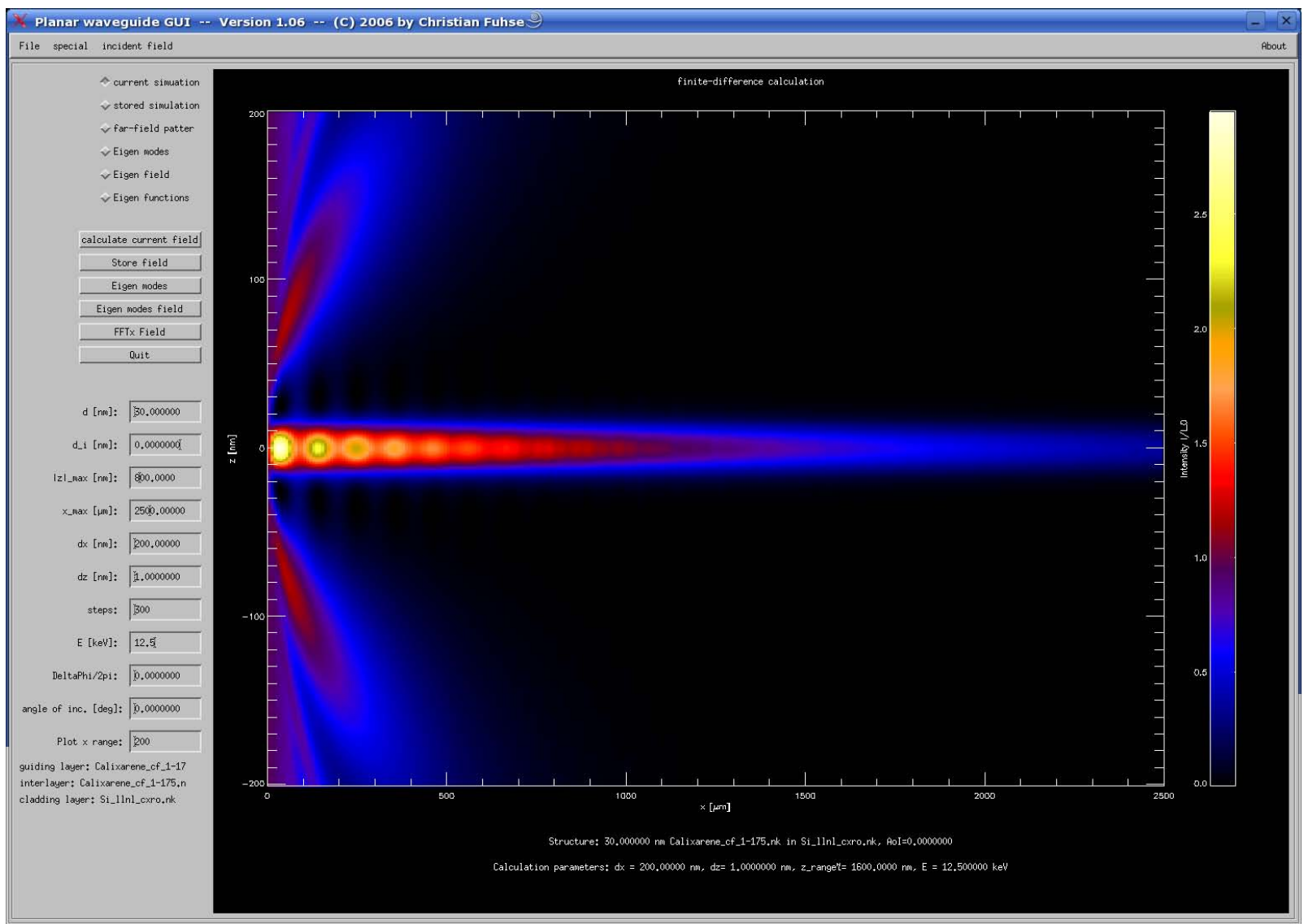

Figure A.1: The finite-difference scheme to calculate the electric field in one-dimensionally confining waveguides was implemented in IDL. The program provides a graphical user interface for slab waveguides.

\section{A.4 IDL source code}

This section presents the essential procedures of the computer programs developed within the scope of this thesis. The programs are written in the IDL programming language* Version 6.0.

\section{A.4.1 Finite-difference calculations in two dimensions}

The finite-difference algorithm presented in Section 3.2 to solve the parabolic wave equation in two dimensions was implemented in IDL. A program with a graphical user interface was written for field calculations of one-dimensionally confined waveguides (Fig. A.1). Since the complete code is too long for this thesis, only the procedure carrying out the finitedifference algorithm is given here. In IDL, comments are denoted by a colon and a dollar sign indicates that the respective command is split over multiple lines.

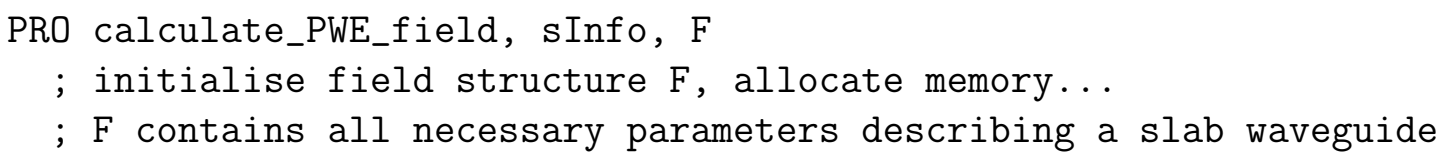

*IDL is a registered trademark of Research Systems Inc., http://www.rsinc.com 


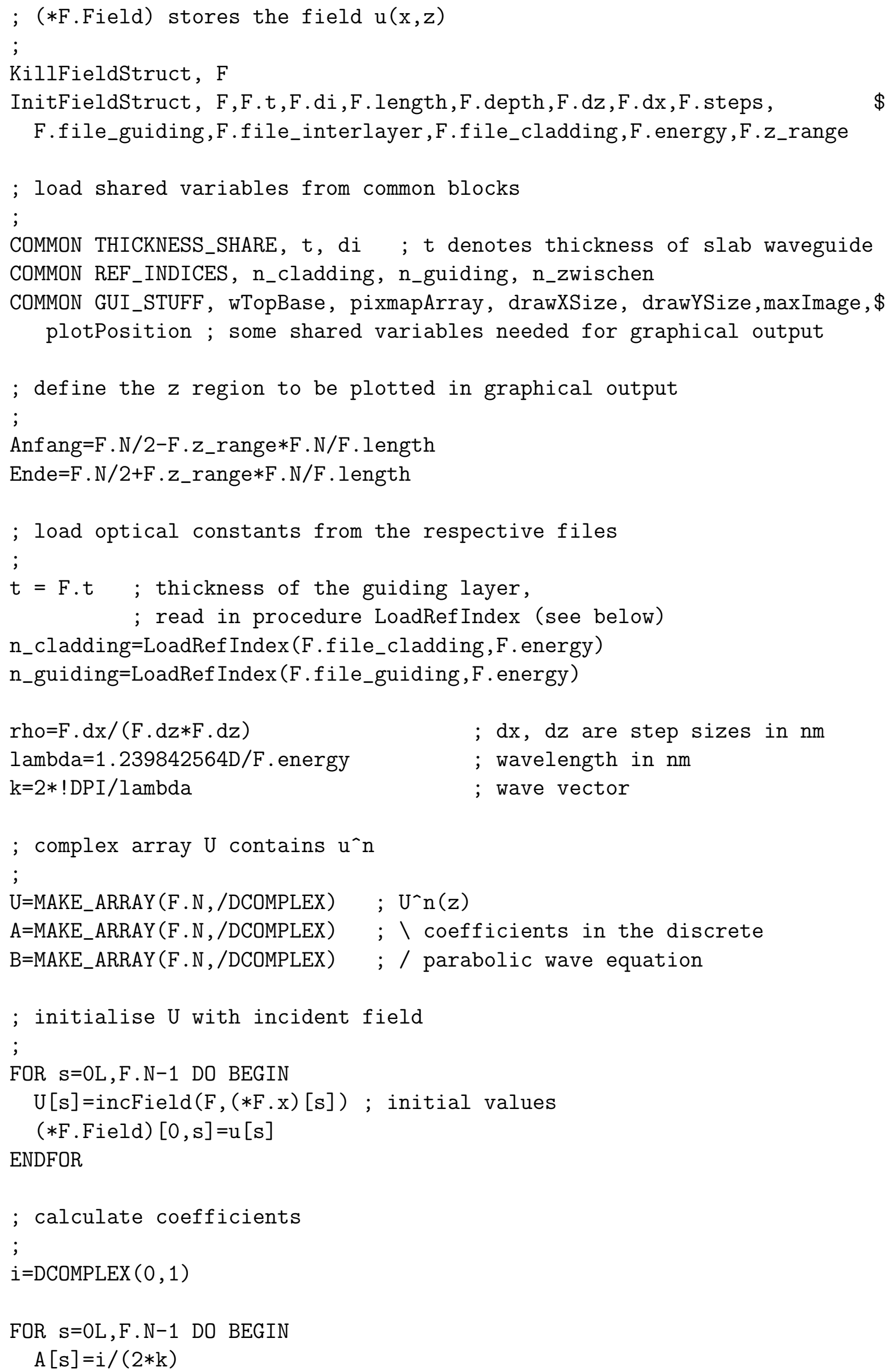




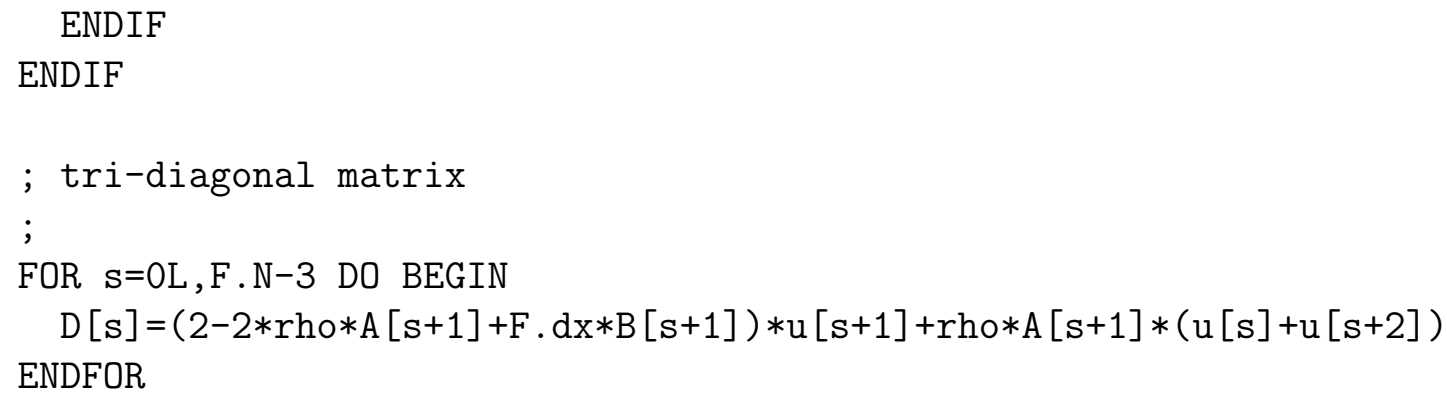

\section{A.4.2 Finite-difference calculations in three dimensions}

The finite-difference algorithm presented in Section 3.3 to solve the parabolic wave equation in three dimensions was implemented in IDL. Fig. A.2 shows the graphical output window of the program. Since the complete code is too long for this thesis, again only the procedure carrying out the finite-difference algorithm is presented.

PRO tdPWEsolve, info

; get shared variables from COMMON blocks

;

COMMON WG, tdWaveguide

COMMON RefInd_n, n_cladding, $\mathrm{n}$ _guiding 


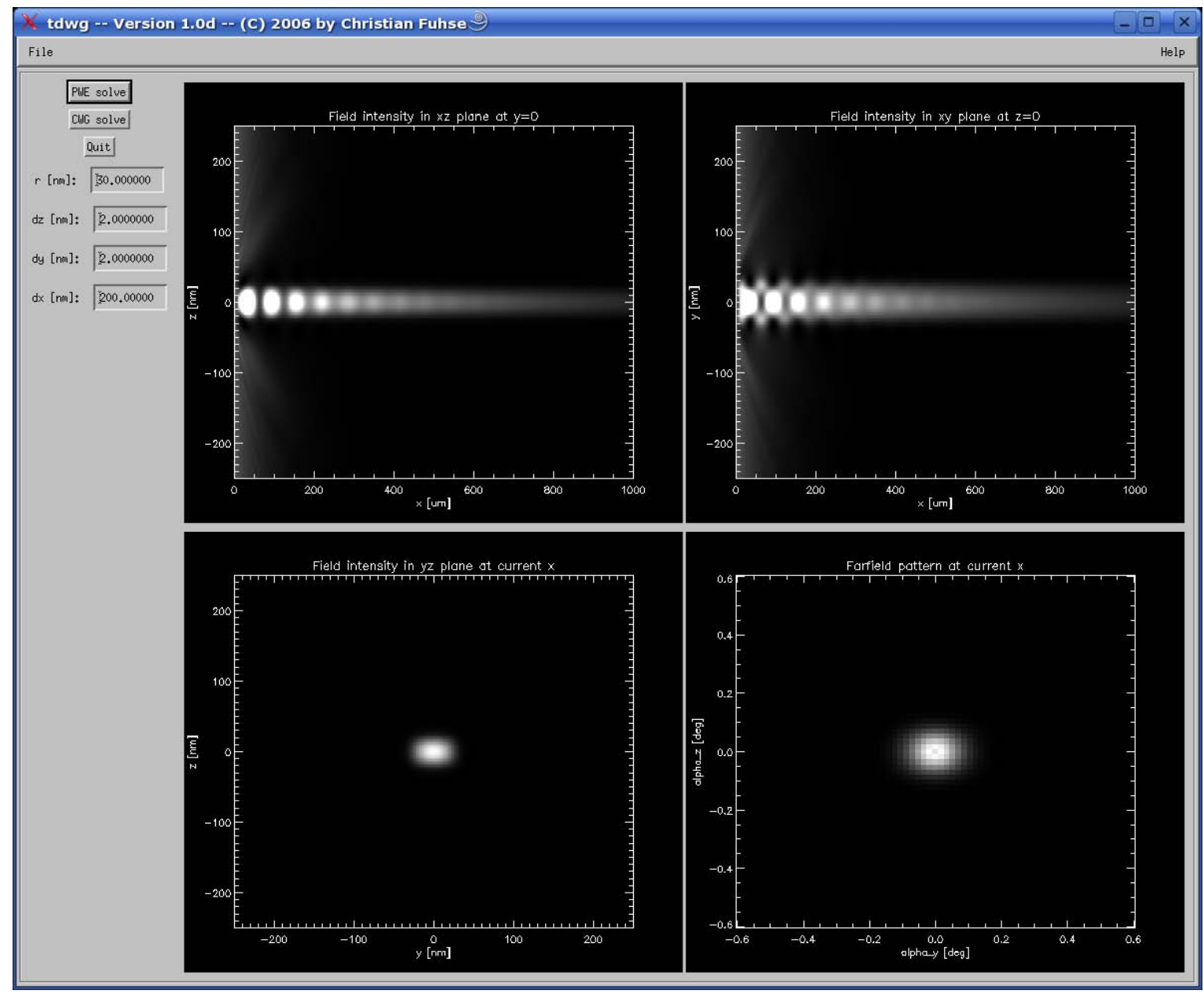

Figure A.2: The finite-difference scheme to calculate the electric field in two-dimensionally confining waveguides was implemented in IDL. The program provides a graphical output. 


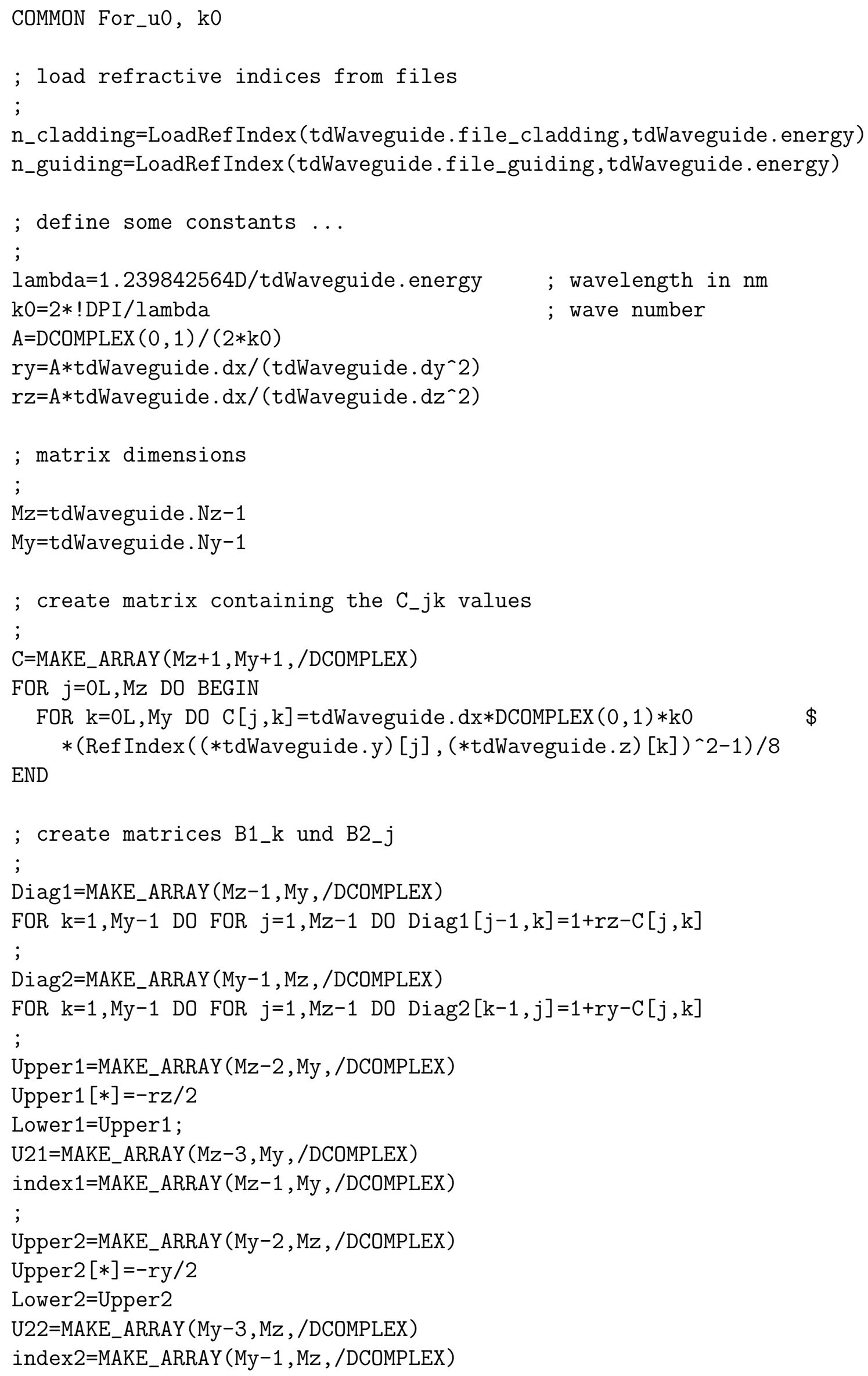




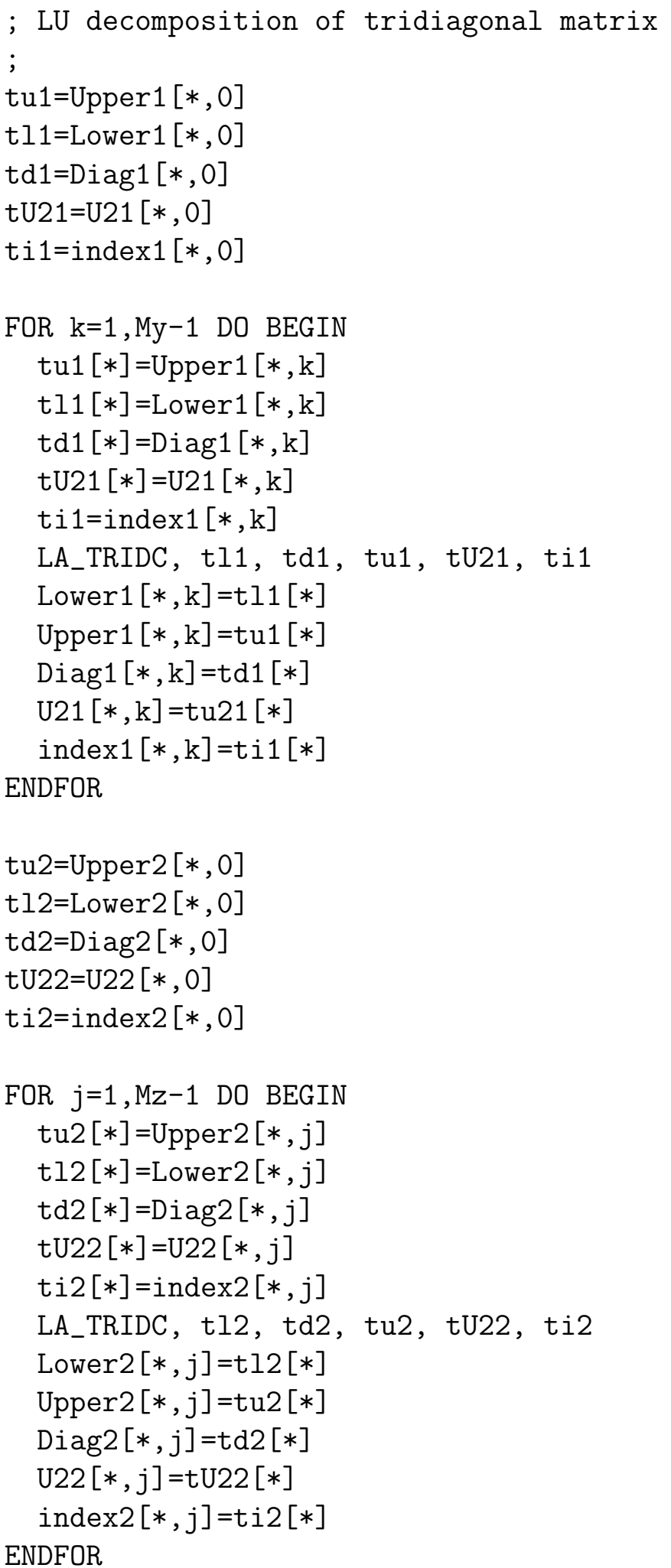

; initialise $\mathrm{u}$ with incident wave ;

FOR $i=0$, Mz DO FOR $j=0$, My DO $\$$

(*tdWaveguide.u) $[i, j]=u 0(0,(*$ tdWaveguide.y) $[i],(*$ tdWaveguide.z) $[j])$

unew $=*$ tdWaveguide.$u$ ; array for storing $u_{-} k^{\wedge}(n+1 / 2)$ 


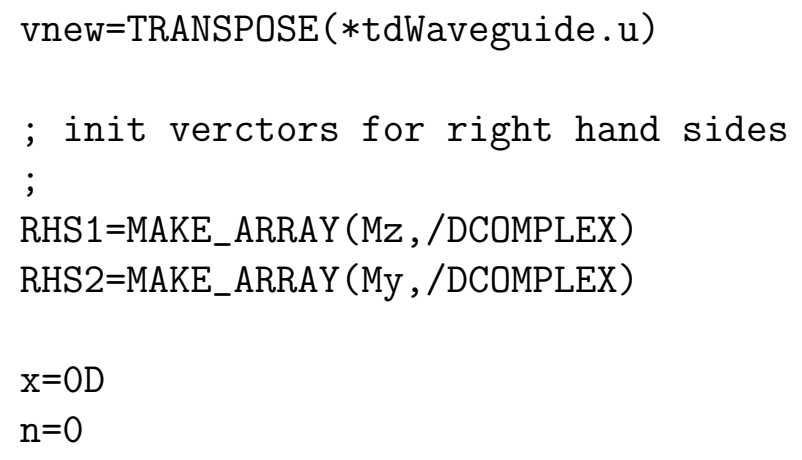


$\mathrm{x}+=$ tdWaveguide $. \mathrm{dx} / 2$

FOR $j=0, M z$ DO BEGIN

vnew $[0, j]=\mathrm{u} 0(\mathrm{x},(*$ tdWaveguide. $\mathrm{y})[j],(*$ tdWaveguide. $z)[0])$

vnew $[$ My,$j]=u 0(x,(*$ tdWaveguide.y) $[j],(*$ tdWaveguide. $z)[M y])$

END

FOR $\mathrm{k}=0$, My DO BEGIN

vnew $[\mathrm{k}, 0]=\mathrm{u} 0(\mathrm{x},(*$ tdWaveguide $\cdot \mathrm{y})[0],(*$ tdWaveguide. $\mathrm{z})[\mathrm{k}])$

vnew $[\mathrm{k}, \mathrm{Mz}]=\mathrm{u} 0(\mathrm{x},(*$ tdWaveguide.y) $[\mathrm{Mz}],(*$ tdWaveguide. $\mathrm{z})[\mathrm{k}])$

END

; second half step of Peaceman-Rachford scheme $\ldots$; ;

FOR $j=1, M z-1$ DO BEGIN

; init right hand side

FOR $k=1$, My-1 DO RHS2 $[k]=(1-r z+C[j, k]) * u t[k, j]+r z / 2 *(u t[k, j-1]+u t[k, j+1])$

RHS2 [1] +=ry $/ 2 *$ vnew $[0, j]$

RHS2 [My-1]+=ry/2*vnew [My,$j]$

; solve tridiagonal matrix equation

vnew $[1: \operatorname{My}-1, j]=$ LA_TRISOL (Lower $2[*, j], \operatorname{Diag} 2[*, j], \operatorname{Upper} 2[*, j], \$$

ENDFOR

$\mathrm{U} 22[*, j]$, index $[*, j], \operatorname{RHS} 2[1: \mathrm{My}-1])$

*tdWaveguide. $u=$ TRANSPOSE (vnew)

pos $=\operatorname{MIN}\left(\left[\left(\mathrm{x} *(\right.\right.\right.$ tdWaveguide $. \mathrm{Nx}-1) /$ tdWaveguide. $\left.\mathrm{x} \_\max \right)$, tdWaveguide $\left.\left.. \mathrm{Nx}-1\right]\right)$

(*tdWaveguide.FieldProj_xz) $[0: \mathrm{Mz}, \mathrm{pos}]=(*$ tdWaveguide.u) $[*, \mathrm{my} / 2]$

(*tdWaveguide.FieldProj_xy) $[0:$ My,pos] $=(*$ tdWaveguide.u) $[\mathrm{mz} / 2, *]$

$\mathrm{n}++$

ENDWHILE

END; of tdPWEsolve

\section{A.4.3 A propagation algorithm for holographic reconstruction}

The following procedure propagates a field stored in the two-dimensional matrix Holo over a distance $\mathbf{z}$ and stores the result in the matrix Reconstruction. Based on Eq. (5.7), the Fresnel-Kirchhoff integral is calcualted within the paraxial approximation using a discrete Fourier transformation. Therefore, the fast Fourier transfomation (FFT) procedure provided by IDL 6.0 is applied.

PRO propagateFFT

; COMMON blocks contain variables shared with other procedure COMMON Dimensions, $\mathrm{Nx}, \mathrm{Ny}, \mathrm{D}_{-} \mathrm{x}, \mathrm{D}_{-} \mathrm{y}$; size of the matrices below

; and corresponding lengths

COMMON Fields, Holo, Reconstruction ; Nx * Ny matrices of type

; DCOMPLEX

COMMON Coordinates, $\mathrm{x}, \mathrm{y}, \mathrm{z}$; corresponding coordinates, i. e.

; Holo $[i, j]$ is the field at (x[i],y[j])

COMMON Wavelength, lambda, $\mathrm{k}$; wavelength and wave number $\mathrm{I}=\operatorname{DCOMPLEX}(0,1)$

; imaginary unity 


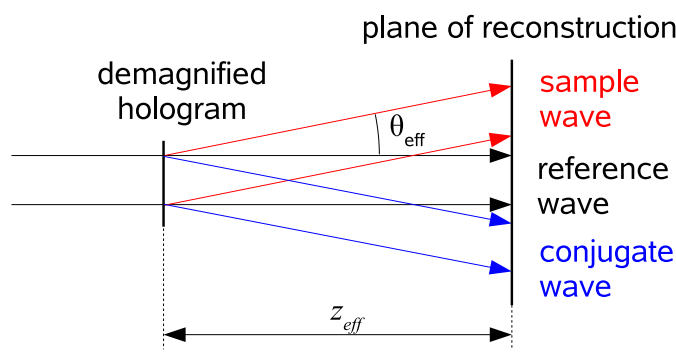

(a)

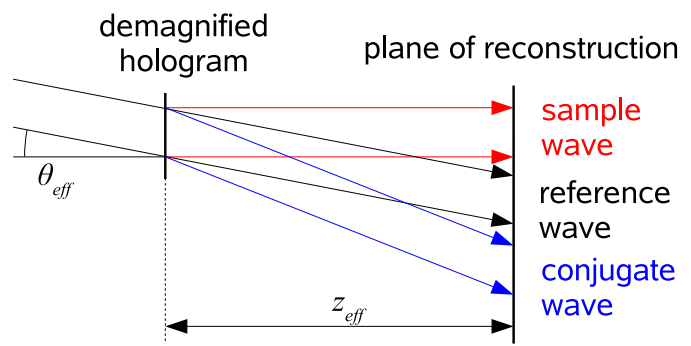

(b)

Figure A.3: Numerical reconstruction from an off-axis hologram. (a) When the reference wave hits the hologram at normal incidence, the first-order beam propagates including an angle $\theta_{\text {eff }}$ with the optical axis. Accordingly, phase of the first-order beam rapidly oscillates in the plane of reconstruction. (b) Using a reference wave incident at an angle $\theta_{\text {eff, }}$ the first-order beam containing the focused image propagates in the direction of the optical axis. Phase in the plane of reconstruction then directly corresponds to the phase of the optical transmission function of the sample.

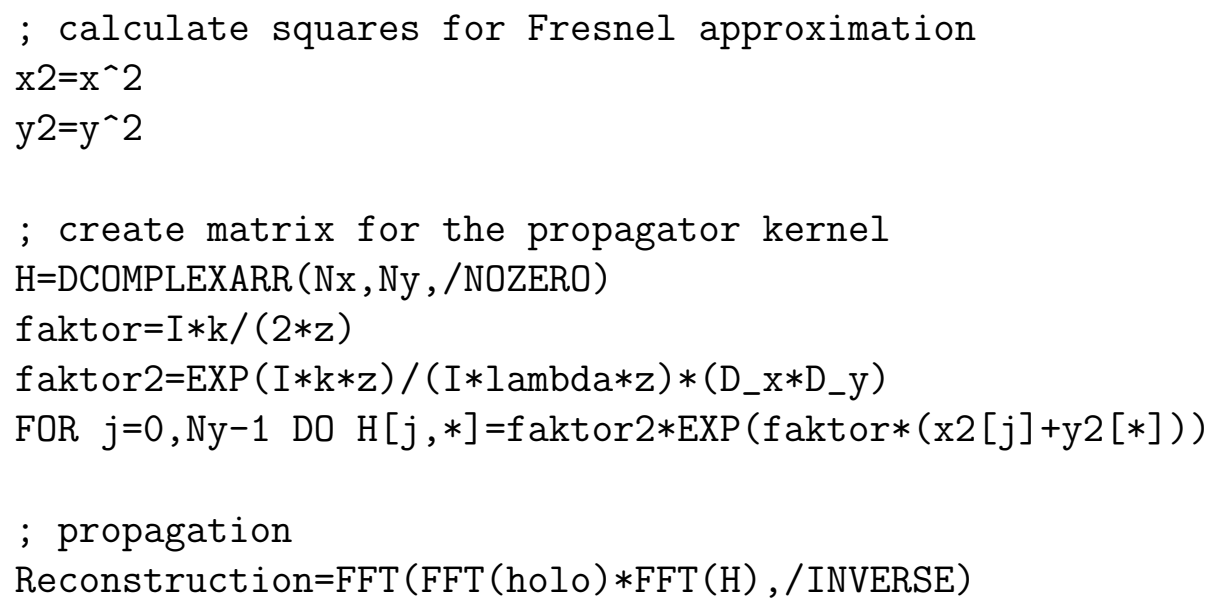

\section{A.4.4 Off-axis holographic reconstruction}

In the following, a complete IDL program is given which carries out holographic reconstruction from an off-axis hologram. The main procedure is wgholo and reconstruction is carried out as illustrated in Fig. A.3(b), The reference beam hits the hologram at an angle $\theta_{\text {eff }}$ to ensure that the first-order beam containing the focused image is propagating along the optical axis. Apart from a constant offset, phase in the plane of reconstruction then directly corresponds to the phase of the optical transmission function related to the sample. The hologram is read in from a TIFF file and the result is displayed on the screen (Fig. A.4).

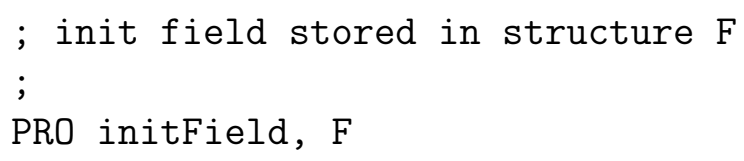



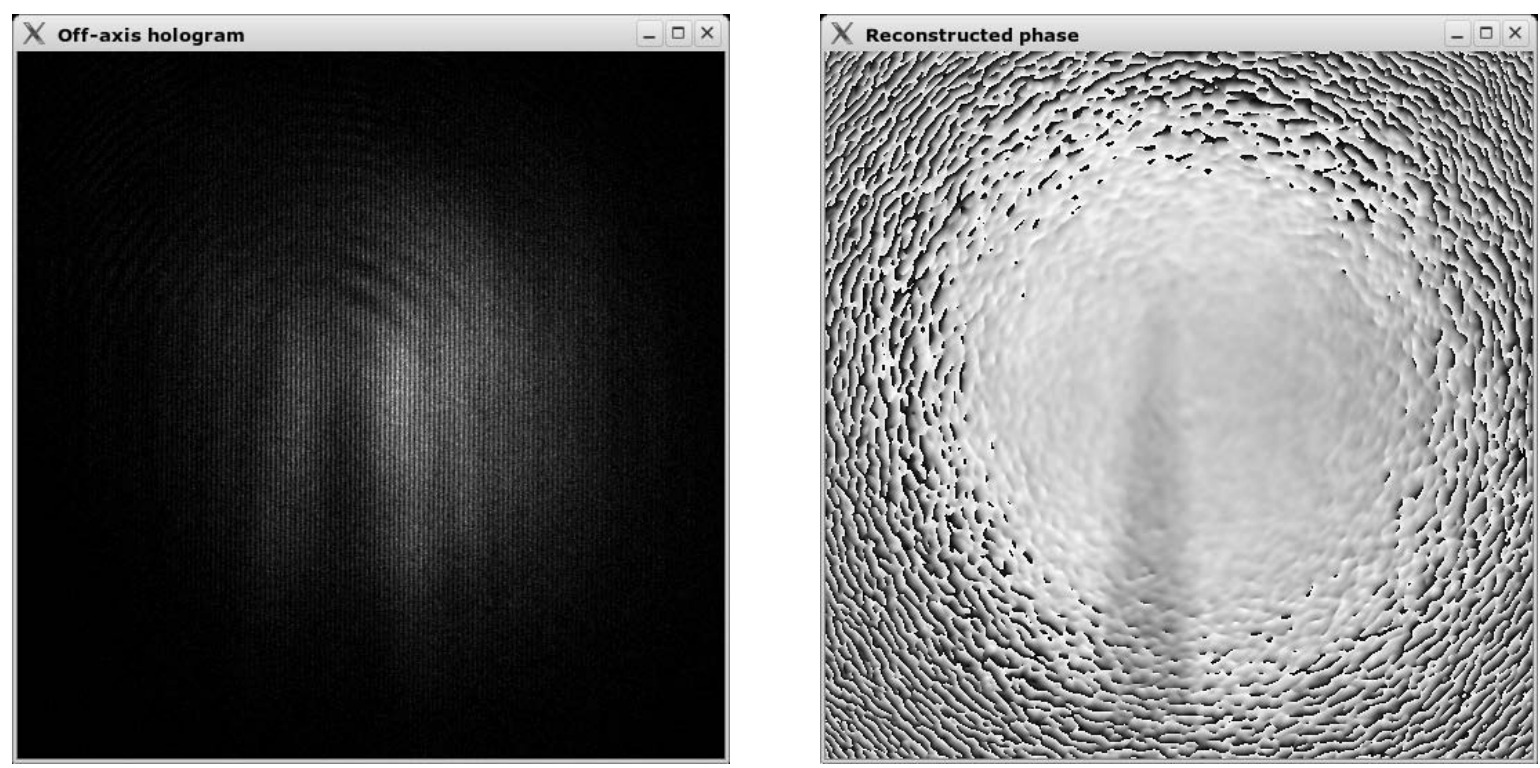

Figure A.4: Graphical output of the IDL program for off-axis reconstruction showing the recorded hologram (left) and phase of the reconstruction (right).

END ; of initField

F . A $=$ PTR_NEW (MAKE_ARRAY ( F . Nx , F.Ny , /DCOMPLEX , VALUE $=1))$

$\mathrm{F} . \mathrm{X}=\mathrm{PTR} \_\mathrm{NEW}\left((\mathrm{DINDGEN}(\mathrm{F} . \mathrm{Nx})-(\mathrm{F} . \mathrm{Nx}-1) / 2.0) /(\mathrm{F} \cdot \mathrm{Nx}-1.0) * \mathrm{~F} \cdot \mathrm{D}_{-} \mathrm{x}\right)$

$; \mathrm{x}=\left(0, \ldots, \mathrm{D}_{-} \mathrm{x} / 2,-\mathrm{D}_{-} \mathrm{x} / 2, \ldots,-\mathrm{D}_{-} \mathrm{x} / \mathrm{Nx}\right)$

$\mathrm{F} \cdot \mathrm{y}=\mathrm{PTR} \_$NEW $\left((\mathrm{DINDGEN}(\mathrm{F} . \mathrm{Ny})-(\mathrm{F} . \mathrm{Ny}-1) / 2.0) /(\mathrm{F} . \mathrm{Ny}-1.0) * \mathrm{~F} . \mathrm{D} \_\mathrm{y}\right)$

$; \mathrm{y}=\operatorname{SHIFT}(* \mathrm{~F} \cdot \mathrm{y}, \mathrm{F} \cdot \mathrm{Ny} / 2)-\mathrm{F} . \mathrm{D}_{-} \mathrm{y} /(2 * \mathrm{~F} . \mathrm{Ny})$

; F : field to be propagated

;

FUNCTION propagate_field_FFT, F,z

COMMON Wavelength, lambda, k, Energy

$\mathrm{x}=\operatorname{SHIFT}(* \mathrm{~F} \cdot \mathrm{x}, \mathrm{F} \cdot \mathrm{Nx} / 2)-\mathrm{F} \cdot \mathrm{D} \_\mathrm{x} /(2 * \mathrm{~F} \cdot \mathrm{Nx})$

$; \mathrm{x}=\left(0, \ldots, \mathrm{D}_{-} \mathrm{x} / 2,-\mathrm{D}_{-} \mathrm{x} / 2, \ldots,-\mathrm{D}_{-} \mathrm{x} / \mathrm{Nx}\right)$

$\mathrm{y}=\operatorname{SHIFT}(* \mathrm{~F} \cdot \mathrm{y}, \mathrm{F} \cdot \mathrm{Ny} / 2)-\mathrm{F} . \mathrm{D}_{-} \mathrm{y} /(2 * \mathrm{~F} \cdot \mathrm{Ny})$

$; \mathrm{y}=\left(0, \ldots, D_{-} \mathrm{y} / 2,-\mathrm{D}_{-} \mathrm{y} / 2, \ldots,-\mathrm{D}_{-} \mathrm{y} / \mathrm{Ny}\right)$

I=DCOMPLEX $(0,1)$; imaginary unity

; create matrix containing the propagator kernel

;

$\mathrm{H}=\mathrm{DCOMPLEXARR}(\mathrm{F} . \mathrm{Nx}, \mathrm{F} . \mathrm{Ny}, /$ NOZERO $)$

faktor $=\mathrm{I} * \mathrm{k} /(2 * \mathrm{z})$

faktor $2=\mathrm{EXP}(\mathrm{I} * \mathrm{k} * \mathrm{z}) /(\mathrm{I} * \mathrm{lambda} * \mathrm{z}) *\left(\mathrm{~F} . \mathrm{D} \_\mathrm{X} * \mathrm{~F}\right.$.D_y $)$

FOR $\mathrm{j}=0, \mathrm{~F} \cdot \mathrm{Nx}-1$ DO $\$$

$$
\mathrm{H}[\mathrm{j}, *]=\text { faktor } 2 * \operatorname{EXP}\left(\text { faktor } *\left(x[j]^{\wedge} 2+y[*]^{\wedge} 2\right)\right)
$$

; propagate in reciprocal space 
;

$\mathrm{H}=\mathrm{FFT}(\mathrm{H})$

; Fourier backtransform return result

;

RETURN , FFT (FFT ( $*$ F . A) $*$ H , / INVERSE)

END; of function propagate_field_FFT

; off-axis holographic reconstruction

;

PRO wgholo

COMMON Wavelength, lambda, k, Energy ;

$\mathrm{I}=\operatorname{DCOMPLEX}(0,1) \quad$; imaginary unity

; lambda $=1 \mathrm{e}-10 \mathrm{~d}$; all lengths measured in meters

Energy=10.4 ; photon enrgy [keV]

lambda $=1.239842564 \mathrm{D} /$ Energy*1e-9 ; wavelength [m]

$\mathrm{k}=2 *$ ! DPI/lambda ; wave number $[1 / \mathrm{m}]$

eff_defoc_dist=0.00125 ; eff. defoc. distance [m]

; init structure Field containing the electric field

;

Field $=\{$ FieldStruct,

$\mathrm{Nx}: 2048, \quad \$$; pixels per row

Ny : 1024, \$; pixels per column

D_x : $17.36 \mathrm{e}-6, \$$; dimensions in meters

D_y : $8.54 \mathrm{e}-6, \quad \$ ;$

A : PTR_NEW(), $\$$; pointer to field (DCOMPLEX array)

$\mathrm{x}$ : PTR_NEW( $), \quad \$ ; \mathrm{x}$ values

$\mathrm{y}:$ PTR_NEW() $\$$; y values

\}

initField, Field

; store coordinated in matrices

;

$\mathrm{yc}=*(\mathrm{Field} \cdot \mathrm{y})$ \#\#MAKE_ARRAY (Field.Nx, /DOUBLE, VALUE=1)

$\mathrm{xc}=$ MAKE_ARRAY $($ Field. Ny,$/$ DOUBLE, VALUE $=1) \# \#((*$ Field.$x))$

; read recorded hologram from file

;

rholo=READ_TIFF('Ge0520_97_32.tif')

WINDOW , 0, XSIZE=512, YSIZE=512, TITLE="Off-axis hologram" , RETAIN=2

TV, CONGRID (rholo, 512, 512)

; store hologram in matrix FresnelInt

; 
*Field. A=DCOMPLEXARR (Field. Nx, Field. Ny)

FOR $j=0,511$ DO (*Field.A) $[768: 768+511,256+j]=\operatorname{rholo}[0: 511, j]$

; wave vector components of the reference wave

; ( the ky component may correct a small tilt between the

$; \mathrm{x}$ axis of the detector and the double waveguide)

;

$\mathrm{kx}=-572.12 * 2 *$ !DPI/Field.D_x $*$ Field.Nx/(Field.NX+1)

$\mathrm{ky}=-1.5 * 2 *$ ! DPI/Field.D_y

; illuminate hologram with plane reference wave

$*$ Field.A*=EXP $(\operatorname{DCOMPLEX}(0,1) *(\mathrm{xc} * \mathrm{kx}+\mathrm{yc} * \mathrm{ky}))$

; propagate...

Reconstruction=propagate_field_FFT(Field,-eff_defoc_dist) $* \$$ $\operatorname{DCOMPLEX}(-1,0)$;

; graphical output of reconstructed phase

phase $=$ BYTSCL $($ ATAN (Reconstruction $[768: 768+512,256: 256+511], /$ PHASE) $), \$$ $M I N=-! D P I, M A X=$ ! DPI)

WINDOW, 1, XSIZE=512, YSIZE=512, TITLE="Reconstructed phase" , RETAIN=2 TV, phase

END 


\section{B List of publications}

- H. U. Krebs, M. Weisheit, J. Faupel, E. Sueske, T. Scharf, C. Fuhse, M. Stoermer, K. Sturm, M. Seibt, H. Kijewski, D. Nelke, E. Panchenko, and M. Buback, Pulsed laser deposition (PLD) - a versatile thin film technique, Adv. Sol. State Phys. 43, 505-518 (2003).

- C. Fuhse, H.-U. Krebs, S. Vitta, and G. A. Johansson, Interface quality and thermal stability of laser-deposited metal/MgO multilayers, Appl. Opt. 43, 6265-6269 (2004).

- J. Faupel, C. Fuhse, A. Meschede, C. Herweg, H.-U. Krebs, and S. Vitta, Microstructure of pulsed laser deposited ceramic-metal and polymer-metal nanocomposite thin films,

Appl. Phys. A 79, 1233-1235 (2004).

- C. Fuhse, A. Jarre, C. Ollinger, J. Seeger, T. Salditt, and R. Tucoulou, Front-coupling of a pre-focused x-ray beam into a mono-modal planar waveguide, Appl. Phys. Lett. 85, 1907-1909 (2004).

- C. Ollinger, C. Fuhse, A. Jarre, and T. Salditt, Two-dimensional $X$-ray waveguides on a grating,

Physica B, 357, 53-56 (2005).

- C. Fuhse and T. Salditt, Finite-difference field calculations for one-dimensionally confined X-ray waveguides, Physica B, 357, 57-60 (2005).

- A. Jarre, C. Fuhse, C. Ollinger, J. Seeger, R. Tucoulou, and T. Salditt Two-Dimensional Hard X-Ray Beam Compression by Combined Focusing and Waveguide Optics, Phys. Rev. Lett. 94, 074801 (2005).

- C. Fuhse, C. Ollinger, S. Kalbfleisch, and T. Salditt, Coherent propagation of white X-rays in a planar waveguide, J. Synchrotron Rad. 13, 69-73 (2006).

- C. Fuhse and T. Salditt, Propagation of x-rays in ultra-narrow slits, Opt. Commun., in press. 
- C. Fuhse and T. Salditt,

Finite-Difference Field Calculations for Two-Dimensionally Confined X-ray Waveguides,

Appl. Opt., in press. 


\section{Acknowledgements}

I would like thank Tim Salditt for proposing this interesting topic and for outstanding interest and continuous support for this work. I am additionally grateful to Hans-Ulrich Krebs, who is going to co-referee this thesis.

It was a great pleasure to work together with Christoph Ollinger, Ansgar Jarre, Jens Seeger and Sebastian Kalbfleisch: the "waveguide team". I am eminently grateful to Christoph Ollinger, from whose experimental skills and in particular the "Mobile and modular waveguide stage" this work has profited enormously. I am furthermore pleased that Anika Hillmann and Sebastian Panknin are going to continue our work on X-ray waveguides or, more general, X-ray optical elements.

Going beyond the "waveguide team", I really enjoyed working at the Institut für Röntgenphysik and I would like to thank everybody there for the friendly atmosphere. Regarding this work, I would like to thank Jochen Herbst, in particular for an introduction to the electron beam evaporation system and carrying out many of the depositions. I am furthermore grateful to Thorsten Gronemann who has, among other things, prepared the thin Si foils. I thank Guillaume Brotons for many interesting discussions and Doru Constantin for sharing his profound mathematical knowledge.

The present experiments unconditionally required the use of synchrotron radiation and I acknowledge provision of beamtime and travel reimbursement by the European Synchrotron Radiation Facility (ESRF, Grenoble), the Deutsche Elektronensynchrotron (DESY, Hamburg), and the Berliner Elektronenspeicherring (BESSY II, Berlin).

Regarding individual beamtimes, I would like to thank Remi Tucoulou for excellent experimental conditions at ID22 (ESRF). I thank Norbert Schell for beamtime and technical assistance at the Rossendorf beamline (ROBL, ESRF). I thank Ullrich Pietsch for providing beamtime at the EDR beamline (BESSY II) as well as Wolfram Leiterberger and Yves Bodenthin for corresponding experimental support. I would like to thank Anders Madsen and Andrei Fluerasu for beamtime and technical assistance at ID10A (ESRF). Finally, I acknowledge several beamtimes at D4.1 (DESY).

Furthermore, I acknowledge the contributions of several members of the Institut für Materialphysik. I would like to thank Thorsten Scharf and Johanna Röder for preparing laser deposited test samples for the imaging experiments, I thank Talaát Al-Kassab for providing the tungsten tips, and I thank Peter-Joachim Wilbrandt for polishing the end faces of the waveguides used for imaging experiments by focused ion beam.

I thank Hauke Schollmeyer, Jürgen Thieme and Sebastian Panknin for proof-reading the manuscript, or parts of it, and for many helpful suggestions. Daniel Ross, as a native speaker, has carefully read the manuscript. 
This work was supported by the German Federal Ministry of Education and Research (BMBF) under grant no. $05 \mathrm{KS} 4 \mathrm{MGA} / 9$.

Nicht zuletzt danke ich meiner Familie für ihre stete Unterstützung, ohne die mein Studium und diese Arbeit nicht möglich gewesen wären. 


\section{Lebenslauf}

Christian Fuhse

geboren am 8. April 1977 in Osterode am Harz,

Staatsangehörigkeit: deutsch

\section{Schulbildung}

1983-1987

1987-1989

1989-1996

\section{Wehrdienst}

1996-1997

\section{Studium}

$1997-2006$

2002

seit 2003
Grundschule Gittelde

Orientierungsstufe Badenhausen

Gymnasium Osterode am Harz,

abgeschlossen mit der Allgemeinen Hochschulreife

Grundwehrdienst bei der Luftwaffe in Oldenburg (Oldb.)
Physik-Studium an der Georg-August-Universität Göttingen Diplom in Physik, Titel der Arbeit: "Struktur und Grenzflächenrauigkeiten laserdeponierter Metall/Keramik-Schichtsysteme", Institut für Materialphysik, Georg-August-Universität Göttingen wissenschaftlicher Mitarbeiter am Institut für Röntgenphysik, Georg-August-Universität Göttingen 